\title{
CYCLIC DEFORMATION BEHAVIOR OF RARE-EARTH CONTAINING MAGNESIUM ALLOYS
}

\author{
by \\ MIRZA FOISAL AHMED \\ Master of Engineering in Mechanical Engineering \\ Changwon National University, Changwon, South Korea, 2010 \\ Bachelor of Science in Mechanical Engineering \\ Bangladesh University of Engineering and Technology, Bangladesh (BUET), 2006
}

A dissertation
presented to Ryerson University

in partial fulfillment of the

requirements for the degree of

Doctor of Philosophy

in the Program of

Mechanical and Industrial Engineering

Toronto, Ontario, Canada, 2014

(C) Mirza Foisal Ahmed 2014 


\section{AUTHOR'S DECLARATION}

I hereby declare that I am the sole author of this dissertation. This is a true copy of the dissertation, including any required final revisions, as accepted by my examiners.

I authorize Ryerson University to lend this dissertation to other institutions or individuals for the purpose of scholarly research.

I further authorize Ryerson University to reproduce this dissertation by photocopying or by other means, in total or in part, at the request of other institutions or individuals for the purpose of scholarly research.

I understand that my dissertation may be made electronically available to the public. 


\title{
CYCLIC DEFORMATION BEHAVIOR OF RARE-EARTH CONTAINING MAGNESIUM ALLOYS
}

\author{
(C) Mirza Foisal Ahmed, 2014 \\ Doctor of Philosophy \\ Department of Mechanical \& Industrial Engineering \\ Ryerson University
}

\begin{abstract}
Lightweighting in ground vehicles is considered as one of the most effective strategies to improve fuel economy and reduce anthropogenic environment-damaging, climate-changing and costly emissions. Magnesium (Mg) alloy, as a strategic ultra-lightweight metallic material, has recently drawn a considerable interest in the transportation industry to reduce the weight of vehicles due to their high strength-to-weight ratio, dimensional stability, and good machinability and recyclability. However, the hexagonal close-packed crystal (HCP) structure of Mg alloys gives only limited slip systems and develops sharp crystallographic textures associated with strong mechanical anisotropy and tension-compression yield asymmetry. For the vehicle components subjected to dynamic loading, such asymmetry could exert an unfavorable influence on the material performance. These problems could be tackled through texture modification via addition of rare-earth (RE) elements. These RE-Mg alloys possess relatively weak initial textures, which lead to improved ductility and strength, and a reduction of the tension-compression
\end{abstract}


asymmetry present in the conventional wrought $\mathrm{Mg}$ alloys. Despite the fact that the addition of RE elements sheds some light on the alterations in the mechanical anisotropy and the tensioncompression yield asymmetry, the potential advantage of such RE-Mg alloys as structural components under cyclic loading condition has not been well appreciated. Thus, the main objective of this dissertation was to explore the cyclic deformation behavior of RE-Mg alloys under varying strain amplitudes and strain ratios, and correlate the behavior to the microstructural change and crystallographic texture weakening in the RE-Mg alloys in different states (extruded and heat-treated). Unlike the RE-free $\mathrm{Mg}$ alloys, these alloys exhibited essentially cyclic stabilization and fairly symmetrical hysteresis loops due to the weaker texture and reduced twinning-detwinning activities. While these alloys had a lower cyclic strain hardening exponent than the RE-free extruded $\mathrm{Mg}$ alloys, it had a longer fatigue life which can also be described by the Coffin-Manson law and Basquin's equation. Fatigue crack was observed to initiate from the specimen surface with some cleavage-like facets near the initiation site. Crack propagation was basically characterized by fatigue striations in conjunction with secondary cracks. A detailed analysis for understanding the obstructive role of the precipitate to twinning has been also presented. 


\section{ACKNOWLEDGEMENTS}

Foremost, I wish to express my deepest gratitude to my supervisor Dr. Daolun Chen, for his invaluable guidance, support, and generosity during my studies at Ryerson University and for providing me with every opportunity and believing me in all aspects of my studies. I was fortunate to have him as my advisor and it was my pleasure to work with a man who has broad and profound knowledge, not only in the academic field, but also in many other aspects. I highly appreciate his scientific rigorousness and encouragement from different perspectives for my academic research and future professional life. I would also like to sincerely thank Professor S.D. Bhole for his helpful suggestions from time to time.

I would like to thank the Natural Sciences and Engineering Research Council of Canada (NSERC) and AUTO21 for providing financial support. This investigation involves part of the Canada-China-USA Collaborative Research Project on the Magnesium Front End Research and Development (MFERD). I would also like to thank Prof. X.Q. Zeng from Shanghai Jiao Tong University, Shanghai, PR China for the supply of rare-earth containing magnesium alloys and

Prof. A.A. Luo from Ohio State University (formerly with General Motors Research and Development Center) for the supply of extruded AM30 magnesium alloy.

I would like to extend my thanks to all my friends and colleagues at Ryerson University for helping me and keeping my spirits up. Special thanks are owed to Messrs. A. Machin, Q. Li, J. Amankrah, C. Ma, G. He, and R. Churaman for easy access to the laboratory facilities of Ryerson University and their assistance in the experiments. 
Finally, while I offer everything I do in life up to Almighty Allah, I dedicate this particular piece of work to my mother Mrs. Abida Sultana who is the rock upon which all of my current and future success has been and will be built. She provided me with love, encouragement and support, without which this wouldn't be possible for me. Moreover, words cannot express my deepest gratitude towards my siblings: Maymuna Sultana, Mahfuz Chowdhury, Kari Jensen, and Naser Khan. 
To

My Mother

Mrs. Abida Sultana 


\section{TABLE OF CONTENTS}

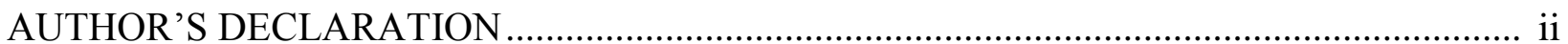

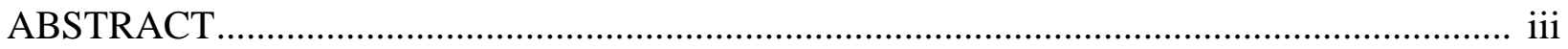

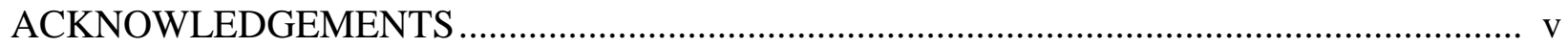

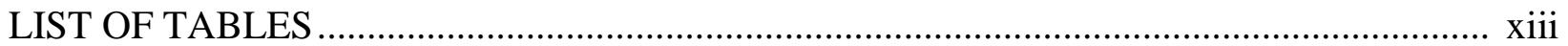

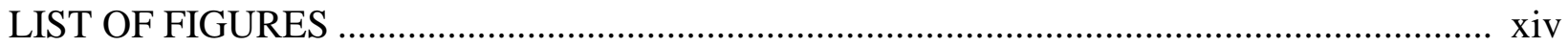

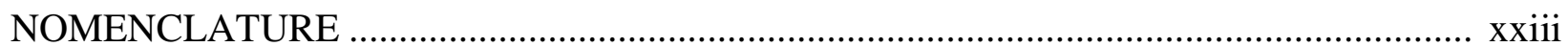

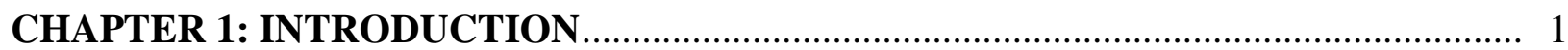

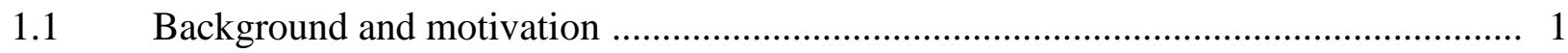

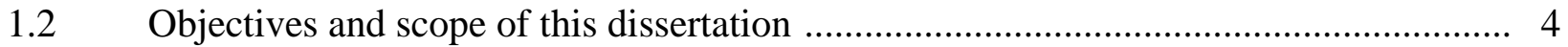

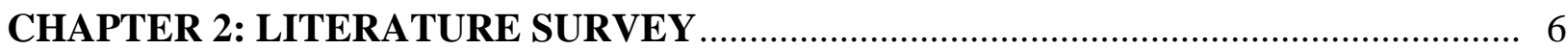

2.1 Current status of $\mathrm{Mg}$ alloy research and development ....................................... 6

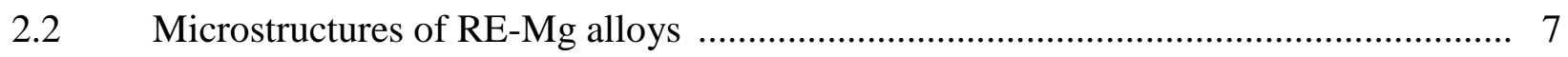

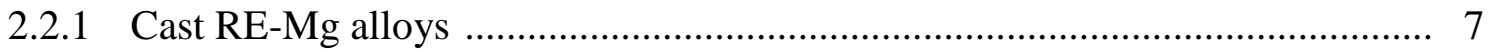

2.2.2 Extruded RE-Mg alloys ............................................................... 9

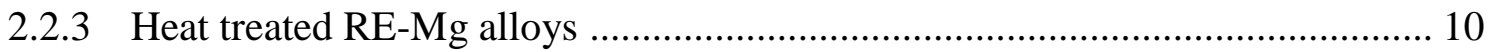

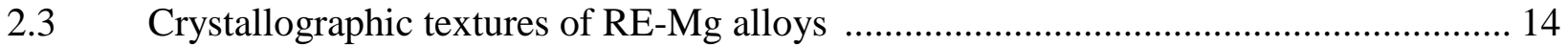

2.4 Tensile and compressive properties of RE-Mg alloys ........................................ 17

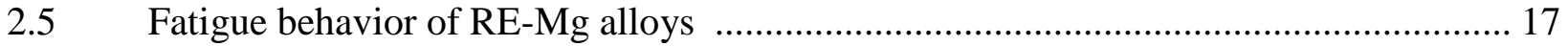

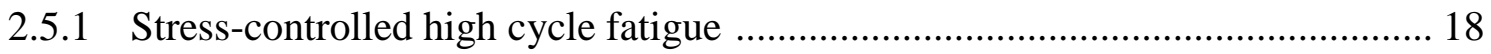

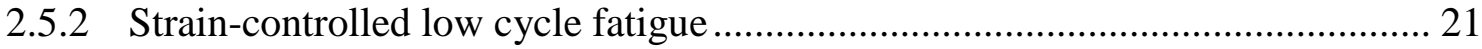

2.5.2.1 Stress-strain hysteresis loops _.................................................... 22 
2.5.2.2 Cyclic deformation response …………………………………….... 23

2.5.2.3 Fatigue life and parameters ……………………………………...... 24

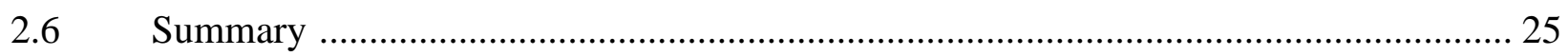

CHAPTER 3: EXPERIMENTAL PROCEDURE ............................................................ 27

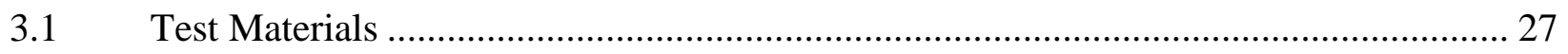

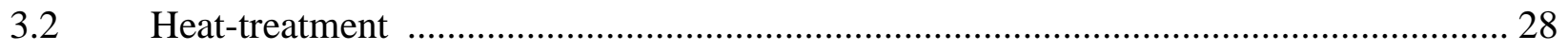

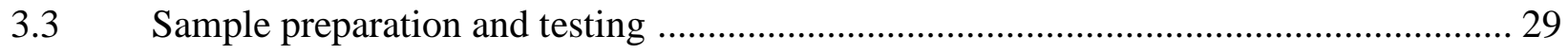

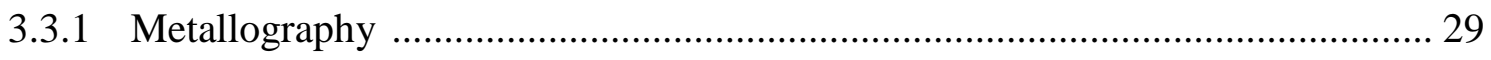

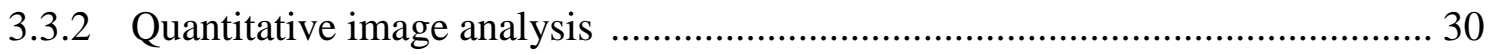

3.3.3 Phase identification by X-ray diffraction …………............................................ 30

3.3.4 Texture measurements by X-ray diffraction ……............................................. 31

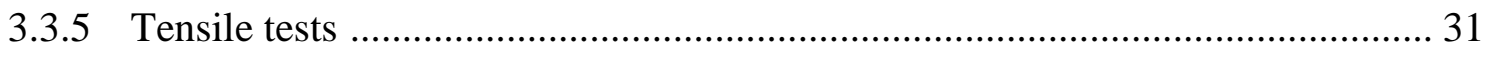

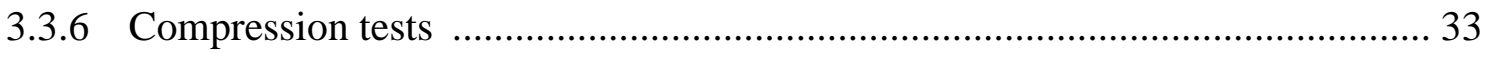

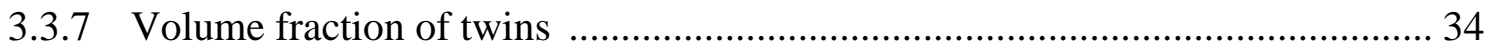

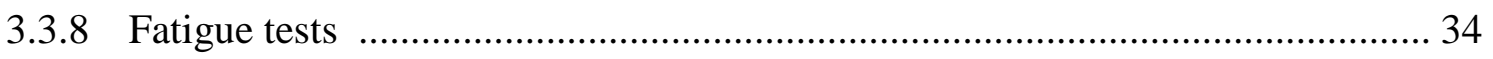

CHAPTER 4: DEFORMATION BEHAVIOR OF AN EXTRUDED GW103K ALLOY

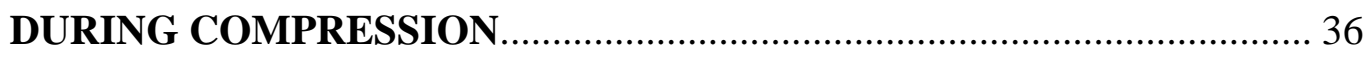

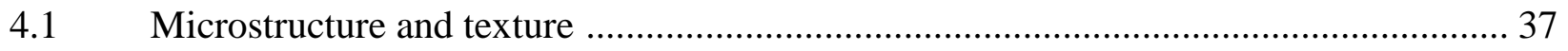

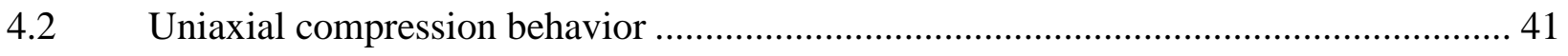

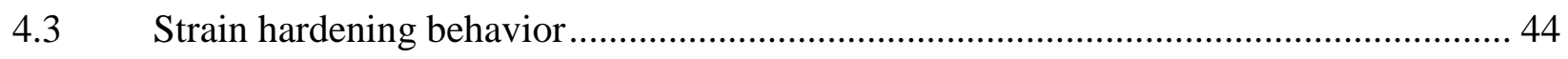

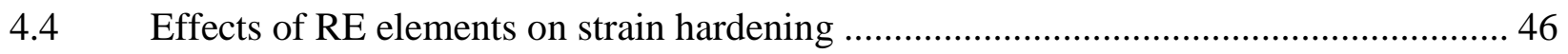

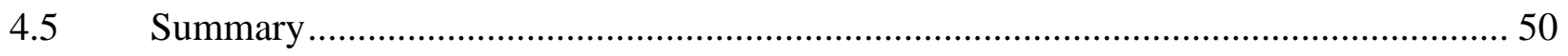


CHAPTER 5: A MODIFIED JOHNSON-COOK CONSTITUTIVE RELATIONSHIP FOR A RARE-EARTH CONTAINING MAGNESIUM ALLOY .............. 52

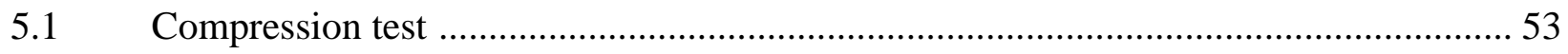

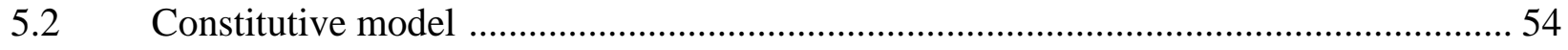

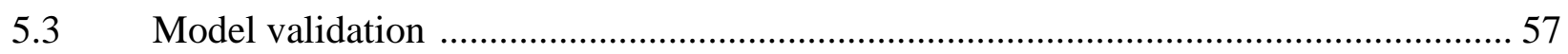

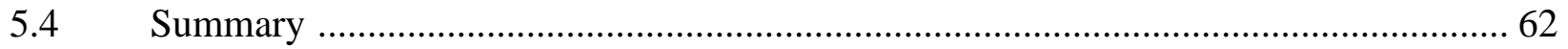

\section{CHAPTER 6: CYCLE DEFORMATION BEHAVIOR OF EXTRUDED GW103K}

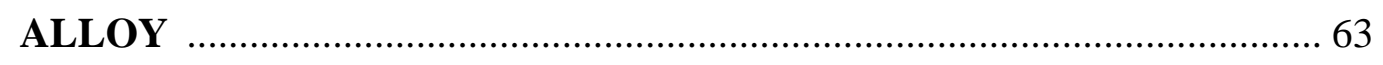

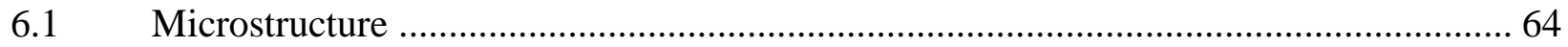

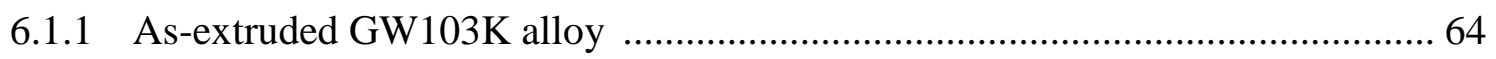

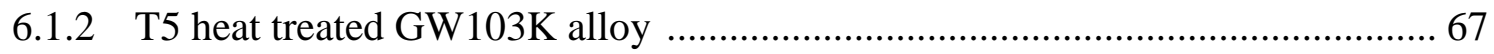

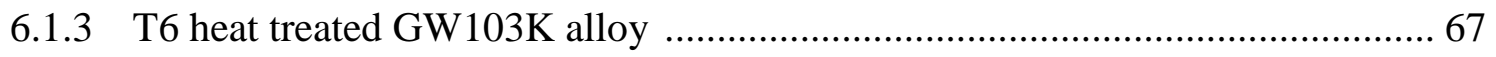

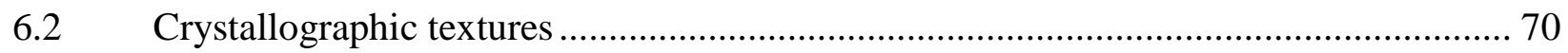

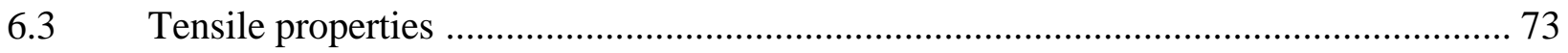

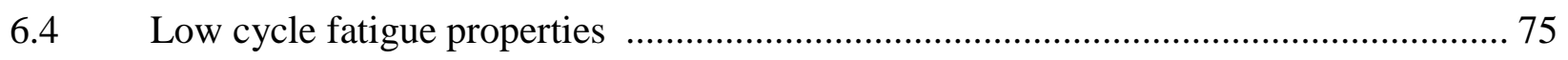

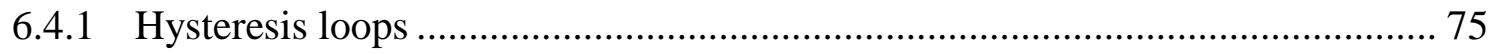

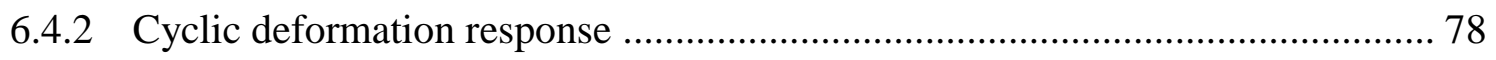

6.4.3 Fatigue life and fatigue parameters............................................................ 82

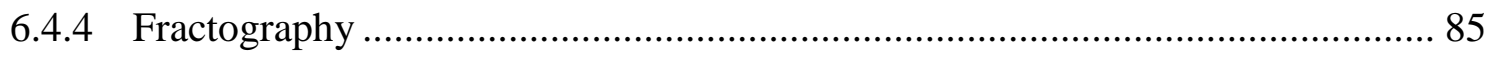

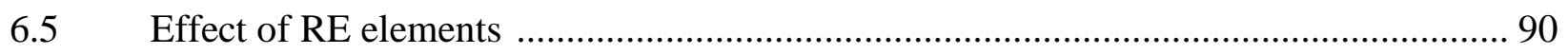

6.5.1 Effect on tensile properties ………………........................................... 90

6.5.2 Effect on cyclic deformation characteristics ................................................. 92

6.5.3 Interaction between twins and precipitates ..................................................... 96 
6.6 Summary 101

CHAPTER 7: EFFECT OF STRAIN RATIO ON CYCLIC DEFORMATION BEHAVIOR OF EXTRUDED GW103K ALLOY 103

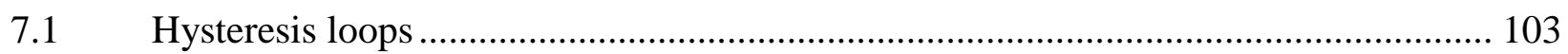

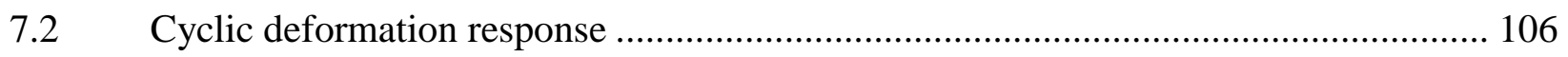

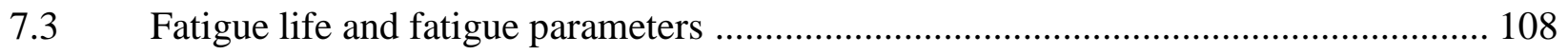

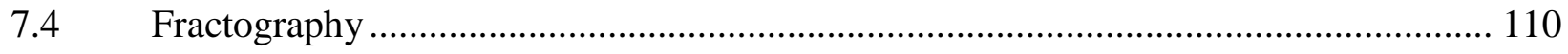

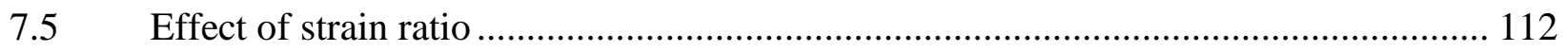

7.6 Comparison between GW103K alloy and RE-free extruded AM30 alloy ................... 113

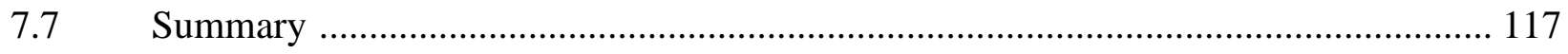

CHAPTER 8: CYCLIC DEFORMATION BEHAVIOR OF AN EXTRUDED NZ3OK

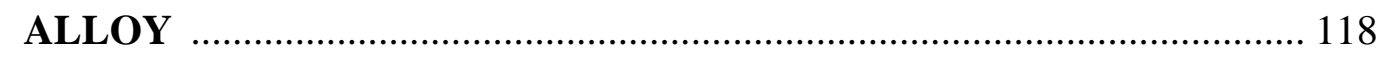

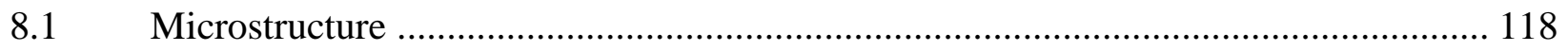

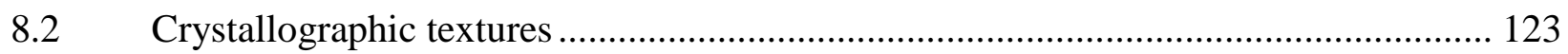

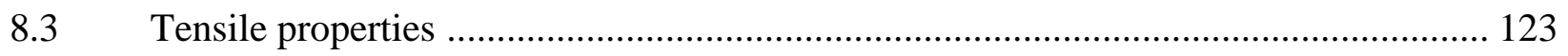

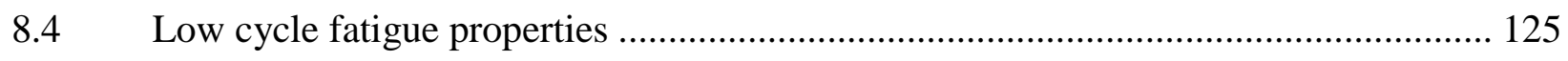

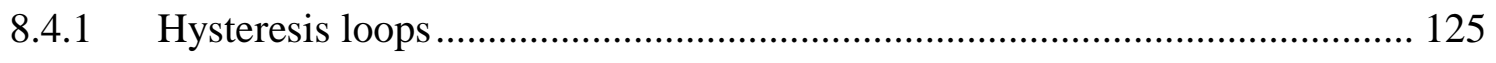

8.4.2 Cyclic deformation response ……………........................................... 126

8.4.3 Fatigue life and fatigue parameters ………................................................. 129

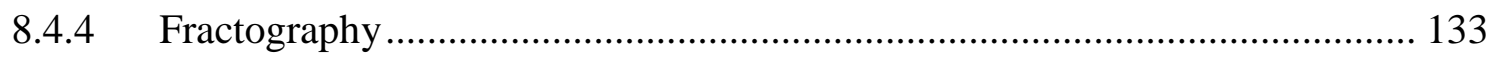

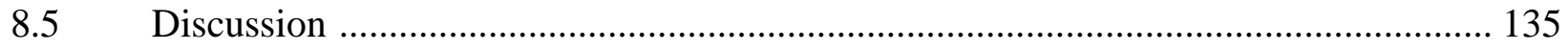

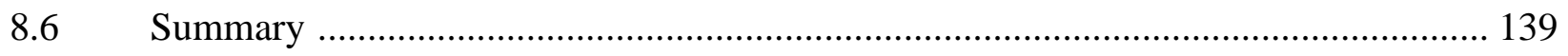

CHAPTER 9: SUMMARY, CONCLUSIONS AND FUTURE WORK …………............ 141 


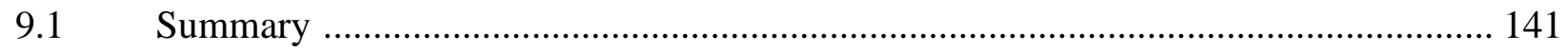

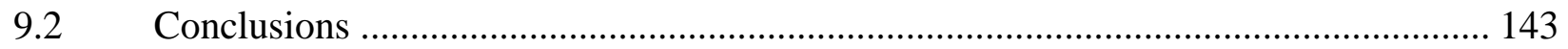

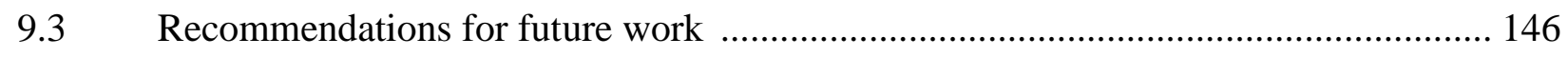

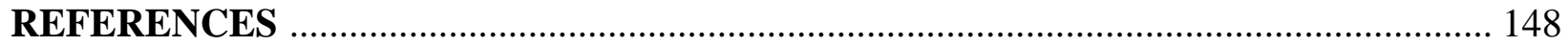




\section{LIST OF TABLES}

Table 3.1: Chemical composition (in wt.\%) of GW103K and NZ30K alloys.

Table 3.2: $\quad$ Test parameters under different strain ratios at a strain amplitude of $0.8 \%$ and a strain rate of $1 \times 10^{-2} \mathrm{~s}^{-1}$.

Table 4.1: Critical resolved shear stresses (CRSS) reported for $\mathrm{Mg}$ and its alloys. ......

Table 5.1: The material constants in the equations of Hollomon [210], Ludwik [211], and Afrin et al. [99] for the flow stress curves at different strain rates.

Table 5.2: A comparison between the predicted $\left(\sigma_{p}\right)$ and measured $\left(\sigma_{m}\right)$ flow stress values.

Table 6.1: Tensile properties of GW103K alloy in different states obtained at a strain rate of $1 \times 10^{-4} \mathrm{~s}^{-1}$.

Table 6.2: Low cycle fatigue parameters obtained for the GW103K alloy in the as-extruded, T5, and T6 conditions.

Table 8.1: Tensile properties of as-extruded NZ3OK and GW103K alloys obtained at a strain rate of $1 \times 10^{-4} \mathrm{~s}^{-1}$.

Table 8.2: Low cycle fatigue parameters obtained for the as-extruded NZ3OK alloy. .. 


\section{LIST OF FIGURES}

Figure 2.1: (a) Typical SEM micrographs of as-cast Mg-15Gd-5Y-0.5Zr alloy [77] and (b) as-cast Mg-8Gd-0.6Zr-xNd-yY alloys, $(x+y=3$, mass $\%)$ [70]. .....

Figure 2.2: SEM image of as-cast sample and the corresponding EDS spectra of the points indicated in the image [57].

Figure 2.3: Optical images of the as-extruded specimens: (a) and (b) Mg-8Gd-0.4Zr alloy, (c) and (d) Mg-8Gd-1Zn-0.4Zr alloy, and (e) and (f) Mg-8Gd-3 Zn-0.4Zr alloy [78].

Figure 2.4: Effects of solution treatment temperature on the microstructure of $M g-10$ Gd-2Y-0.5Zr alloy, whose specimens were solution-treated at the temperature indicated in photographs for $8 \mathrm{~h}$ [57].

Figure 2.5: XRD analysis of GW103 alloy under different conditions: (a) extruded $-F,(b)$ extruded-UA, (c) extruded-PA, (d) extruded-OA, (e) extruded-T4, and $(f)$ extruded-T6 [102].

Figure 2.6: Bright field images taken along $(a)[0001]_{\alpha}$ and $(b)[10 \overline{1} 0]_{\alpha}$ zone axis and corresponding SAED patterns shown in (c) B//[0001 $]_{\alpha}$ and $(d) B / /$ $[10 \overline{1} 0]_{\alpha}$ recorded from a $\mathrm{Mg}-10 \mathrm{Gd}-2 \mathrm{Y}-0.5 \mathrm{Zr}$ alloy aged at $225^{\circ} \mathrm{C}$ for $16 \mathrm{~h}[57]$.

Figure 2.7: Effect of $G d$ content on the recrystallization texture intensity in a Mg-Gd binary alloy [115].

Figure 2.8: (0001) and $\{10 \overline{1} 0\}$ pole figures of the as-received (a) AZ31 and (b) ZE1O sheets, where density profiles of the basal pole along different sheet 
directions are shown for (c) AZ31 and (d) ZE10 sheets (RD: rolling direction, TD: transverse direction, $45^{\circ}: 45^{\circ}$ from the RD) [123]........... 16

Figure 2.9: Uniaxial tensile and compressive stress-strain curves for (a) GW123k alloy and (b) AZ31 alloy [37]

Figure 2.10: S-N curves for an AZ91D alloy with different amounts of Ce addition, (a) $0 \% \mathrm{Ce},(b) 1 \% \mathrm{Ce}$, and (c) $2 \% \mathrm{Ce}[86,142]$.

Figure 2.11: S-N curve of an extruded Mg-12Gd-3Y-0.5Zr (GW123K) alloy [37].........

Figure 2.12: Stress-strain hysteresis loops of an extruded GW83 alloy fatigued at (a) $\Delta \varepsilon / 2=4.0 \%$, (b) $\Delta \varepsilon / 2=0.77 \%$, and (c) $\Delta \varepsilon / 2=0.30 \%[58]$.

Figure 2.13: Hysteresis loops of a GW123K alloy fatigued at varying total strain amplitudes [148]

Figure 2.14: Variation of stress amplitude with increasing number of cycles at varying strain amplitudes from $0.275 \%$ to $5.0 \%$ for an extruded GW83 alloys [58].

Figure 2.15: Total strain amplitude-fatigue life curves for GW102K and AZ31 alloys [149].

Figure 3.1: T6 heat treatment procedure for the RE-Mg alloys.

Figure 3.2: Geometry and dimensions of the sub-sized tensile/fatigue test specimens according to ASTM E8 [164].

Figure 3.3: Specimen dimension for the compression tests, prepared according to ASTM E 9M standards [165].

Figure 4.1: Microstructures of extruded (a) GW103K and (b) AM30 alloy.

Figure 4.2: (a) SEM backscattered electron image indicating EDS point analysis 
locations (A, B, and $C)$, (b) SEM backscattered electron image indicating EDS line scan position, and (c) the corresponding EDS line scan results in the GW103K alloy.

Figure 4.3: Transmission electron micrographs of the as-extruded GW103K alloy: (a) bright-field image taken along [001] zone axis of particle A, and (b) [001] zone axis microdiffraction pattern recorded from particle A

Figure 4.4: (0002) pole figure of as-extruded GW103K alloy, where ED stands for the extrusion direction and $R D$ indicates the radial direction.

Figure 4.5: (a) Compressive stress-strain curves and (b) strain hardening rate as a function of true strain of as-extruded $G W 103 \mathrm{~K}$ alloy in the extrusion direction tested at a strain rate of $10^{-3} \mathrm{~s}^{-1}$.

Figure 4.6: Variation of the volume fraction of twins with the applied strain during compression in the as-extruded GW103K alloy, where some typical micrographs at different strain levels are inserted to show the evolution of deformation twins

Figure 4.7: Typical SEM micrographs of as-extruded GW103K alloy deformed in compression at a strain level of (a) $3.2 \%$, and (b) $12.3 \%$.

Figure 5.1: Compressive stress-strain curves of the extruded GW103K alloy and REfree AM30 alloy [108].

Figure 5.2: Comparison of the calculated true stress-strain curves of the extruded GW103K alloy with those experimentally determined at different strain rates of (a) $0.1 \mathrm{~s}^{-1}$, (b) $0.01 \mathrm{~s}^{-1}$, (c) $0.001 \mathrm{~s}^{-1}$, and (d) $0.0001 \mathrm{~s}^{-1}$.

Figure 6.1: Microstructures (left optical micrographs and right SEM back-scattered 
electron images) of the GW103K alloy in the (a) and (b) as-extruded, (c)

and $(d)$ T5, and (e) and (f) T6 states.

Figure 6.2: SEM back-scattered electron images indicating EDS line scan positions and the corresponding EDS line scan results of $G W 103 \mathrm{~K}$ alloy the (a) and (b) as-extruded, (c) and (d) T5, and (e) and (f) T6 conditions.

Figure 6.3: Transmission electron micrographs recorded from a sample of T6 alloy:

(a) bright-field image taken along [001] zone axis of particle A, (b) [001] zone axis microdiffraction pattern recorded from particle A, (c) brightfield images taken along [0001] $]_{\alpha}$ zone axis, and (d) the corresponding SAED patterns shown in B//[0001]

Figure 6.4: Pole figures of basal (0001) plane, prismatic (10 $\overline{1} 0)$ plane, and pyramidal (101 1) plane of GW103K alloy in the (a) as-extruded, (b) T5, and (c)T6 states, where ED indicates the extrusion direction and RD denotes the radial direction.

Figure 6.5: Typical tensile stress-strain curves of the GW103K alloy in different conditions tested at a strain rate of $1 \times 10^{-4} \mathrm{~s}^{-1}$

Figure 6.6: Typical stress-strain hysteresis loops of GW103K alloy in different states tested at a given total strain amplitude of $1.2 \%$ and strain ratio of $R_{\varepsilon}=-1$ of (a) first cycle, (b) second cycle, and (c) mid-life cycle.

Figure 6.7: Stress amplitude vs. the number of cycles at different total strain amplitudes applied for the GW103K alloy in the (a) as-extruded, (b) T5, and (c) T6 states.

Figure 6.8: Plastic strain amplitude vs. the number of cycles at different total strain 
amplitudes applied for the GW103K alloy in the (a) as-extruded, (b) T5, and (c) T6 states.

Figure 6.9: Total strain amplitude versus the number of cycles to failure for the GW103K alloy in the as-extruded, T5, and T6 states, in comparison with the data reported in the literature for various extruded Mg alloys [26,28, 156-159].

Figure 6.10: Cyclic stress-strain curves for the GW103K alloy in the (a) as-extruded, (b) T5, and (c) T6 states, where the corresponding monotonic stress-strain curves are also plotted for comparison.

Figure 6.11: An overall view of fracture surfaces fatigued at a strain amplitude of $0.4 \%$ of the GW103K alloy in the (a) as-extruded, (b) T5, and (c) T6 states.....

Figure 6.12: SEM micrographs of fracture surfaces near crack initiation area (left) and in the propagation zone (right) of the GW103K alloy in the (a) and (b) asextruded, $(c)$ and $(d) T 5$, and $(e)$ and $(f)$ T6 states fatigued at a strain amplitude of $0.4 \%$.

Figure 6.13: SEM micrographs of the fracture surface of the T6 sample fatigued at a strain amplitude of $0.4 \%$ in the region near the upper left corner $((a)$ and (b)), and region near the lower right corner $((c)$ and $(d))$ as indicated by the yellow dashed boxes in Fig.11(c).

Figure 6.14: Typical stress-strain hysteresis loops of the mid-life cycle at a given total strain amplitude of $1.2 \%$ and strain ratio of $R_{\varepsilon}=-1$ for the extruded AM30 and $G W 103 K$ alloy in different states, respectively.

Figure 6.15: Optical micrographs in the area near the fracture surface at a strain 
amplitude of $1.2 \%$, showing the distribution of deformation features in the fatigued samples of (a) AM30 alloy, (b) as-extruded, (c) T5, and

(d) T6 states of the $G W 103 \mathrm{~K}$ alloy, respectively.

Figure 6.16: A schematic illustration of the interaction between a plate-shaped precipitate and $\{10 \overline{1} 2\}$ twin in the GW103K alloy.

Figure 6.17: A schematic illustration of the interaction between (1 $\overline{1} 02)$ twin and $\beta^{\prime}$ precipitate with an orientation relationship of $[100]_{\beta^{\prime}} / /[2 \overline{1} \overline{1} 0]_{\alpha}$ and $[001]_{\beta^{\prime}} / /[0001]_{\alpha}$ in the GW103K alloy. 99

Figure 7.1: Typical stress-strain hysteresis loops of different cycles at different strain ratios at a given total strain amplitude of $0.8 \%$ of the extruded $G W 103 \mathrm{~K}$ alloy, (a) First cycle, (b) Second cycle, and (c) Mid-life cycle.

Figure 7.2: Stress amplitude vs. the number of cycles at different strain ratios at a given total strain amplitudes of $0.8 \%$ of the extruded $G W 103 \mathrm{~K}$ alloy........

Figure 7.3: Plastic strain amplitude vs. the number of cycles at different strain ratios at a given total strain amplitudes of $0.8 \%$ of the extruded GW103K alloy.

Figure 7.4: Number of cycles to failure vs. strain ratio for the extruded GW103K alloy tested at a given total strain amplitude of $0.8 \%$ and strain rate of $1 \times 10^{-2} s^{-1}$

Figure 7.5: Mean stress vs. a normalized cycle ratio $\left(N / N_{f}\right)$ at different strain ratios in the $G W 103 K$ alloy tested at a total strain amplitudes of $0.8 \%$.

Figure 7.6: Stress ratio of the first cycle and the mid-life cycle as a function of the applied strain ratio.

Figure 7.7: Typical SEM images of overall fracture surfaces of the extruded GW103K 
specimens fatigued at a total strain amplitude of $0.8 \%$ and at a strain ratio of (a) 0.5 and (b) $-\infty$

Figure 7.8: SEM micrographs of the fracture surface near crack initiation of the extruded GW103K specimens fatigued at a total strain amplitude of $0.8 \%$ and at a strain ratio of (a) 0.5 and $(b)-\infty$.

Figure 7.9: SEM micrographs of fatigue crack propagation region of the extruded GW103K specimens fatigued at a total strain amplitude of $0.8 \%$ and at a strain ratio of (a) 0.5 and $(b)-\infty$

Figure 7.10: Typical stress-strain hysteresis loops of (a) first and (b) mid-life cycles at different strain ratios at a total strain amplitude of $0.8 \%$ in the $G W 103 K$ alloy (solid lines) and AM30 alloy (dashed lines).

Figure 7.11: Optical micrographs in the areas near the fracture surface at a strain amplitude of $0.8 \%$, showing the distribution of residual twins in the fatigued samples of $G W 103 \mathrm{~K}$ alloy $\left((a) R_{\varepsilon}=0.5\right.$, (c) $R_{\varepsilon}=-1$, and $\left.(e) R_{\varepsilon}=-\infty\right)$ and AM30 alloy $\left((b) R_{\varepsilon}=0.5,(d) R_{\varepsilon}=-1\right.$, and $\left.(f) R_{\varepsilon}=-\infty\right)$, respectively. .......

Figure 8.1: Microstructures of extruded NZ3OK alloy, (a) and (c) OM images, and (b) and (d) SEM back-scattered electron images.

Figure 8.2: SEM back-scattered electron images indicating (a) EDS line scan positions and (b) the corresponding EDS line scan results of NZ3OK alloy.

Figure 8.3: $\quad$ X-ray diffraction pattern of extruded NZ30K alloy.

Figure 8.4: Transmission electron micrographs recorded from a sample of as-extruded NZ30K alloy: (a) bright-field image, and (b) corresponding SAED patterns, where beam line was parallel to $\langle 11-20\rangle_{M g}$. 
Figure 8.5: Pole figures of basal (0001) plane, prismatic (10 $\overline{1} 0)$ plane, and pyramidal (10 $\overline{1} 1)$ plane of extruded NZ3OK alloy, where ED stands for the extrusion direction and $R D$ indicates the radial direction.

Figure 8.6: Typical tensile stress-strain curves of extruded NZ3OK and GW103K alloys tested at strain rate of $1 \times 10^{-4} \mathrm{~s}^{-1}$

Figure 8.7: Typical stress-strain hysteresis loops of different cycles at a total strain amplitude of $1.2 \%$ and strain ratio of $R_{\varepsilon}=-1$ for the extruded $A M 30$ and NZ30K alloys, respectively.

Figure 8.8: Stress amplitude vs. the number of cycles for different total strain amplitudes tested at a strain ratio of $R_{\varepsilon}=-1$ of extruded $N Z 30 K$ alloy. ......

Figure 8.9: Plastic strain amplitude vs. the number of cycles for different total strain amplitudes tested at a strain ratio of $R_{\varepsilon}=-1$ of extruded NZ3OK alloy. ......

Figure 8.10: Total strain amplitude as a function of the number of cycles to failure for the extruded NZ3OK alloy, in comparison with the data reported in the literature for various extruded Mg alloys [26,28,156-159].

Figure 8.11: Cyclic total, elastic, and plastic strain amplitudes vs. number of reversals to failure of the extruded NZ3OK alloy.

Figure 8.12: Cyclic stress-strain curve for extruded NZ3OK alloy, where the corresp-onding monotonic stress-strain curve is plotted for comparison.

Figure 8.13: SEM images of overall fracture surfaces of the extruded NZ3OK specimens fatigued at a total strain amplitude of (a) $0.4 \%$ and (b) $1.0 \%$.

Figure 8.14: SEM micrographs of the fracture surface near crack initiation of the extruded NZ30K specimens fatigued at a total strain amplitude of (a) 0.4\%, 
and (b) $1.0 \%$.

Figure 8.15: SEM micrographs (left: SEM images and right: SEM back-scattered electron images) of the fatigue crack propagation region of the extruded NZ30K specimens fatigued at a total strain amplitude of (a) and (b) $0.4 \%$ and (c) and (d) $1.0 \%$.

Figure 8.16: Typical stress-strain hysteresis loops of the mid-life cycle at a given total strain amplitude of $1.2 \%$ and strain ratio of $R_{\varepsilon}=-1$ for the extruded NZ3OK, GW103K, and AM30 alloys, respectively.

Figure 8.17: Optical micrographs in the area near the fracture surface at a strain amplitude of $1.2 \%$, showing the distribution of deformation twins in the fatigued samples of (a) AM30, (b) as-extruded GW103K, and (c) asextruded NZ3OK alloys, respectively. 


\section{NOMENCLATURE}

\begin{tabular}{|c|c|}
\hline Acronym & Definition \\
\hline ASTM & American Society for Testing of Materials \\
\hline USAMP & United States Automotive Materials Partnership \\
\hline bcc & Body centered cubic \\
\hline fcc & Face centered cubic \\
\hline hep & Hexagonal closed packed \\
\hline $\mathrm{HCF}$ & High cycle fatigue \\
\hline LCF & Low cycle fatigue \\
\hline $\mathrm{Mg}$ & Magnesium \\
\hline $\mathrm{Al}$ & Aluminum \\
\hline $\mathrm{Mn}$ & Manganese \\
\hline $\mathrm{Cu}$ & Copper \\
\hline $\mathrm{Zn}$ & Zinc \\
\hline $\mathrm{Fe}$ & Iron \\
\hline $\mathrm{Ni}$ & Nickel \\
\hline RT & Room temperature \\
\hline SEM & Scanning electron microscope \\
\hline EDS & Energy dispersive X-ray spectroscopy \\
\hline TEM & Transmission electron microscope \\
\hline T5 & Artificially aged \\
\hline T6 & Solution heat treated and artificially aged \\
\hline$\% \mathrm{El}$ & $\%$ Elongation \\
\hline
\end{tabular}


UTS

YS

RE

\section{Symbol}

$\alpha, \beta$

$\sigma$

$\varepsilon$

$\dot{\varepsilon}$

$n$

K

E

$\theta$

$\left(\frac{\Delta \varepsilon_{t}}{2}\right)$

$\left(\frac{\Delta \varepsilon_{e}}{2}\right)$

$\left(\frac{\Delta \varepsilon_{\mathrm{p}}}{2}\right)$

$N$

$N_{f}$

$n^{\prime}$

$K^{\prime}$
Ultimate tensile strength

Yield strength

Rare-earth

\section{Definition}

Phase designations

Stress, MPa

Strain

Strain rate, $\mathrm{s}^{-1}$

Strain hardening exponent

Strength coefficient, MPa

Young's modulus or modulus of elasticity, MPa

Strain or work hardening rate, $\mathrm{MPa}$

Total strain amplitude

Elastic strain amplitude

Plastic strain amplitude

Number of cycles

Number of cycles to failure

Cyclic strain hardening exponent

Cyclic strength coefficient, MPa 
$\sigma_{f}^{\prime}$

$b$

$\varepsilon_{f}^{\prime}$

$c$

$R$

$R_{\varepsilon}$
Fatigue strength coefficient, $\mathrm{MPa}$

Fatigue strength exponent

Fatigue ductility coefficient, $\%$

Fatigue ductility exponent

Stress ratio

Strain ratio 


\section{CHAPTER 1}

\section{INTRODUCTION}

\subsection{Background and motivation}

The increasing climate extremes such as severe droughts, worrisome water risks, superstorms and destructive floods under global warming, which are known to be largely irreversible on timescales of many centuries [1], have today been recognized to be a consequence of the anthropogenic greenhouse gas emissions [2-10]. Lightweighting of ground vehicles at present is regarded as one of the most effective strategies to improve fuel economy and reduce environment-damaging, climate-changing, human death-causing ${ }^{1}$ and costly $^{2}$ emissions [11-14]. It has been reported that the fuel efficiency of passenger vehicles can be enhanced by $6-8 \%$ for each $10 \%$ reduction in weight [15]. Advanced high-strength steels, aluminum alloys, magnesium $(\mathrm{Mg})$ alloys, and polymers are being used to reduce vehicle weight and the subsequent emissions [16,17], but substantial reductions could be achieved further by more applications of Mg alloys which have been considered as a strategic ultra-lightweight material in the automotive and aerospace sectors [11,12,18-23].

In the structural applications of $\mathrm{Mg}$ alloys in the transportation industry, including camshaft covers, clutch and transmission housings, intake manifolds and automobile wheels [24], the

\footnotetext{
${ }^{1}$ According to Science News entitled "Air pollution kills 7 million people a year" on March 25, 2014 at http://news.sciencemag.org/signal-noise/2014/03/air-pollution-kills-7-million-people-year: "Air pollution isn't just harming Earth; it's hurting us, too. Startling new numbers released by the World Health Organization today reveal that one in eight deaths are a result of exposure to air pollution. The data reveal a strong link between the tiny particles that we breathe into our lungs and the illnesses they can lead to, including stroke, heart attack, lung cancer, and chronic obstructive pulmonary disease."

${ }^{2}$ Revesz RL, Howard PH, Arrow K, Goulder LH, Kopp RE, Livermore MA, Oppenheimer M, Sterner T, Global warming: Improve economic models of climate change, Nature, April 10, 2014, vol.508, pp.173-175.
} 
components are inevitably subjected to cyclic stresses and strains for millions of cycles in service [16,25-32]. It is well known that a metal subjected to repeated or fluctuating stresses will fail at a stress level much lower than that required to cause fracture on a single application of load [33]. Such a failure occurring under conditions of dynamic loading is called fatigue failure. It accounts for about $80 \sim 90 \%$ of all service failures due to mechanical stresses [34]. Fatigue failure is particularly harmful because it occurs without any obvious warning. Hence, knowledge on the cyclic deformation and fatigue behavior of $\mathrm{Mg}$ alloys is of vital importance for the design and durability evaluation of structural engineering components.

Despite the potential of substantial reductions in weight, most wrought $\mathrm{Mg}$ alloys exhibited a high degree of anisotropy and tension-compression yield asymmetry due to the presence of strong crystallographic texture owing to their hexagonal close-packed (HCP) crystal structure with limited slip systems during extrusion or rolling processes [25-28,32,35-41]. Indeed, for the vehicle components subjected to dynamic cyclic loading, such mechanical anisotropy and tension-compression yield asymmetry could lead to irreversibility of cyclic deformation which may have an unfavorable influence on the performance and durability of structural components. Since there is a strong correlation between the crystallographic texture evolution during extrusion or rolling processes, the operating slip and twinning, and the resulting mechanical behavior, it is expected that controlling the microstructure, i.e., texture and grain size, has the potential to conquer the problems with the mechanical anisotropy and tension-compression yield asymmetry [42]. One appealing approach of achieving this goal is via alloy composition adjustments, especially the addition of rare-earth (RE) elements into 
$\mathrm{Mg}$ alloys [37,42-56]. The addition of RE elements in Mg alloys can lead to fairly random initial crystallographic texture compared with the RE-free wrought $\mathrm{Mg}$ alloys, which leads to improved ductility and strength at both room and elevated temperatures via solid solution strengthening and precipitation strengthening [57-61]. It was also reported that a RE element alternates the bonding energy between $\mathrm{Mg}$ atoms and $\mathrm{RE}$ atoms, thus increasing the possibility of non-basal slip and inhibiting the basal slip and twinning [58]. For example, Stanford and Barnett [60] reported that the anisotropy of yield strength of a rolled Mg-Zn-RE alloy was reversed as compared with the traditional $\mathrm{Mg}-\mathrm{Zn}$ alloy. Although these alterations in the tension-compression yield asymmetry due to the RE elements additions are being gradually enlightened, the detailed information of such RE-Mg alloys as structural components under dynamic cyclic loading condition is still lacking. It is unclear what are the effects of RE-elements on the tensile-compressive yield asymmetry which generally occurs in the RE-free extruded Mg alloys, whether RE-Mg alloys exhibit cyclic hardening or softening, to what extent the RE-elements affect the cyclic deformation behavior and fatigue life, and what would be the effects of the heat treatment on overall deformation behavior. Thus, proper understanding about the cyclic deformation characteristics of RE-containing $\mathrm{Mg}$ alloys is critical to harness the full potential and benefits of these alloys $[16,62]$. This dissertation represents a contribution to the $\mathrm{Mg}$ alloy development effort in the way of further enhance the understanding of the cyclic deformation behavior by examining the influence of grain size, texture, and twin formation in RE-Mg alloys. 


\subsection{Objectives and scope of this dissertation}

The general objective of this dissertation was to achieve a clear understanding of the deformation behavior of RE-Mg alloys. As mentioned earlier, studies on the alteration in the tension-compression yield asymmetry due to the addition of RE elements is being increasingly enlightened, clear understanding of the core mechanisms of deformation behavior of RE-Mg alloys is limited to the date. Therefore, as a first step, the compressive deformation behavior of such alloys was investigated followed by modeling of constitutive equation to predict the flow stress of RE-Mg alloys and lastly the cyclic deformation behavior was studied. The specific objectives of this present dissertation include the following:

- To identify the effect of RE elements on deformation behavior of an extruded Mg10Gd-3Y-0.5Zr (GW103K) alloy during compression at different strain levels and explores a constitutive relation to quantify its deformation behavior.

- To evaluate the cyclic deformation behavior of extruded and heat treated GW103K alloys under varying strain amplitudes and varying strain ratios.

- To explore the cyclic deformation behavior of an extruded $\mathrm{Mg}-3 \mathrm{Nd}-0.2 \mathrm{Zn}-0.5 \mathrm{Zr}$ (NZ30K) alloy under varying strain amplitudes.

The approach taken for meeting these goals was mostly experimental. This dissertation has been structured as follows:

In the Chapter 2, a literature survey is presented on the recent trend of research and development on RE-Mg alloys, their properties; the microstructural characterization and evolution of mechanical properties of different RE-Mg alloys after monotonic and cyclic 
deformation tests are also included. Chapter $\mathbf{3}$ gives the experimental procedures involved in this dissertation. Chapter 4 presents the details of the effect of RE elements on the deformation behavior of extruded GW103K alloy during compression at different strain levels with particular attention on the twin formation. Chapter $\mathbf{5}$ describes a constitutive relationship related to the stress, strain, temperature and strain rate to quantify the deformation behavior of extruded GW103K alloy based on the Johnson-Cook equation and a recently adopted strain hardening method. Chapters 6-8 presents the experimental results along with the discussion obtained from the low cycle fatigue tests subjected to different strain amplitudes of extruded GW103K alloy in different heat treatment states, and low cycle fatigue tests subjected to different strain ratios of extruded GW103K alloy, and low cycle fatigue tests subjected to different strain amplitudes of extruded NZ30K alloy. The conclusions stemming from the dissertation and points to the potential future scope for continuing with this research are stated in Chapter 9. 


\section{CHAPTER 2}

\section{LITERATURE SURVEY}

\subsection{Current status of $\mathrm{Mg}$ alloy research and development}

$\mathrm{Mg}$, discovered in 1774 and named after the ancient city Magnesia, is found to be the 6th most abundant element, constituting $2 \%$ of the total mass of the Earth's crust [16]. It can be produced from seawater, brines and Mg-bearing minerals which give a virtually unlimited supply of ore reserves. For example, it was estimated that at current world use levels there would be enough $\mathrm{Mg}$ in the Dead Sea for at least 22,000 years [63]. Mg is the lightest (density of $1.74 \mathrm{~g} / \mathrm{cm} 3, \sim 36 \%$ less than aluminum, $\sim 4.5$ times less than steel, and nearly the same as many polymers) structural metallic materials available $[11,64]$. The melting point and specific heat of $\mathrm{Mg}$ are almost the same as those of aluminum, while the Young's modulus and shear modulus of $\mathrm{Mg}$ are about two-thirds of those of aluminum. Pure $\mathrm{Mg}$ shows even higher damping properties than cast iron, although these properties are highly dependent on the prior heat treatment. Other attractive properties of $\mathrm{Mg}$ alloys include electromagnetic shielding, dimensional stability, and good machinability and recyclability [65]. Since 1993 there has been renewed interest in using "the forgotten material" $\mathrm{Mg}$ and Mg based alloys. In particular, the past decade has seen a growing demand for $\mathrm{Mg}$ alloy development for structural and automotive applications and other household and sport applications due to their large potential for weight saving and, thereby, for improved fuel economy and decreased exhaust emissions [19,65,66]. The uses of $\mathrm{Mg}$ as a structural material were, however, very few. The bulk was used as an alloying element in different 
alloys. Alloying Mg with aluminum, manganese, rare-earth, thorium, zinc or zirconium increases the strength to weight ratio making them important materials for applications where weight reduction is important, and where it is imperative to reduce inertial forces. Because of this property, denser material, not only steels, cast iron and copper base alloys, but even aluminum alloys are being considered to be replaced by Mg-based alloys $[63,67]$.

Furthermore, a report released by USAMP (United States Automotive Materials Partnership) expressed the vision of increasing considerably the use of $\mathrm{Mg}$ alloys by $340 \mathrm{lbs}$ per car by the year of 2020, which will lead to a significant reduction of the vehicle weight (about $15 \%$ ) [68]. In a October 2013 press release at WWJ Auto Summit, it was reported that "Vehicle lightweighting is auto industry's best opportunity to achieve CAFE (Corporate Average Fuel Economy) standard" and it was also stated that by using the modern technologies with lightweight materials the mass of every vehicle can be reduce by more than 95 kilograms (approximately 209 pounds), which is essentially the environmental equivalent of planting about 19 trees. Therefore, the application of $\mathrm{Mg}$ alloys results in weight reduction, energysaving, and as well as essentially equivalent to environmental protection.

\subsection{Microstructures of RE-Mg alloys}

\subsubsection{Cast RE-Mg alloys}

A lot of investigations have recently been conducted on the cast $\mathrm{Mg}$ alloys with the addition of RE elements [43,57,69-92]. These studies have shown that RE elements can be used to 
adjust mechanical properties with a wide range of alloy composition and heat treatment due to their fairly large solubility at the eutectic temperature and the formation of precipitates like $\mathrm{Mg}_{5} \mathrm{RE}$ (Gd, Y). Fig. 2.1 shows a typical microstructure of as-cast Mg-15Gd-5Y-0.5Zr alloy [77] and as-cast Mg-8Gd-0.6Zr-xNd-yY alloys, (x+y=3, wt.\%) [70], respectively, where the as-cast alloy is mainly composed of continuous rosette-shaped equiaxed dendrites, and partially interdendritic eutectic co-exists in the alloy. There are also many dark petal-like Zrrich regions that are dispersed in the $\alpha-\mathrm{Mg}$. According to the previous literature $[80,82,83,93,94]$, the main intermetallic compounds were $\mathrm{Mg}_{5} \mathrm{RE}, \mathrm{Mg}_{41} \mathrm{RE}_{5}$, and $\mathrm{Mg}_{24} \mathrm{RE}_{5}$. The average equiaxed interdendritic arm spacing was about $30 \mu \mathrm{m}$ in the as-cast $\mathrm{Mg}$ - $15 \mathrm{Gd}-$ $5 \mathrm{Y}-0.5 \mathrm{Zr}$ alloy [70], which is fairly small in comparison with the common $\mathrm{Mg}$, such as AZ31 and AM30 [25-28,35]. This was due to the role of added RE elements and zirconium, where $\mathrm{Zr}$ mainly restricted the grain growth [95]. In addition, according to the study by He et al. [57], as-cast $\mathrm{Mg}-10 \mathrm{Gd}-2 \mathrm{Y}-0.5 \mathrm{Zr}$ alloy contains three different phases: $\alpha-\mathrm{Mg}$ solid solution matrix phase with supersaturated $\mathrm{Gd}+\mathrm{Y}$ elements, $(\mathrm{Gd}+\mathrm{Y})$-rich eutectic compound which had a higher Gd+Y content than the matrix, and intracrystalline Zr-rich cores (Fig. 2.2).
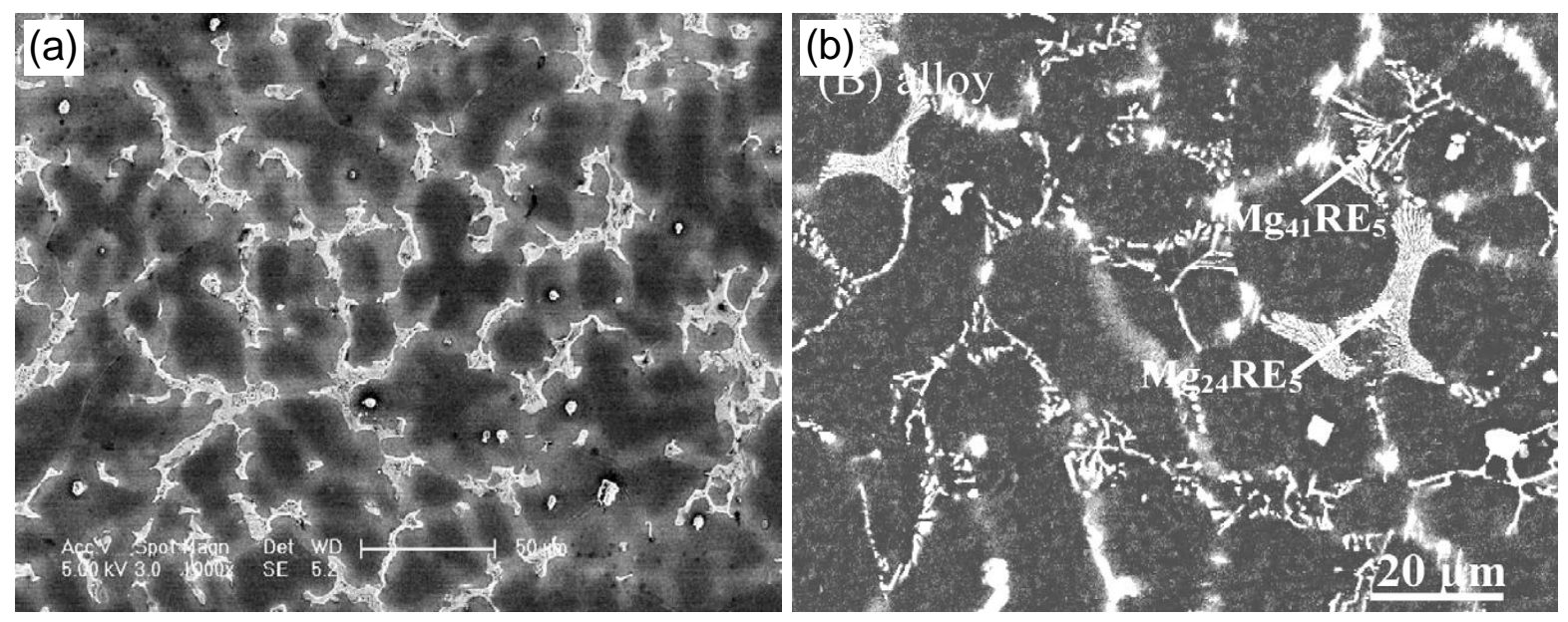

Figure 2.1: (a) Typical SEM micrographs of as-cast Mg-15Gd-5Y-0.5Zr alloy [77] and (b) as-cast $M g-8 G d-0.6 Z r-x N d-y Y$ alloys, $(x+y=3$, mass\%) [70]. 

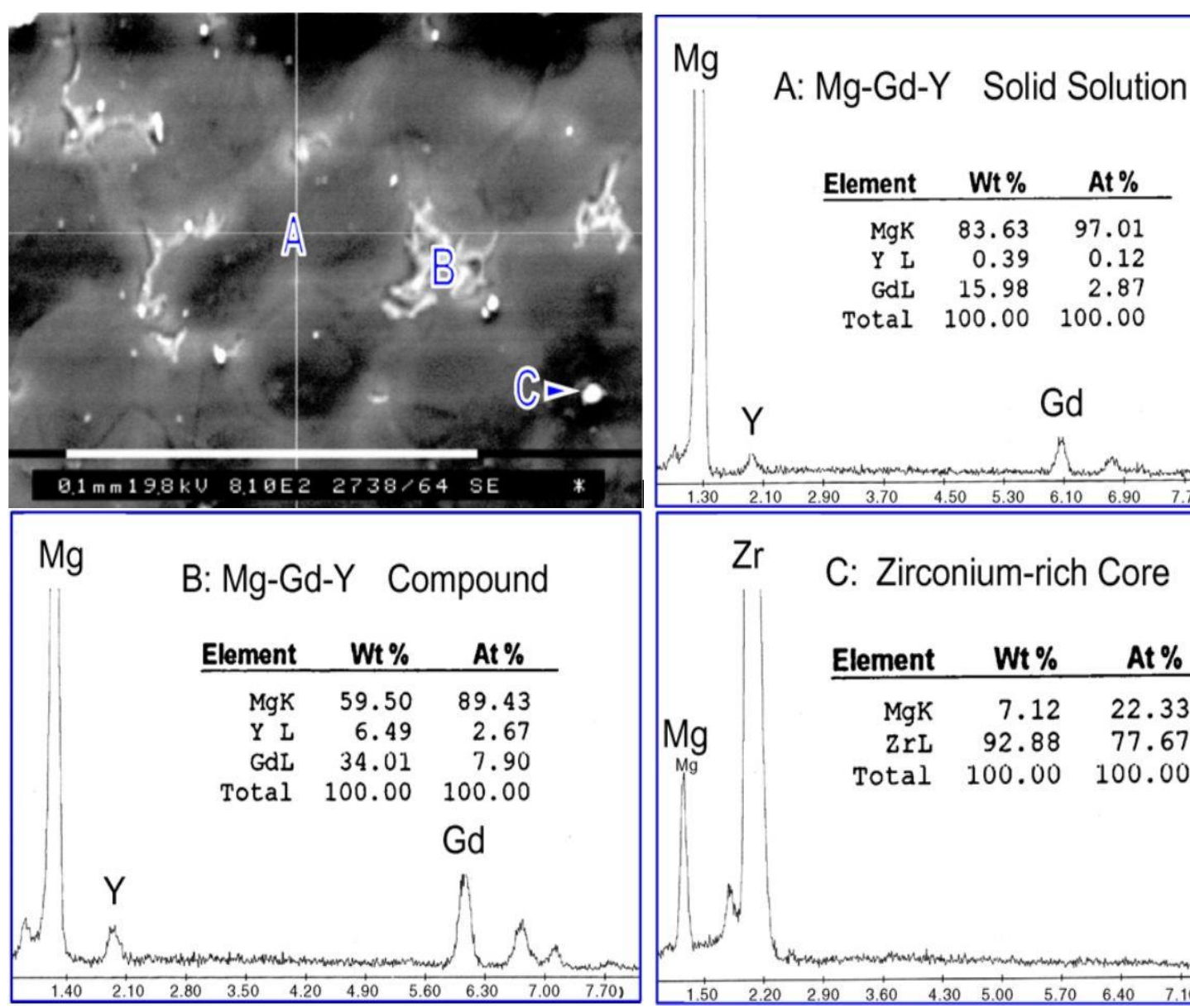

\begin{tabular}{|rrr} 
Element & Wt \% & At \% \\
\hline MgK & 83.63 & 97.01 \\
Y L & 0.39 & 0.12 \\
GdL & 15.98 & 2.87 \\
Total & 100.00 & 100.00
\end{tabular}
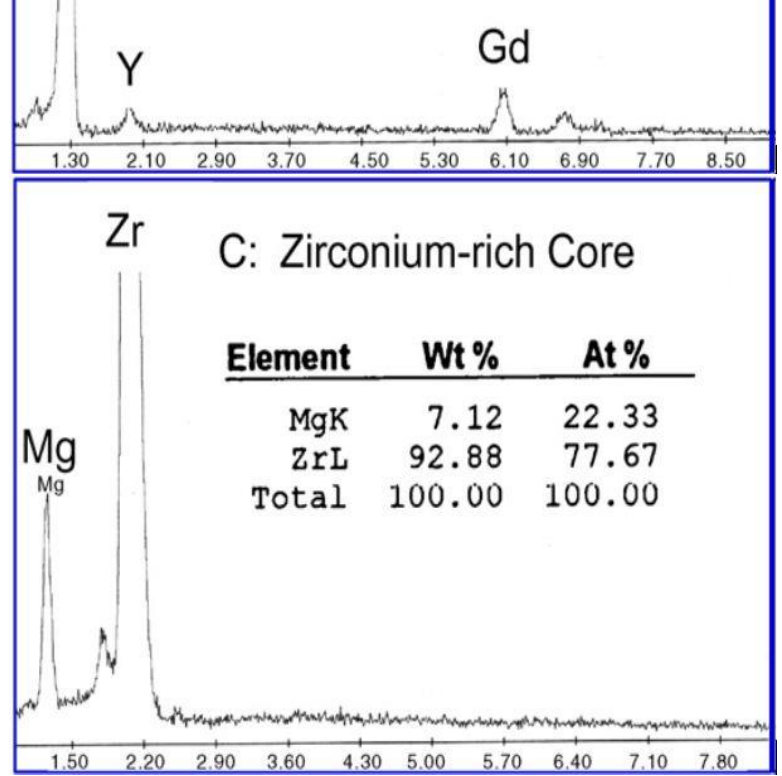

Figure 2.2: SEM image of as-cast sample and the corresponding EDS spectra of the points indicated in the image [57].

\subsubsection{Extruded RE-Mg alloys}

Wrought Mg alloys exhibit superior mechanical properties to casting counterparts due to the hot deformation during extrusion, rolling or forging where these parts are free from pores or cavities and at the same time the mechanical properties are improved through a thermomechanical treatment leading to an ideal microstructure [18,25,26,96-100]. For example, compared with the alloys in the as-cast condition, the microstructures of the alloys 
in the as-extruded condition are greatly refined by dynamic recrystallization (DRX) in the hot extrusion process [78]. Some typical optical micrographs of hot-extruded Mg-8Gd-xZn-0.4Zr $(\mathrm{x}=0,1$, and 3 wt.\%) alloys are shown in Fig. 2.3 [78]. The Gd-containing intermetallic compounds in $\mathrm{Mg}-8 \mathrm{Gd}-\mathrm{xZn}-0.4 \mathrm{Zr}$ alloys were broken during hot extrusion, and then dispersed along the direction of hot extrusion. The average recrystallized grain sizes of $\mathrm{Mg}$ 8Gd-0.4Zr, Mg-8Gd-1Zn-0.4Zr, and Mg-8Gd-3Zn-0.4Zr alloys are about 8, 6, and $3 \mu \mathrm{m}$, respectively. During hot extrusion, recrystallized grains formed on the initially grain boundaries suggest that the accumulation of dislocations at grain boundaries stimulates the DRX process [78], and this also reflects the role of added RE elements [95]. A similar type of extruded microstructures is also reported by Liu et al. [87].

\subsubsection{Heat-treated RE-Mg alloys}

Several studies have been reported regarding the effect of heat treatment on the microstructure of RE-Mg alloys, since these alloys showed a rapid response to precipitation hardening which is attributed to the presence of a high volume fraction of metastable precipitates $[19,57,80,101]$. The superior mechanical properties of RE-Mg alloys are mainly attributed to the metastable and stable precipitates that could remain stable at relatively elevated temperatures $[19,101]$. The effects of heat treatment on the microstructures of $\mathrm{Mg}$ 10Gd-2Y-0.5Zr alloy were presented by He et al. [57] as shown in Fig. 2.4. For the as-cast specimen, the average grain size is $36 \mu \mathrm{m}$. After being solution-treated, the grains coarsen with increasing the solid-solution temperature; the grain size of specimens solution-treated at $470,490,510$, and $530^{\circ} \mathrm{C}$, is $39,43,58$, and $112 \mu \mathrm{m}$, respectively. Similar grain growth has 
been reported for Mg-10Gd-3Y alloy in the T4 and T6 conditions by Dong et al. [102] and Liu et al. [103].

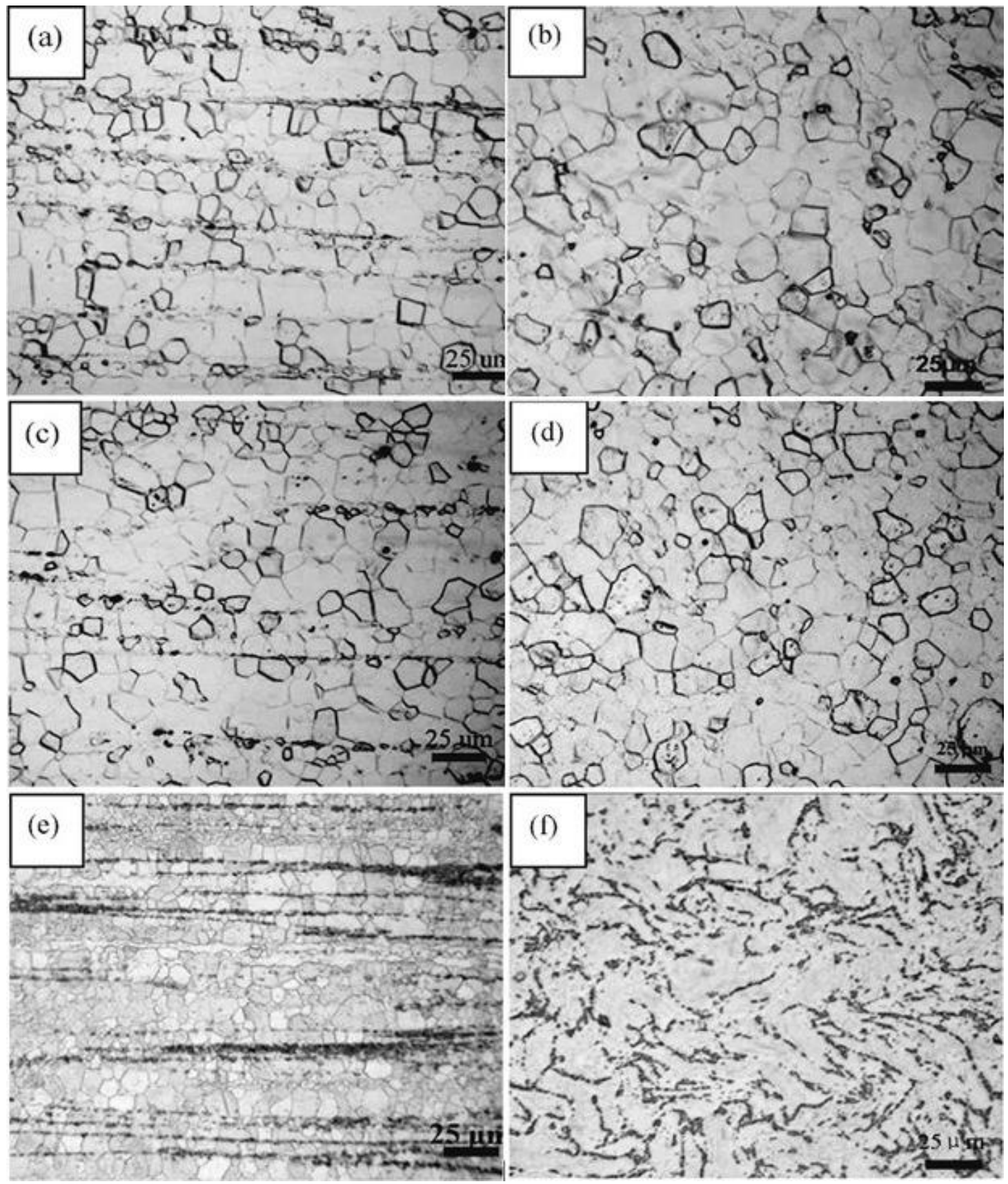

Figure 2.3: Optical images of the as-extruded specimens: (a) and (b) Mg-8Gd-0.4Zr alloy, (c) and (d) Mg-8Gd-1Zn-0.4Zr alloy, and (e) and (f) Mg-8Gd-3Zn-0.4Zr alloy [78]. 

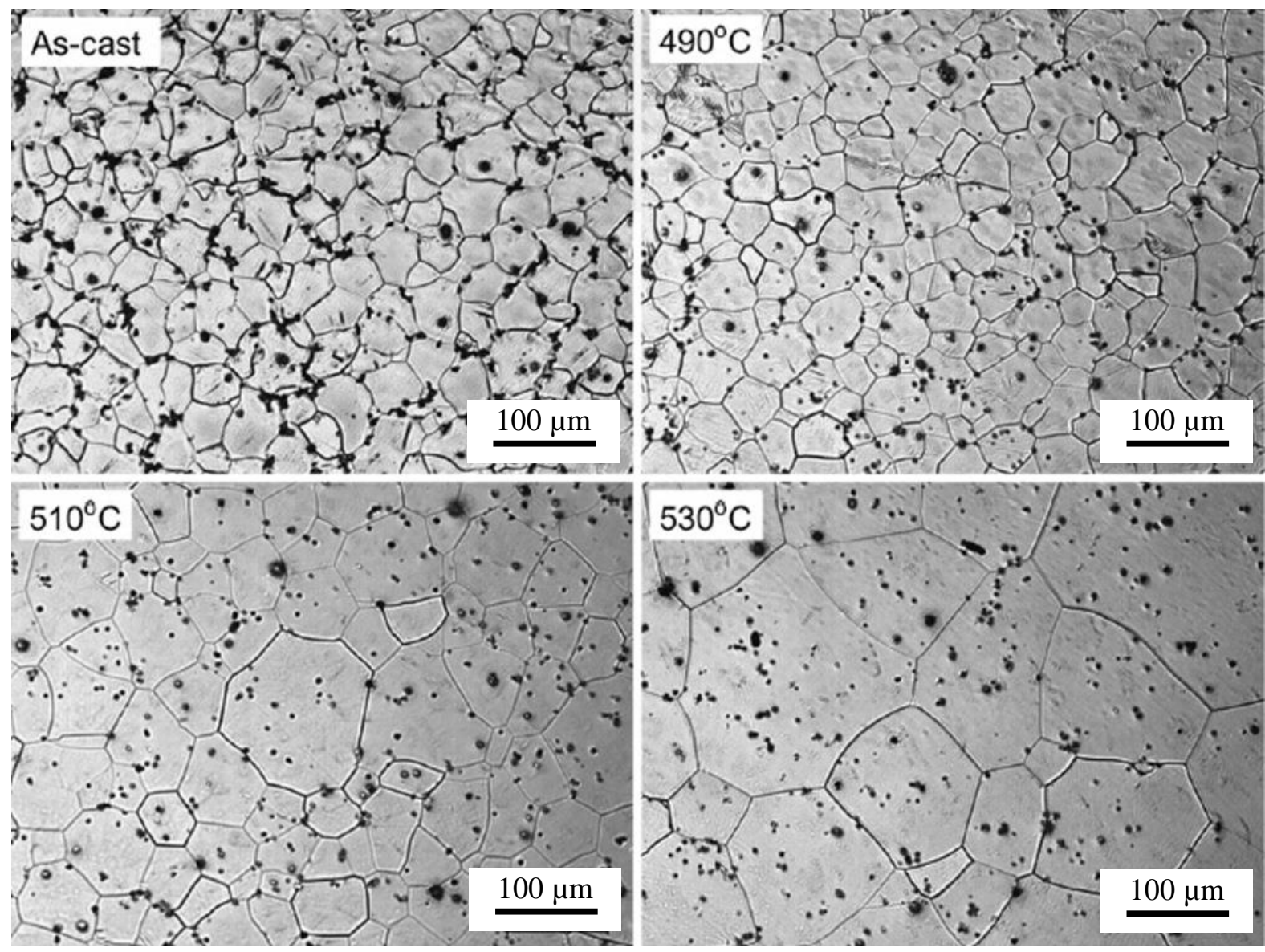

Figure 2.4: Effects of solution treatment temperature on the microstructure of Mg-10Gd-2Y-

$0.5 \mathrm{Zr}$ alloy, whose specimens were solution-treated at the temperature indicated in photographs for 8 h [57].

In addition, Fig. 2.5 shows the X-ray diffraction (XRD) patterns of the GW103 alloy under different conditions [102]. It can be observed that the extruded-F GW103 alloy is mainly composed of $\alpha-\mathrm{Mg}$ solid solution and the $\mathrm{Mg}_{24} \mathrm{Y}_{5}$ secondary phase, where Gd probably substitutes Y. In aging-treated GW103 alloys illustrated in Fig. 2.5(b-d), there are $\mathrm{Mg}_{5}(\mathrm{Gd}, \mathrm{Y})$ precipitates besides $\alpha-\mathrm{Mg}$ and $\mathrm{Mg}_{24} \mathrm{Y}_{5}$ intermetallics, and the $\operatorname{Mg}_{5}(\mathrm{Gd}, \mathrm{Y})$ precipitates increase in number with the aging time. It can also be seen that extruded-T4 is mainly composed of supersaturated $\alpha-\mathrm{Mg}$ solid solution. There are $\mathrm{Mg}_{5}(\mathrm{Gd}, \mathrm{Y})$ precipitates besides 
$\alpha-\mathrm{Mg}$ present in the extruded-T6 alloy. The structure and morphology of the precipitate phases and the precipitation sequence in the RE-Mg alloys have been well established [57,76,104-106]. He et al. [57] investigated the effect of different heat treatment procedures (i.e., aging and solution treatment) on the mechanical behavior of Mg-10Gd-2Y-0.5Zr (wt.\%) alloy. Fig. 2.6 shows some TEM micrographs recorded from a Mg-10Gd-2Y-0.5Zr alloy after aging at $225^{\circ} \mathrm{C}$ for $16 \mathrm{~h}$, with three variants of precipitates formed on $\{11 \overline{2} 0\}_{\alpha}$, and extended along $\langle 1 \overline{1} 00\rangle_{\alpha}$ directions in $[0001]_{\alpha}$ projection. A high number density of precipitates that were almost distributed uniformly in the matrix was observed from Fig. 2.6(a) and (b) and the three-dimensional shape of the precipitates resemble a convex lens elongated along [0001] $]_{\alpha}$ [57]. The precipitates were identified by selected area electron diffractions (SAED), to be $\beta^{\prime}$ a $c$-based centered orthorhombic structure (cbco) phase as Fig. 2.6(c) and (d) [57]. Similar precipitates were also reported in the solution-treated and aged alloys in refs. [76,104,107].

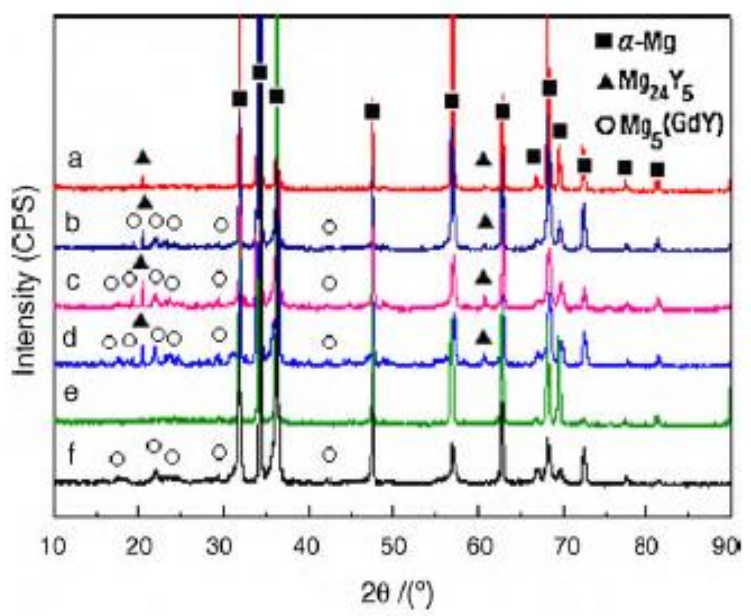

Figure 2.5: XRD analysis of GW103 alloy under different conditions: (a) extruded-F, (b) extruded-UA, (c) extruded-PA, (d) extruded-OA, (e) extruded-T4, and (f) extruded-T6 [102]. 

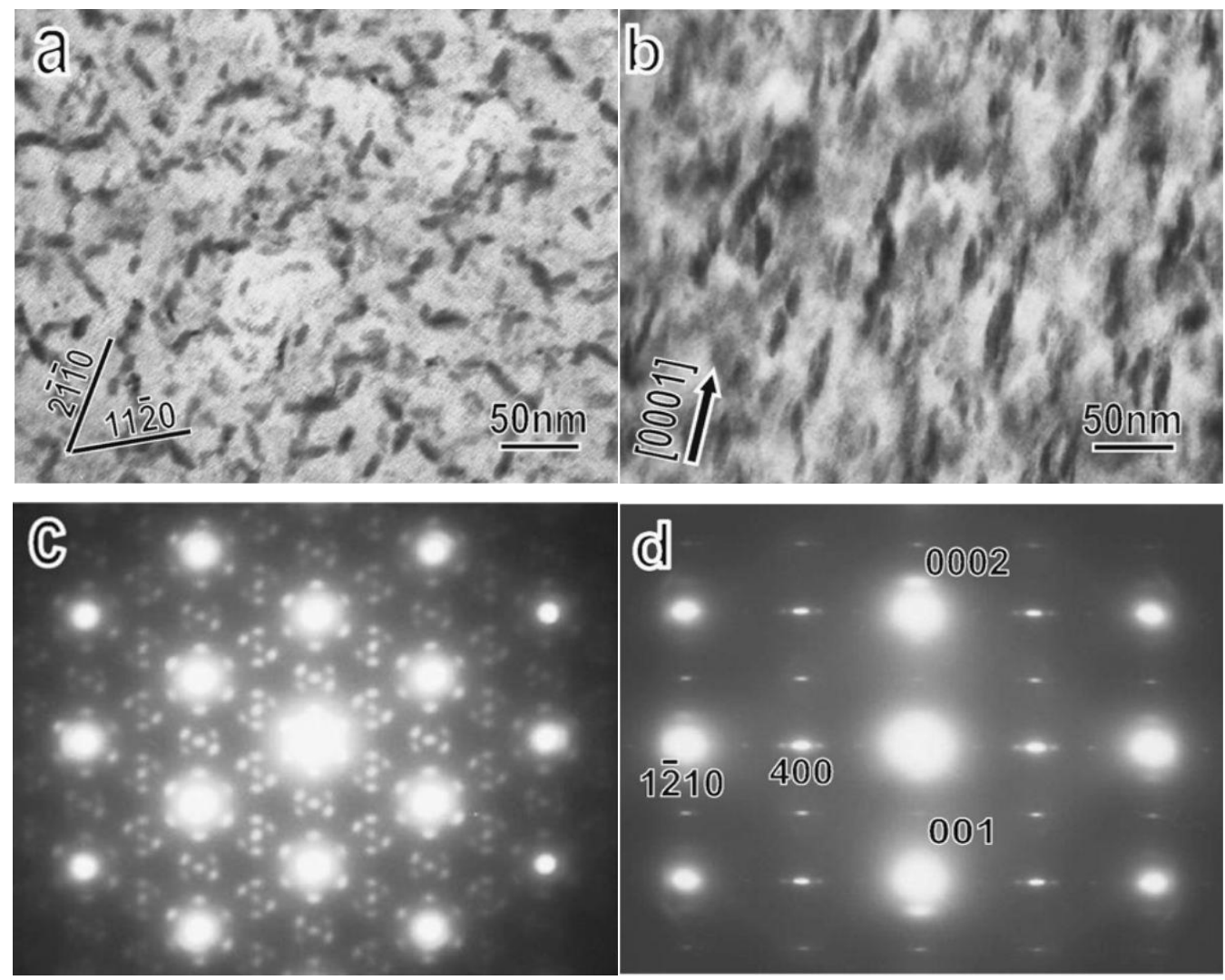

Figure 2.6: Bright field images taken along (a) [0001 $]_{\alpha}$ and $(b)[10 \overline{1} 0]_{\alpha}$ zone axis and corresponding SAED patterns shown in (c) B//[0001 $]_{\alpha}$ and (d) $B / /[10 \overline{1} 0]_{\alpha}$ recorded from a $M g-10 G d-2 Y-0.5 Z$ r alloy aged at $225^{\circ} \mathrm{C}$ for $16 \mathrm{~h}$ [57].

\subsection{Crystallographic textures of RE-Mg alloys}

Wrought $\mathrm{Mg}$ alloys normally exhibit strong basal type textures with basal planes of most grains aligned parallel to the rolling/extrusion direction $[16,36,46,108]$. It has been recently reported that if $\mathrm{Mg}$ is alloyed with $\mathrm{RE}$ elements, the resulting alloy can develop weaker textures and less common than typical textures observed in conventional $\mathrm{Mg}$ alloy sheet during deformation processing [41] or subsequent annealing [109,110]. This provoked 
tremendous attention to a number of RE elements, which are considered effective texture modifiers for Mg alloys, such as Gd [44-47,57-62,75,111-116], Y [45,46,57,58,75,111114,116,117], Ce [46,48,60,116,117], La [47,60,116,117], and $\mathrm{Nd}[46,117-120]$. Even at low alloying levels/concentrations there is a shift in the orientation peak of the extrusion textures in the RE-Mg alloys from $<10 \overline{1} 0>$ to the new positions $<11 \overline{2} 1>,<11 \overline{2} 2>$ or $<20 \overline{2} 1>$ parallel to the extruded direction, which have been referred to as "RE texture components" $[45,46,48,49,59,60,109,116]$. For example, an addition of small amount of Gd $(0.22$ wt.\%) [115] and $\mathrm{Ce}(0.5 \mathrm{wt} \%)$ [52] to $\mathrm{Mg}$ alloys could significantly reduce the texture of the extruded alloy and the addition of $\mathrm{Y}$ element had a similar effect on the texture of $\mathrm{Mg}$ alloys [121,122]. As shown in Fig. 2.7, as the Gd content increased, the texture sharpness decreased in a Mg-Gd binary alloy [115]. The biggest change in texture occurred within the first $\sim 1$ wt.\% addition, after which the change of texture with Gd concentration was less pronounced. Besides, these "RE texture components" are well oriented for basal slip when tested in an appropriate orientation, which results in a substantial gain of ductility and a reduction of the tension-compression asymmetry observed in the conventional wrought $\mathrm{Mg}$ alloys $[25-28,46]$. Bohlen et al. [117] reported that the overall texture intensity and the basal pole intensity aligned with the sheet normal direction were lower for RE-containing alloys than for conventional $\mathrm{Mg}$ alloys, and the typical character of the sheet texture was also altered. It was reported that sheet textures of many $\mathrm{RE}$ elements containing $\mathrm{Mg}$ alloys exhibit a greater tilt of basal poles towards the sheet transverse direction, rather than in the rolling direction (as shown in Fig. 2.8), which promotes more activation of basal slip during loading in the transverse direction than in the rolling direction [123]. 


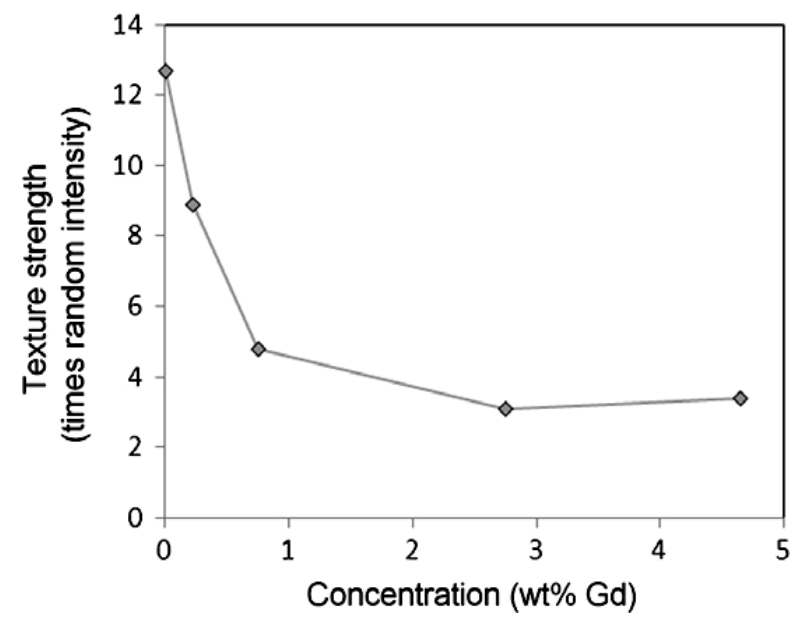

Figure 2.7: Effect of Gd content on the recrystallization texture intensity in a Mg-Gd binary alloy [115].

(a)

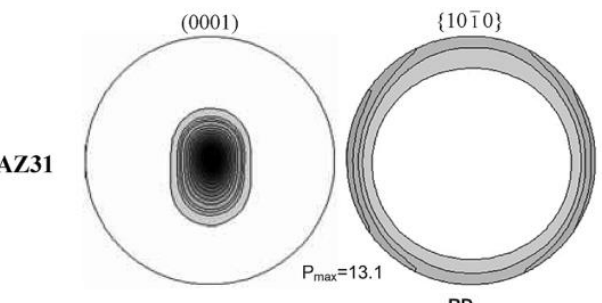

(b)

(c)

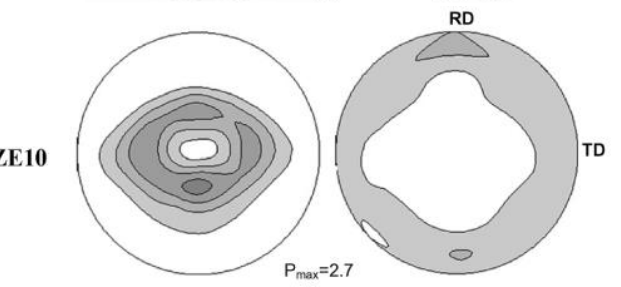

(d)
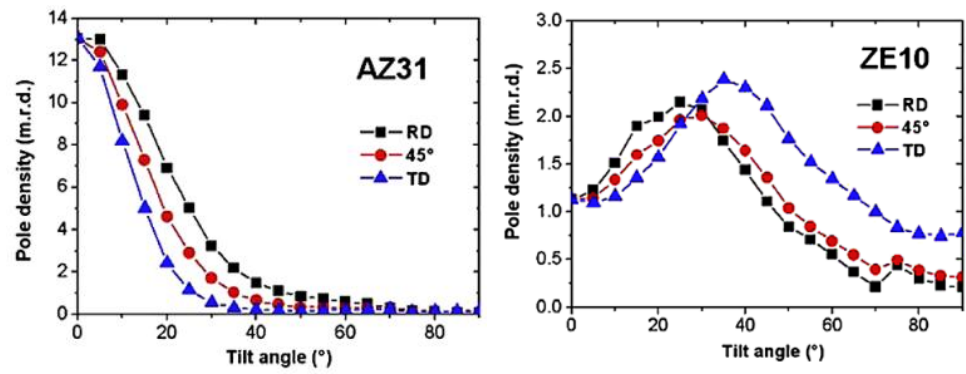

Figure 2.8: (0001) and \{10-10\}pole figures of the as-received (a) AZ31 and (b) ZE10 sheets, where density profiles of the basal pole along different sheet directions are shown for (c) $A Z 31$ and $(d) Z E 10$ sheets (RD: rolling direction, TD: transverse direction, $45^{\circ}: 45^{\circ}$ from the RD) [123]. 


\subsection{Tensile and compressive properties of RE-Mg alloys}

So far, a lot of studies have investigated the mechanical properties of different RE-Mg alloys $[37,41,48,70,78,80-82,113]$. Due to the HCP structure, Mg alloys show high anisotropy in mechanical properties, which is one of the major barriers to the wider application of wrought $\mathrm{Mg}$ alloys. However, it was reported that the addition of RE elements conquers the anisotropy problems by weakening the texture, which could lead to wider applications for RE-Mg alloys [46,47,49]. Experimental results from different investigations on RE-Mg alloys show the dependence of tensile/compressive properties such as yield strength, ultimate strength, elongation and strain hardening exponent on microstructure and crystallographic textures [37,103]. For example, uniaxial tensile and compressive stress-strain curves for a RE-Mg alloy (GW123k) are shown in Fig. 2.9 [37]. It is seen that, compared to the conventional extruded AZ31 Mg alloy, the lack of strong texture in GW123k alloy leads to reduced tension-compression yield asymmetry. Furthermore, the refinement of grains through extrusion, the dispersion of fine precipitated particles and the high content of solute rare-earth elements may provide enough barriers for dislocation slip, thus improving both the compressive and tensile yield strengths. Similar results have been reported in refs. $[41,48,103]$.

\subsection{Fatigue behavior of RE-Mg alloys}

A lot of investigations have been conducted on the fatigue behavior of $\mathrm{Mg}$ alloys in the last

decade or so, included wrought alloys i.e., AZ31 [25-27,124-128], AM30 [28,35], ZK60 
[129,130], and cast alloys, i.e., AZ91 [131-134], AM60 [135-138], AM50 [139-141]. Some information on this aspect could be seen in a recent review [16]. Fatigue properties of RE containing Mg alloys have been studied recently [37,58,86,88-91,102,142-149], which will be discussed in the following sections.
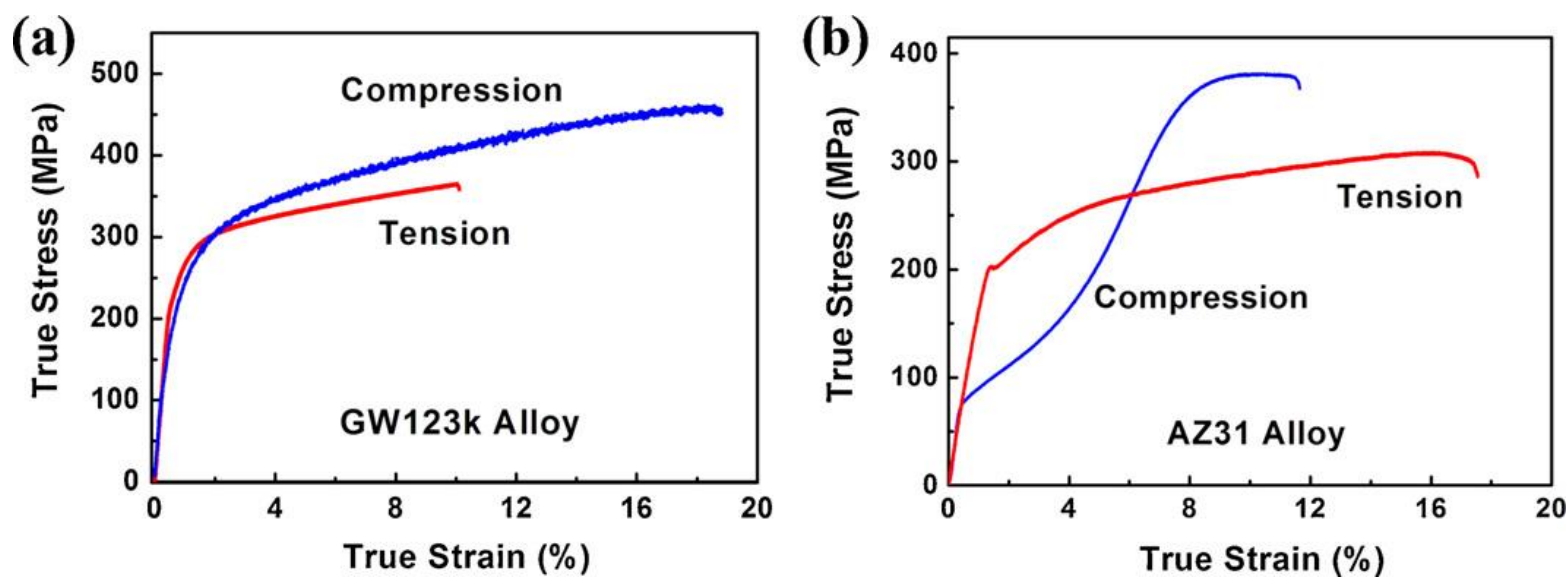

Figure 2.9: Uniaxial tensile and compressive stress-strain curves for (a) GW123k alloy and (b) AZ31 alloy [37].

\subsubsection{Stress-controlled fatigue behavior}

The stress-controlled fatigue data are basically presented by means of stress-life $(S-N)$ curves - a plot of stress $(S)$ (stress amplitude in most cases) against the number of cycles to failure $(N)$ in a $\log$ scale. While the $S-N$ curve approach is old, it represents the most thoroughly examined fatigue characteristics of $\mathrm{Mg}$ and other materials [34,150]. Yang et al. [86,142] investigated the stress-controlled fatigue properties of AZ91D alloy with different concentrations of RE element Ce using the high-frequency fatigue testing machine. Fig. 2.10 shows the typical $S-N$ curves for an AZ91D alloy with varying amounts of Ce addition (1 wt.\% and 2 wt.\%) [86,142]. The addition of 1 wt.\% and $2 \mathrm{wt} . \%$ Ce results in an improvement of 
$20.3 \%$ and $9.1 \%$, respectively, in the fatigue strength of AZ91D alloy. Similar improvements in the high cycle fatigue (HCF) properties were reported in the die-cast AZ91D Mg alloy after adding different amounts $(0.5 \%, 1.0 \%$, and $1.5 \%)$ of $\mathrm{Nd}$ by Yang and $\mathrm{Li}$ [90], in the die-cast AZ91D alloy with or without 1wt.\% Ce by Yang and Liu [89], in the die-cast AZ91D alloys by adding $1 \%$ mischmetal $(50 \% \mathrm{Ce}, 30 \% \mathrm{La}, 12 \% \mathrm{Nd}$, and $6 \% \mathrm{Pr})$ by $\mathrm{Xu}$ et al. [88], and in the gravity cast AZ91 alloy by adding 1\% mischmetal $(67 \% \mathrm{Ce}, 23 \% \mathrm{La}, 7 \% \mathrm{Pr}$ and $2 \% \mathrm{Nd}$ ) by Mokhtarishirazabad et al. [91]. As reported in refs. [86,89,91,142], the factors that influence the HCF properties of die-cast AZ91D alloy include grain size, size and distribution of inclusions, pores and $\mathrm{Mg}_{17} \mathrm{Al}_{12}$ intermetallic particles.

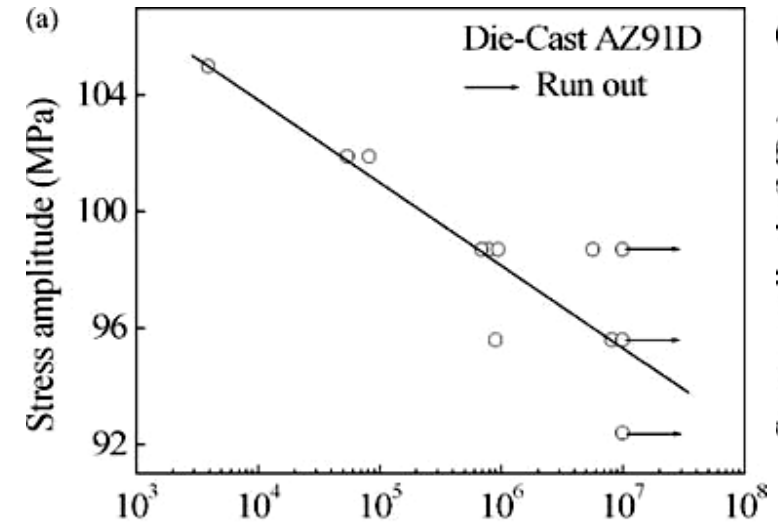

Number of cycles to failure, $N$,

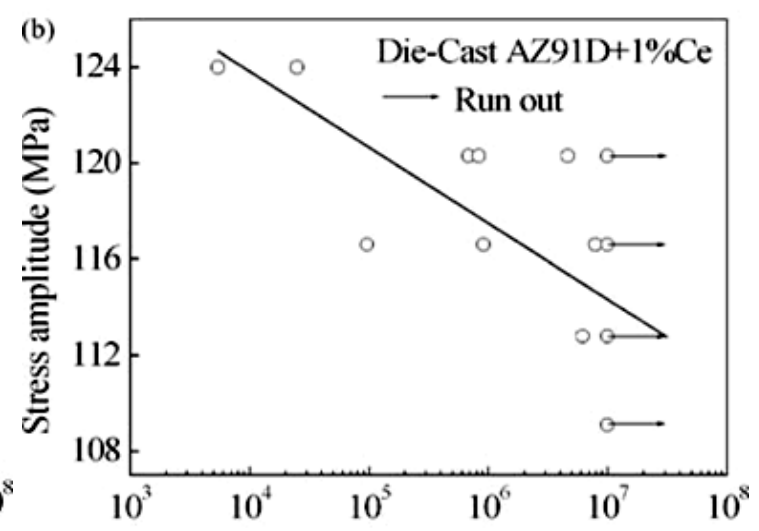

Number of cycles to failure, $N$,

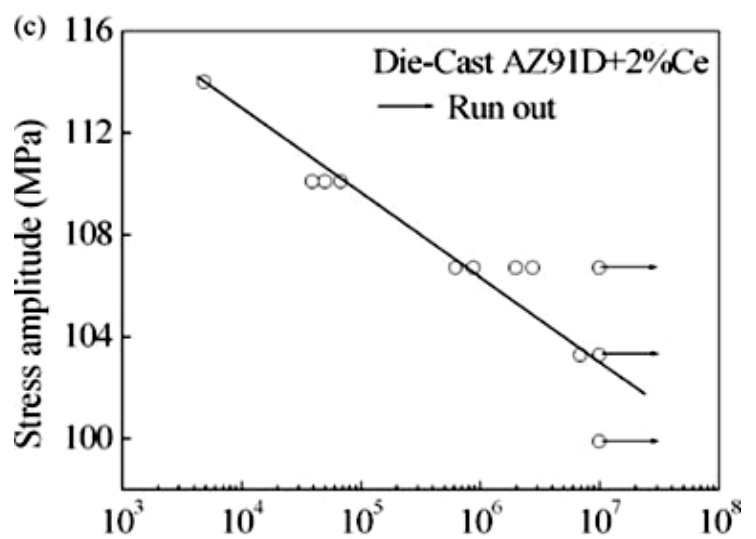

Number of cycles to failure, $N$.

Figure 2.10: $S$ - $N$ curves for an AZ91D alloy with different amounts of Ce addition. (a) 0\% $\mathrm{Ce}$, (b) $1 \% \mathrm{Ce}$, and (c) $2 \% \mathrm{Ce}[86,142]$. 
The grain size was remarkably refined due to the addition of RE elements (e.g., Ce and $\mathrm{Nd}$ ). This led to improved HCF properties of the die-cast AZ91D alloy, since the amount of grain boundaries increases with decreasing grain size, thus increasing the resistance to the movement of dislocations during plastic deformation. As well, the addition of $\mathrm{Ce}$ was observed to result in decreased size and amount of porosity and inclusions (evenly distributed), the fatigue strength of the Mg alloy further increased [89].

Several studies also involved the HCF behavior of Gd and Y containing Mg alloys [37, 102,144,145]. Yang et al. [37] compared the HCF resistance of extruded Mg-12Gd-3Y-0.5Zr (GW123K) alloy with that of conventional extruded AZ31 alloy. The GW123K alloy appears to exhibit a continuous decreasing $S-N$ curve without a horizontal asymptote (as shown in Fig. 2.11) [37] and it has a higher fatigue strength than that of the AZ31 alloy [151]. The addition of $\mathrm{Gd}$ and $\mathrm{Y}$ helped $\mathrm{Mg}$ alloys eliminate the commonly-observed tension-compression yield asymmetry in the conventional wrought $\mathrm{Mg}$ alloys. Similar improvements were also reported in an as-extruded and aged Mg-10Gd-3Y alloy (GW103) by Song et al. [145]. The influence of heat treatment on the fatigue behavior of Mg-10Gd-3Y alloys was investigated by Dong et al. [102], and for all samples tested significant improvements in fatigue life was observed. The fatigue strength in the GW103 alloy was reported to be associated with the threshold stress for basal slip, which can be affected by the solid solution hardening and precipitation hardening effect [102]. As mentioned earlier, the Gd and Y containing precipitates were fairly stable at elevated temperatures, thus enhancing the high temperature fatigue resistance as well [144]. 


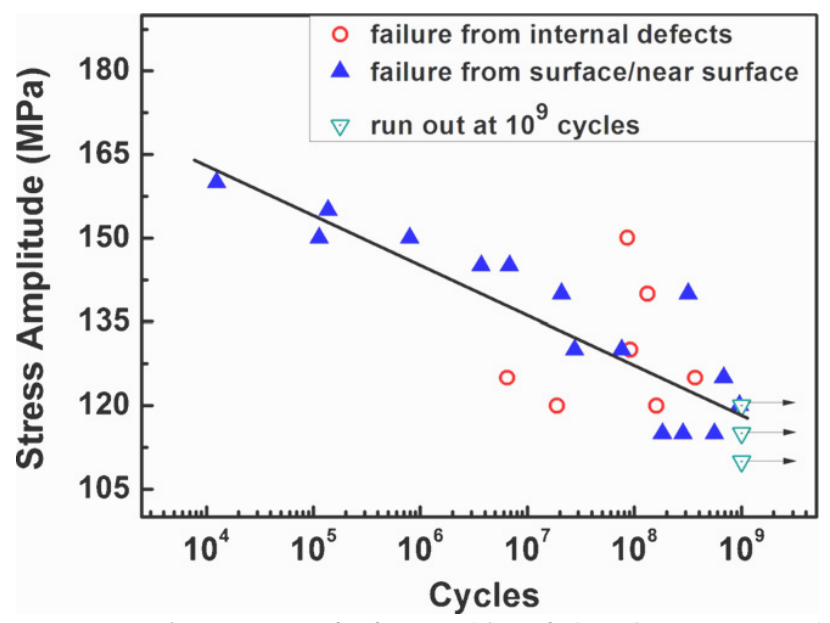

Figure 2.11: S-N curve of an extruded Mg-12Gd-3Y-0.5Zr (GW123K) alloy [37].

\subsubsection{Strain-controlled fatigue behavior}

Strain-controlled low cycle fatigue (LCF) conditions are frequently present where the repeated stresses are of thermal origin and at the notch root $[33,34]$. LCF occurs at the high stress level and low numbers of cycles, and the relevant strain-life data are required in the design of structural components especially in the automotive industry. While there are many reports on the fully reversed $\left(R_{\varepsilon}=-1\right)$ strain-controlled fatigue behavior of RE-free $\mathrm{Mg}$ alloys, e.g., AZ31 [25-27,125,126], ZK60 [129], AM50 [96], AM60B [152,153], and AZ91 $[154,155]$, a number of studies on the strain-controlled LCF behavior of RE-Mg alloys have recently been done [58,147-149]. Most of the studies involved the strain-controlled LCF behavior of Mg-Gd-Y system/alloys. For example, Wang et al. [58] performed the fully reversed strain-controlled tension-compression tests along the extrusion direction of an extruded Mg-8.0Gd-3.0Y-0.5Zr (GW83) alloy with strain amplitudes varying from $0.275 \%$ to $5.0 \%$, and they observed that the GW83 alloy exhibit near-symmetric stress-strain hysteresis loops and marginal cyclic hardening with almost zero mean stress. Other studies 
includes the LCF behavior of extruded Mg-12Gd-3Y-0.5Zr by Yin and Li [148], and extruded Mg-10Gd-2Y-0.5Zr by Zhu et al. [149], and as-cast Mg-10Gd-2.0Y-0.46Zr alloy by Wu et al. [147]. In addition, Fu et al. [146] reported the strain-controlled LCF behavior of AZ91D+0.3\% Ce alloy at room temperature using varying strain amplitudes.

\subsubsection{Stress-strain hysteresis loops}

The analysis of the stress-strain hysteresis loops can give a better understanding on the cyclic deformation behavior of the Mg alloys under LCF condition. Fig. 2.12 shows the stress-strain hysteresis loops of an extruded Mg-8.0Gd-3.0Y-0.5Zr (GW83) alloy obtained from fully reversed strain-controlled fatigue experiment at different total strain amplitudes [58]. Unlike the conventional extruded $\mathrm{Mg}$ alloy where the compression branch of the stress-strain hysteresis loop has a concave-up shape, the lower branch of GW83 has a sigmoidal shape similar to that of the upper branch. With deceasing total strain amplitude, the hysteresis loops become smaller [58]. Fig. 2.13 also illustrates the near-symmetric stress-strain hysteresis loops of an extruded GW123K alloy fatigued at different total strain amplitudes [148]. Similar symmetric hysteresis loops were also reported for AZ91D+0.3\%Ce alloy by Fu et al. [146] and GW102K alloys by Zhu et al. [149].
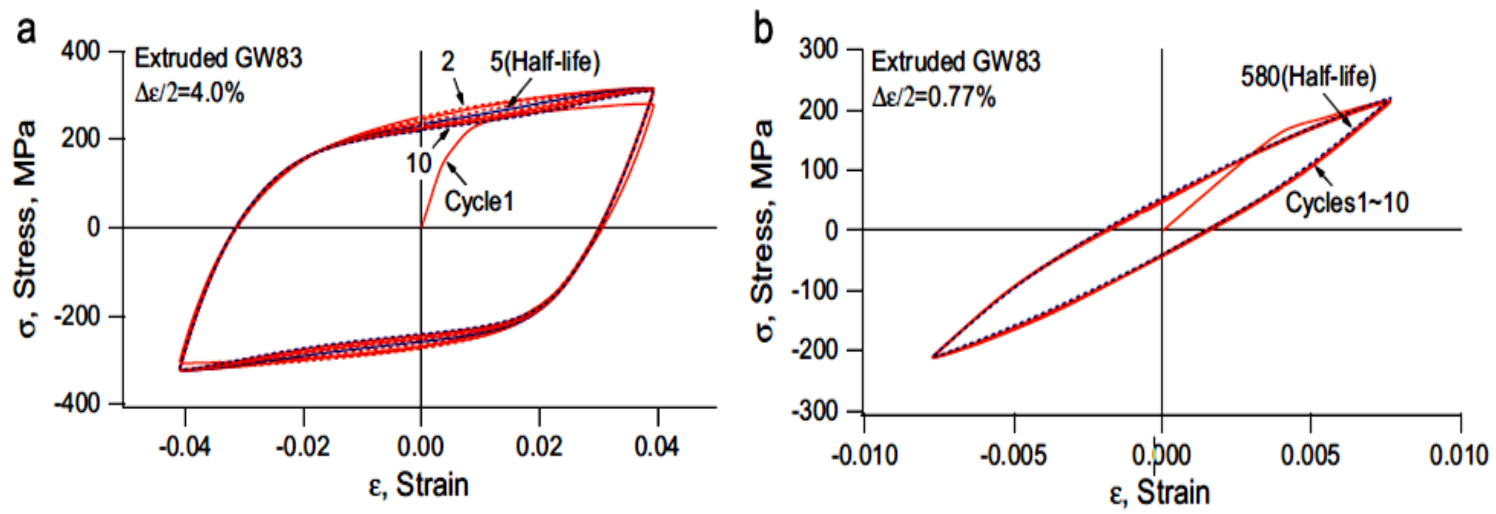


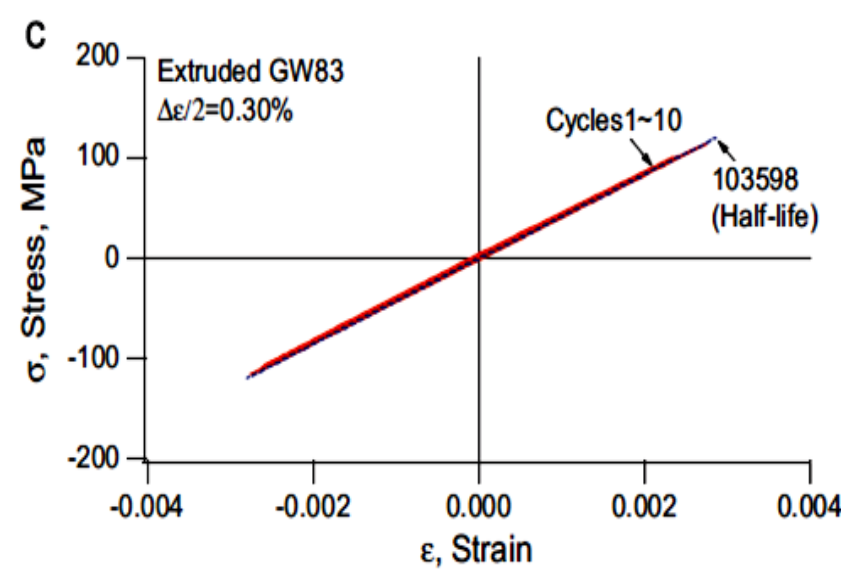

Figure 2.12: Stress-strain hysteresis loops of an extruded GW83 alloy fatigued at (a) $\Delta \varepsilon / 2=4.0 \%$, (b) $\Delta \varepsilon / 2=0.77 \%$, and (c) $\Delta \varepsilon / 2=0.30 \%[58]$.

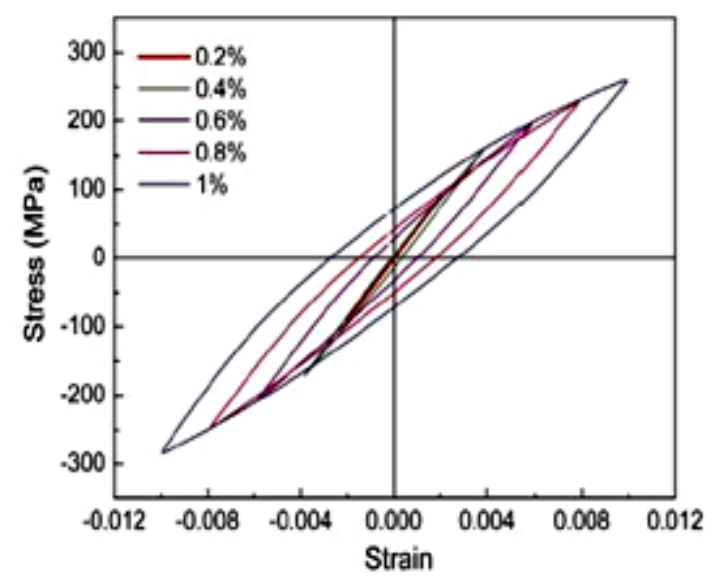

Figure 2.13: Hysteresis loops of a GW123K alloy fatigued at varying total strain amplitudes [148].

\subsubsection{Cyclic deformation response}

In the total strain controlled fatigue tests the cyclic stress response of a material during cyclic deformation represents the change of the stress amplitude with consecutive cycles, and illustrates the path by which the material achieves its final stress level. Fig. 2.14 shows the evolution of stress amplitudes with respect to the number of cycles at different applied strain 
amplitudes in an extruded Mg-8.0Gd-3.0Y-0.5Zr (GW83) alloy [58]. As the total strain amplitude increased, the stress amplitude increased and the fatigue life of the material decreased. When the strain amplitude is below $1.0 \%$, the material displays nearly stable or slightly cyclic hardening behavior that may be attributed to the dislocation transformation effects by the increased dislocation slips [156]. On the other hand, when the strain amplitude is higher than $1.0 \%$, the alloy exhibits first cyclic hardening and then cyclic softening until fracture. Other studies also showed that the cyclic stress response of the RE-containing $\mathrm{Mg}$ alloys is rather stable, especially at a low strain amplitude of $0.2 \%$ for an extruded GW123K alloy [148].

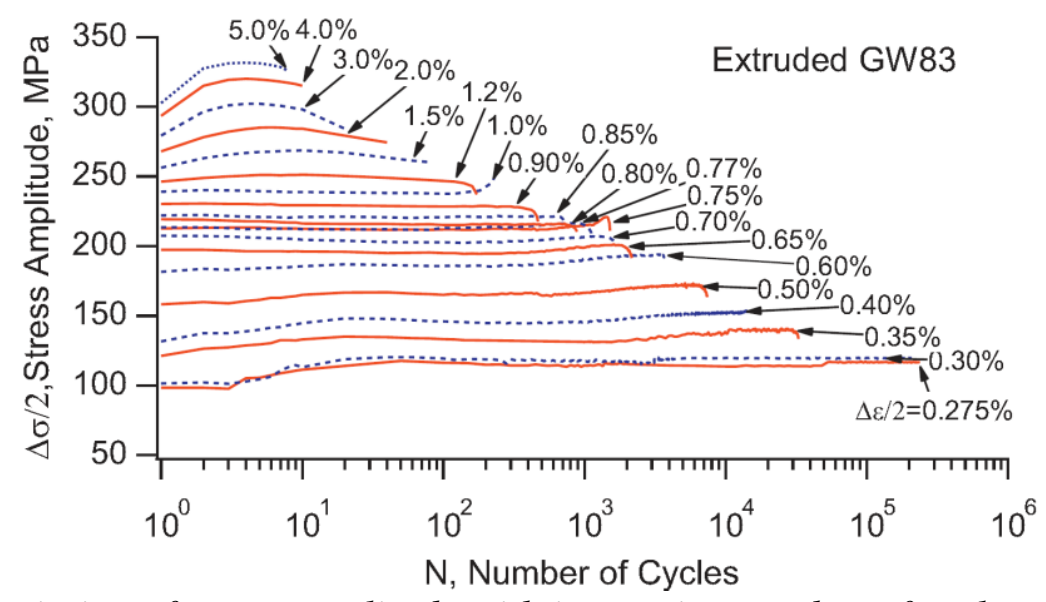

Figure 2.14: Variation of stress amplitude with increasing number of cycles at varying strain amplitudes from $0.275 \%$ to $5.0 \%$ for an extruded GW83 alloys [58].

\subsubsection{Fatigue life and parameters}

The RE-Mg alloys exhibit a longer fatigue life as compared with RE-free wrought Mg alloys [26,28,148,149,156-159]. Zhu et al. [149] reported that the Mg-10Gd-2Y-0.5Zr (GW102K) alloy showed a superior fatigue resistance as compared to the AZ31 alloy at the same total 
strain amplitude, as shown in Fig. 2.15. Similarly, Yin and Li [148] compared the fatigue life of GW123K alloy with some RE-free wrought Mg alloys, e.g., AM60 [160], AZ31 [26], and Al-Mg-Sc alloy [161], and observed that the GW123K alloy exhibited decent low-cycle fatigue properties, where the relation between the strain amplitude and fatigue life can be described by Coffin-Manson and Basquin relations. Fu et al. [146] reported that the fatigue life of AZ91D+Ce alloy could be described by the Manson-Coffin law as well. It should be noted that a distinct kink point was observed in the fatigue curve of a RE-Mg alloy (GW83) [58], although some earlier studies also showed such a distinct kink point in the strain-life fatigue curves of extruded AZ31B and ZK60 Mg alloys [158,162].

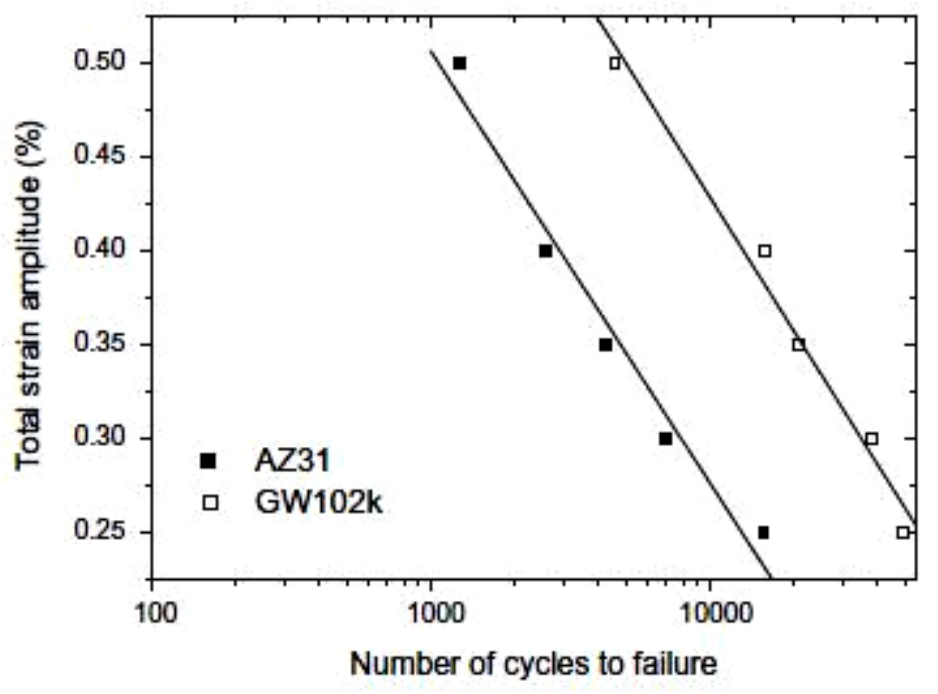

Figure 2.15: Total strain amplitude-fatigue life curves for GW102K and AZ31 alloys [149].

\subsection{Summary}

As a general consensus, the addition of RE elements can reduce the so-called unfavorable mechanical anisotropy and unwanted tension-compression yield asymmetry of $\mathrm{Mg}$ alloys. The microstructures, crystallographic textures, and tensile and compressive properties in 
relation to the addition of RE elements have been previously reported. However, since RE$\mathrm{Mg}$ alloys are relatively new, very few data (mainly the stress-controlled fatigue) on the fatigue behavior of such Mg alloys have been reported in the literature. Thus, it is still far from our understanding on the cyclic deformation mechanisms related to the strain-controlled low cycle fatigue tests. Significant research efforts to understand the cyclic deformation behavior, stress response, and develop life prediction methodologies for the RE-Mg alloys are required. 


\section{CHAPTER 3}

\section{EXPERIMENTAL PROCEDURE}

\subsection{Test Materials}

Two rare-earth containing Mg alloys, GW103K and NZ30K alloys in the form of extruded bars for the current investigation were received from "The State Key Laboratory of Metal Matrix Composites”, School of Materials Science and Engineering, Shanghai Jiao Tong University, Shanghai, PR China. The chemical composition of both alloys is listed in Table 3.1. The extrusion was performed at a die temperature of $400^{\circ} \mathrm{C}$ via induction heating without holding (since the final extrusion temperature of $400^{\circ} \mathrm{C}$ could be reached in a few minutes) with an extrusion ratio of 9:1 at an extrusion ram speed of $3 \mathrm{~mm} / \mathrm{s}$ and a die angle of $60^{\circ}$. The entire process of heating and extrusion was completed within about 10 minutes and the extruded bars were then water quenched. For the sake of comparison, some compression and fatigue samples of RE-free extruded AM30 Mg alloy (with a composition of 3.4 wt.\% Al, 0.33 wt.\% Mn, 0.16 wt.\% Zn, 0.0026 wt.\% Fe, 0.0006 wt.\% Ni, 0.0008 wt.\% $\mathrm{Cu}$ and balance $\mathrm{Mg}$ ) were also tested.

Table 3.1: Chemical composition (in wt.\%) of GW103K and NZ30K alloys.

\begin{tabular}{ccccccc}
\hline Alloy & Gd & Nd & Y & $\mathrm{Zn}$ & $\mathrm{Zr}$ & $\mathrm{Mg}$ \\
\hline GW103K & 10 & - & 2.7 & - & 0.4 & Balance \\
NZ30K & - & 3 & - & 0.2 & 0.5 & Balance \\
\hline
\end{tabular}




\subsection{Heat treatment}

After extrusion the extruded bars were subjected to two types of heat treatment: (1) direct peak aging at $225^{\circ} \mathrm{C}(\mathrm{GW} 103 \mathrm{~K})$ and $200^{\circ} \mathrm{C}(\mathrm{NZ30K})$ for $16 \mathrm{~h}$ (T5 heat treatment), and (2) solution at $500^{\circ} \mathrm{C}(\mathrm{GW} 103 \mathrm{~K})$ and $520^{\circ} \mathrm{C}(\mathrm{NZ30K})$ for $2 \mathrm{~h}$, then water cooling, followed by isothermally aging at $225^{\circ} \mathrm{C}(\mathrm{GW} 103 \mathrm{~K})$ and $200^{\circ} \mathrm{C}(\mathrm{NZ30K})$ for $16 \mathrm{~h}$ in an oil bath furnace (T6 heat treatment as shown in Fig. 3.1). These extruded, T5, and T6 heat treated bars were subsequently machined for tensile, compressive, and fatigue tests with the loading axis parallel to the extrusion direction (ED).

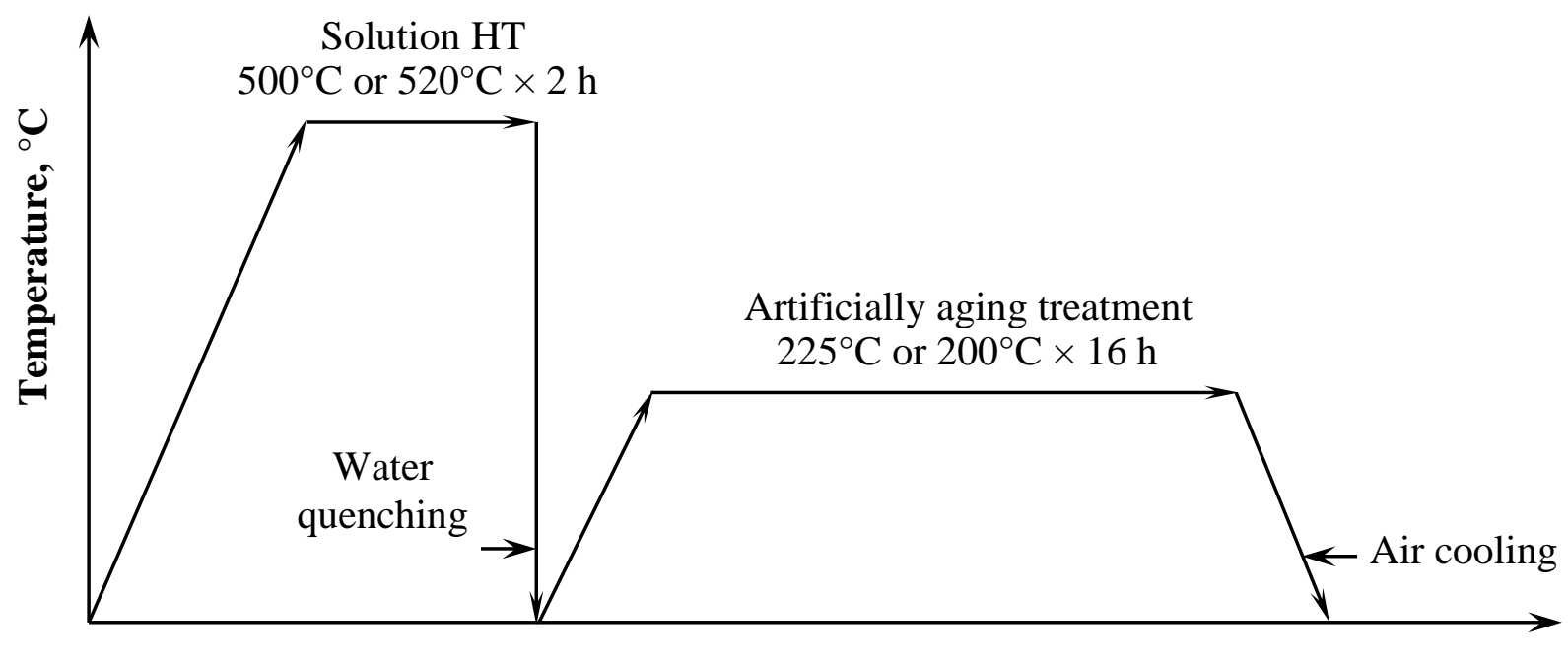

Time, $\mathbf{h}$

Figure 3.1: T6 heat treatment procedure for the RE-Mg alloys. 


\subsection{Sample preparation and testing}

The metallographic and mechanical test samples are prepared according to the following procedures.

\subsubsection{Metallography}

All metallographic samples (approx. $5 \mathrm{~mm} \times 5 \mathrm{~mm}$ in the cross section) for the microstructural characterization were cut from both GW103K and NZ30K alloy bars. The samples were cold mounted using LECO 7007 resin powder and liquid (mixing ratio: 2 parts of resin added to 1 part of catalyst). Hot mounting was avoided to prevent any possible microstructural change due to the effect of temperature during sample preparation. The mounted samples were manually ground with $\mathrm{SiC}$ sand papers with a grit number of 320, 600, and 1200, respectively. Water was used as lubricant in each grinding step. Polishing was

carried out with $6 \mu \mathrm{m}, 3 \mu \mathrm{m}$, and $1 \mu \mathrm{m}$ diamond paste using diamond extender (a mixture of rust inhibiting solution with distilled water $-10 \%$ solution by volume) as lubricant. Cleaning of the mount after polishing involved dipping in and spraying ethanol, ultrasonically cleaning in ethanol, followed by drying with compressed air. After the final polishing with $0.05 \mu \mathrm{m}$ alumina paste (master prep), the polished samples were etched with Acetic-Picral (10 ml acetic acid, $4.2 \mathrm{~g}$ picric acid, $10 \mathrm{ml}$ distilled water, and $70 \mathrm{ml}$ ethanol) for GW103K alloys and with Vilella's reagent $(1 \mathrm{~g}$ picric acid, $5 \mathrm{ml} \mathrm{HCl}$, and $100 \mathrm{ml}$ ethanol) for $\mathrm{NZ30K}$ alloys to reveal the macroscopic structure (grain boundaries) of these alloys. 


\subsubsection{Quantitative image analysis}

Microstructural examinations were performed using an optical microscope (OM) equipped with Clemex quantitative image analysis software, scanning electron microscope (SEM) JSM-6380LV equipped with Oxford energy dispersive X-ray spectroscopy (EDS) system and three-dimensional (3D) surface/fractographic analysis capacity, and transmission electron microscope (TEM) JEM-2100 (location: Shanghai Jiao Tong University, Shanghai, PR China) operated at $200 \mathrm{kV}$. The average grain size was measured via linear intercept method. Overall at least 15 locations for each sample were examined and the average values will be reported in this report. The Clemex image analysis system was comprised of a Clemex CMT software adaptable to ASTM standards, a Nikon optical microscope (10x eye piece, five different object lenses with magnifications of $5 \times, 10 \times, 20 \times, 40 \times$, and 100x), a highresolution digital camera, and a high performance computer to carry out the detailed analysis.

\subsubsection{Phase identification by $X$-ray diffraction}

Following the SEM analysis, a further verification of the phases formed was done via X-ray diffraction analysis. For this, a multi-functional PANalytical X'Pert PRO X-ray diffractometer was used to identify the formation of phases in the samples. XRD was performed using $\mathrm{Cu} K_{\alpha}$ radiation (wave length $\lambda=0.15406 \mathrm{~nm}$ ) at $45 \mathrm{kV}$ and $40 \mathrm{~mA}$. The diffraction angle $(2 \theta)$ at which the X-rays hit the sample varied from $40^{\circ}$ to $100^{\circ}$ with a step size of $0.04^{\circ}$ and $2 \mathrm{~s}$ in each step. 


\subsubsection{Texture measurements by $X$-ray diffraction}

Texture measurements were performed on the as-received samples of both GW103K and NZ30K alloys. The polishing procedure was the same as the one employed for microstructural observations and image analysis but without etching. The texture was determined by measuring incomplete pole figures between $\Psi=0$ to $75^{\circ}$ in the back reflection mode using a PANalytical X'Pert PRO X-ray diffractometer with $\mathrm{Cu} K_{\alpha}$ radiation at $45 \mathrm{kV}$ and $40 \mathrm{~mA}$. The texture measurements were designed to determine the intensity variation of a certain diffraction peak, indexed $\boldsymbol{h}=(h k l)$, as a function of the measurement direction $(\boldsymbol{y})$ relative to the sample-reference frame. After corrections and normalizations, the probability maps, $P(\boldsymbol{h}, \boldsymbol{y})$, or pole figures were constructed to describe the distribution of different crystal directions in the sample space using MTEX software [163]. A set of five pole figures ( $\{10 i 0\}$, $\{0002\},\{1011\},\{11 \overline{2} 0\},\{10 \overline{1}\}\})$ were used to calculate the orientation distribution function (ODF) and to represent ODF, Bunge notations of the Euler angles $\left(\begin{array}{lll}\varphi_{1} & \Phi & \varphi_{2}\end{array}\right)$ were implemented throughout. Defocusing due to the rotation of XRD sample holder was corrected using experimentally determined data obtained from the diffraction of Mg powders received from Magnesium Elektron.

\subsubsection{Tensile tests}

The sub-sized tensile samples in accordance with ASTM E8 standard [164] were machined with the loading axis parallel to the extrusion direction (ED). The sample geometry and dimensions are shown in Fig. 3.2. The samples had a gauge length of $25 \mathrm{~mm}$ (or a parallel 
length of $32 \mathrm{~mm}$ ) and a width of $6 \mathrm{~mm}$. The thickness of the samples was $6 \mathrm{~mm}$ as well. The specimens were hand-ground progressively along the loading direction with $1 / 0,2 / 0,3 / 0,4 / 0$ emery papers to remove the machining marks and to achieve a smooth surface. The gage section of fatigue samples was ground progressively along the loading direction with emery papers up to a grit number of 600 to remove the machining marks and to achieve a consistent surface. The tests were carried out using a computerized United tensile testing machine at a strain rate of $1 \times 10^{-4} \mathrm{~s}^{-1}$ for both $\mathrm{GW} 103 \mathrm{~K}$ and NZ30K Mg alloys at room temperature. At least two samples were tested to ensure the reproducibility. The strain was measured using an extensometer with a gauge length of $25 \mathrm{~mm}$.

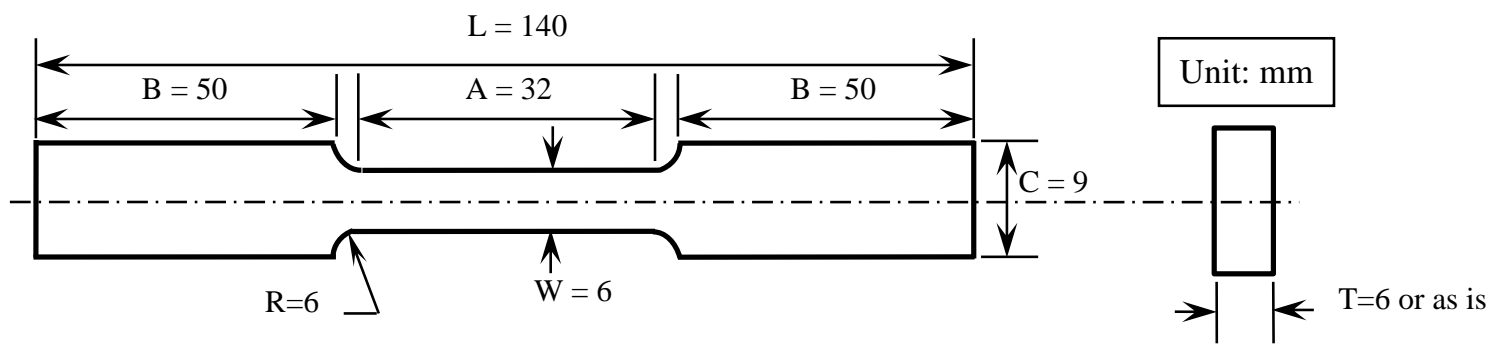

Figure 3.2: Geometry and dimensions of the sub-sized tensile/fatigue test specimens according to ASTM E8 [164].

The tensile properties, including $0.2 \%$ yield strength (YS), ultimate tensile strength (UTS) and percent elongation to failure $(\% \mathrm{El})$ were evaluated from the engineering stress-strain curves. The strain (or work) hardening exponent $(n)$ and the strength coefficient $(K)$ were determined using the Hollomon equation $\left(\sigma=K \varepsilon^{n}\right)$ by plotting $\log \sigma$ vs. $\log \varepsilon$. 


\subsubsection{Compression tests}

The samples for compression tests were machined from the as-received bars of GW103K alloy according to the ASTM E9M standard [165]. All samples were machined with the compression axis parallel to the extrusion direction (ED) using a center lathe machine turned down to cylindrical samples. A schematic of the samples is shown in Fig. 3.3. Compression tests were carried out for GW103K Mg alloy using a computerized Instron machine at room temperature at a strain rate between $1 \times 10^{-1} \mathrm{~s}^{-1}$ and $1 \times 10^{-4} \mathrm{~s}^{-1}$. The deformed samples were cut along the compression axis diameter using a slow diamond cutter, cold-mounted, ground, polished, and etched to examine the evolution of microstructure during compression. It should be noted that in evaluating the stress-strain curves and strain hardening rates, the machine deformation was eliminated using a calibration curve to obtain the actual or net deformation of test samples.

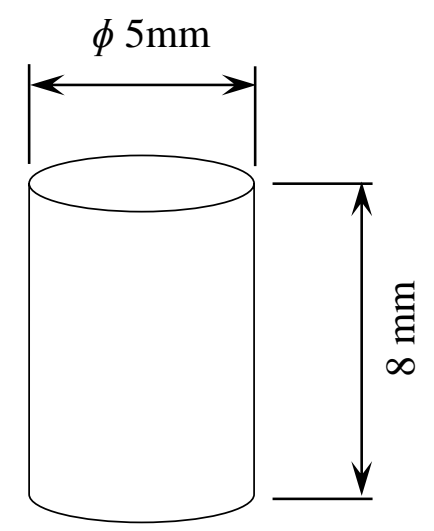

Figure 3.3: Specimen dimension for the compression tests, prepared according to ASTM E 9M standards [165]. 


\subsubsection{Volume fraction of twins}

In order to calculate the volume fractions of twins of the deformed samples from the compression tests a simple point counting procedure according to ASTM E562-11 standard [166] was used. The cutting and polishing procedure of the samples was the same as the one employed for microstructure analysis to examine the evolution of deformation twins during compression. Five digital micrographs were taken from each sample at a magnification of $200 \times$, and then a grid of 31 points by 39 points was superimposed on the images. The volume fraction was determined as a ratio of the number of points positioned within the twins to the total number of grid points.

\subsubsection{Fatigue tests}

Strain-controlled, pull-push type fatigue tests were conducted using a computerized servohydraulic Instron 8801 fatigue testing system via a Fast Track Low Cycle Fatigue (LCF) program at a constant strain rate of $1 \times 10^{-2} \mathrm{~s}^{-1}$ and room temperature of $25^{\circ} \mathrm{C}$. The tests were carried out in a strain control mode according to ASTM E606 standard [167]. Triangular strain waveform was applied during the tests. Low cycle fatigue tests were performed at zero mean strain $\left(R_{\varepsilon}=-1\right.$, completely reversed strain cycle), at total strain amplitudes of $0.2 \%$, $0.4 \%, 0.6 \%, 0.8 \%, 1.0 \%$, and $1.2 \%$, and at least two samples were tested at each level of the strain amplitudes. The calibration of both load and strain channels was performed for each individual sample prior to testing. The strain-controlled tests at lower strain amplitude levels were continued up to 10,000 cycles, then the tests were changed to load control at a 
frequency of $50 \mathrm{~Hz}$ using sine waveform. The fracture surfaces of fatigued specimens were examined via scanning electron microscope (SEM) to identify fatigue crack initiation sites and propagation characteristics. The residual twins in the region near the fracture surface were observed as well.

To study the effect of strain ratio on the low cycle fatigue behavior of the as-extruded GW103K alloy, five different strain ratios, i.e., $R_{\varepsilon}=0.5,0,-1,-3$, and $-\infty$, were used at a given total strain amplitude of $0.8 \%$ and at least two samples were tested at each level of the strain ratio. The varying strain ratio tests were performed in the same manner at a strain rate of $1 \times 10^{-2} \mathrm{~s}^{-1}$ and room temperature with triangular waveform loading as well. The test parameters used in the low cycle fatigue tests under different strain ratios at a constant amplitude of $0.8 \%$ and a strain rate $1 \times 10^{-2} \mathrm{~s}^{-1}$ are listed in Table 3.2. After fatigue tests, the SEM equipped with an energy dispersive X-ray spectroscopy (EDS) system was used to examine the fatigue crack initiation sites and identify the mechanism of fatigue crack propagation under the above applied conditions.

Table 3.2: Test parameters under different strain ratios at a strain amplitude of $0.8 \%$ and a strain rate of $1 \times 10^{-2} \mathrm{~s}^{-1}$.

\begin{tabular}{cccc}
\hline $\begin{array}{c}\text { Strain ratio } \\
\left(R_{\varepsilon}\right)\end{array}$ & $\begin{array}{c}\text { Mean strain } \\
\left(\varepsilon_{\text {mean }}\right), \%\end{array}$ & $\begin{array}{c}\text { Maximum strain } \\
\left(\varepsilon_{\text {max }}\right), \%\end{array}$ & $\begin{array}{c}\text { Minimum strain } \\
\left(\varepsilon_{\text {min }}\right), \%\end{array}$ \\
\hline 0.5 & 2.4 & 3.2 & 1.6 \\
0 & 0.8 & 1.6 & 0 \\
-1 & 0 & 0.8 & -0.8 \\
-3 & -0.4 & 0.4 & -1.2 \\
$-\infty$ & -0.8 & 0 & -1.6 \\
\hline
\end{tabular}




\section{CHAPTER 4}

\section{DEFORMATION BEHAVIOR OF AN EXTRUDED GW103K ALLOY DURING COMPRESSION}

In contrast to the cubic metals, the deformation of $\mathrm{Mg}$ is fairly complex because its hexagonal close-packed (HCP) crystal structure has a limited number of active slip systems at room temperature, with basal slip and twinning playing a key role in the plasticity of $\mathrm{Mg}$ alloys [25,27]. Both basal slip and twinning mechanisms tend to align the $c$-axes of grains with the direction of predominantly compressive strains during forming (rolling, extrusion). In the $\mathrm{Mg}$ sheet and plate, the $c$-axes align with their normal direction; in the extrusion the $c$ axes tend to align transversely to the extrusion axis. These forming processes would result in the presence of a strong preferred orientation or texture in $\mathrm{Mg}$ alloys.

As mentioned earlier in section 1.1, it has recently been observed that Mg alloys containing RE elements can develop more random textures during hot extrusion [59]. The addition of $\mathrm{RE}$ elements in $\mathrm{Mg}$ alloys leads to a decrease of the overall texture sharpness allowing the activation of easy basal slip to a higher extent, compared to other RE-free wrought Mg alloys $[59,60,61]$. Furthermore, RE elements could result in the formation of a $\{1 \overline{2} 11\}$ texture component parallel to the extrusion direction $[59,60,61]$. In the presence of strong texture, wrought $\mathrm{Mg}$ alloys were prone to over-concentrated twinning-detwinning activities which may aggravate the cyclic deformation irreversibility and increase the propensity of crack initiation [25,27]. During cyclic deformation, the level of strain amplitudes applied at a strain ratio of $R=-1$ was normally limited to a maximum value of about $1 \sim 2 \%$ in both tensile and 
compressive phases [25]. This would potentially limit the occurrence of twinning to its full capacity in the compressive phase during cyclic deformation. Although the addition of RE elements to $\mathrm{Mg}$ alloys offers the possibility of effectively improved mechanical properties via texture weakening, it remains unclear what role the RE elements play in the formation of twins and how the twinning develops beyond the above specific strain level, whether the detwinning would occur with increasing compressive strain, and how the twinning or detwinning affects the strain hardening behavior. This part of the dissertation was aimed to identify the deformation behavior of an extruded GW103K alloy in compression along the extrusion direction at different strain levels, with special attention to the effect of the RE element addition on the twin formation.

\subsection{Microstructure and texture}

Fig. 4.1(a) shows a typical optical image on the microstructure of GW103K alloy in the asextruded condition where the ED is indicated. For the sake of comparison, the microstructure of an extruded AM30 Mg alloy is shown in Fig. 4.1(b) as well. It is seen that uniform equiaxed grains with an average grain size of about $12 \mu \mathrm{m}$ were obtained in the GW103K alloy (Fig. 4.1(a)) due to the occurrence of dynamic recrystallization (DRX) in the hot extrusion process at $400^{\circ} \mathrm{C}$, which is fairly small in comparison with AM30 alloy (Fig. 4.1(b)). This was due to the role of added RE elements and zirconium ( $\mathrm{Zr}$ ) where $\mathrm{Zr}$ mainly restricted the grain growth [95]. A similar role of grain refinement by RE elements, e.g., gadolinium [168], yttrium [169], cerium [170], neodymium [44,171], erbium [172], has been reported as well. 

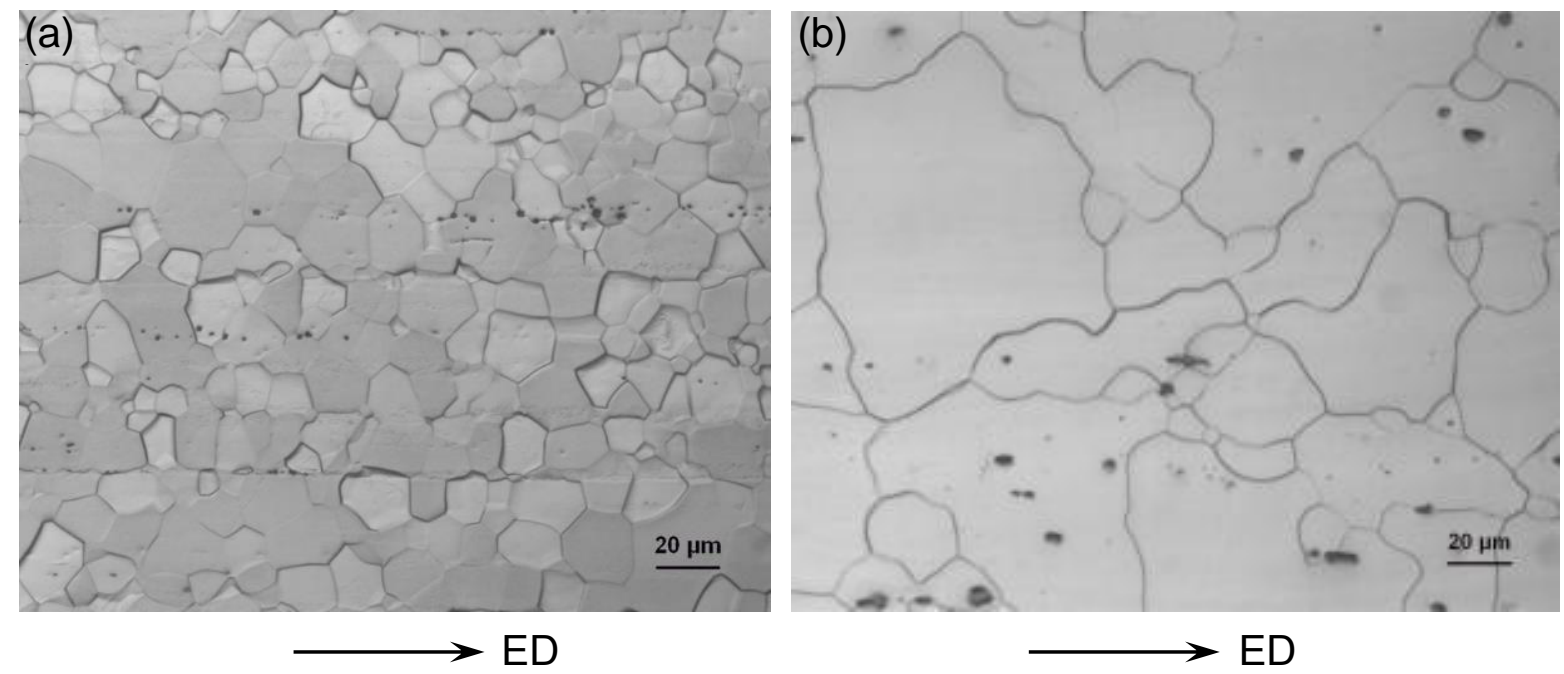

Figure 4.1: Microstructures of extruded (a) GW103K and (b) AM30 alloy.

For example, an addition of $\mathrm{Gd}$ to $\mathrm{AZ3} 1$ alloy resulted in the formation of $\mathrm{Al}_{2} \mathrm{Gd}$ (which was still present after homogenization), and it played an important role in refining the grain size and improving the rolling capability of the alloy [168]; the cerium exhibited a good grain refinement effect on the as-cast AZ31 alloy [170]; and remarkable grain size refinement of $\mathrm{Mg}-1.5 \mathrm{Zn}-0.1 \mathrm{Zr}$ alloy with an addition of erbium was observed through recrystallization during the rolling process [172]. Besides, as seen from Fig. 4.1 no twins were visible in the un-deformed samples. Fig. 4.2(a) shows a typical SEM image of as-extruded sample where it contained three different phases as identified by He et al. [57]: $\alpha-\mathrm{Mg}$ solid solution matrix phase with supersaturated $\mathrm{Gd}+\mathrm{Y}$ elements, $(\mathrm{Gd}+\mathrm{Y})$-rich eutectic compound, and intracrystalline Zr-rich cores. By means of EDS point analysis, the content at points A, B, and C of Fig. 4.2(a) was estimated to be $81.7 \% \mathrm{Mg}-4.9 \% \mathrm{Y}-13.4 \% \mathrm{Gd}, 62.5 \% \mathrm{Mg}-6.1 \% \mathrm{Y}-$ 31.1\% Gd, and 32.4\% Mg-67.6\% Zr, respectively. Similar observations have been reported for as-cast samples of Mg-7Gd-3Y-0.4Zr (GW73K) alloy by Liang et al. [111], cast Mg10Gd-3Y-Zr alloy by Wang et al. [112], as-extruded Mg-11.90Gd-0.81Y-0.44Zr alloy by Zhang et al. [113]. In addition, EDS line scan was performed as shown in Fig. 4.2(b) and Fig. 
4.2(c) which also confirmed the presence of RE-rich particles in the as-extruded alloy, where the middle rectangle-like particle contained both Gd and Y. Such RE-enrichment particles were also observed via TEM which was operated in a microdiffraction mode, as shown in Fig. 4.3(a). Electron microdiffration pattern in Fig. 4.3(b) shows the structure of the particle which was identified as cuboid-shaped (RE-rich) particles (face-centered cubic, fcc with $a=$ $0.56 \mathrm{~nm})$. Similar results were reported in a Mg-10Gd-2Y-0.5Zr alloy [57].
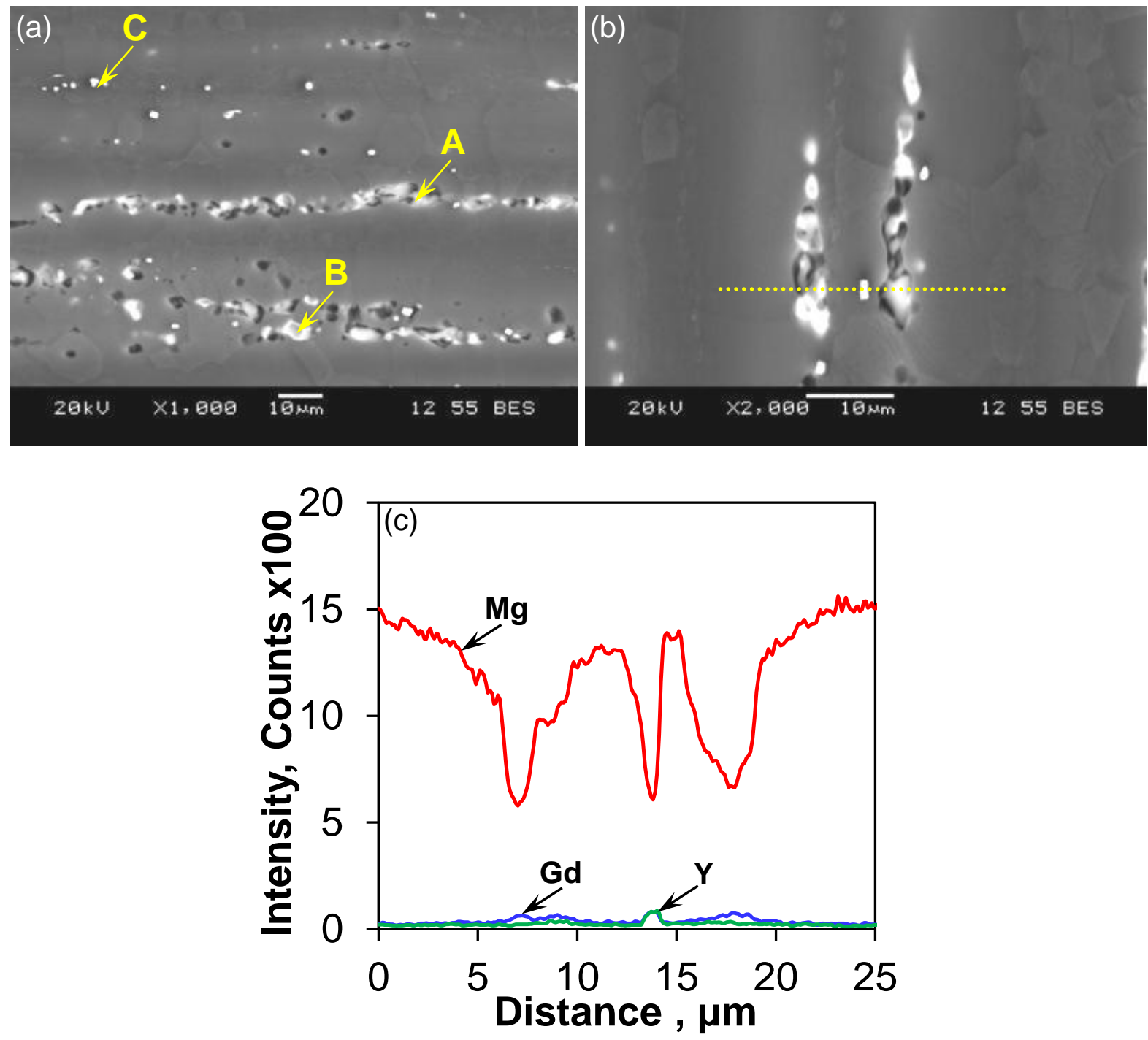

Figure 4.2: (a) SEM backscattered electron image indicating EDS point analysis locations (A, B, and C), (b) SEM backscattered electron image indicating EDS line scan position, and (c) the corresponding EDS line scan results in the GW103K alloy. 

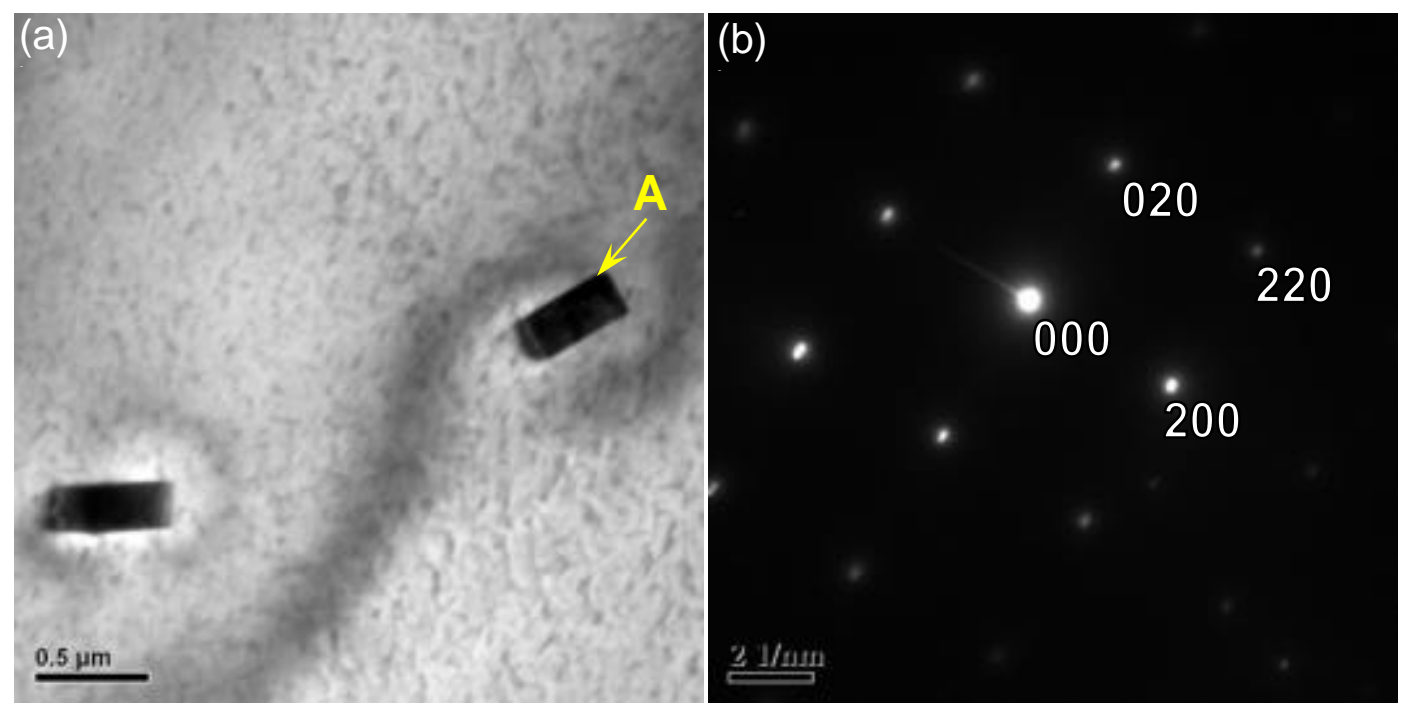

Figure 4.3: Transmission electron micrographs of the as-extruded GW103K alloy: (a) bright-field image taken along [001] zone axis of particle A, and (b) [001] zone axis microdiffraction pattern recorded from particle A.

Fig. 4.4 shows a basal texture where the reflecting surface was parallel to the extrusion direction (ED) and the radial direction (RD). A basal plane fiber texture was observed, where the $c$-axis of most grains was aligned perpendicular to the ED. The peak intensity of the basal plane split to a few degrees around the surface normal direction and some grains with about half of the maximum basal intensity were oriented toward the RD. The presence of such a weaker texture was another benefit of the RE elements added into Mg alloys, as also reported by Stanford and Barnett [60] who observed that microalloying with RE elements can weaken the extrusion texture. A similar weaker texture in Mg-based alloys with the addition of RE elements was reported [114]. In the current GW103K alloy, grain boundary nucleation, coalescence of low-angle boundaries and shear band assisted nucleation are absent (as seen in Fig. 4.1). Zhu et al. [114] reported that only particle-stimulated nucleation was considered to be a factor influencing texture weakening. Indeed, Humphreys [173] observed that the 
high RE content and mixed addition resulted in the precipitation of a large number of the second-phase particles. Such precipitates can hinder the movement of the dislocations so that the stress could concentrate easily near the precipitates, which promoted the particlestimulated nucleation of dynamic recrystalliazation, and weaken the basal texture. In addition, the weakening of the texture was also associated with the appearance of deformation bands containing twins and the restriction of grain growth [120].

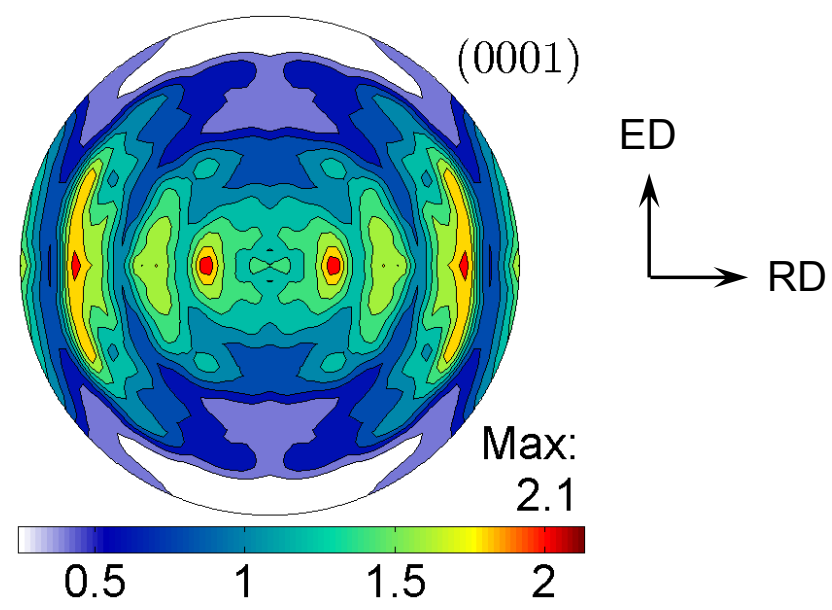

Figure 4.4: (0001) pole figure of as-extruded GW103K alloy, where ED stands for the extrusion direction and $R D$ indicates the radial direction.

\subsection{Uniaxial compression behavior}

The stress-strain curves of the extruded GW103K alloy during compression along the ED are shown in Fig. 4.5(a), where the stress-strain curves of several samples deformed at varying amounts of strain are plotted together to show the reproducibility of the compression tests. An average compressive yield strength of $232 \pm 4 \mathrm{MPa}$ was obtained from the multiple tests, which was higher than the tensile yield strength of $219 \mathrm{MPa}$ for a Mg-10Gd-2Y-0.5Zr alloy [57]. While the tensile percent elongation of $\mathrm{Mg}-10 \mathrm{Gd}-2 \mathrm{Y}-0.5 \mathrm{Zr}$ alloy (extruded at $400^{\circ} \mathrm{C}$ ) 
[57] was higher than the compressive percent shortening of the present as-extruded GW103K alloy, the ultimate compressive strength of the present alloy (493 MPa) was significantly higher than the ultimate tensile strength (305 MPa) of Mg-10Gd-2Y-0.5Zr alloy [57]. This might be attributed to the favorable role of one more percent yttrium added, while all other elements remained the same. Furthermore, in comparison with the compressive test results of an extruded AM30 alloy [36], the extruded GW103K alloy had both higher ultimate compressive strength and ductility (as indicated by the compressive percent shortening). In particular, the as-extruded GW103K alloy exhibited over three-fold higher compressive yield strength than the extruded AM30 alloy, i.e., $232 \mathrm{MPa}$ vs. $71 \mathrm{MPa}$ [36]. Again this reflected the significant beneficial effect of RE elements added into $\mathrm{Mg}$ alloys. Furthermore, unlike the sigmoidal (S-shaped) hardening curves observed in the twinning-dominated deformation, such as in the extruded AM30 Mg alloy [36], the compressive stress-strain curves in Fig. 4.5(a) showed roughly a parabolic-like hardening, indicating a likely change in the deformation mechanisms from the twinning-dominated deformation to the slip-dominated deformation.

To better understand the deformation behavior of the present alloy, the strain hardening rate $d \sigma / d \varepsilon$ (where $\sigma$ is true stress) with respect to true strain $\varepsilon$ from the specimen tested until failure is presented in Fig. 4.5(b) along with the RE-free AM30 extruded Mg alloy [36]. 

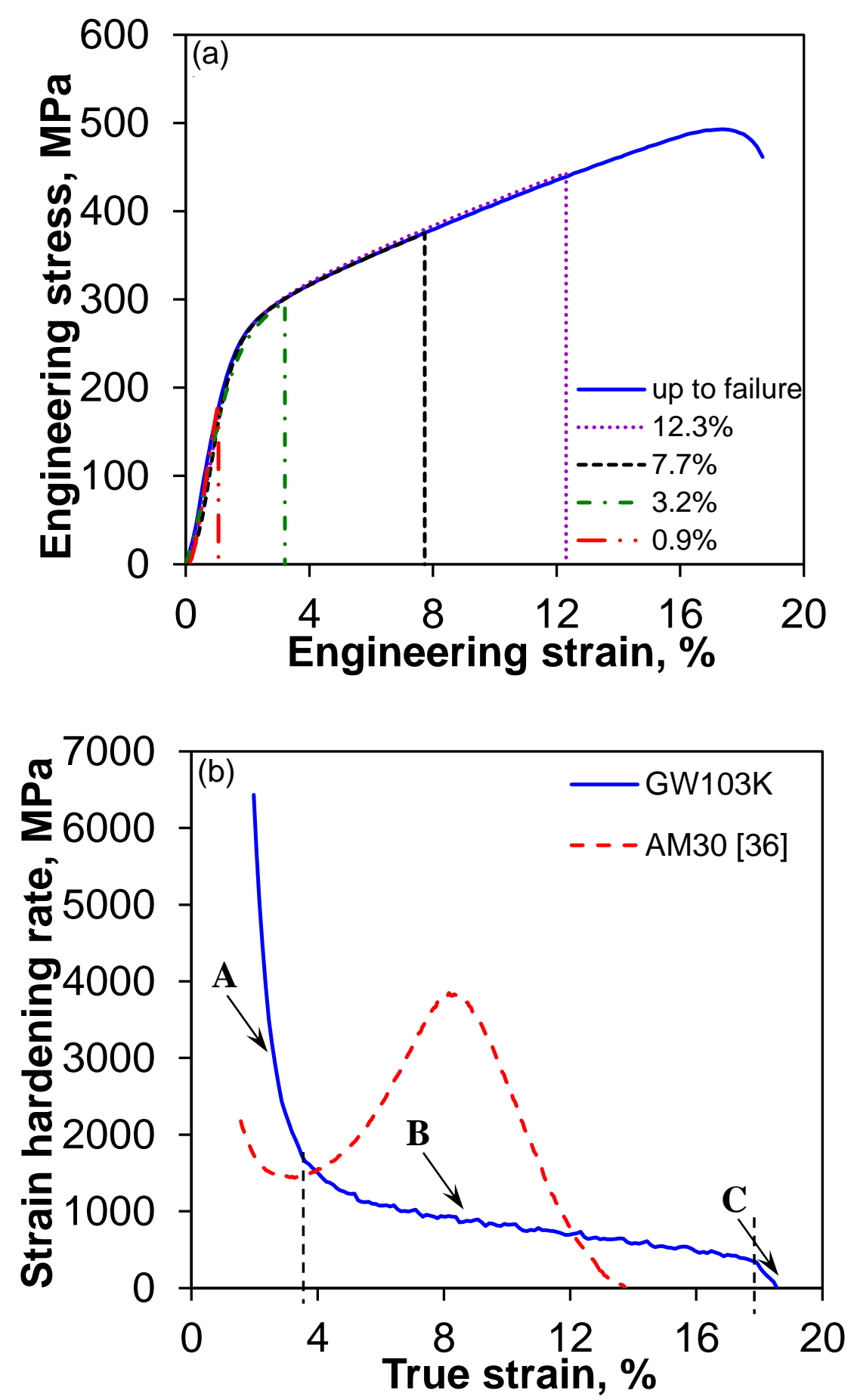

Figure 4.5: (a) Compressive stress-strain curves and (b) strain hardening rate as a function of true strain of as-extruded GW103K alloy in the extrusion direction tested at a strain rate of $10^{-3} \mathrm{~s}^{-1}$. 
Three stages of strain hardening in the extruded GW103K alloy could be distinguished from Fig. 4.5(b). Stage A was characterized by a rapidly decreasing strain hardening rate from the onset of plastic deformation up to a strain of $\sim 4 \%$, followed by stage B with a fairly flat and almost linear strain hardening rate over a large range of strain up to $\sim 18 \%$, and then stage $\mathrm{C}$ with an decreasing strain hardening rate again until failure. Three stages of strain hardening rate have been reported by Sarker and Chen [36] where stage A and stage C had a falling trend, as observed in the present study as well. However, as seen from Fig. 4.5(b), the value of strain hardening rate in stage A was much higher in the present extruded GW103K alloy than in the extruded AM30 alloy; stage B of the AM30 Mg alloy was totally different from that of the present alloy. Similar trend in the strain hardening rate to the GW103K alloy has also been observed in extruded Mg-Y-Nd alloy [174] and ZK60 Mg alloy [175], where the strain hardening rate started with a similarly steep decrease at small strains and then approached a linear/steady state with a very low value over a large range of strain. The significant difference in the strain-hardening rate in stages A and B between the present extruded GW103K and AM30 alloys could be understood on the basis of the amount of twins formed during compression and the subsequent interactions between twin and twin, twin and dislocation, twin-precipitate, etc., which will be discussed in the following section.

\subsection{Strain hardening behavior}

To understand the strain hardening behavior, the deformation characteristics of the samples compressed to different amounts of strain were examined. Fig. 4.6 shows the change of twin volume fraction with the applied compressive strain, together with some typical images 
showing the visual change of twins. It is seen from image (a) in Fig. 4.6 that at a compressive strain of $\varepsilon=0.9 \%$, no twins were visible, whereas at $\varepsilon=3.2 \%$ a very small number of twins were observed in some grains (image (b) in Fig. 4.6). The twins formed during compression in the extruded Mg alloys along the ED direction has been identified as $\{10 \overline{1} 2\}$ extension twins in a number of publications [36,114]. At a strain of $\varepsilon=7.7 \%$, more twins were observed in more grains (image (c) in Fig. 4.6). When the specimen was deformed at a compressive strain of $\varepsilon=12.3 \%$, the twin volume fraction reached its maximum of $\sim 0.04$ (image (d) in Fig. 4.6). In addition, Fig. 4.7 shows the typical SEM micrographs at strain levels of $3.2 \%$, and $12.3 \%$, respectively. It is obvious that the compressive deformation at a strain of $12.3 \%$ resulted in more twins than that at a strain of 3.2\%.

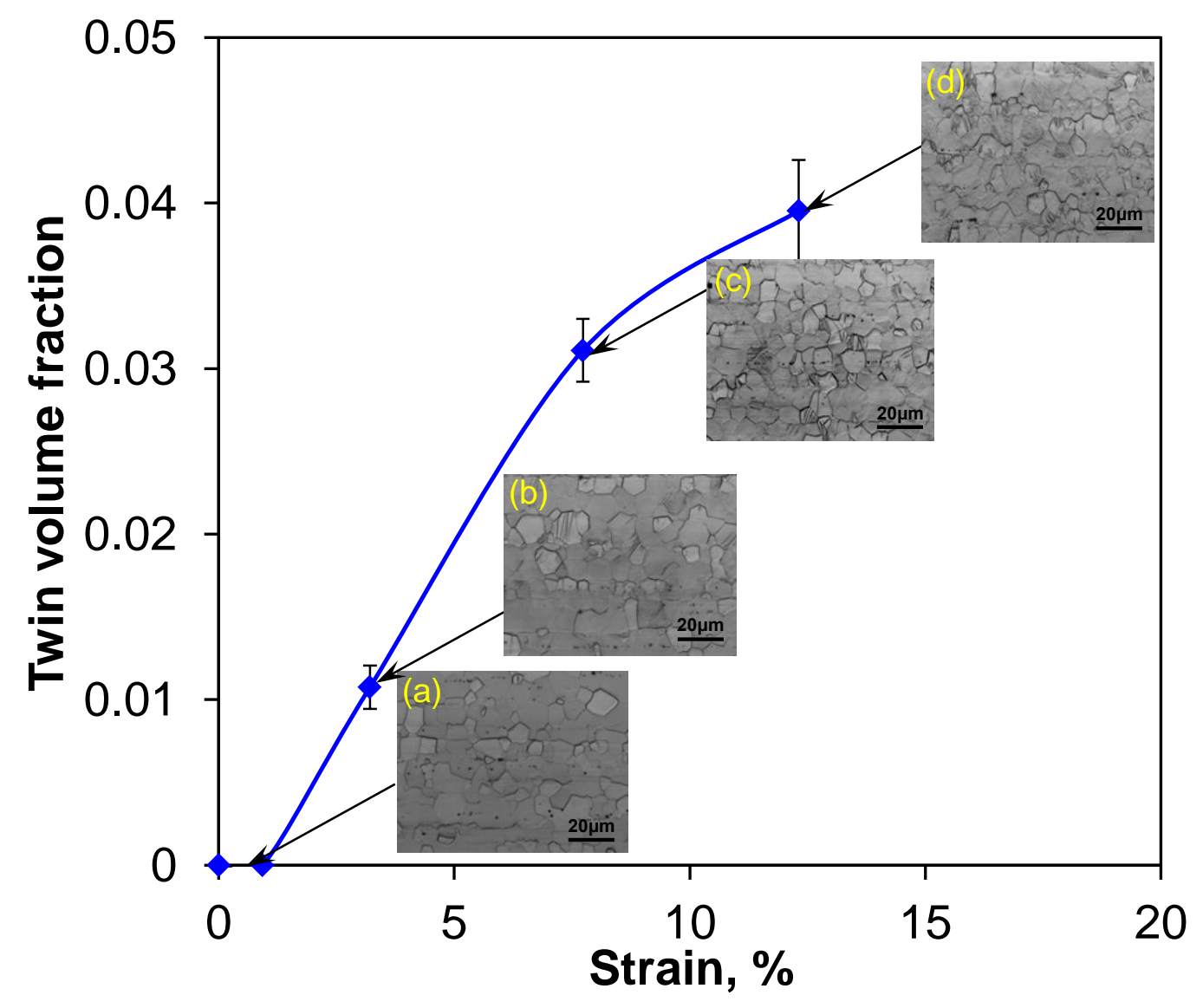


Figure 4.6: Variation of the volume fraction of twins with the applied strain during compression in the as-extruded GW103K alloy, where some typical micrographs at different strain levels are inserted to show the evolution of deformation twins.
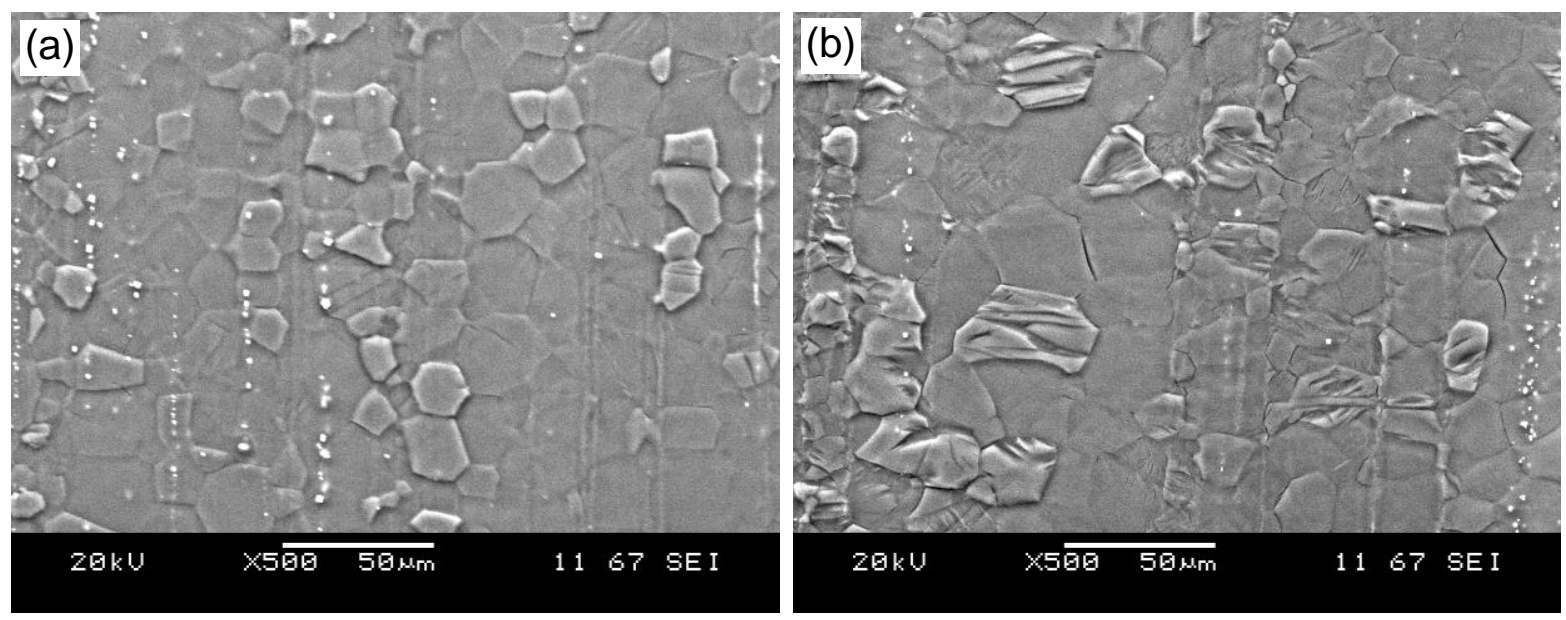

Figure 4.7: Typical SEM micrographs of as-extruded GWIO3K alloy deformed in compression at a strain level of (a) $3.2 \%$, and (b) $12.3 \%$.

\subsection{Effects of RE elements on strain hardening}

Plastic deformation of $\mathrm{Mg}$ alloy is mainly determined by the HCP structure and strongly affected by the crystallographic texture. Thus, crystallographic texture would be one of the key factors influencing the deformation behavior of GW103K alloy. As the orientation of each grain changes, the combination of active slip system changes [176,177]. When a change of active slip systems occurs, the flow stress will rise which change the hardening [177]. Most grains re-orient to a stable orientation so that the effect of flow stress would be expected to saturate and not lead to any new strain hardening at very large strains. As the addition of RE elements offers an avenue to control or change the crystallographic texture, it 
would thus affect the slip and twinning systems and change the deformation behavior. It was observed that, in the case of a weak basal texture (Fig. 4.4), it is expected that 〈a〉 dislocation slip with a relatively low critical resolved shear stresses (CRSS) would contribute more to accommodate the deformation and twin formation will be more difficult [123]. Table 4.1 lists a summary of CRSS values reported for Mg and its alloys. Clearly, twinning has much lower CRSS than basal slip. For example, as compared to the CRSS of basal slip (45 $\mathrm{MPa}$ ), twinning has three times lower CRSS (15 MPa), and prismatic slip has two to three times higher CRSS [178]. Moreover, Hantzsche et al. [120] reported that the deformation texture changes at a higher $\mathrm{Y}$ content, which is related to an increasing activity of $\langle c+a\rangle$ slip and thus can also reflect in the twinning activity. There is a general agreement that a smaller grain size inhibited twinning [179] or grain size had a significant effect on the tendency of twinning since the energy required to form twin interfaces is particularly high in Mg [180]. Finer grains, which have a higher surface-to-volume ratio, increase the difficulty of twin nucleation and thereby suppress twinning by limiting the sizes of the twins [181]. The addition of RE elements and $\mathrm{Zr}$ in the present GW103K alloy led to a fairly fine grain size, as seen from Fig. 4.1. Thus in the present as-extruded GW103K alloy, the grain size would be another important factor influencing the incidence of deformation twinning. It was also reported that it is common for the twinning stress to increase with decreasing grain size more rapidly than the stress required to activate slip [182]. As a result, the formation of deformation twins was more difficult in the GW103K alloy as compared with AM30 alloy [36] due to the grain refinement role of RE elements as mentioned in the above section 4.1. In addition to the effect of texture and grain size, the presence of precipitates has also been 
reported to impede the process of twinning in $\mathrm{Mg}$ alloys [183]. The details of this mechanism will be discussed in following chapter (Chapter 6).

Table 4.1: Critical resolved shear stresses (CRSS) reported for Mg and its alloys.

\begin{tabular}{|c|c|c|c|c|}
\hline Samples & Condition/Experimental & Invariant plane & $\begin{array}{l}\text { CRSS } \\
(\mathrm{MPa})\end{array}$ & Ref. \\
\hline $\mathrm{Mg}$ & single crystal/tensile & $\begin{array}{c}\text { \{10-12\} } \\
\text { prismatic }\end{array}$ & $\begin{array}{c}2 \\
39.2\end{array}$ & 184 \\
\hline $\mathrm{Mg}-\mathrm{Li}$ & single crystal & $\{10-11\}$ & 114 & 185 \\
\hline $\mathrm{Mg}$ & $\begin{array}{l}\text { single crystal } \\
\text { /compression }\end{array}$ & $\{10-11\}$ & $76-153$ & 186 \\
\hline $\mathrm{Mg}-3 \mathrm{Al}-1 \mathrm{Zn}$ & compression & $\begin{array}{c}\text { "basal } \\
:\{10-12\} \\
:<c+a> \\
\text { :prismatic }\end{array}$ & $\begin{array}{c}{ }^{*} 1 \\
: 0.3 \\
: 2.9 \\
: 2.4\end{array}$ & 187 \\
\hline $\mathrm{Mg}$ & single crystal & basal & 0.81 & 188 \\
\hline AZ31B & polycrystal & $\begin{array}{c}\text { basal } \\
\{10-12\} \\
\text { prismatic }\end{array}$ & $\begin{array}{c}45 \\
15 \\
110\end{array}$ & 178 \\
\hline $\begin{array}{c}\mathrm{Mg} 7.7 \text { at. pct } \\
\mathrm{Al}\end{array}$ & polycrystal & $\{10-12\}$ & $65-75$ & 189 \\
\hline
\end{tabular}

"Note: CRSS ratio.

It was reported that at large compressive strains the spacing between the second-phase decreased [176]. If the second-phase is hard enough to block the dislocation flow or twinning in the matrix, the mean free path for dislocation motion and twinning decreases strongly with strain and leads to rises of the flow stress by the Hall-Petch effect, thus contributing to the strain hardening $[176,179]$. In addition, strain hardening is by nature associated with elastic and inelastic incompatibilities between matrix and particles during plastic deformation. The 
direction of this resistance is opposite to the applied stress, and therefore opposes the bulk slip and twinning, contributing to the overall strain hardening as well. Furthermore, the plastic relaxation of the matrix at the RE particle interface could create both deformation twins and a high local dislocation density, which are obstacles to the moving dislocations, and would also affect the strain hardening [190]. Similar result regarding the effect of second-phase particles on the strain hardening has also been reported in ref. [120]. Therefore, the presence of RE-containing particles in conjunction with finer grain sizes and weaker texture is responsible for the much higher strain hardening rate in stage A in the GW103K alloy (Fig. 4.5(b)), due to the increased difficulty on the slip of dislocations and the formation of twins.

The concept of dislocation hardening could also be used to explain the linear portion in stage B of the strain hardening rate curve of GW103K alloy, as shown in Fig. 4.5(b). Several models have been proposed to explain stage B (in some studies which is referred to as stage IV) [177,191-193]. It was reported that stage B might be associated with microscopic instabilities such as microbands commonly observed at large strains in copper [191]. The cell model proposed by Nix et al. [193] indicated that plastic straining at low temperatures leads to the accumulation of dislocation debris such as dipoles and loops. The accumulation of dipolar debris may occur as part of the glide process and it may also occur as part of the dislocation rearrangements due to dynamic recovery [192]. However, the rate of dislocation rearrangement inside the cell walls is expected to be affected by the debris left and would have forward internal stresses within the cell walls that lead to rearrangement or even annihilation of dislocation segments in this region. If the effect of the accumulating is to raise 
the flow stress within the cell walls of the dislocation substructure, then superposition of flow stress would contributed in hardening and ultimately leads to a finite hardening rate in stage B (fairly flat linear or sometimes constant). Similar observations of stage IV or stage B in different extruded $\mathrm{Mg}$ alloys were reported in several studies [174,175]. The above discussion indicated that the addition of RE elements was able to affect significantly the deformation behavior of the extruded Mg alloys by predominantly suppressing the twinning due to the finer grain size, the relatively more random crystallographic texture, and the presence of RE-containing precipitates. However, more studies related to the dislocation-twin interactions, twin-particle interactions, twin-grain boundary interactions from both experimental and modeling/theoretical aspects are needed.

\subsection{Summary}

The following conclusions can be drawn from this investigation:

1. A rapidly decreasing strain hardening rate up to a strain level of $\sim 4 \%$ (stage A), followed by a fairly flat linear strain hardening rate over an extended strain range from $\sim 4 \%$ to $~ 18 \%$ (stage B) was observed.

2. Stage $\mathrm{C}$ was represented by a decreasing strain hardening rate just prior to failure. The amount of twins observed in the as-extruded GW103K alloy was much less extensive than that in the RE-free extruded AM30 Mg alloy.

3. The weaker crystallographic texture, refined grain size, and RE-containing second-phase particles were likely responsible for the much higher strain hardening rate in stage $\mathrm{A}$ in the 
as-extruded GW103K alloy due to the increased difficulty on the formation of twins and the slip of dislocations at lower strains.

4. The occurrence of fairly flat linear strain hardening rate in stage B at higher strains was likely associated with the presence of dislocation debris and twin debris (or residual twins) resulting mainly from dislocation-twin interactions as well as the interactions between the dislocations/twins and RE-rich particles and grain boundaries. 


\section{CHAPTER 5}

\section{A MODIFIED JOHNSON-COOK CONSTITUTIVE RELATIONSHIP FOR A RARE-EARTH CONTAINING MAGNESIUM ALLOY}

To understand the deformation behavior of Mg alloys in a varying range of strain rates and temperatures, it is necessary to develop constitutive relationships related to the stress, strain, temperature and strain rate $[194,195]$, which are also important in designing the load-bearing structural components [196]. Ideally, such equations should involve a reasonable number of material constants that can be evaluated using limited experimental data and should be able to represent the flow behavior of the material with sufficient accuracy [197]. Several empirical, semi-empirical, and physically based flow stress models have been proposed to predict the constitutive behavior of metals and alloys [198-202]. For example, Johnson-Cook equation $[199,201]$ was successfully used over a wide range of strain rates and forming temperatures for body-centered cubic (bcc) and face-centered cubic (fcc) metals due to its simple multiplication form [203,204]. Another model (Zerili-Armstrong model [200]) which is a modified version of Johnson-Cook model was also used. It has also been reported that the Johnson-Cook equation could be used to study the plastic response of not only bcc or fcc metals but also hcp metals [196]. Hou and Wang [196] performed the dynamic impact tests of an extruded $\mathrm{Mg}-10 \mathrm{Gd}-2 \mathrm{Y}-0.5 \mathrm{Zr}$ alloy using a split Hopkinson pressure bar apparatus at high strain rates in-between $8 \times 10^{2} \mathrm{~s}^{-1}$ and $4 \times 10^{3} \mathrm{~s}^{-1}$ at varying temperatures in comparison with a quasi-static compression test, and proposed an empirically based model by changing the temperature term in the original Johnson-Cook model based on their experimental results. Liu et al. [205] also studied the high strain rate compression behavior and proposed a 
constitutive relation using the modified Johnson-Cook model for the dynamic deformation behavior of an extruded Mg-12Gd-3Y-0.5Zr alloy. In spite of these reports on the high strain rate deformation behavior, the studies on the constitutive relationships of wrought $\mathrm{Mg}$ alloys are still limited [194,206,207], and especially it is unknown what constitutive equation would be suitable for describing the flow behavior of RE-Mg alloys based on the more commonly used low strain rate deformation tests. This part of the dissertation was aimed to study the deformation behavior of an extruded GW103K in compression at relatively low strain rates, and explore a constitutive relation to quantify its deformation behavior from a different angle, i.e., based on the Johnson-Cook equation and a recently adopted strain hardening method.

\subsection{Compression test}

A typical engineering stress-strain $(\sigma-\varepsilon)$ curve of the as-extruded GW103K alloy in compression along the ED is shown in Fig. 5.1, where the curve of a RE-free extruded AM30 $\mathrm{Mg}$ alloy [108] is plotted as well. It is seen that the present RE-containing alloy had an appreciably higher compressive yield strength, indicating a significant favorable role of RE elements added into $\mathrm{Mg}$ alloys. Also, the $\sigma-\varepsilon$ curve of the present alloy appeared to be normal, unlike the skewed shape of AM30 curve that was attributed to the occurrence of extensive twinning in the initial stage followed by the twin growth phenomena stemming from the strong twin-dislocation interactions, leading to an texture transformation in the REfree extruded magnesium alloy in compression [108]. The attempt of this part of the dissertation was thus limited to establish a constitutive relationship for the RE-containing $\mathrm{Mg}$ 
alloys in compression, leaving an apparently more complicated constitutive relationship of RE-free extruded Mg alloys for further studies.

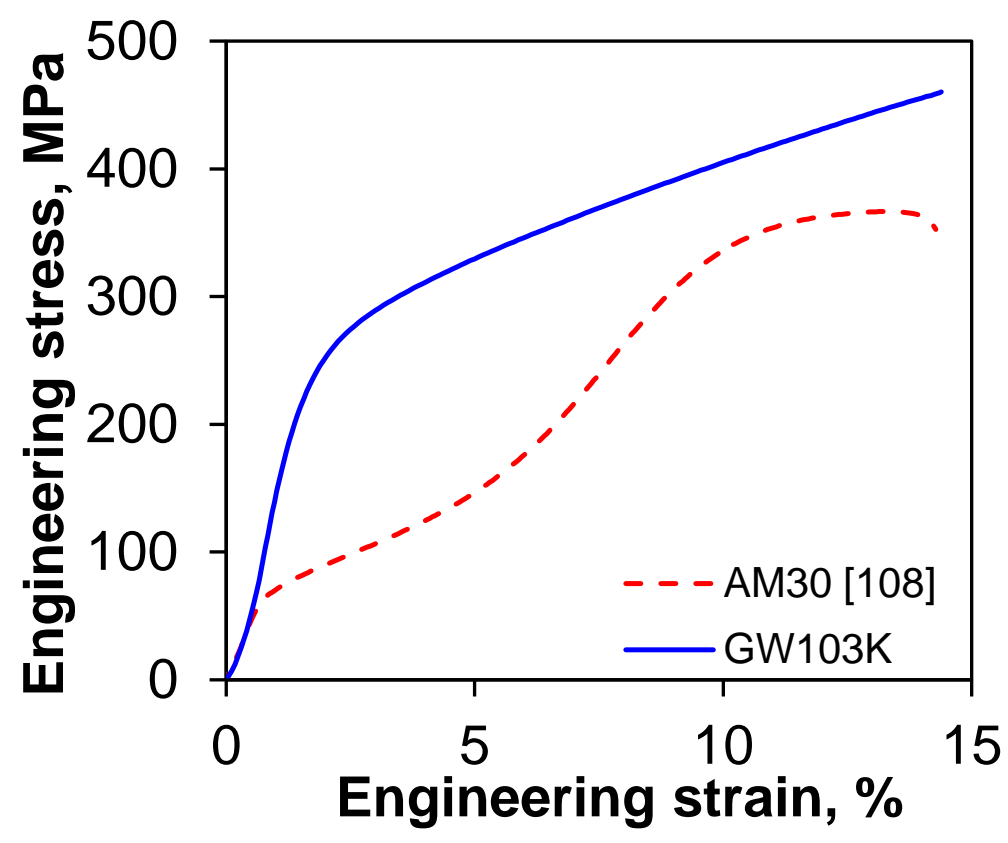

Figure 5.1: Compressive stress-strain curves of the extruded GW103K alloy and RE-free AM30 alloy [108].

\subsection{Constitutive model}

Johnson and Cook [199,201] proposed an empirically based constitutive model for materials subjected to large strains, high strain rates and high temperatures which has become one of the most widely-used strain-rate dependent flow stress models. This model is comparatively simpler than other models since the effects of strain, strain rate, and temperature on the plastic deformation of the materials are simply incorporated in a multiplication form $[196,199,201]$. According to the original Johnson-Cook model the flow stress could be expressed as follows [199,201], 


$$
\sigma=f(\varepsilon) f(\dot{\varepsilon}) f(T)=\left(A+B \varepsilon^{n}\right)\left(1+C \ln \frac{\dot{\varepsilon}}{\dot{\varepsilon}_{o}}\right)\left[1-\left(T^{*}\right)^{m}\right]
$$

where $\sigma$ is the flow stress of material, $\dot{\varepsilon}$ is the applied strain rate, $\dot{\varepsilon}_{o}$ is a reference strain rate, $A, B, C, n$ and $m$ are material constants. $T^{*}$ can be expressed as follows,

$$
T^{*}=\frac{T-T_{r}}{T_{m}-T_{r}}
$$

where $T$ is the current absolute temperature, $T_{r}$ is room temperature, and $T_{m}$ is melting temperature. It is clear that this model combines the effects of strain rate, strain hardening, and thermal softening in a single equation. While the Johnson-Cook model was mainly used to describe the deformation behavior of materials at high strain rates and high temperatures $[196,208]$, it could be extended to describe the flow behavior of materials in the quasi-static tensile tests at relatively low strain rates [209]. It is thus inferred that the flow behavior of common materials could also be described by a modified Johnson-Cook model. In the present study the Johnson-Cook model was used as a starting point to construct the constitutive relationship for the RE-containing $\mathrm{Mg}$ alloy. Since the present tests were performed at room temperature, equ.(5.1) could be simplified as,

$$
\sigma=\left(A+B \varepsilon^{n}\right)\left(1+C \ln \frac{\dot{\varepsilon}}{\dot{\varepsilon}_{o}}\right)
$$

To quantify better the stress response, the method proposed by Afrin et al. [99] is used in the present formulation. In their method [99] the yield point was excluded in the determination of strain hardening exponent and coefficient, i.e., the terms of $\left(\sigma-\sigma_{\mathrm{y}}\right)$ and $\left(\varepsilon-\varepsilon_{\mathrm{y}}\right)$ were used to replace the true stress $\sigma$ and true strain $\varepsilon$ in the Hollomon relationship, where $\sigma_{\mathrm{y}}$ is the yield stress and $\varepsilon_{\mathrm{y}}$ is the yield strain. Equ.(5.3) then becomes, 


$$
\sigma=\left[A+B\left(\varepsilon-\varepsilon_{y}\right)^{n *}\right]\left(1+C \ln \frac{\dot{\varepsilon}}{\dot{\varepsilon}_{o}}\right)
$$

where $n^{*}$ is a modified strain hardening exponent. This becomes a generalized constitutive equation used to describe the strain rate dependent flow stress at room temperature, but it requires the determination of several material constants. The steps of how to determine the constants for a particular material are specified below. The first step is to select a reference strain rate $\dot{\varepsilon}_{o}$ and calculate the flow stress at this specific strain rate. Then equ.(5.4) becomes,

$$
\sigma=\sigma_{o}\left(\dot{\varepsilon}_{o}\right)+B\left(\varepsilon-\varepsilon_{y}\right)^{n^{*}}
$$

where $\sigma_{o}\left(\dot{\varepsilon}_{o}\right)(=A)$ is the yield stress of the material at the reference strain rate $\dot{\varepsilon}_{o}$. Rearranging and taking log of equ.(5.4a) yields,

$$
\log \left[\sigma-\sigma_{o}\left(\dot{\varepsilon}_{o}\right)\right]=\log B+n^{*} \log \left(\varepsilon-\varepsilon_{y}\right)
$$

$B$ and $n^{*}$ can thus be obtained via plotting and fitting $\log \left[\sigma-\sigma_{o}\left(\dot{\varepsilon}_{o}\right)\right]$ vs. $\log \left(\varepsilon-\varepsilon_{y}\right)$ over the range from the yield stress to the peak true stress at the reference strain rate. The second step is to evaluate parameter $C$ in equ.(5.4) when the strain is the yield strain $\varepsilon_{y}$. In this case equ.(5.4) can be simplified as,

$$
\frac{\sigma(\dot{\varepsilon})}{\sigma_{o}\left(\dot{\varepsilon}_{o}\right)}-1=C \ln \frac{\dot{\varepsilon}}{\dot{\varepsilon}_{o}}
$$

Using the yield stress data at different strain rates, $C$ can be obtained from the slope of $\left[\frac{\sigma(\dot{\varepsilon})}{\sigma_{o}\left(\dot{\varepsilon}_{o}\right)}-1\right]$ vs. $\ln \frac{\dot{\varepsilon}}{\dot{\varepsilon}_{o}}$ plot. Substituting all the parameters $\left(A=\sigma_{o}\left(\dot{\varepsilon}_{o}\right), B, n^{*}\right.$, and $\left.C\right)$ into equ.(5.4) one can obtain the constitutive relationship for a specific material. 


\subsection{Model validation}

In the present study a reference strain rate of $1 \times 10^{-3} \mathrm{~s}^{-1}$ is selected, which is in reference to the ASTM E8/E8-11 standard [164] where a strain rate of $2.5 \times 10^{-4} \mathrm{~s}^{-1}$ for determining the yield strength and $0.83 \sim 8.33 \times 10^{-3} \mathrm{~s}^{-1}$ for the tensile strength is specified. Using the true stress-strain curve of the present RE-containing Mg alloy measured at this reference strain rate following equ.(5.4(b)), $B$ and $n^{*}$ can be determined to be 537 and 0.56 , respectively. The value of $C$ can be obtained to be 0.0063 by a linear fitting following equ.(5.4c), using the yield stresses at different strain rates (from $1 \times 10^{-1} \mathrm{~s}^{-1}$ to $1 \times 10^{-4} \mathrm{~s}^{-1}$ ). Inserting all the values of the parameters into equ.(5.4) yields a constitutive relationship for the present GW103K alloy as follows,

$$
\sigma=\left[226+537\left(\varepsilon-\varepsilon_{y}\right)^{0.56}\right]\left(1+0.0063 \ln \frac{\dot{\varepsilon}}{\dot{\varepsilon}_{o}}\right)
$$

The true stress-true strain curves calculated from equ.(5.5) are plotted in Fig. 5.2(a-d), in comparison with the experimentally-determined true stress-true strain curves at different strain rates and with an expression given in ref. [205] for a Mg-12Gd-3Y-0.5Zr (GW123K) Mg alloy. Several commonly-used flow stress equations:

$$
\begin{gathered}
\sigma=K \varepsilon^{n}, \text { Hollomon [210], } \\
\sigma=\sigma_{y}+K_{1} \varepsilon^{n_{1}}, \text { Ludwik [211], } \\
\sigma=\sigma_{y}+K^{*}\left(\varepsilon-\varepsilon_{y}\right)^{n^{*}}, \text { Afrin et al. }[99]
\end{gathered}
$$

have also been plotted in Fig. 5.2. The parameters obtained for the present RE-containing Mg alloy tested at different strain rates based on equ.5.6(a-c) are listed in Table 5.1. 

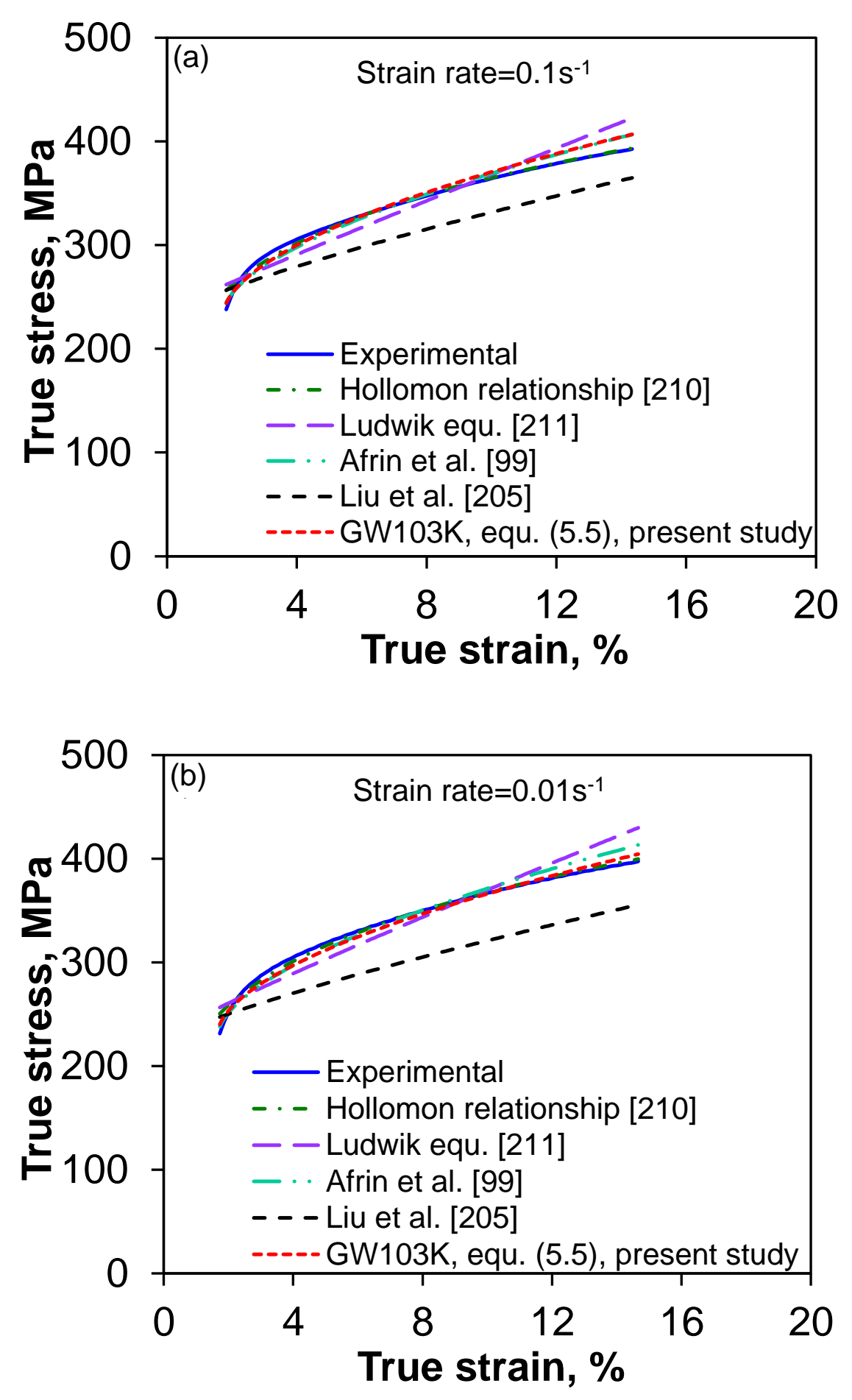

Figure 5.2: Comparison of the calculated true stress-strain curves of the extruded GW103K alloy with those experimentally determined at different strain rates of (a) $0.1 \mathrm{~s}^{-1}$, (b) $0.01 \mathrm{~s}^{-1}$, (c) $0.001 \mathrm{~s}^{-1}$, and (d) $0.0001 \mathrm{~s}^{-1}$ (continued.). 

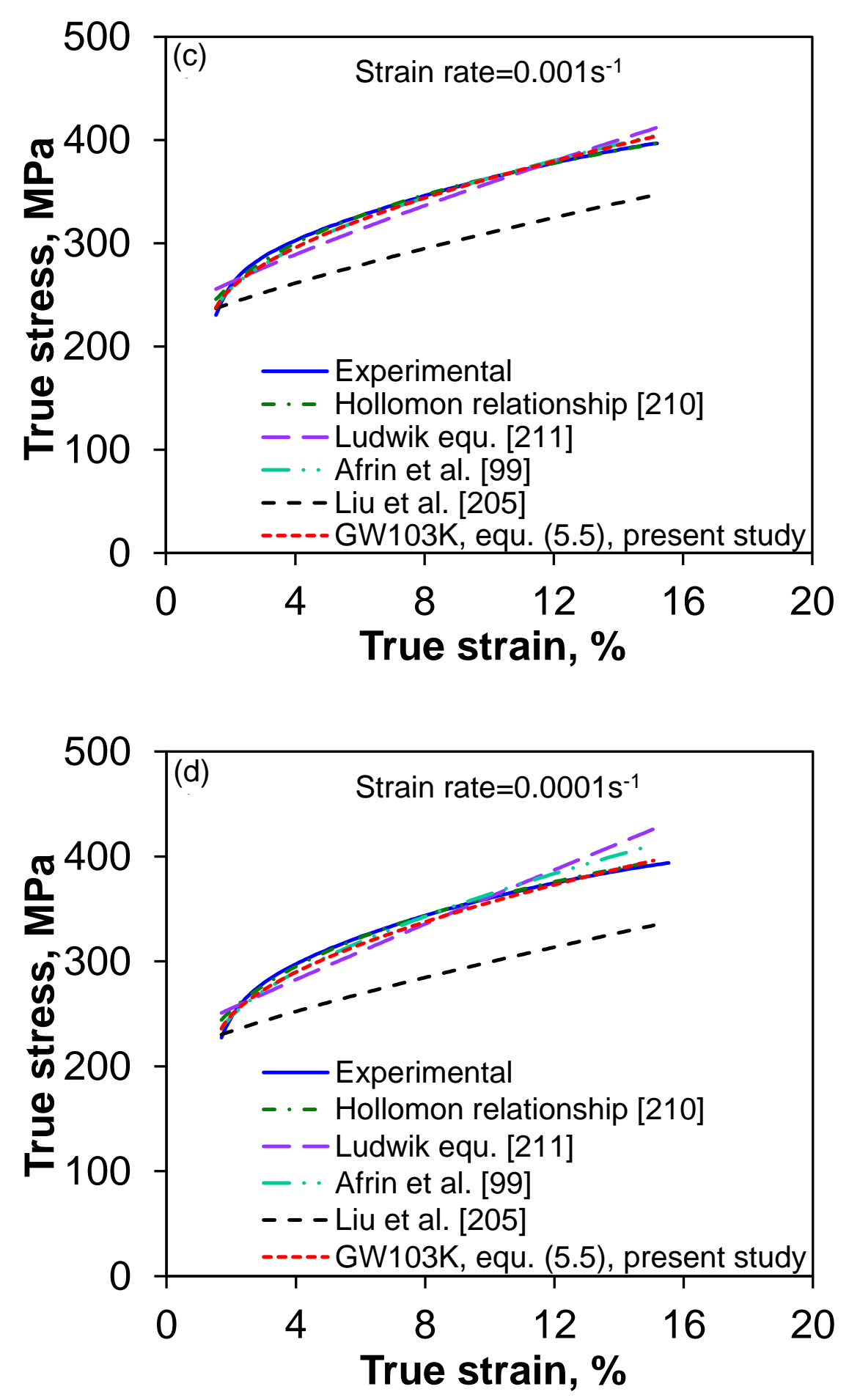

Figure 5.2: Comparison of the calculated true stress-strain curves of the extruded GW103K alloy with those experimentally determined at different strain rates of (a) $0.1 \mathrm{~s}^{-1}$, (b) $0.01 \mathrm{~s}^{-1}$, (c) $0.001 \mathrm{~s}^{-1}$, and (d) $0.0001 \mathrm{~s}^{-1}$. 
To examine the accuracy of the modified Johnson-Cook equation, the relative errors between the predicted flow stress $\left(\sigma_{p}\right)$ and measured flow stress $\left(\sigma_{m}\right)$,

$$
\text { error }=\frac{\sigma_{p}-\sigma_{m}}{\sigma_{m}} \times 100 \%,
$$

were calculated at different values of true strains and strain rates, as shown in Table 5.2. It is seen from Fig. 5.2 and Table 5.2 that the present modified Johnson-Cook constitutive equation is in good agreement with the experimental data that appears better than other common flow stress equations and could provide a fairly good estimation of the flow stresses for the present RE-containing magnesium alloy with a standard deviation of $1.83 \%$. Moreover, the mean difference between the calculated and experimental flow stress are $1.82 \%,-0.07 \%$, and $1.66 \%$ for true strain of $5 \%, 10 \%$, and $14 \%$, respectively. It is seen that the modified Johnson-Cook constitutive equation

Table 5.1: The material constants in the equations of Hollomon [210], Ludwik [211], and Afrin et al. [99] for the flow stress curves at different strain rates.

\begin{tabular}{ccccccc}
\hline & \multicolumn{2}{c}{ Hollomon [210], } & \multicolumn{2}{c}{ Ludwik [211], } & \multicolumn{2}{c}{ Afrin et al. [99], } \\
Strain rate, $\mathrm{s}^{-1}$ & \multicolumn{2}{c}{$\sigma=K \varepsilon^{n}$} & \multicolumn{2}{c}{$\sigma=\sigma_{y}+K_{1} \varepsilon^{n_{1}}$} & \multicolumn{2}{c}{$\sigma=\sigma_{y}+K^{*}\left(\varepsilon-\varepsilon_{y}\right)^{n^{*}}$} \\
& $K, \mathrm{MPa}$ & $n$ & $K_{1}, \mathrm{MPa}$ & $n_{1}$ & $K^{*}, \mathrm{MPa}$ & $n^{*}$ \\
\hline $1 \times 10^{-1}$ & 589 & 0.21 & 1202 & 0.96 & 579 & 0.59 \\
$1 \times 10^{-2}$ & 608 & 0.22 & 1187 & 0.93 & 605 & 0.58 \\
$1 \times 10^{-3}$ & 589 & 0.21 & 866 & 0.82 & 543 & 0.56 \\
$1 \times 10^{-4}$ & 599 & 0.22 & 1206 & 0.95 & 640 & 0.62 \\
\hline
\end{tabular}


Table 5.2: A comparison between the predicted $\left(\sigma_{p}\right)$ and measured $\left(\sigma_{m}\right)$ flow stress values.

\begin{tabular}{|c|c|c|c|c|c|}
\hline \multirow{3}{*}{$\begin{array}{c}\text { Absolute value } \\
\text { of } \\
\text { true strain, \% }\end{array}$} & Strain rate, & Measured flow & Predicted flow & Relative & Mean \\
\hline & $\mathrm{s}^{-1}$ & stress $\left(\sigma_{m}\right)$ & stress $\left(\sigma_{p}\right)$, & error, $\%$ & error $(\%)$ \\
\hline & & $\mathrm{MPa}$ & $\mathrm{MPa}$ & & \\
\hline \multirow{4}{*}{5} & $1 \times 10^{-1}$ & 318 & 316 & -0.63 & \\
\hline & $1 \times 10^{-2}$ & 319 & 312 & -2.19 & -1.82 \\
\hline & $1 \times 10^{-3}$ & 316 & 310 & -1.90 & \\
\hline & $1 \times 10^{-4}$ & 313 & 305 & -2.56 & \\
\hline \multirow{4}{*}{10} & $1 \times 10^{-1}$ & 365 & 371 & 1.64 & \\
\hline & $1 \times 10^{-2}$ & 367 & 366 & -0.27 & -0.07 \\
\hline & $1 \times 10^{-3}$ & 363 & 362 & -0.28 & \\
\hline & $1 \times 10^{-4}$ & 362 & 357 & -1.38 & \\
\hline \multirow{4}{*}{14} & $1 \times 10^{-1}$ & 391 & 405 & 3.58 & \\
\hline & $1 \times 10^{-2}$ & 393 & 399 & 1.53 & 1.66 \\
\hline & $1 \times 10^{-3}$ & 390 & 395 & 1.28 & \\
\hline & $1 \times 10^{-4}$ & 387 & 388 & 0.26 & \\
\hline
\end{tabular}




\section{$5.4 \quad$ Summary}

The following conclusions can be drawn from this investigation:

1. The deformation behavior of a RE-containing $\mathrm{Mg}$ alloy in compression at different strain rates was evaluated, and a modified Johnson-Cook constitutive equation was proposed to predict the flow stresses.

2. Comparisons between the predicted and experimental results, along with some commonly-used flow stress equations, confirmed that the modified Johnson-Cook constitutive equation was able to estimate the flow stresses for the RE-containing $\mathrm{Mg}$ alloy fairly accurately with a standard deviation of about $1.83 \%$. 


\title{
CHAPTER 6
}

\section{CYCLIC DEFORMATION BEHAVIOR OF EXTRUDED GW103K}

\begin{abstract}
ALLOY
As mentioned earlier in Chapter 1 and 2, the unfavorable tension-compression yield asymmetry could be altered due to the RE elements additions, however, the potential advantage of such extruded RE-Mg alloys as structural components under dynamic cyclic loading condition has not yet been well explored. Studies on the strain-controlled low cycle fatigue behavior of RE-Mg alloys remain limited to date [58,147-149]. It is unclear how and to what extent the RE-elements can change the tension-compression yield asymmetry which generally occurs in the RE-free extruded $\mathrm{Mg}$ alloys, whether RE-Mg alloys exhibit cyclic hardening or softening, and via what mechanisms the RE-elements affect the cyclic deformation behavior and fatigue life. In addition, to the author's knowledge, no systematic studies have been conducted to understand the effect of heat treatment conditions on the low cyclic fatigue behavior of RE-containing Mg alloys in the open literature. Also, it has not been fully understood how the cyclic deformation and low cycle fatigue resistance change due to heat treatment, whether the heat-treated RE-Mg alloy exhibits cyclic hardening or softening, and what is the effect of the heat treatment on the tension-compression yield symmetry. Thus, the objectives of this part of the dissertation was to identify the cyclic deformation behavior of extruded GW103K alloy subjected to varying heat treatment procedures, and determine the fatigue life under varying strain amplitudes, with particular attention to the effect of the RE element addition on the twin formation and twin-particle interaction.
\end{abstract}




\subsection{Microstructure}

\subsubsection{As-extruded GW103K alloy}

Fig. 6.1 shows typical optical micrographs and SEM back-scattered electron images of GW103K alloy in different states (as-extruded, T5, and T6). It is seen from Fig. 6.1(a) that uniform equiaxed grains with an average grain size of about $12 \mu \mathrm{m}$ were obtained in asextruded sample due to the occurrence of dynamic recrystallization (DRX) in the hot extrusion process at $400^{\circ} \mathrm{C}$. As reported earlier in Chapter 4, the grain size of as-extruded sample was fairly small in comparison with the common extruded $\mathrm{Mg}$ alloys, such as AZ31 and AM30 [25-28,35,36,97,108]. Fig. 6.1(b) shows a typical SEM back-scattered electron image of as-extruded sample where several RE containing particles can be seen. By means of EDS point analysis, the content at points A, B, and C of Fig. 6.1(b) was estimated to be 57.8\% $\mathrm{Mg}-17.5 \% \mathrm{Y}-22.5 \% \mathrm{Gd}-2.2 \% \mathrm{Zr}, 79.3 \% \mathrm{Mg}-5.2 \% \mathrm{Y}-15.0 \% \mathrm{Gd}-0.5 \% \mathrm{Zr}$, and $84.9 \% \mathrm{Mg}-3.1 \%$ Y-11.7\% Gd-0.3\% Zr (in wt.\%), respectively. A similar type of microstructures was also reported in the RE containing extruded $\mathrm{Mg}$ alloys in the literature, e.g., Mg-11.90Gd-0.81Y0.44Zr alloy by Zhang et al. [113], Mg-10Gd-3Y-0.5Zr by Liu et al. [212], and Mg-10Gd3Y-0.5Zr and Mg-10Gd-3Y-1.0Zn-0.5Zr alloys by Liu et al. [87]. In addition, EDS line scan was performed as shown in Fig. 6.2(a) and Fig. 6.2(b) which also confirmed the presence of RE-rich particles in the as-extruded alloy, where all the regular (squared, rectangular, etc.) shaped particles contained all three major alloying elements of Gd, Y, and Zr. 


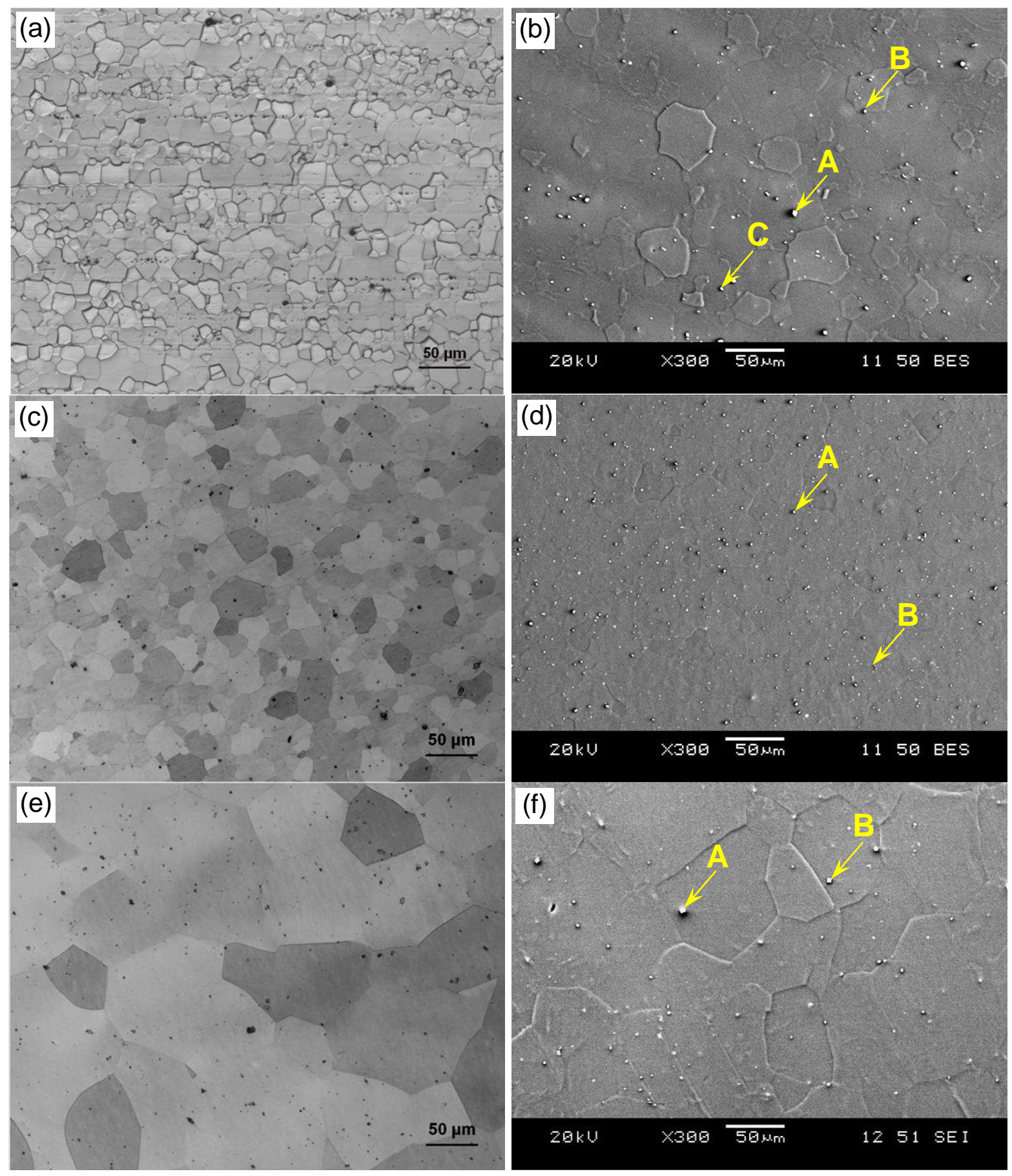

Figure 6.1: Microstructures (left optical micrographs and right SEM back-scattered electron images) of the GW103K alloy in the (a) and (b) as-extruded, (c) and (d) T5, and (e) and (f) T6 states. 

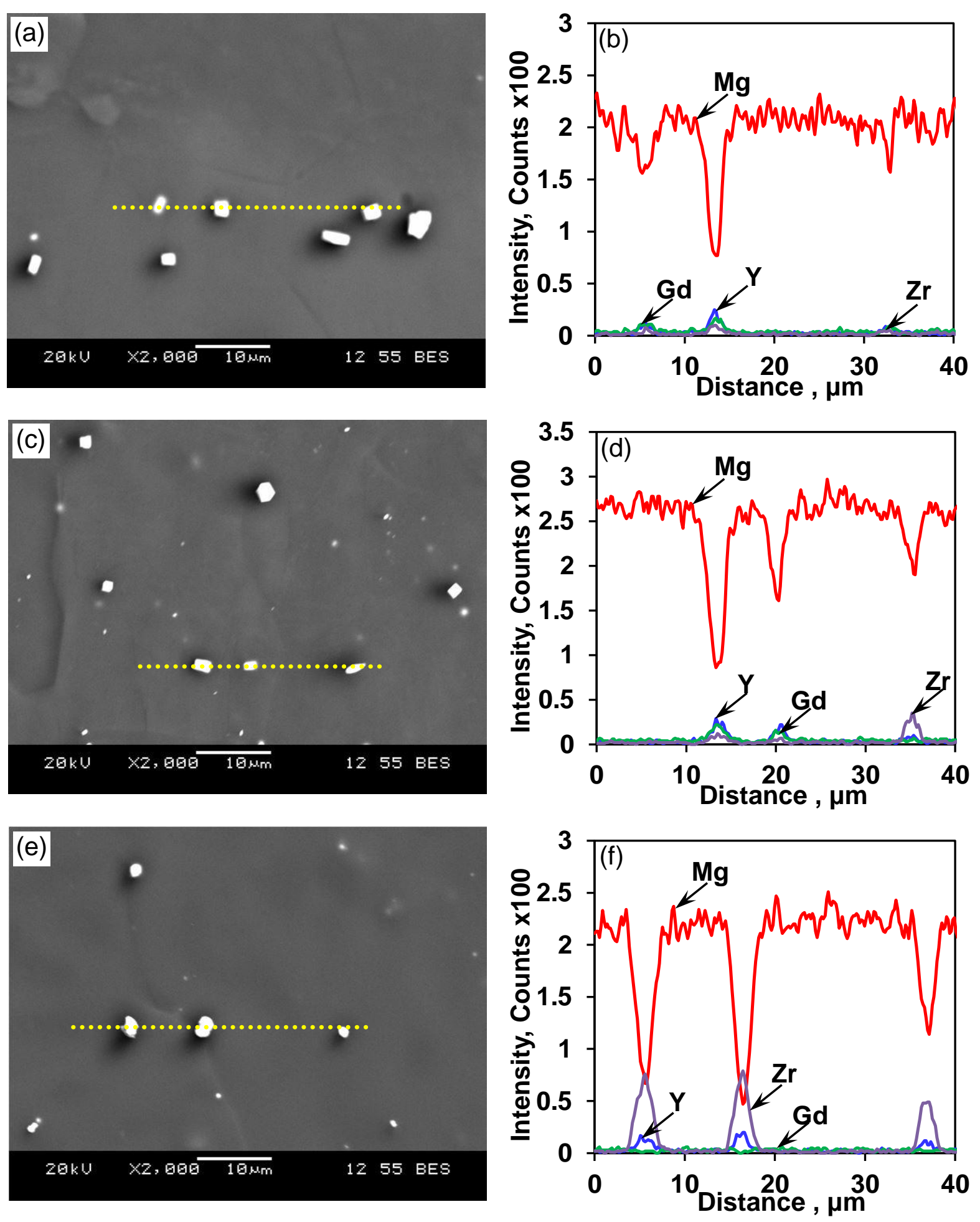

Figure 6.2: SEM back-scattered electron images indicating EDS line scan positions and the corresponding EDS line scan results of GW103K alloy the (a) and (b) as-extruded, (c) and (d) T5, and (e) and (f) T6 conditions. 


\subsubsection{T5 heat treated GW103K alloy}

The optical and SEM back-scattered electron images of T5 alloy are shown in Fig. 6.1(c) and Fig. 6.1(d). The average grain size of the T5 alloy was about $25 \mu \mathrm{m}$ which was almost doubled in comparison with the as-extruded alloy, which indicates grain growth in the aging process at $225^{\circ} \mathrm{C}$ for $16 \mathrm{~h}$. Similar to the as-extruded alloy, RE-rich particles can also be seen from Fig. 6.1(d). By means of EDS point analysis, the contents at points A and B of Fig. 6.1(d) were estimated to be $69.2 \% \mathrm{Mg}-17.3 \% \mathrm{Gd}-13.2 \% \mathrm{Y}$, and $88.0 \% \mathrm{Mg}-1.2 \% \mathrm{Y}-9.7 \%$ Gd-1.2\% $\mathrm{Zr}$ (in wt.\%), respectively. It is clear from the point analysis that the particles in the T5 alloy had more or less the same composition as those in the as-extruded alloy. The EDS line scan was also conducted to confirm the presence of RE-rich particles as shown in Fig. 6.2(c) and Fig. 6.2(d). However, as seen from Fig. 6.2(d) the intensity of Zr-rich particles (irregular shaped particles as shown in Fig. 6.2(c)) seemed to be higher as compared to the regular RE-rich particles in the T5 alloy due to the aging process. Wang et al. [213] also reported that the square-shaped second phase particles were mostly rich with Gd and Y and round-shaped second phase particles were rich with $\mathrm{Zr}$, which actually concurred with the results of the GW103K alloy in both as-extruded and T5 states.

\subsubsection{T6 heat treated GW103K alloy}

Fig. 6.1(e) and (f) show the microstructures of T6 alloy. As shown in Fig. 6.1(e), the average grain size became about $92 \mu \mathrm{m}$ which was almost four times larger than that in the T5 alloy, which indicates a marked grain growth due to the solution treatment at $500^{\circ} \mathrm{C}$ for $2 \mathrm{~h}$. Similar 
grain growth has been reported for Mg-10Gd-3Y alloy in the T4 and T6 conditions by Dong et al. [102] and Liu et al. [103]. It can also be seen from Fig. 6.1(f) that relatively fewer particles were present in the T6 state as compared with the as-extruded and T5 states. The contents at points A and B of Fig. 6.1(f) were estimated to be $12.8 \% \mathrm{Mg}-45.8 \% \mathrm{Gd}-40.5 \%$ Y-1\% Zr, and $67.7 \% \mathrm{Mg}-12.3 \% \mathrm{Y}-18.7 \% \mathrm{Gd}-1.3 \% \mathrm{Zr}$ (in wt.\%), respectively. The EDS line scan results (Fig. 6.2(e) and Fig. 6.2(f)) shows that mainly round shaped particles containing more $\mathrm{Zr}$ appeared in the $\mathrm{T} 6$ state. Similar observations were also reported by Wang et al. [213]. Based on the Mg-Gd phase diagram [69,214] and Mg-Y phase diagram [215], only one single phase solid solution should appear in the Mg-10Gd-3Y-0.5Zr (GW103K) alloy during solution treatment at $500^{\circ} \mathrm{C}$. It was reported that the solubility of $\mathrm{Gd}$ and $\mathrm{Y}$ in $\mathrm{Mg}$ at $500^{\circ} \mathrm{C}$ was about $20 \mathrm{wt} . \%$ and $10 \mathrm{wt} . \%$, respectively $[69,215]$, whereas the solubility of $\mathrm{Zr}$ in Mg was very limited [216]. This suggests that most of the Gd and Y particles could be dissolved into the matrix during the solution treatment at $500^{\circ} \mathrm{C}$ for $2 \mathrm{~h}$ as seen from Fig. 6.2(f), leaving a relatively high Zr-rich particles (round shaped particles in Fig. 6.2(e)) and more discernible $\mathrm{Zr}$ peaks (Fig. 6.2(f)). In addition, RE-enrichment particles were also identified by three zone-axis electron microdiffraction patterns via TEM in the T6 alloy, as shown in Fig. 6.3. Electron microdiffration pattern in Fig. 6.3(b) indicates the structure of particle A in Fig. 6.3(a) which was identified as a cuboid-shaped (RE-rich) particle (facecentered cubic, fcc with $a=0.56 \mathrm{~nm}$ ). Similar results were reported in the cast-T6 samples of Mg-10Gd-2Y-0.5Zr alloy by He et al. [57], cast T6 samples of Mg-4Y-2Nd-1Gd-0.4Zr alloy by Liu et al. [107], and Mg-9Gd-2Er-0.4Zr alloy after solution treatment by Wang et al. [217]. Fig. 6.3(c) and Fig. 6.3(d) show the TEM micrographs recorded from the sample after peak-aging at $225^{\circ} \mathrm{C}$ for $16 \mathrm{~h}$, with the incident electron beam approximately parallel to 
$[0001]_{\alpha}$. A large number or high density of bamboo leaf/plate shaped precipitates with a diameter less than $15 \mathrm{~nm}$ formed and distributed uniformly in the matrix, as shown in Fig. 6.3(c). Such precipitates were identified by selected area electron diffraction (SAED) analysis to be a $\beta^{\prime}$ phase which had a $c$-based centered orthorhombic structure (cbco) $\left(a=2 \times a_{\alpha-\mathrm{Mg}}=0.64 \mathrm{~nm}, b=8 \times d(10 \overline{1} 0)_{\alpha-\mathrm{Mg}}=2.22 \mathrm{~nm}, c=c_{\alpha-\mathrm{Mg}}=0.52 \mathrm{~nm}\right)[57,76,104,118,119]$.
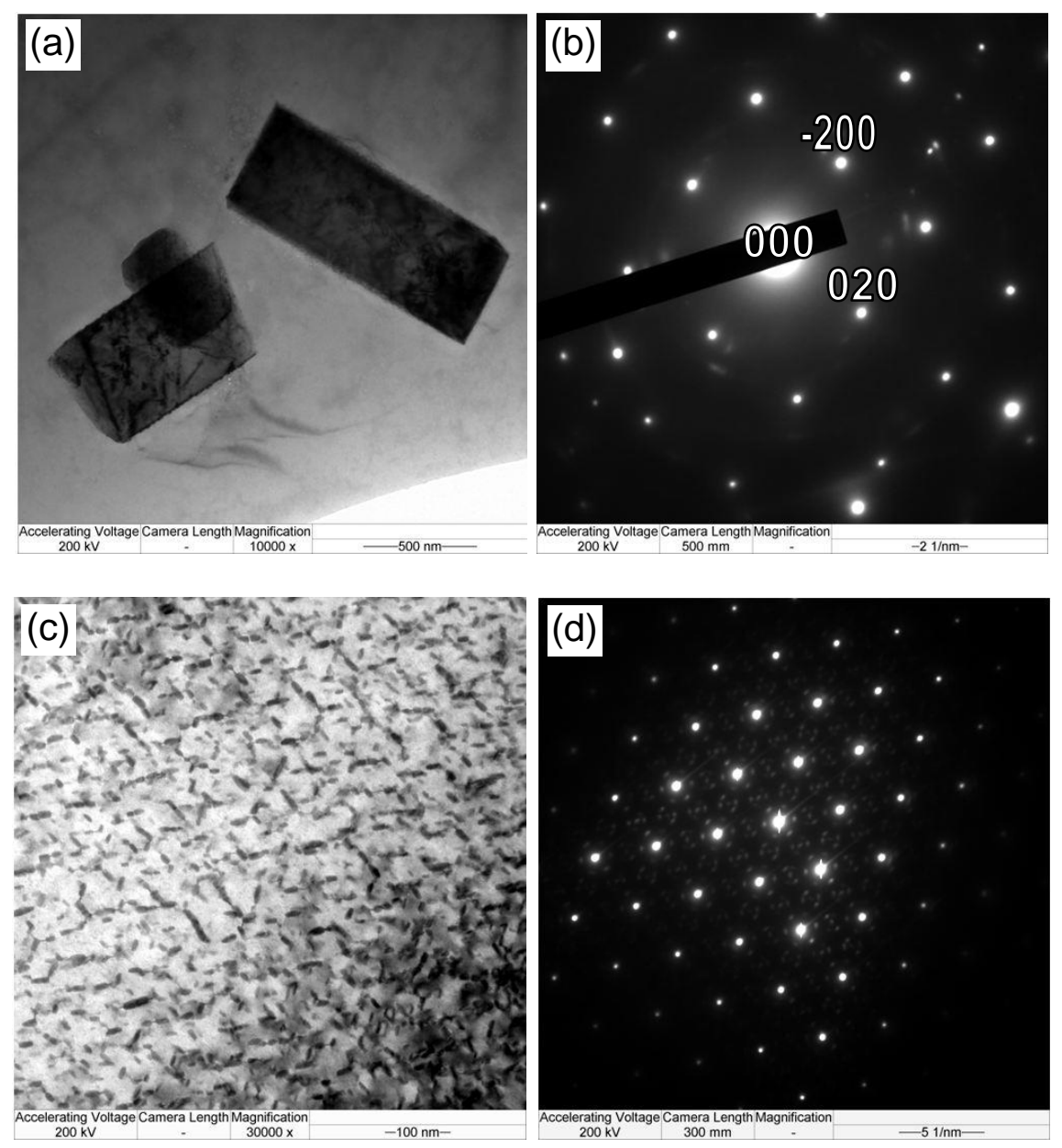

Figure 6.3: Transmission electron micrographs recorded from a sample of T6 alloy: (a) bright-field image taken along [001] zone axis of particle A, (b) [001] zone axis microdiffraction pattern recorded from particle A, (c) bright-field images taken along

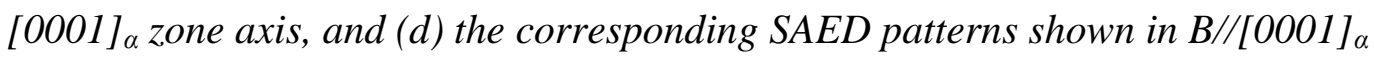


The composition of $\beta^{\prime}$ phase was determined to be approximately $\mathrm{Mg}_{7}(\mathrm{Gd}, \mathrm{Y})$ as reported by Gao et al. [218] and Nishijima et al. [219]. A similar type of precipitates has also been reported in the cast $\mathrm{T} 6$ samples of $\mathrm{Mg}-10 \mathrm{Gd}-2 \mathrm{Y}-0.5 \mathrm{Zr}$ alloy by $\mathrm{He}$ et al. [57], solutiontreated and aged Mg-10Gd-3Y-0.4Zr alloy by He et al. [104], and solution-treated and aged Mg-15Gd-0.5Zr alloy by Gao et al. [76].

\subsection{Crystallographic textures}

Fig. 6.4 shows the crystallographic textures (basal (0001), prismatic (10 $\overline{1} 0)$, and pyramidal (10 $\overline{1} 1$ ) pole figures) of the GW103K alloy in different states (as-extruded, T5, and T6 conditions) evaluated using MTEX software, where ED stands for the extrusion direction and RD denotes the radial direction. As seen from Fig. 6.4, relatively weaker textures (with a maximum intensity of 2.1 multiples of random distribution (MRD) for the as-extruded, 3.3 MRD for the T5, and 4.5 MRD for the T6 samples were observed after the de-focusing correction, in comparison with the extruded AM30 [36,108] and rolled AZ31 [157]. This was basically in agreement with the study by Liu et al. [103], where the intensity of the T5 and T6 textures was stronger than that of the extruded alloy. The presence of such weaker textures in the RE-Mg alloy was a major benefit of adding RE elements into Mg alloys, as also reported by Stanford and Barnett [60] who observed that microalloying with RE elements could weaken texture in the forming process. It was also reported that an addition of small amount of Gd [115] and Ce [52] to Mg alloys could significantly reduce the texture of the extruded alloy and the addition of $\mathrm{Y}$ element had a similar effect on the texture of $\mathrm{Mg}$ 
alloys [121,122]. Furthermore, Bohlen et al. [117] reported that the overall texture intensity and the basal pole intensity aligned with the sheet normal direction were lower in the REcontaining alloys than in the conventional alloys. Similar weaker texture in the Mg-based alloys with the addition of RE elements was also reported in [37,42,45-49,117].

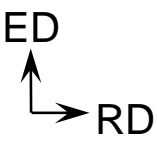

(a) As-extruded

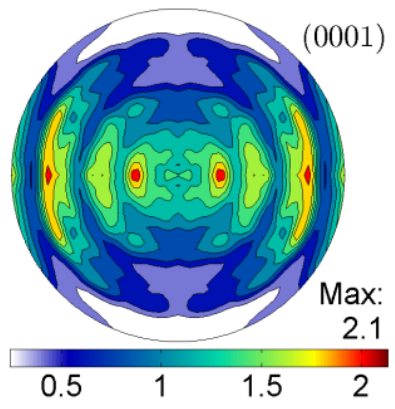

Prismatic

plane

$(10 \overline{1} 0)$

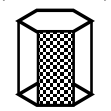

Pyramidal plane $(10 \overline{1} 1)$
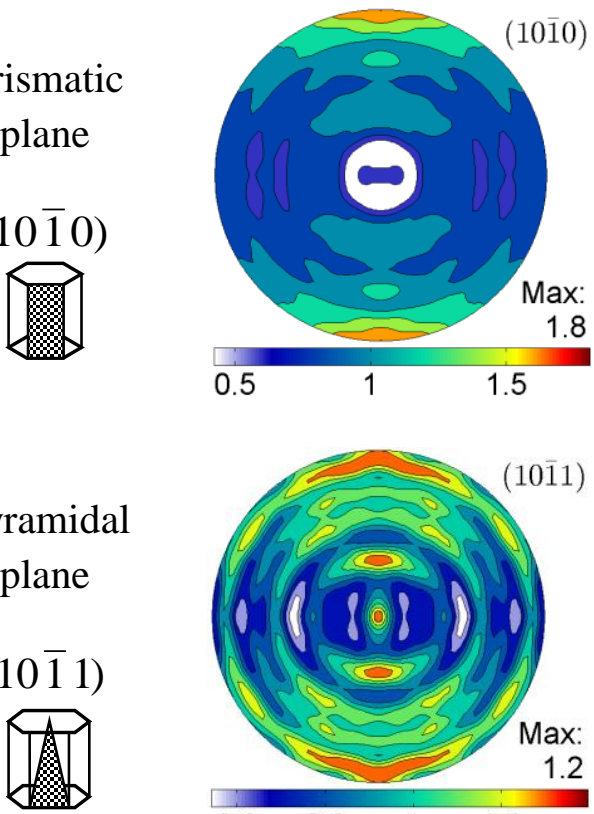

$(10 \overline{1} 1)$ (b) $\mathrm{T} 5$
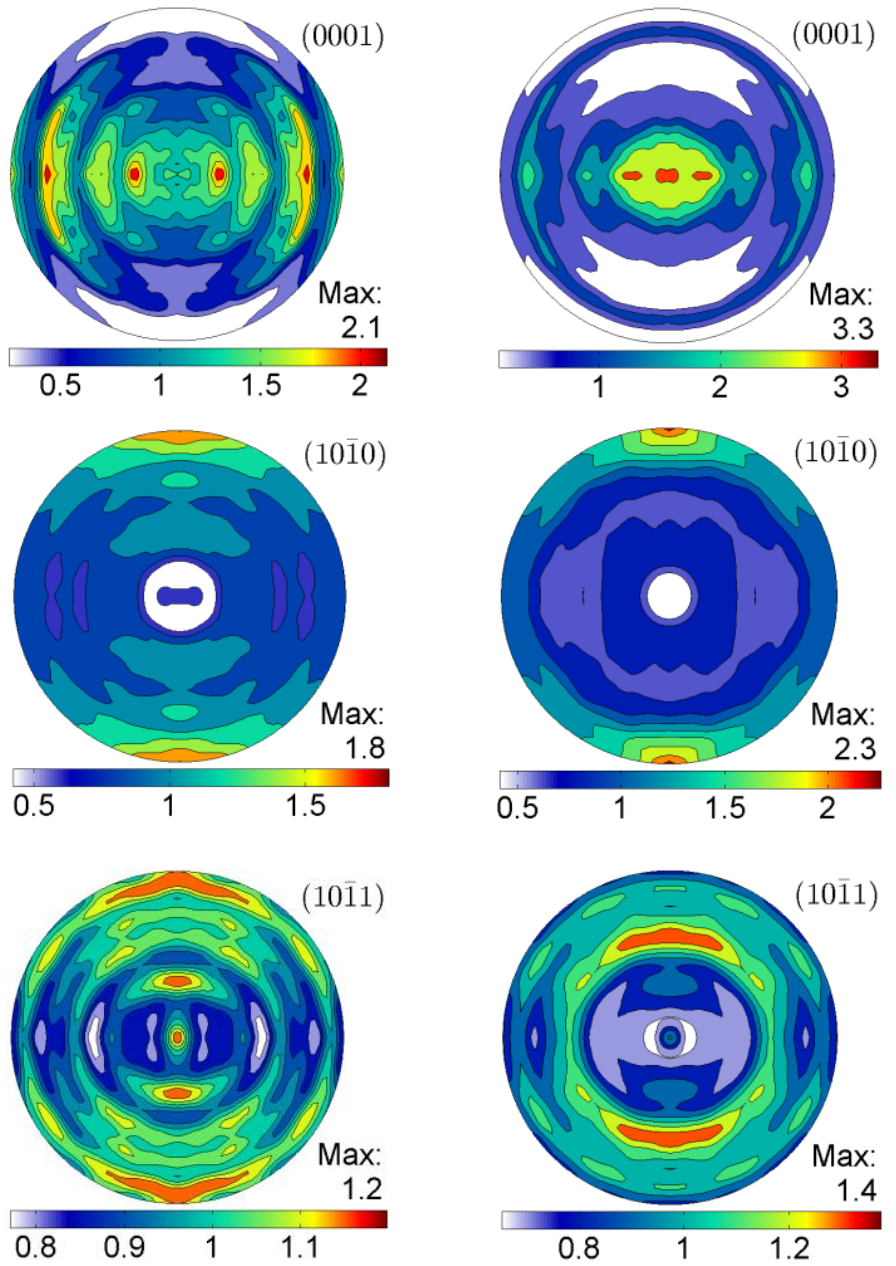

(c) T6
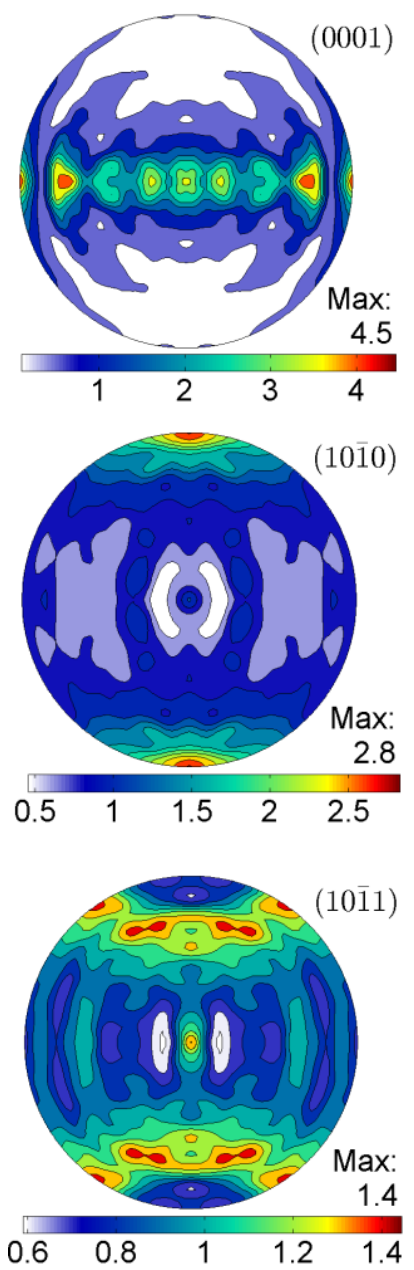

Figure 6.4: Pole figures of basal (0001) plane, prismatic (10 $\overline{1} 0)$ plane, and pyramidal (1011) plane of GW103K alloy in the (a) as-extruded, (b) T5, and (c) T6 states, where ED indicates the extrusion direction and $R D$ denotes the radial direction. 
In the as-extruded sample (Fig. 6.4(a)), it can be seen that the $c$-axes of hcp unit cells in most grains were oriented towards the radial direction (RD), as indicated by the basal (0001) pole distribution, along with the prismatic (10 $\overline{1} 0$ ) and pyramidal (10 $\overline{1} 1)$ poles towards the extrusion direction (ED). In the T5 sample (Fig. 6.4(b)), while the $c$-axes of most grains were oriented towards the RD, a slight change of the basal (0001) pole has been observed, i.e., the basal (0001) pole intensity at the center became higher. It is also seen from the Fig. 6.4(b) that similar to the as-extruded sample (Fig. 6.4(a)), the prismatic (10 $\overline{1} 0$ ) and pyramidal (101 1) poles towards the ED, which indicates that the textures of T5 samples basically tried to retain during the aging process and the components seemed elongated in the RD. This elongation was more expected in the RE containing alloy rather than in the standard alloy AZ31 [48]. Similar tendency of retaining the texture pattern has been reported for Mg-10Gd3Y Mg alloy after $\mathrm{T} 5$ aging treatment at $225^{\circ} \mathrm{C}$ for 4,10 , and $150 \mathrm{~h}$ by Liu et al. [103], for cast $\mathrm{Mg}-1 \mathrm{Zn}-0.3 \mathrm{Ce}$ (ZE10) alloy after annealing treatment at $400^{\circ} \mathrm{C}$ for $15 \mathrm{~min}$ by Machenzie and Pekguleryuz [220], and for as-extruded AZ31 alloy after annealing treatment at $450^{\circ} \mathrm{C}$ and $520^{\circ} \mathrm{C}$ for $30 \mathrm{~min}, 3 \mathrm{~h}$, and $17 \mathrm{~h}$ by Perez-Prado and Ruano [221]. The textures in the T6 sample (Fig. 6.4(c)) exhibited a greater spread of basal (0001) pole around the RD as compared with other two alloys (as-extruded and T5). However, the intensity variation of this T5 alloy was in contrast to that of Liu et al. [103]. Hirsch and Al-Samman [42] also reported that the texture weakening occurred during recrystallization annealing. This difference was mainly due to the combined effects of RE elements and the precipitates formed during the heat treatment. 


\subsection{Tensile properties}

Typical tensile stress-strain curves of the GW103K alloy in different states tested at a strain rate of $1 \times 10^{-4} \mathrm{~s}^{-1}$ is shown in Fig. 6.5 and the tensile properties obtained are listed in Table 6.1. As seen from Table 6.1, the GW103K alloy (as-extruded, T5, and T6) exhibited a higher tensile yield strength (YS) than the RE-free AM30 alloy, i.e., 232 MPa, 332, and $342 \mathrm{MPa}$ vs. $189 \mathrm{MPa}$ as reported in [28]. This indicated an obvious beneficial role of RE element addition in the Mg alloy, which was in agreement with those in an extruded Mg-8Al-xRE alloy [222], where the improved tensile properties due to the addition of RE elements were reported as well. As seen from Fig. 6.5 and Table 6.1, in comparison with the as-extruded alloy, significant improvements in the YS and ultimate tensile strength (UTS) were attained in the T5 alloy, by approximately $100 \mathrm{MPa}$ and $108 \mathrm{MPa}$, respectively. Similar results were reported in the T5 samples of Mg-10Gd-2Y-0.5Zr alloy by He et al. [57], T5 samples of Mg10Gd-3Y alloy by Liu et al. [103], T5 samples of Mg-10Gd-3Y-0.5Zr alloy by Liu et al. [87], and extruded samples of Mg-10Gd-3Y-0.5Zr alloy by Yang et al. [37], where marked improvement in the YS and UTS was seen after the T5 aging treatment as well. However, the percent elongation of the T5 alloy (6.6\%) decreased by about $20 \%$ as compared with that of the as-extruded alloy (8.3\%). These results were due to the precipitation hardening as seen in Fig. 6.3 and also reported in [57,107]. As seen from Table 6.1, a further improvement in the YS (about $110 \mathrm{MPa}$ ) and UTS (about $112 \mathrm{MPa}$ ) was achieved via the T6 heat treatment as compared with the as-extruded alloy. Similar improvements in the YS and UTS in the T6 alloy have been reported by Liu et al. [107]. The percent elongation of the T6 alloy decreased more as compared with that of the as-extruded alloy. The relatively small difference between 
the T5 and T6 states, as seen from Fig. 6.5 and Table 6.1, was a consequence of a combined role of grain size and precipitates. While the T6 heat treatment would lead to a more complete precipitation hardening due to the formation of the supersaturated solid solution in the solution treatment phase prior to aging, the much larger grain size (Fig. 6.1(e) and (f)) due to the same solution treatment caused a certain extent of sacrifice or cutback in the strength based on the well-known Hall-Petch relationship [34] and in the ductility as well.

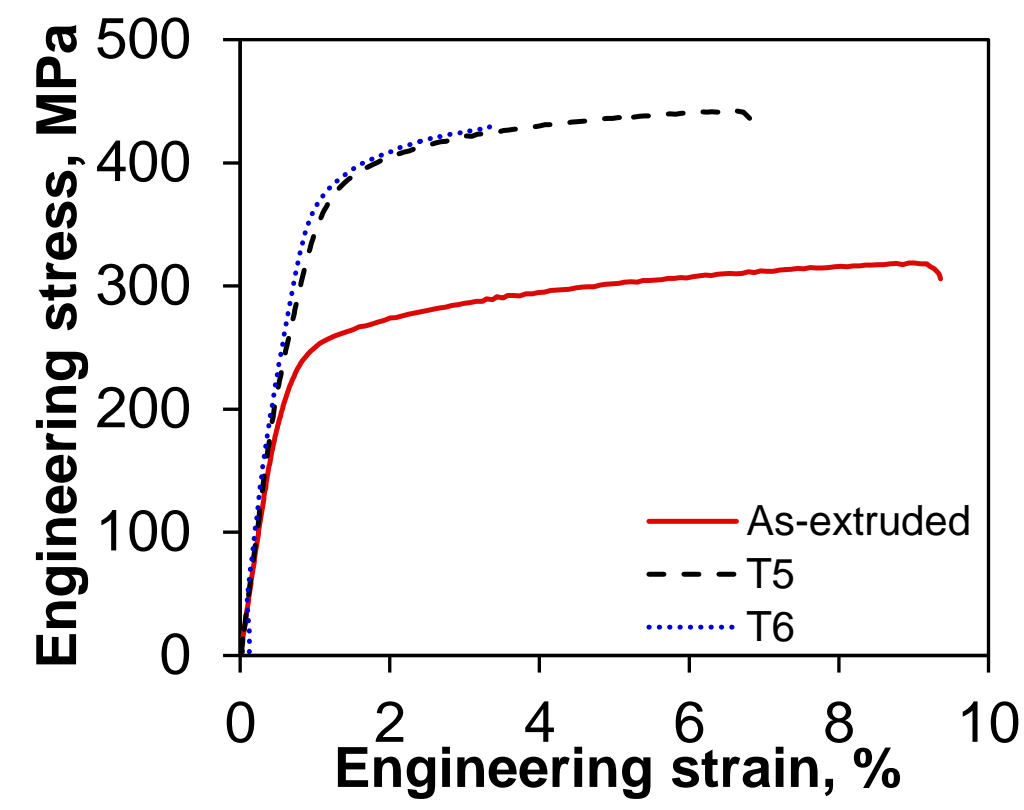

Figure 6.5: Typical tensile stress-strain curves of the GW103K alloy in different conditions tested at a strain rate of $1 \times 10^{-4} \mathrm{~s}^{-1}$.

Table 6.1: Tensile properties of GW103K alloy in different states obtained at a strain rate of $1 \times 10^{-4} \mathrm{~s}^{-1}$

\begin{tabular}{ccccc}
\hline Materials & $\sigma_{\mathrm{YS}}, \mathrm{MPa}$ & $\sigma_{\mathrm{UTS}}, \mathrm{MPa}$ & Elongation, \% & $n$ \\
\hline As-extruded & 232 & 318 & 8.3 & 0.15 \\
T5 & 332 & 426 & 6.6 & 0.19 \\
T6 & 342 & 430 & 3.2 & 0.17 \\
\hline
\end{tabular}




\subsection{Low cycle fatigue properties}

\subsubsection{Hysteresis loops}

Fig. 6.6 shows typical stress-strain hysteresis loops of the first, second, and mid-life cycles at a total strain amplitude of $1.2 \%$ and strain ratio of $R_{\varepsilon}=-1$ for the GW103K alloy in different states. It is seen from Fig. 6.6(a) that, like the tensile stress-strain curves (Fig. 6.5), the initial slope in the ascending phase of the first cycle was the same in all the three alloy states, indicating that the T5 and T6 heat treatment did not affect the Young's modulus of the alloy. As normally anticipated, heat treatment would alter the strength of a material, but had little influence on the Young's modulus, since the magnitude of Young's modulus is a measure of the resistance to separation of adjacent atoms, i.e., interatomic bonding forces on an atomic scale. The present GW103K alloy was observed to exhibit nearly symmetrical hysteresis loops in all the three alloy conditions, which were somewhat similar to those of face-centered cubic (fcc) metals (e.g., Al, $\mathrm{Cu}, \mathrm{Ni}$ ) as a result of the dislocation slip-dominated deformation in most materials [33]. This was, however, in sharp contrast to the hysteresis loops in the extruded or rolled $\mathrm{Mg}$ alloys due to the presence of strong crystallographic texture [25-28]. The maximum and minimum peak stresses in the T5 and T6 conditions showed a significant enhancement in different loops as compared with those in the as-extruded GW103K alloy, due to the presence of a large number of precipitates after the T5 and T6 heat treatment (Fig. 6.3). Similar to the tensile properties shown in Fig. 6.5 and Table 6.1, the T6 alloy exhibited only a slightly higher maximum and minimum peak stresses than the T5 alloy (Fig. 6.6). Unlike the fcc metals where the slope of hysteresis loops after the strain reversal either at the 
maximum or minimum stress was basically equal to the value of Young's modulus, the pseudoelastic or nonlinear elastic behavior in both descending and ascending phases in the RE containing GW103K alloy was still in existence, in spite of the symmetrical hysteresis loops. It seemed reasonable to consider that the pseudoelastic behavior would be an inherent characteristic of $\mathrm{Mg}$ alloys, which was related to the superior damping capacity. Further studies in this aspect are needed. However, in comparison with the as-extruded Mg alloy [2528], the pseudoelastic behavior reduced to a certain extent in the GW103K alloy, especially in the T5 and T6 conditions.

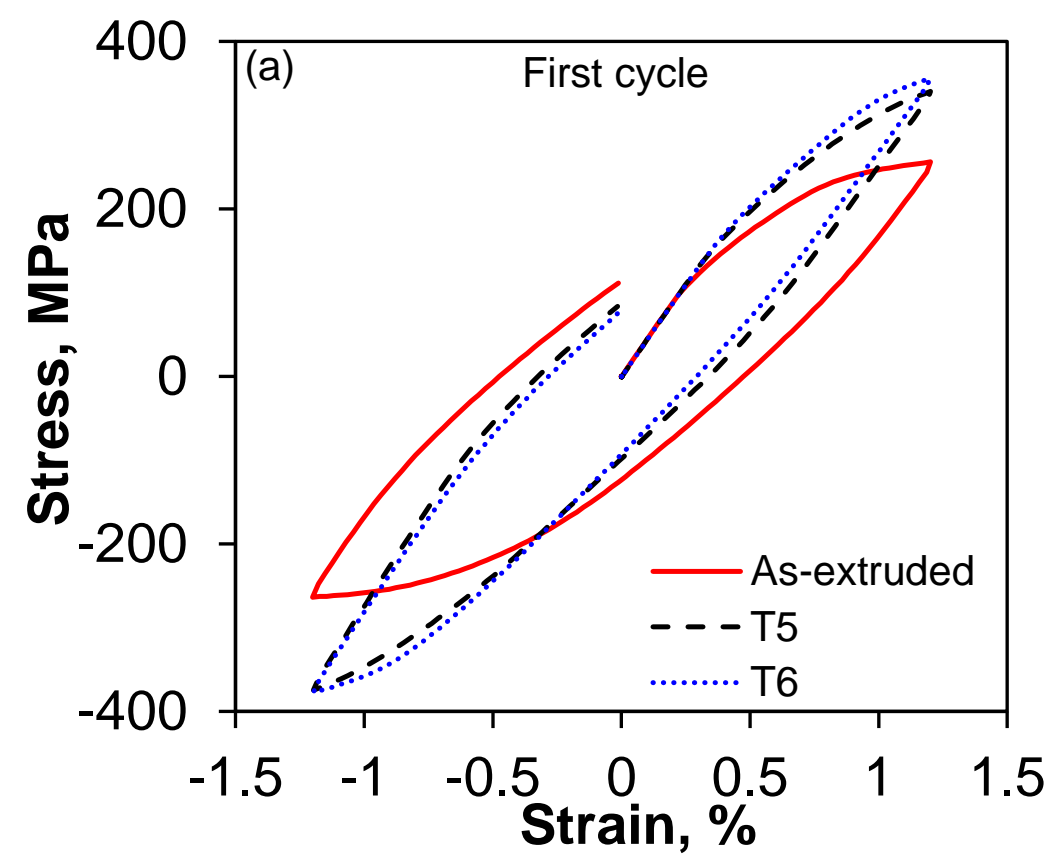



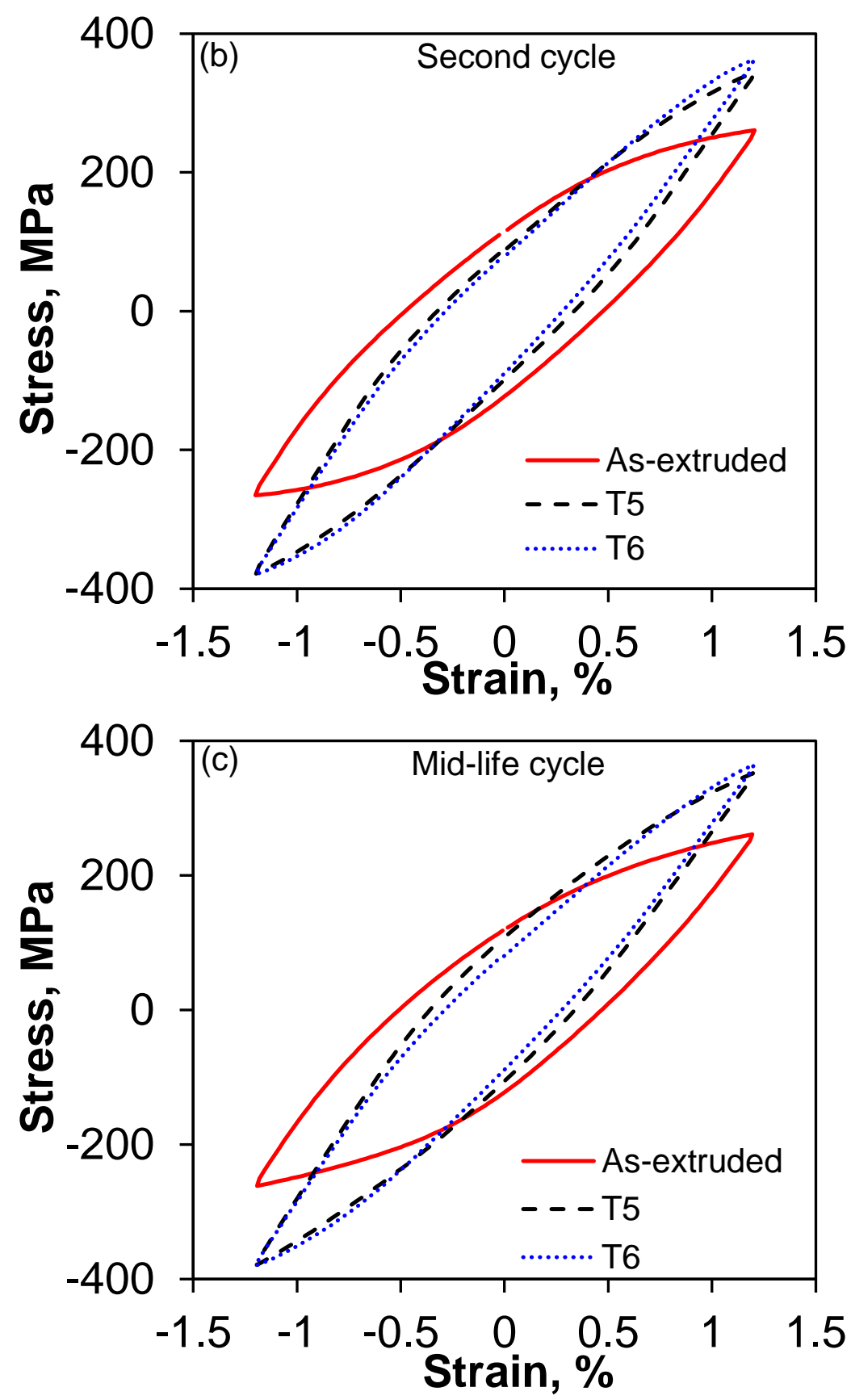

Figure 6.6: Typical stress-strain hysteresis loops of GW103K alloy in different states tested at a given total strain amplitude of $1.2 \%$ and strain ratio of $R_{\varepsilon}=-1$ of (a) first cycle, (b) second cycle, and (c) mid-life cycle. 


\subsubsection{Cyclic deformation response}

Fig. 6.7 shows the evolution of cyclic stress amplitude as a function of the number of cycles at different strain amplitudes on a semi-log scale for the GW103K alloy in the (a) asextruded, (b) T5, and (c) T6 states, respectively. It is seen that as the applied total strain amplitude increased, the cyclic stress amplitude increased and the fatigue life decreased for the GW103K alloy in all states. Unlike the RE-free extruded alloys, e.g., AM30 [28] and AZ31 [25-27] where cyclic stabilization occurred only at lower strain amplitudes of about $0.1 \%$ and $0.2 \%$, the as-extruded GW103K alloy exhibited cyclic stabilization until failure up to a strain amplitude of $1.0 \%$. Even at a strain amplitude of $1.2 \%$ for the as-extruded alloy, only slight change, i.e., an initial slight cyclic hardening within the first three cycles and then minor cyclic softening, could be seen from Fig. 6.7(a), which corresponded well to the variation of the plastic strain amplitude $\left(\Delta \varepsilon_{\mathrm{p}} / 2\right)$ during cyclic deformation as shown in Fig. 6.8(a) for different total strain amplitudes. This was due to the addition of RE elements which significantly changed the microstructure (Fig. 6.1 and Fig. 6.3) and weakened the texture (Fig. 6.4). For the heat-treated T5 and T6 states, cyclic stabilization occurred up to a strain amplitude of $0.6 \%$ (Fig. 6.7(b) and 6.7(c)), which followed by the slight cyclic hardening at the higher strain amplitudes (0.8-1.2\%). These also corresponded well to the decreasing plastic strain amplitude (Fig. 6.8(b) and 6.8(c)). However, while the initial cyclic deformation characteristics were similar for the alloy in different states, the cyclic stress amplitudes and also plastic strain amplitudes at a given total strain amplitude applied were different, except at the lower strain amplitudes $(0.2 \%$ and $0.4 \%)$. For example, at a given total strain amplitude of $0.8 \%$, the stress amplitudes were $\sim 240 \mathrm{MPa}, \sim 290 \mathrm{MPa}$, and $\sim 300$ 
MPa for the as-extruded, T5, and T6 samples, respectively (Fig. 6.7). The difference in the stress amplitudes also increased at higher strain amplitudes. For the plastic strain amplitudes, at a given total strain amplitude of $0.8 \%$, the values were $\sim 0.2 \%, \sim 0.165 \%$, and $\sim 0.11 \%$ for the as-extruded, T5, and T6 samples, respectively (Fig. 6.8(a), (b), and (c)).
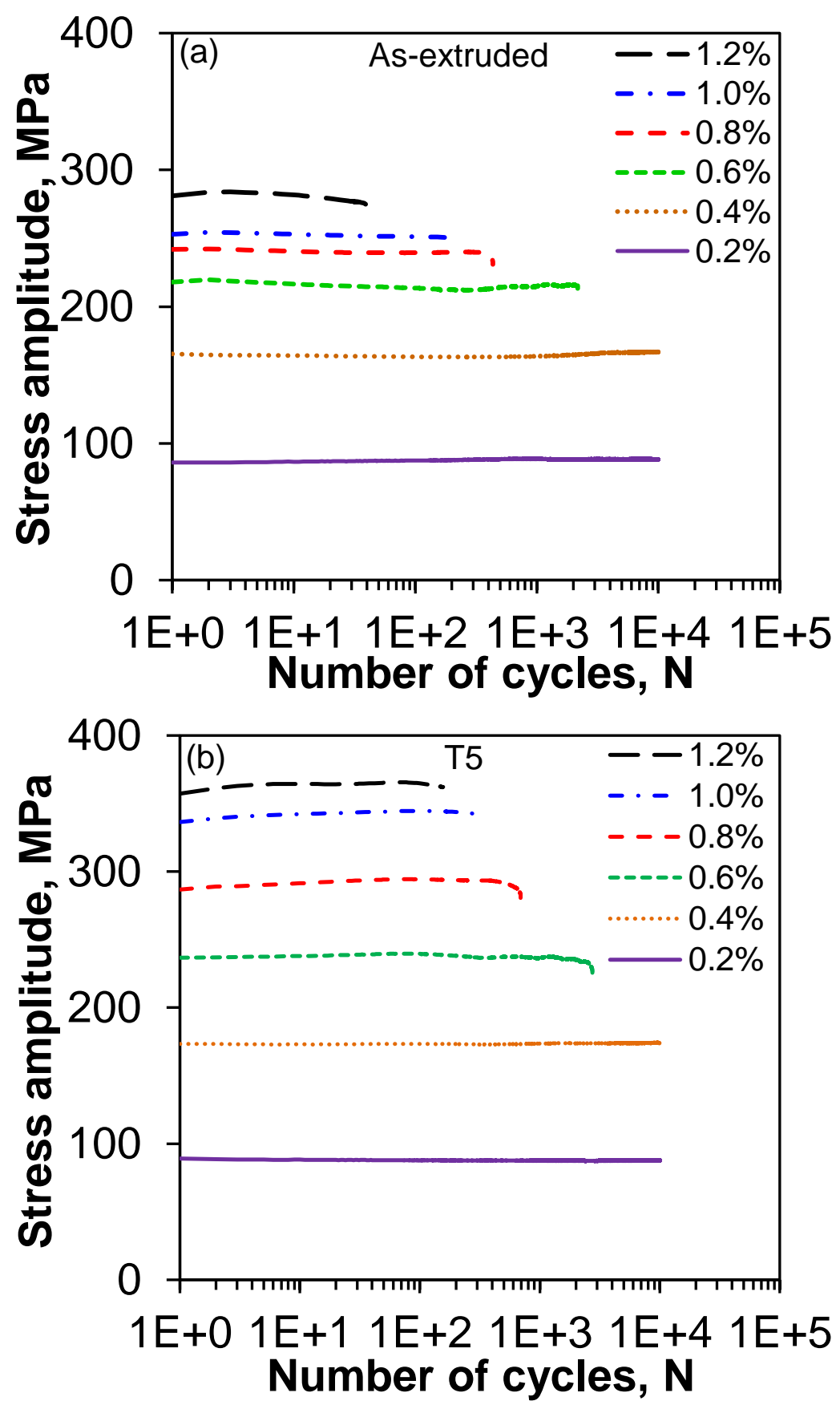


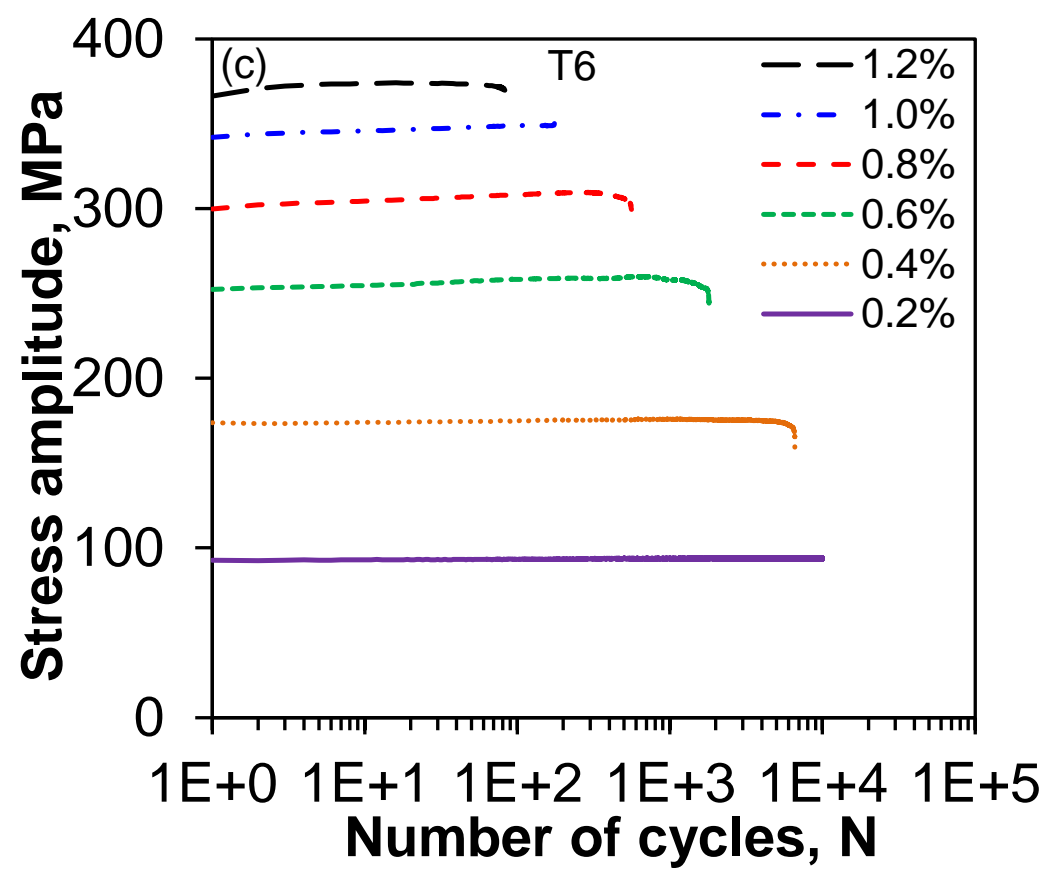

Figure 6.7: Stress amplitude vs. the number of cycles at different total strain amplitudes applied for the GW103K alloy in the (a) as-extruded, (b) T5, and (c) T6 states.

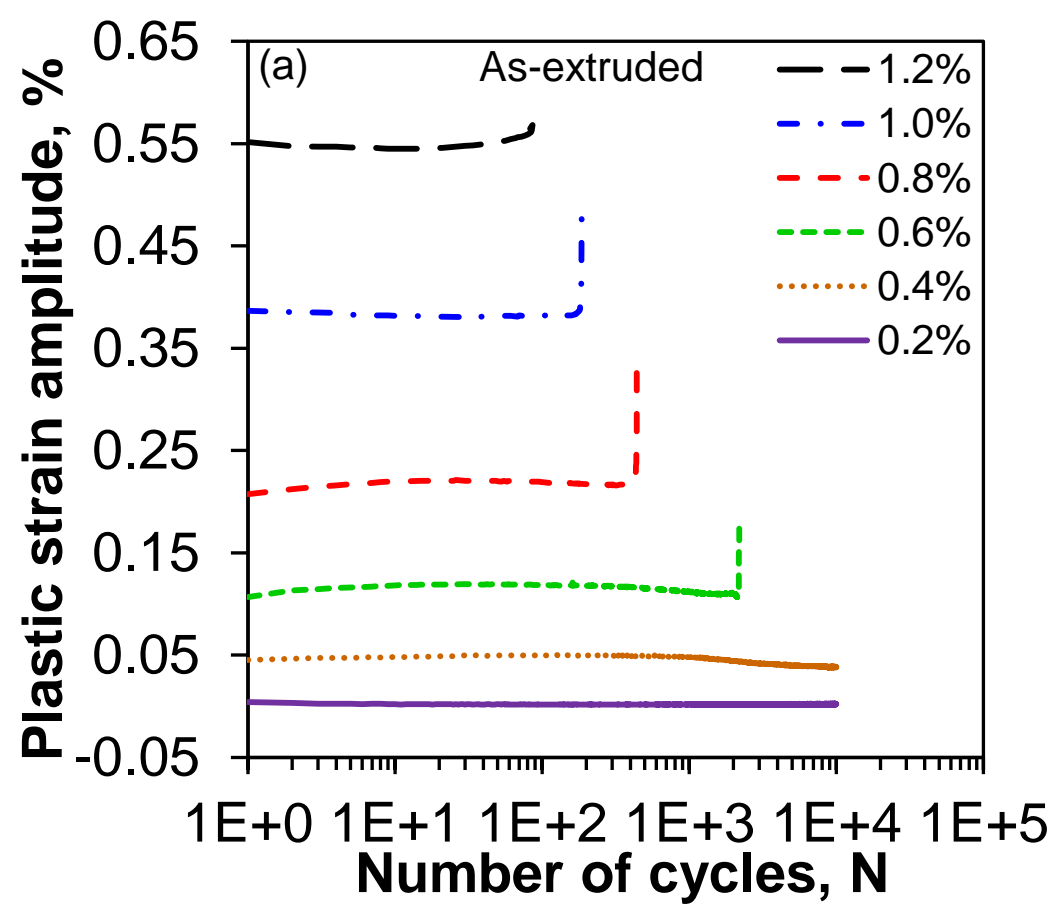



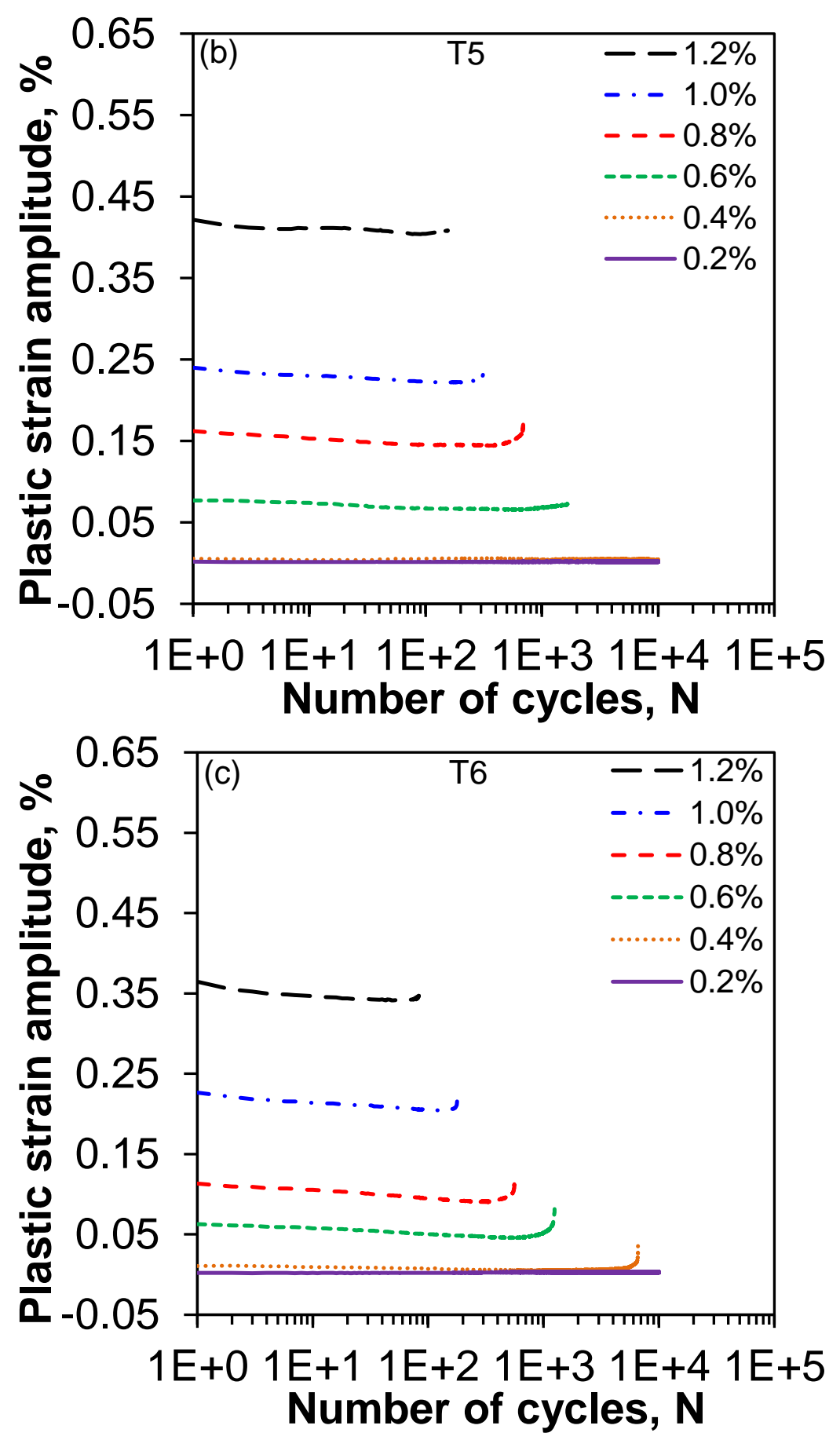

Figure 6.8: Plastic strain amplitude vs. the number of cycles at different total strain amplitudes applied for the GW103K alloy in the (a) as-extruded, (b) T5, and (c) T6 states. 


\subsubsection{Fatigue life and fatigue parameters}

The fatigue life (i.e., the number of cycles to failure, $N_{f}$ ) as a function of the applied total strain amplitudes $\left(\Delta \varepsilon_{\mathrm{t}} / 2\right)$ of the GW103K alloy in the as-extruded, T5, and T6 states is shown in Fig. 6.9, along with the experimental data reported in the literature for various extruded Mg alloys [26,28,156-159] for comparison. The run-out data points were indicated by arrows pointing horizontally at or over $10^{7}$ cycles. The present GW103K alloy showed a trend of increasing fatigue life with decreasing strain amplitude in all conditions and there was no significant difference in the fatigue lives among the different alloy states. Overall, the alloy basically showed an improved fatigue life than the RE-free extruded $\mathrm{Mg}$ alloys [26,28,156-159]. Based on the Basquin equation and Coffin-Manson relation and as described in refs. $[16,25-27,34,134,155]$, the total strain amplitude could be expressed as two parts of elastic strain amplitude and plastic strain amplitude, i.e.,

$$
\frac{\Delta \varepsilon_{t}}{2}=\frac{\Delta \varepsilon_{e}}{2}+\frac{\Delta \varepsilon_{p}}{2}=\frac{\sigma_{f}^{\prime}\left(2 N_{f}\right)^{b}}{E}+\varepsilon_{f}^{\prime}\left(2 N_{f}\right)^{c}
$$

where $E$ is the Young's modulus (for the present alloy the average value obtained during fatigue testing was $\sim 44.5 \mathrm{GPa}$ ), $N_{f}$ is the fatigue life or the number of cycles to failure, $\sigma_{f}^{\prime}$ is

the fatigue strength coefficient, $b$ is the fatigue strength exponent, $\varepsilon_{f}^{\prime}$ is the fatigue ductility coefficient, and $c$ is the fatigue ductility exponent. In addition, cyclic deformation behavior is normally considered to be related to the portion of the plastic strain amplitude and is independent of the elastic strain amplitude, which could be expressed by the following equation [27],

$$
\frac{\Delta \sigma}{2}=K^{\prime}\left(\frac{\Delta \varepsilon_{p}}{2}\right)^{n^{\prime}}
$$


where $\frac{\Delta \sigma}{2}$ is the mid-life stress amplitude, $\frac{\Delta \varepsilon_{p}}{2}$ is the mid-life plastic strain amplitude, $n^{\prime}$ is the cyclic strain-hardening exponent and $K^{\prime}$ is the cyclic strength coefficient. Further improvements in the cyclic strength coefficient $\left(K^{\prime}\right)$ and fatigue strength coefficient $\left(\sigma_{f}^{\prime}\right)$ were seen in the T6 alloy as compared to those of the as-extruded alloy (Table 6.2). The values of fatigue strength exponent $(b)$ and fatigue ductility exponent $(c)$ decreased in both T5 and T6 samples as compared to the as-extruded sample. In general, a smaller absolute value of fatigue strength exponent $(b)$ and fatigue ductility exponent $(c)$ and a larger value of fatigue strength coefficient $\left(\sigma_{f}^{\prime}\right)$ and fatigue ductility coefficient $\left(\varepsilon_{f}^{\prime}\right)$ reflect a longer fatigue life [223].

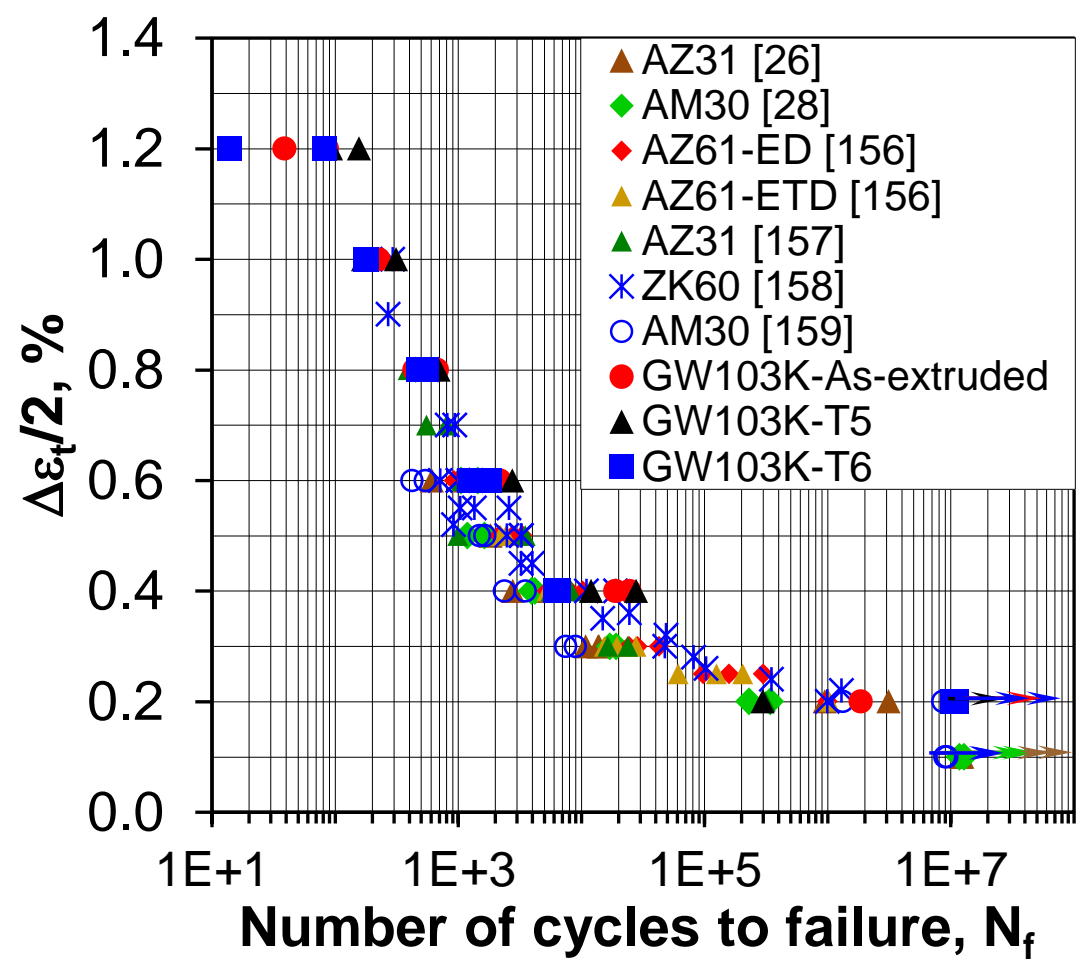

Figure 6.9: Total strain amplitude versus the number of cycles to failure for the GW103K alloy in the as-extruded, T5, and T6 states, in comparison with the data reported in the literature for various extruded Mg alloys [26,28,156-159]. 
This indeed implies that a longer fatigue life of a material in the strain-controlled fatigue tests requires a good combination of both higher strength and superior ductility. In spite of such a seemingly conflicting effect of the exponent pair $\left(b\right.$ and $c$ ) and the coefficient pair ( $\sigma_{f}^{\prime}$ and $\varepsilon_{f}^{\prime}$ ) on the fatigue life, the exponent pair would be expected to play a more significant role in the sense of exponential functions (equ. (6.1)) [223]. In comparison with the as-extruded alloy, the absolute values of $b$ and $c$ in both T5 and T6 states were higher, but the values of $\sigma_{f}^{\prime}$ and $\varepsilon_{f}^{\prime}$ were higher as well. As a result, a coupled role of these fatigue-life prediction parameters listed in Table 6.2 would give an equivalent lifetime of the GW103K alloy in different states within the experimental scatter, as shown in Fig. 6.9, which was in general longer than that of RE-free extruded Mg alloys. It should be noted that in evaluating the above fatigue life parameters, the run-out data for the fatigue samples without failure at or above $10^{7}$ cycles were not included. Furthermore, the obtained cyclic stress-strain curves corresponding to the mid-lives of the GW103K alloy in different conditions are shown in Fig. 6.10 along with the monotonic stress-strain curves. It is seen that cyclic stress-strain curves were generally consistent with the monotonic ones for the GW103K alloy in different conditions within the experimental scatter. The obtained cyclic yield strength $\left(\sigma_{y}^{\prime}\right)$ and cyclic strain hardening exponent $\left(n^{\prime}\right)$ of the GW103K alloy in different states were also equivalent to their respective monotonic yield strength and strain hardening exponent, as seen from Tables 6.1 and 6.2. 
Table 6.2: Low cycle fatigue parameters obtained for the GW103K alloy in the as-extruded, T5, and T6 conditions.

\begin{tabular}{lccc}
\hline Low cycle fatigue parameters & As-extruded & T5 & T6 \\
\hline Cyclic yield strength, $\sigma_{y}^{\prime}, \mathrm{MPa}$ & 215 & 305 & 350 \\
Cyclic strain hardening exponent, $n^{\prime}$ & 0.19 & 0.16 & 0.20 \\
Cyclic strength coefficient, $K^{\prime}, \mathrm{MPa}$ & 734 & 897 & 1230 \\
Fatigue strength coefficient, $\sigma_{f}^{\prime}, \mathrm{MPa}$ & 518 & 811 & 1054 \\
Fatigue strength exponent, $b$ & -0.11 & -0.14 & -0.19 \\
Fatigue ductility coefficient, $\varepsilon_{f}^{\prime}$ & 0.05 & 0.52 & 0.41 \\
Fatigue ductility exponent, $c$ & -0.44 & -0.87 & -0.89 \\
\hline
\end{tabular}

\subsubsection{Fractography}

Fig. 6.11 shows an overall view of fracture surfaces of the GW103K alloy in different conditions at a total strain amplitude of $0.4 \%$, containing fatigue crack initiation, propagation, and final fast fracture regions. It is seen from the low magnification images (Fig. 6.11) that fatigue crack initiated basically from the specimen surface. Compared with the as-extruded and T5 samples, the fracture surfaces of the T6 sample were obviously rougher because of the larger grain size (Fig. 6.1(e) and (f)). Fig. 6.12 shows the fractographs near the crack initiation site and in the crack propagation area of the fatigued samples at higher magnifications. In the near-initiation area (as indicated by the red-dashed box in Fig. 6.11), typical flow lines or tearing ridges indicating fatigue crack growth direction could be seen in both as-extruded and T5 samples as shown in Fig. 6.12(a) and (c), respectively. 

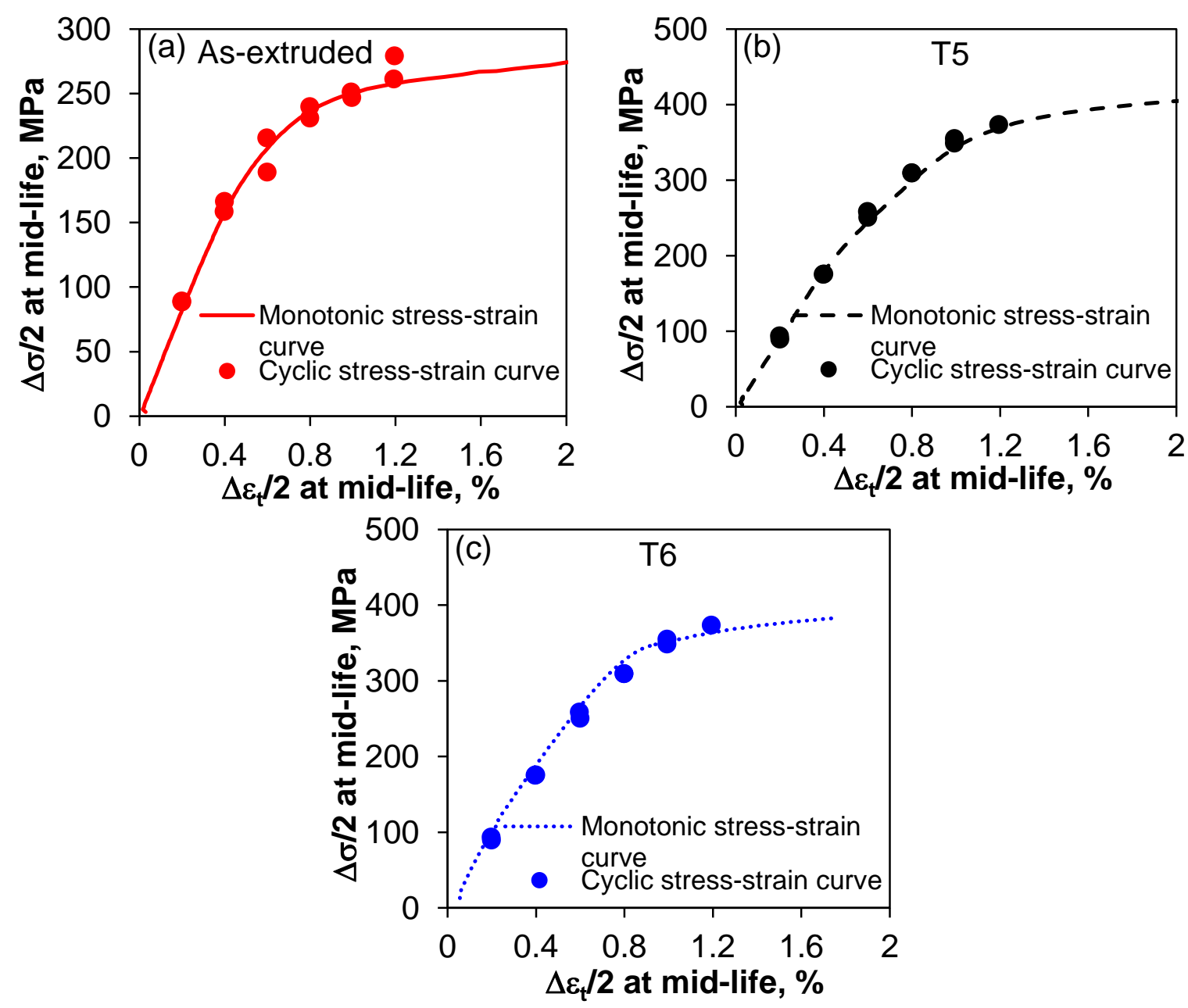

Figure 6.10: Cyclic stress-strain curves for the GW103K alloy in the (a) as-extruded, (b) T5, and (c) T6 states, where the corresponding monotonic stress-strain curves are also plotted for comparison.

Fatigue crack propagation region was basically characterized by the fatigue striations which were perpendicular to the crack propagation direction as shown in Fig. 6.12(b) and (d) for the as-extruded and T5 samples, respectively. However, a mix of cleavage-like features and tearing ridges in the near-initiation area on the fracture surface of the T6 sample fatigued at a strain amplitude of $0.4 \%$ can be seen from Fig. 6.12(e). A three-dimensional image taken in the propagation area is shown in Fig. 6.12(f), with a main feature of fatigue striations as well. 
Similar fracture surface features consisting of a mix of cleavage-like features, tearing ridges, and fatigue striations were reported in the T6 GW103 alloy by Dong et al. [102]. It was also observed that the spacing of fatigue striations became progressively larger with increasing distance from the crack initiation site. Since each fatigue striation could be assumed to roughly represent a single loading cycle, the spacing of fatigue striations could reflect the fatigue crack propagation rate and the associated fatigue life [223]. It is known that the occurrence of fatigue striations was due to a repeated plastic blunting-sharpening process in face-centered cubic (fcc) materials arising from the slip of dislocations in the plastic zone in front of the fatigue crack tip [224]. The formation of the fatigue striations in the hexagonal close-packed (hcp) $\mathrm{Mg}$ alloy was anticipated to be related to both dislocation slip and twinning in the plastic zone during fatigue crack propagation [25-28]. Unlike the as-extruded and T5 samples, crack propagation near initiation in the T6 sample shown in Fig. 6.11(c) and Fig. 6.12(e) appeared fairly different. To make sure where the crack initiation was, two different regions as indicated by the yellow-dashed boxes in Fig. 6.11(c) were examined in more detail and shown in Fig. 6.13. The region close to the upper left corner had fatigue striations coupled with the cleavage-like facets (Fig. 6.13(a) and (b)), while no sign of fatigue striations could be identified in the region near the lower right corner (Fig. 6.13(c) and (d)). Thus, fatigue crack initiation in the T6 sample was confirmed to be from the upper left corner (as indicated by the red-dashed box in Fig. 6.11(c)) rather than from the seemingly flatter surface at the lower right corner. The occurrence of the cleavage-like features was likely to be associated with the much larger grain size (Fig. 6.1(e) and (f)) stemming from the relatively high solution temperature at $500^{\circ} \mathrm{C}$ for $2 \mathrm{~h}$. 


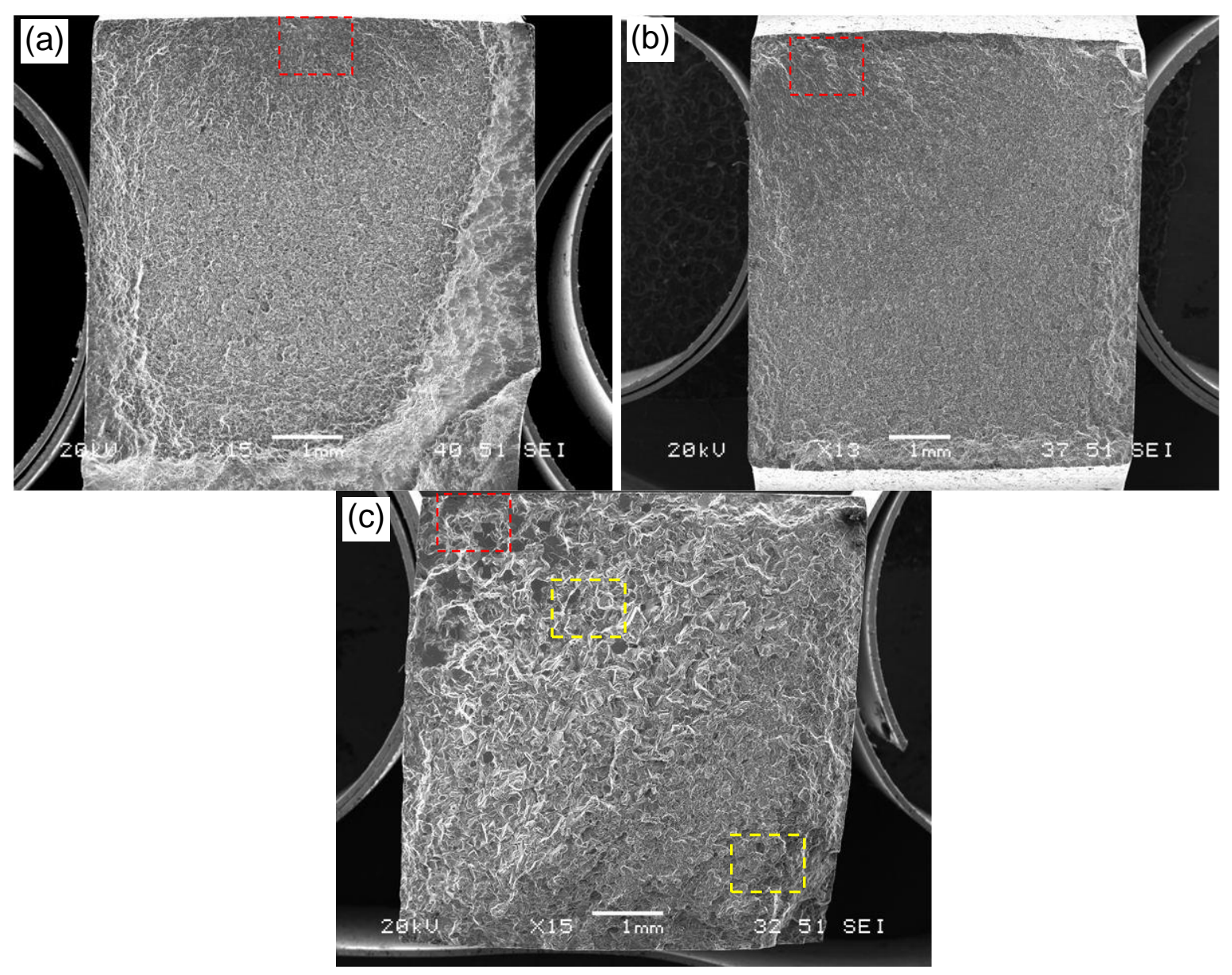

Figure 6.11: An overall view of fracture surfaces fatigued at a strain amplitude of $0.4 \%$ of the GW103K alloy in the (a) as-extruded, (b) T5, and (c) T6 states.
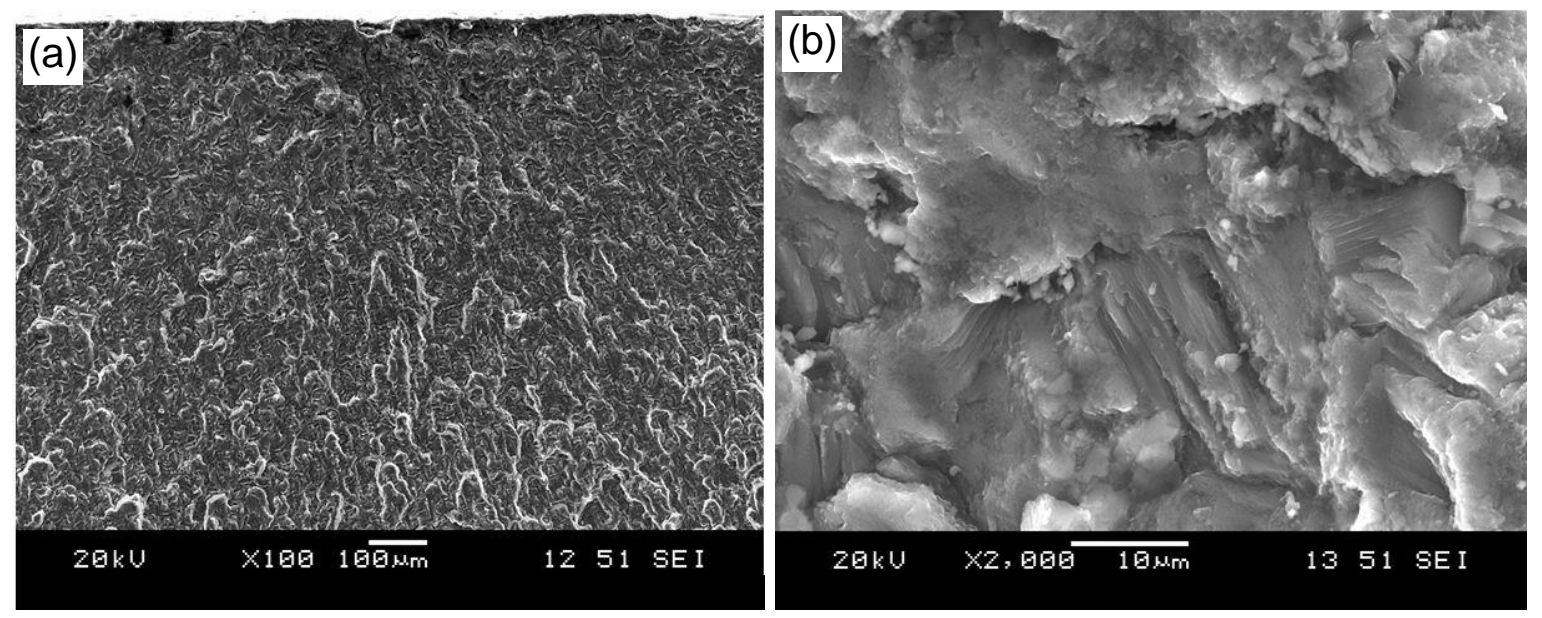

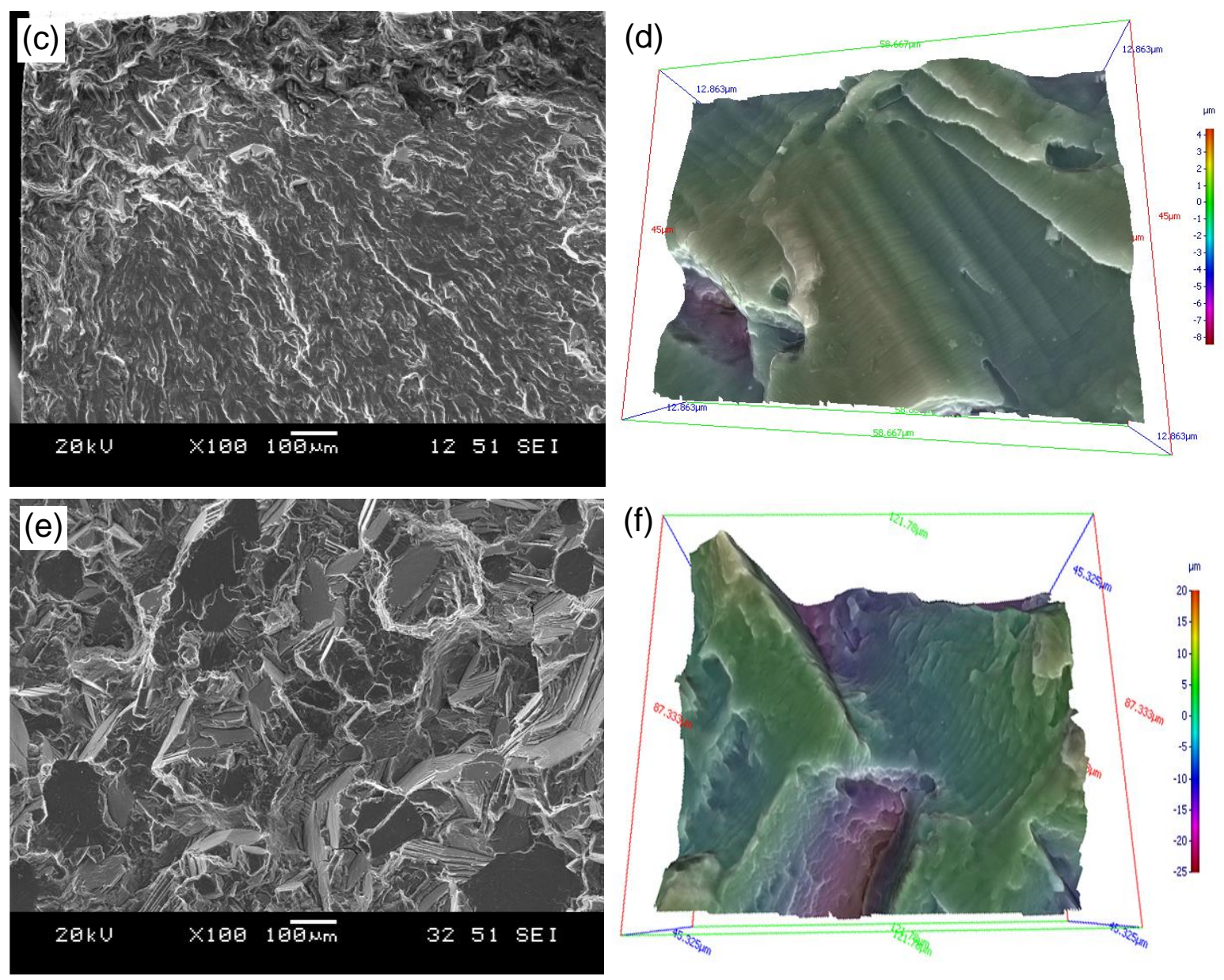

Figure 6.12: SEM micrographs of fracture surfaces near crack initiation area (left) and in the propagation zone (right) of the GW103K alloy in the (a) and (b) as-extruded, (c) and (d) T5, and (e) and (f) T6 states fatigued at a strain amplitude of $0.4 \%$.
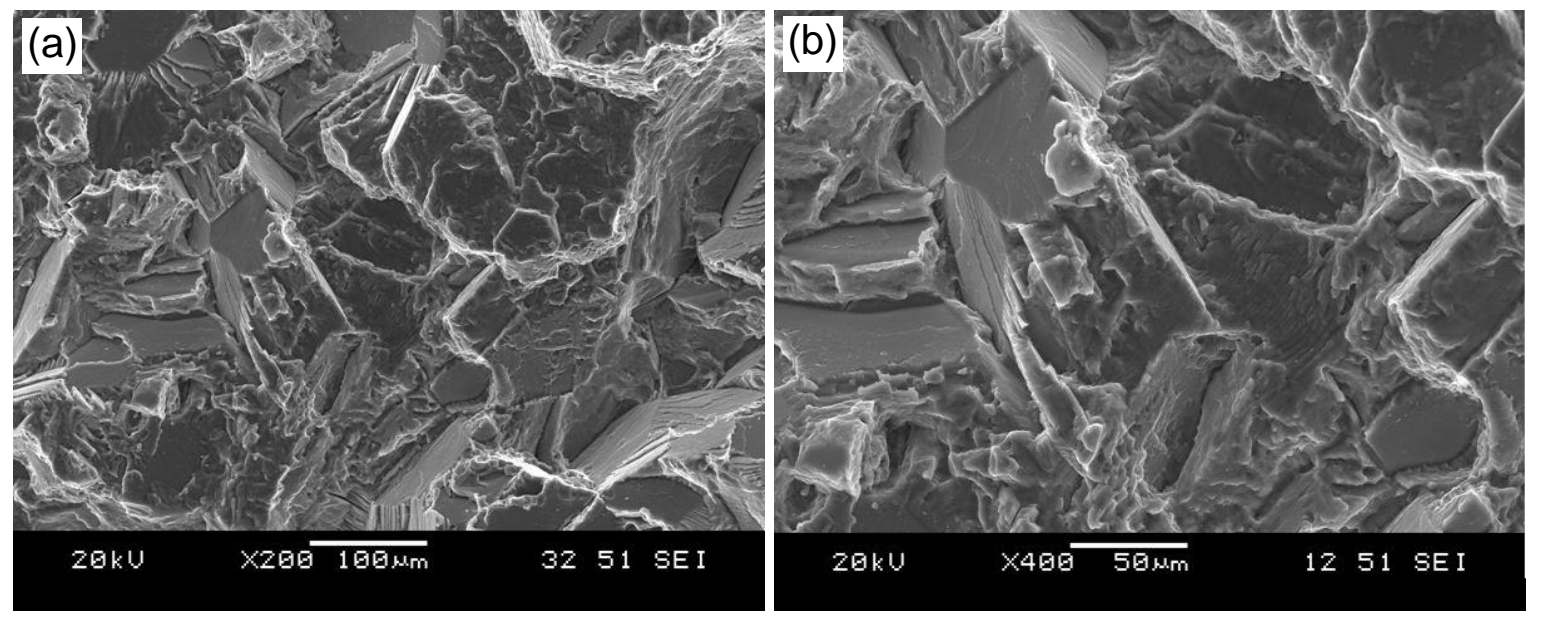

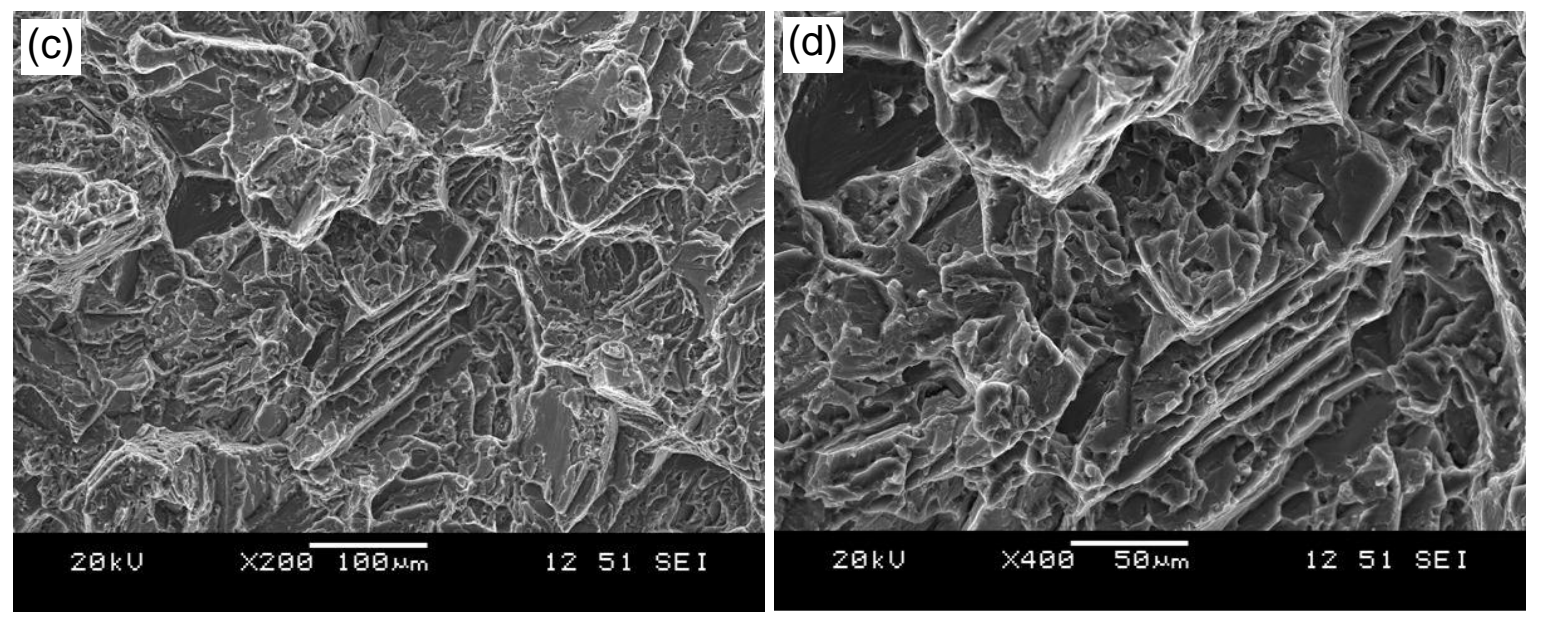

Figure 6.13: SEM micrographs of the fracture surface of the T6 sample fatigued at a strain amplitude of $0.4 \%$ in the region near the upper left corner ((a) and (b)), and region near the lower right corner $((c)$ and $(d))$ as indicated by the yellow dashed boxes in Fig. 6.11(c).

\subsection{Effect of RE elements}

\subsubsection{Effect on tensile properties}

The mechanical properties of alloys are known to be dependent on the alloying elements and the resulting microstructures including fine precipitates that appear after proper heat treatment. The process of adding other elements to a metal to increase its strength is commonly referred to as solid solution (or solute) strengthening. The effect of these solutes (or impurity atoms) is to obstruct the movement of dislocations. Edge dislocations for example can result in internal pressures in a crystal through the distortion of the periodic lattice. In the presence of impurity atoms with a larger atomic radius dilatational stresses are lowered and the energy of the system is decreased if the solute atoms are positioned below the edge dislocations. Therefore, extra stress is required to move dislocations away from the 
solute atom, which impedes their movement [225]. As seen from the periodic table, all RE elements including both series of lanthanides and actinides have an atomic radius larger than that of Mg. Also, the solid solubility of the RE elements in Mg is relatively high, e.g., Gd could dissolve up to 3.8 wt. $\%$ at about $200^{\circ} \mathrm{C}$ to replace the $\mathrm{Mg}$ atoms and form a substitutional solid solution [80]. Since the RE element ( $\mathrm{Gd}$ and $\mathrm{Y}$ ) atoms as well as $\mathrm{Zr}$ atoms in the GW103K alloy are all larger than $\mathrm{Mg}$ atoms, squeezing them into the $\mathrm{Mg}$ lattice would not only generate compressive stresses imposed on the nearby host atoms but also roughen the slip plane, thus make it more difficult for dislocations to move. It follows that the addition of $\mathrm{RE}$ elements to $\mathrm{Mg}$ even in the form of solid solution alone would improve the resistance to the motion of dislocations, and thereby increasing the yield strength. This would be the main reason why a fairly high strength of GW103K in the as-extruded state is observed (Table 6.1).

Regarding the precipitation strengthening, an alloy containing an adequate volume fraction of fine particles of an impurity phase through heat treatment will have a higher strength, due to the impediment of particles to the movement of dislocations. In the RE-Mg alloys fine and evenly oriented (the planes parallel to the prismatic or basal planes of $\mathrm{Mg}$ matrix) and uniformly distributed particles can improve the tensile properties [226]. On the other hand, Yin et al. [227] reported that relatively large size and small volume fraction of particles might have only small strengthening contributions. Thus, small sized particles or precipitates with an adequate volume fraction would have a significant contribution to the strengthening in RE-Mg alloys, as also reported in ref. [57]. As seen from Fig. 6.3(c), a large number of nano-sized dense and uniformly dispersed $\beta^{\prime}$ precipitates are obviously the primary 
strengthening phase of the GW103K alloy in the present T6 condition. It has also been reported that the $\beta^{\prime}$ precipitates, which form on the prismatic planes of $\alpha-\mathrm{Mg}$ matrix in a dense triangular arrangement $[57,104,119,228]$, are perpendicular to the basal plane of $\alpha-\mathrm{Mg}$ and very thermally stable at $250^{\circ} \mathrm{C}$ [227]. Such $\beta^{\prime}$ precipitates are particularly effective to impede the movement of dislocations on the basal planes [104,228], thus significantly increase the strength of the Mg alloy in the T6 state as seen from Fig. 6.5 and Table 6.1. Nie and Muddle [119] also reported that the enhanced tensile strength in the $\mathrm{Mg}-6 \mathrm{Gd}-1 \mathrm{Zn}-0.6 \mathrm{Zr}$ alloy resulted from a uniform and dense distribution of basal precipitate plates. As mentioned earlier, while the T5 heat treatment may not result in the same level of precipitates as in the T6 state due to the lack of solution treatment stage to form a supersaturated solid solution, the smaller grain size shown Fig. 6.1(c) would greatly enhance the strength according to the Hall-Petch relationship [34], in comparison with the much larger grain size in the T6 state (Fig. 6.1(e)). As the result, the tensile properties in the T5 state are nearly the same as those in the T6 (Fig. 6.5 and Table 6.1).

\subsubsection{Effect on cyclic deformation characteristics}

As compared to the as-extruded GW103K alloy, some obvious improvements in the cyclic deformation resistance have been observed in the T5 and T6 conditions. For example, the maximum and minimum peak stresses in the T5 and T6 conditions were much higher than those in the as-extruded condition in different hysteresis loops as seen from Fig. 6.6. At a given total strain amplitude the cyclic stress amplitude increased and plastic strain amplitude

decreased after the T5 and T6 heat treatment, with a big change from the as-extruded state to 
T5 state and a relatively small change from T5 to T6 state (Fig. 6.7 and Fig. 6.8). All of these changes during cyclic deformation corresponded well to those in the monotonic tensile properties (Fig. 6.5 and Table 6.1). To further identify the difference from the RE-free Mg alloys, the stress-strain hysteresis loop of the mid-life cycle for an AM30 extruded alloy is plotted in Fig. 6.14, along with that of the RE-containing GW103K alloy in various states. It is seen from Fig. 6.14 that the hysteresis loops of AM30 alloy showed a strong Bauschingerlike effect associated with a skewed asymmetrical shape.

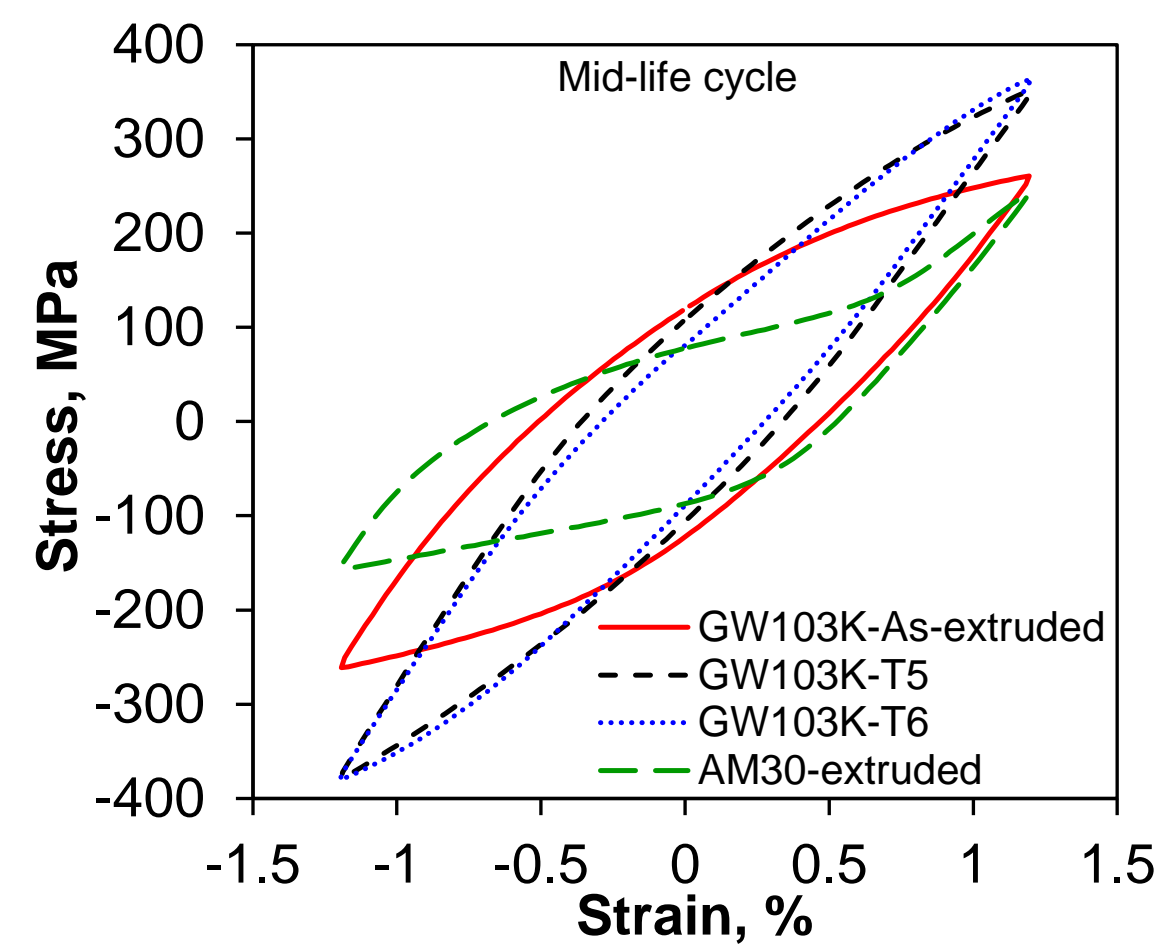

Figure 6.14: Typical stress-strain hysteresis loops of the mid-life cycle at a given total strain amplitude of $1.2 \%$ and strain ratio of $R_{\varepsilon}=-1$ for the extruded AM30 and GW103K alloy in different states, respectively.

While the maximum peak stress of extruded AM30 alloy was close to that of the GW103K alloy in the as-extruded state, the compressive yielding of the AM30 alloy in the descending phase occurred much earlier, giving rise to a much smaller minimum peak stress (absolute 
value). Similar results were also reported by other investigators $[26,28]$. The ratio of the compressive-to-tensile peak stress at a total strain amplitude of $1.2 \%$ was $\sim 0.55$ for AM30 alloy, and almost 1 for the GW103K alloy in different states, indicating that the tensioncompression asymmetry was no longer existent in the present GW103K, irrespective of alloy states. Typically, the tension-compression yield asymmetry in polycrystalline alloys is directly related to the presence of strong crystallographic texture which dominates the orientation of slip or twinning planes and directions relative to the externally applied stress [229]. Yin et al. [182] reported that randomly textured casting samples exhibited almost no yielding asymmetry. Likewise, Patel et al. [152,153,155] also observed no yielding asymmetry in the super-vacuum die cast or semi-solid processed AM60B and AZ91D Mg alloys, which was in agreement with the results of GW103K alloy. As mentioned earlier in section 6.2, the GW103K alloy showed relatively weak textures (Fig. 6.4) due to the presence of RE-rich particles and smaller grain sizes (which resulted from the Zener-pinning effect) as compared to the RE-free Mg alloys (Fig. 6.1 and Fig. 6.2). As seen from Fig. 6.15 which shows the distribution of deformation features in the area near the fracture surface in the fatigued samples of AM30 alloy and GW103K alloy in different states, it can clearly seen that the grain sizes of the RE-Mg alloy were smaller than the RE-free AM30 alloy even after the T6 heat treatment (Fig. 6.15(d) vs. (a)), which were partially responsible for the nearly symmetrical hysteresis loops (Fig. 6.6 and 6.14) via reduced twinning activities. It was reported that grain size had a significant effect on the tendency of twinning because the energy required to form twin interfaces was particularly high in the fine-grained $\mathrm{Mg}$ alloy [180], and the difference between the tensile and compressive yield strengths generally decreased with decreasing grain size [230]. More obvious and dense residual twins appeared 
near the fracture surface area of RE-free AM30 alloy as shown in Fig. 6.15(a), while twins could barely be visible in the near fracture surface area of the as-extruded GW103K alloy (Fig. 6.15(b)). Although a few twins could be seen to occur in the grains oriented favorably as the grain sizes increased considerably due to the T6 heat treatment (as indicated by arrows in Fig. 6.15(d)), the number of twins was limited, which was mostly replaced by the fine and uniformly distributed slip lines/bands in the RE-containing GW103K alloy in all the states (Fig. 6.15(b)-(d)).
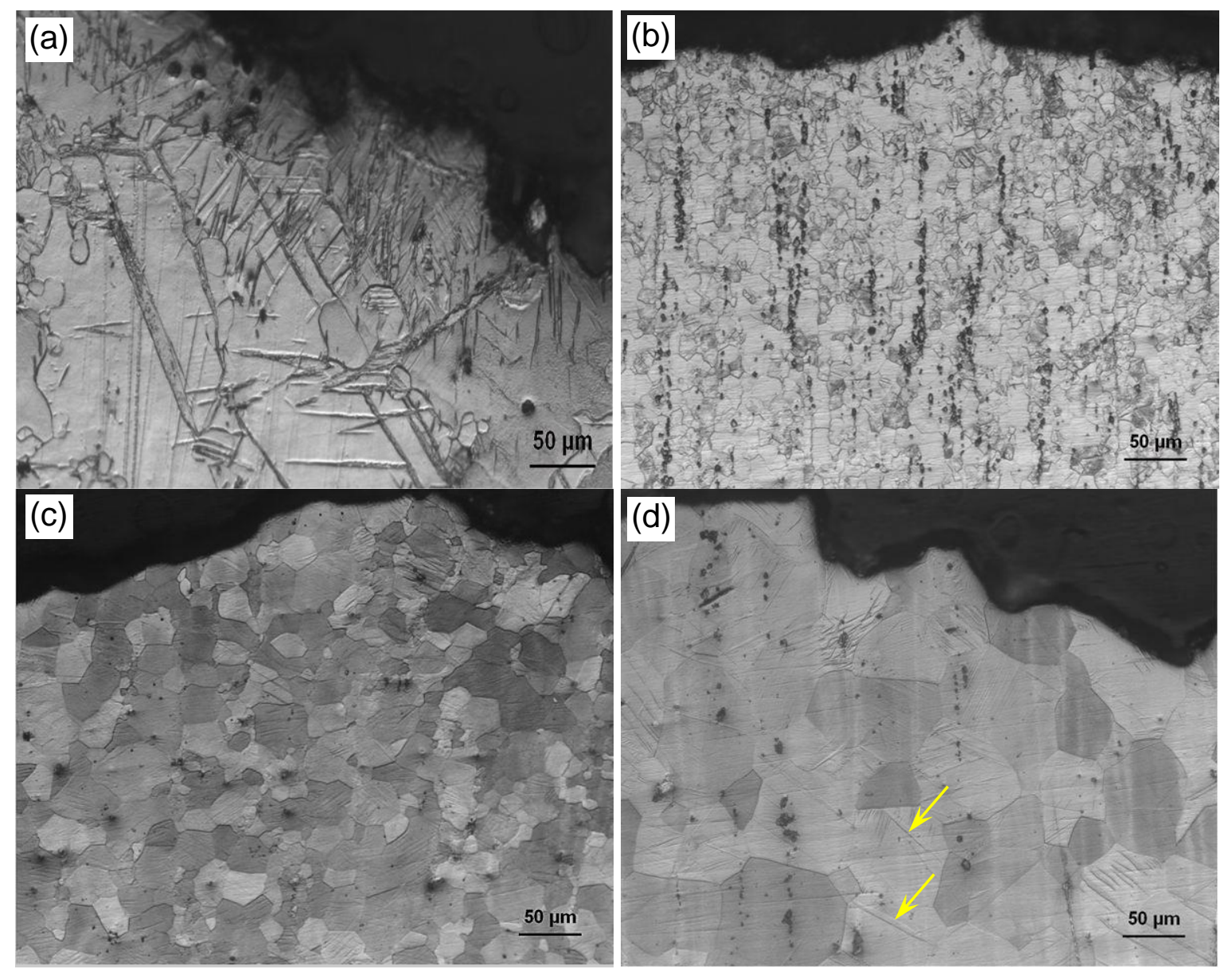

Figure 6.15: Optical micrographs in the area near the fracture surface at a strain amplitude of $1.2 \%$, showing the distribution of deformation features in the fatigued samples of (a) AM30 alloy, (b) as-extruded, (c) T5, and (d) T6 states of the GW103K alloy, respectively. 
Such an important change in the deformation mode from predominantly twinning in the REfree AM30 Mg alloy to mainly dislocation slip in the RE-containing GW103K alloy obviously resulted from (i) the grain refinement (Fig. 6.1), (ii) the uniformly-distributed large number density of bamboo leaf shaped $\beta^{\prime}$ precipitates (Fig. 6.3), and (iii) the texture weakening (Fig. 6.4) in the GW103K alloy, in comparison with AM30 Mg alloy. The combined role of these factors is responsible for the reduction or absence of the hysteresis loop asymmetry (Figs 6.6 and 6.14), the increase in the stress amplitude (Fig. 6.7) and the decrease in the plastic strain amplitude at a given total strain amplitude (Fig. 6.8), and eventually the increase in the fatigue life (Fig. 6.9) via suppressing the twinning activities in the GW103K alloy, as compared to the RE-free AM30 Mg alloy. The vital role of the REelements in this regard will be discussed further in the following section.

\subsubsection{Interaction between twins and precipitates}

An important factor that influences the formation of twins in the pull-push cyclic deformation in RE-Mg alloys is precipitates [183]. The presence of precipitates in the path of a migrating twin boundary can significantly alter the twinning behavior of RE-Mg alloys. As mentioned in earlier section 6.1.3, the presence of $\beta^{\prime}$ precipitates has been confirmed in RE$\mathrm{Mg}$ alloys as shown in Fig. 6.3. It was reported that this kind of precipitates can act as obstacles which bring about strengthening by pinning the twinning dislocations and restricting boundary motion. The interaction between twinning and precipitates has been considered to arise primarily from the difficulty experienced by the migrating twin boundaries during the propagation of the twin through densely distributed precipitates [229]. 
To better understand the interaction between a twin and plate-shaped $\beta^{\prime}$ precipitates, two schematic illustrations showing the barricade of a precipitate/particle to twinning are plotted in Figs 6.16 and 6.17 (two-dimensional and three-dimensional view as shown in Fig. 6.16 and Fig. 6.17, respectively). In Fig. 6.16, the coordinate system is established with the Y-axis being parallel to the $c$-axis [0001] of an hcp unit cell in the Mg matrix and the $\mathrm{X}$-axis being oriented in the direction of $[10 \overline{1} 0]$ which is positioned on the basal plane, whereas the threedimensional coordinate system is established in the hcp unit cell in the $\mathrm{Mg}$ matrix with three axes, i.e., $\mathrm{a}_{1}[2 \overline{1} \overline{1} 0], \mathrm{a}_{2}[\overline{1} 2 \overline{1} 0]$, and $\mathrm{a}_{3}[\overline{1} \overline{1} 20]$ which are positioned on the basal $\{0001\}$ plane as indicated by solid red arrows in Fig. 6.17 and all the three axes are oriented at an angle of $120^{\circ}$ to one another, with the $c$-axis [0001] being perpendicular to the basal $\{0001\}$ plane. As reported by He et al. [104] and Nie [228], the stacking type of atoms in $\beta^{\prime}$ precipitates observed in the RE-Mg alloys is the same as that of the hcp $\alpha-\mathrm{Mg}$ matrix. As mentioned earlier in section 6.1.3, the orientation of $\beta^{\prime}$ precipitates has been verified as $c$ base-centered orthorhombic system and it was observed to form on the prismatic planes of the $\mathrm{Mg}$ matrix in a dense triangular arrangement $[57,76,104,118,119,228]$. Thus, the precipitate could be plotted vertically to the $\mathrm{X}$-axis (the basal plane of the Mg matrix) in Fig. 6.16, while in Fig. 6.17 the precipitates could be plotted in the basal plane of the $\alpha$-Mg matrix in such a way that the orientation relationship between the $\beta^{\prime}$ precipitate and matrix patterns is $[001]_{\beta^{\prime}} / /[0001]_{\alpha}$, and $[100]_{\beta^{\prime}} / /[2 \overline{1} \overline{1} 0]_{\alpha}$ as reported in refs. $[57,76,104,119]$. In the $\mathrm{Mg}$ alloys, $\{10 \overline{1} 2\}$ extension twinning is normally activated when a compressive stress is applied perpendicular to the [0001] $c$-axis or parallel to the $\{0001\}$ basal plane $[16,231]$. Using the $\mathrm{Mg}$ lattice parameters of $a=0.32 \mathrm{~nm}$ and $c=0.52 \mathrm{~nm}$ [179], one can readily 
calculate the angle between the $\{0001\}$ basal plane and the $\{10 \overline{1} 2\}$ twinning plane $K_{1}$ or the twinning direction $\eta_{1}[\overline{1} 011]$ residing on the twinning plane $K_{1}$ to be $43.15^{\circ}$, as indicated in Fig. 6.16. Such a change is similar to re-orienting the basal planes of the matrix to those indicated by the fine parallel lines inside the twin, which are inclined at an angle of $86.3^{\circ}$ to the fine horizontal lines, as shown in Fig. 6.16. Due to the unique nature of twin characterized by the perfectly symmetrical (or mirror) image of atoms with respect to the twinning plane, the inclined fine parallel lines within the twin must have an atomic arrangement identical to that represented by the fine horizontal lines. Therefore, the inclined fine parallel lines inside the twin indeed stand for the $\{0001\}$ twin basal planes.

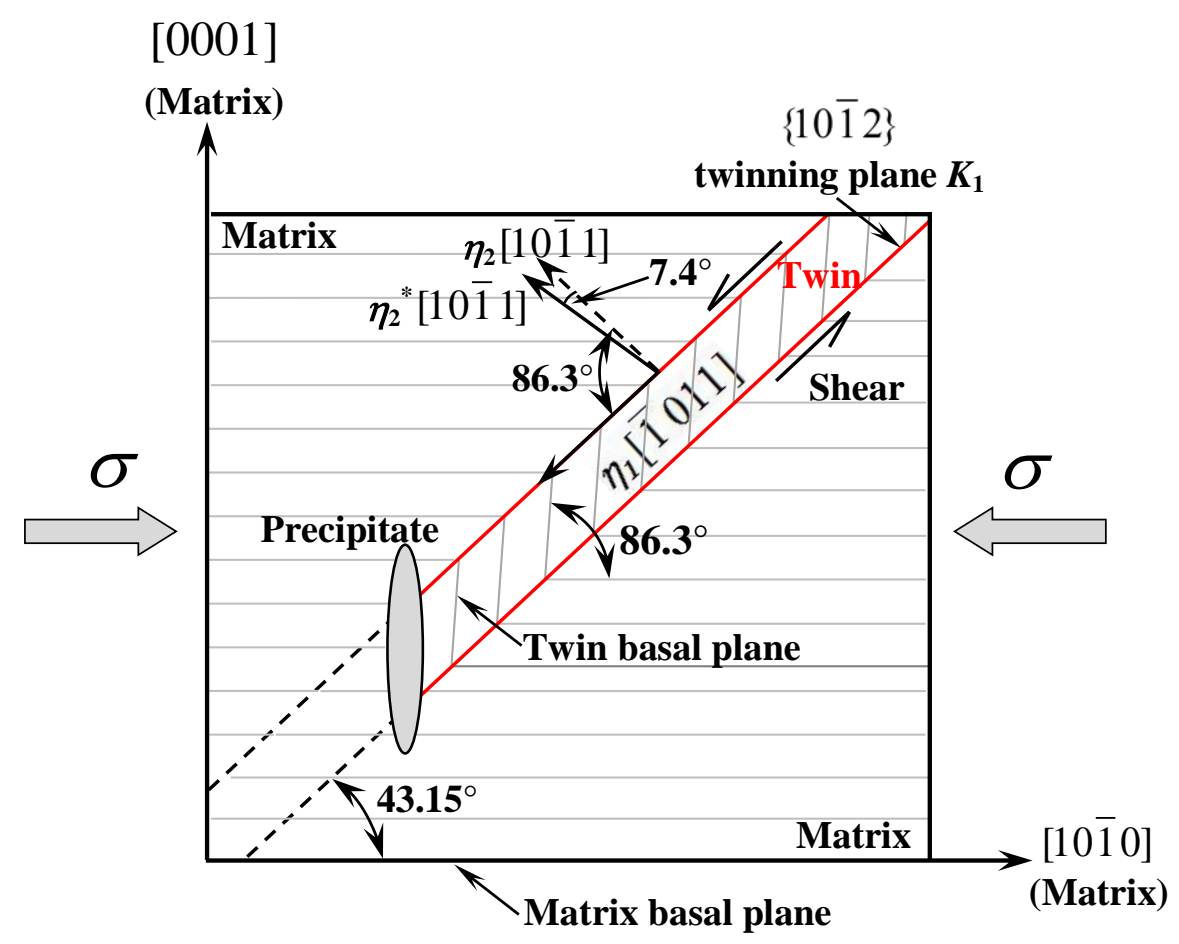

Figure 6.16: A schematic illustration of the interaction between a plate-shaped precipitate and $\{10 \overline{1} 2\}$ twin in the GW103K alloy. 
In Fig. 6.17, the (1 $\overline{1} 02)$ extension twinning plane is shown as a shaded color plate and the twinning direction [ $\overline{1} 101]$ is pointed using a dashed red arrow. If the particle/precipitate cannot be sheared during twinning, as the twinning ledge approaches the precipitate there exists a back stress arising from the harder and more rigid precipitate, which would hinder the free shear of the moving twin ledge and must be overcome by the increasing stress applied $[16,183]$.

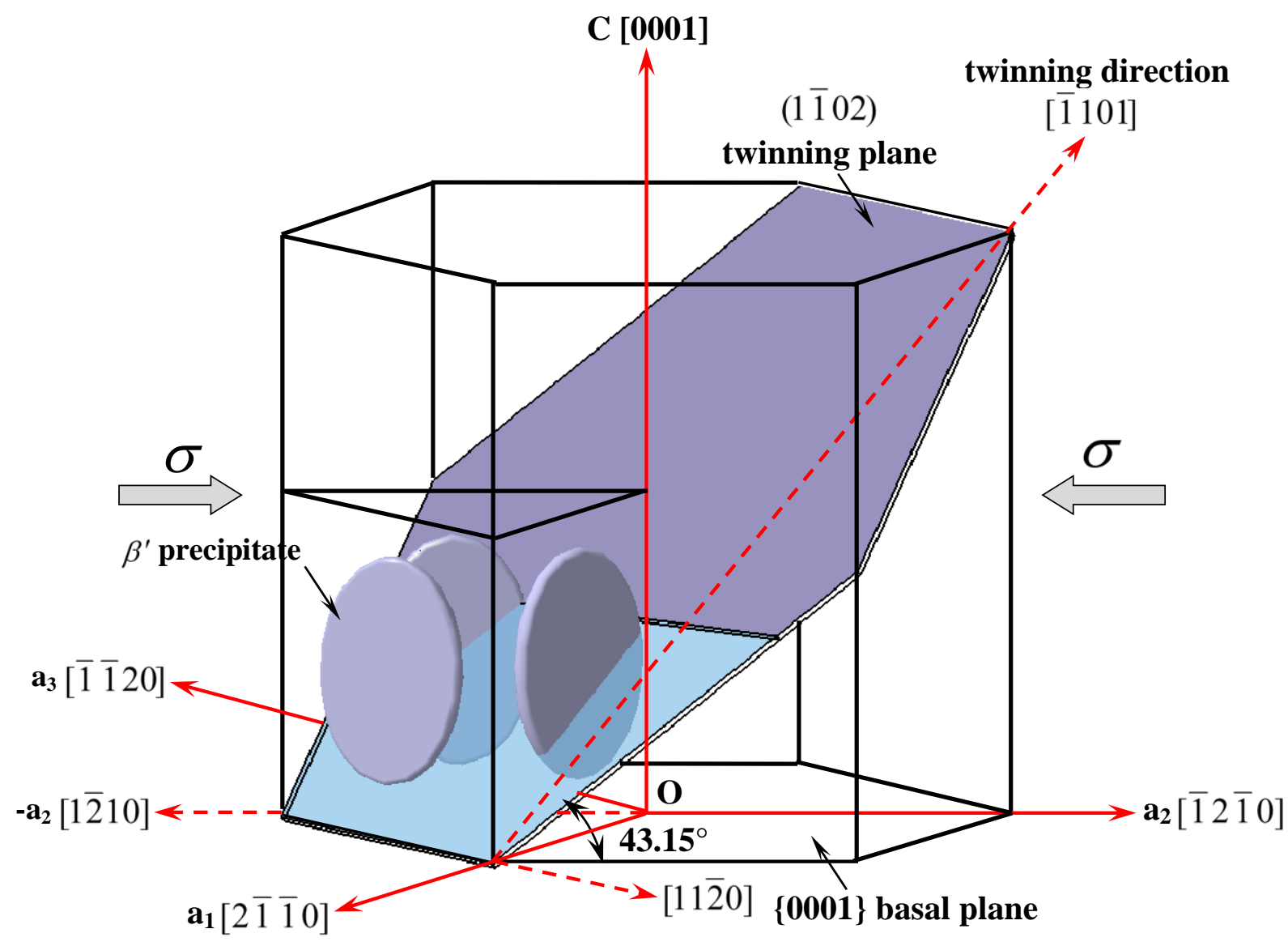

Figure 6.17: A schematic illustration of the interaction between $(1 \overline{1} 02)$ twin and $\beta$ ' precipitate with an orientation relationship of $[100]_{\beta^{\prime}} / /[2 \overline{1} \overline{1} 0]_{\alpha}$ and $[001]_{\beta^{\prime}} / /[0001]_{\alpha}$ in the GW103K alloy.

The portion of the matrix in the absence of twinning due to the blockage of the precipitate is indicated by the dashed lines in Fig. 6.16 and the un-twinned portion of the matrix in the absence 
of twinning due to the blockage of the triangular $\beta^{\prime}$ precipitates is indicated by the transparent cyan color in the twinning plane in Fig. 6.17. Furthermore, when the twinning shear reaches the interface between the matrix and the $\beta^{\prime}$ precipitate, the movement of atoms towards the precipitate should be accommodated by the basal slip of the matrix near the precipitate/matrix interface [183]. It should be noted that, the basal slip in the matrix would also be impeded by the $\beta^{\prime}$ precipitate, thereby providing an opportunity for the shear to be accommodated by basal slip inside the $(1 \overline{1} 02)$ twin instead of the parent matrix. Then the need for the additional basal slip on a local scale around the precipitate is likely to increase the stress required to form the twin [183]. It should be pointed out that the schematic diagram in Fig. 6.17, which is not drawn to scale since the precipitates were larger than the hcp unit cell of $\alpha$-Mg matrix, illustrates only a trio of $\beta^{\prime}$ precipitates in a given grain, so that partial twinning in the grain would be possible. As seen from the TEM image shown in Fig. 6.3 taken at a magnification of 30,000×, a large number of uniformly-dispersed triangular groups of plate-shaped $\beta^{\prime}$ precipitates are present in a given grain in the GW103K alloy. As a result, the resistance to the formation of twins in the GW103K alloy would be expected to be so large that almost no twinning would be possible and the dislocation slip became a predominant deformation mode as corroborated from the slip lines shown in Fig. 6.15.

\subsection{Summary}


The following conclusions can be drawn from the low cycle fatigue tests of GW103K alloy in the as-extruded, T5 and T6 conditions under varying strain amplitudes:

1. The grain size of the as-extruded GW103K alloy was fairly small (about $12 \mu \mathrm{m}$ ) in comparison with the RE-free extruded Mg alloys. The average grain size increased to about $25 \mu \mathrm{m}$ after $\mathrm{T} 5$ peak-aging at $225^{\circ} \mathrm{C}$ for $16 \mathrm{~h}$. More obvious grain growth (about $92 \mu \mathrm{m}$, being almost four times larger than that after T5 treatment) was observed in the T6 state mainly due to the solution treatment at $500^{\circ} \mathrm{C}$ for $2 \mathrm{~h}$ followed by the peak-aging at $225^{\circ} \mathrm{C}$ for $16 \mathrm{~h}$.

2. RE-containing particles were present in the GW103K alloy in all the three conditions. After T6 heat treatment, the RE-containing particles contained more $\mathrm{Zr}$ and had a more rounded shape. In particular, a high density of nano-sized and bamboo-leaf/plate-shaped $\beta^{\prime}$ $\left(\mathrm{Mg}_{7}(\mathrm{Gd}, \mathrm{Y})\right)$ precipitates were observed to distribute uniformly in the $\alpha-\mathrm{Mg}$ matrix.

3. Unlike the RE-free extruded Mg alloys, the present GW103K alloy in different conditions exhibited basically symmetrical hysteresis loops in tension and compression, suggesting the lack of the tensile-compressive yield asymmetry. This was predominantly attributed to (i) the grain refinement, (ii) the uniformly-distributed large number of bamboo leaf shaped $\beta^{\prime}$ precipitates, and (iii) the weaker texture.

4. The as-extruded GW103K alloy exhibited cyclic stabilization until failure up to a strain amplitude of $1.0 \%$. In the T5 and T6 states, cyclic stabilization occurred up to a strain amplitude of $0.6 \%$ followed by the slightly cyclic hardening from the strain amplitude of $0.8 \%$ to $1.2 \%$. Similar to the yield strength and ultimate tensile strength, the maximum and minimum peak stresses in the T5 and T6 conditions were much higher than that in the asextruded condition. 
5. The fatigue life of the GW103K alloy in different states was equivalent within the experimental scatter, which was longer than that of the RE-free extruded Mg alloys, and could be well described by the Coffin-Manson law and Basquin's equation.

6. SEM examinations revealed that fatigue cracks initiated from the specimen surface or nearsurface defects in all the three alloy states, and the initiation site contained some cleavage-like facets after T6 heat treatment. Crack propagation was characterized mainly by the typical fatigue striations. 


\section{CHAPTER 7}

\section{EFFECT OF STRAIN RATIO ON CYCLIC DEFORMATION OF EXTRUDED GW103K ALLOY}

While chapter 6 involves cyclic deformation behavior of GW103K alloy in different states with a fully reversed cycling (i.e., a strain ratio of $R_{\varepsilon}=-1$ ), there are no systematic studies reported on the low cyclic fatigue behavior of RE-containing Mg alloys with emphasis on the effect of strain ratio. The questions would be raised as to what are the effects of strain ratio on the cyclic deformation and low cycle fatigue resistance, and tension-compression asymmetry, how the cyclic stress response (i.e., cyclic hardening or softening) would be, and what is the effect of RE elements on the twinning-detwinning activities during cyclic deformation. Therefore, the aim of this chapter was to explore cyclic deformation behavior of an extruded GW103K alloy under a constant strain amplitude with different strain ratios.

\subsection{Hysteresis loops}

Fig. 7.1 shows typical stress-strain hysteresis loops of the first, second, and mid-life cycles at different strain ratios, $R_{\varepsilon}$, at a given strain amplitude of $0.8 \%$ of the extruded GW103K alloy. It is seen from Fig. 7.1(a) that at all strain ratios, the initial tensile phase of the first cycle was synchronized and followed essentially the same path, which suggested the occurrence of a nearly ideal Masing behavior, representing a special cyclic stress-strain response in some materials as reported by Chirst and Mughrabi [232]. With increasing strain ratio from - $-\infty$ (negative infinity) to 0.5 , the maximum/peak tensile stress $\sigma_{\max }$ increased from nearly zero or a few MPa to about 
$300 \mathrm{MPa}$, and the plastic deformation in the tensile phase became much more remarkable (Fig. 7.1(a)). In particular, there was a large amount of plastic deformation (Fig. 7.1(a)) in the tensile phase at zero or positive strain ratio (i.e., $R_{\varepsilon}=0.5$ and 0 ). This was related to the high positive mean strain value (i.e., the fixed values of maximum strain $\varepsilon_{\max }$ and minimum strain $\varepsilon_{\min }$ as given in Table 3.2). The shift of the hysteresis loops at $R_{\varepsilon}=0.5$ and $R_{\varepsilon}=0$ in Fig. 7.1(b) and (c) was also due to the control of strain limits. With decreasing strain ratio from 0.5 to $-\infty$, the minimum/peak compressive stress $\sigma_{\min }$ decreased from about $-170 \mathrm{MPa}$ to about $-270 \mathrm{MPa}$, where the $R_{\varepsilon}=0.5$ and $R_{\varepsilon}=0$ tests did not reach the compressive yielding of the GW103K Mg alloy. While the $R_{\varepsilon}=-1$ test gave essentially symmetrical hysteresis loops (Fig. 7.1(b) and (c)), the tests at other strain ratios apparently resulted in asymmetrical hysteresis loops with respect to the zero stress (Fig. 7.1(b)). However, the initially asymmetrical hysteresis loops became relatively more symmetric in tension and compression at the mid-life, as see in Fig. 7.1(c).

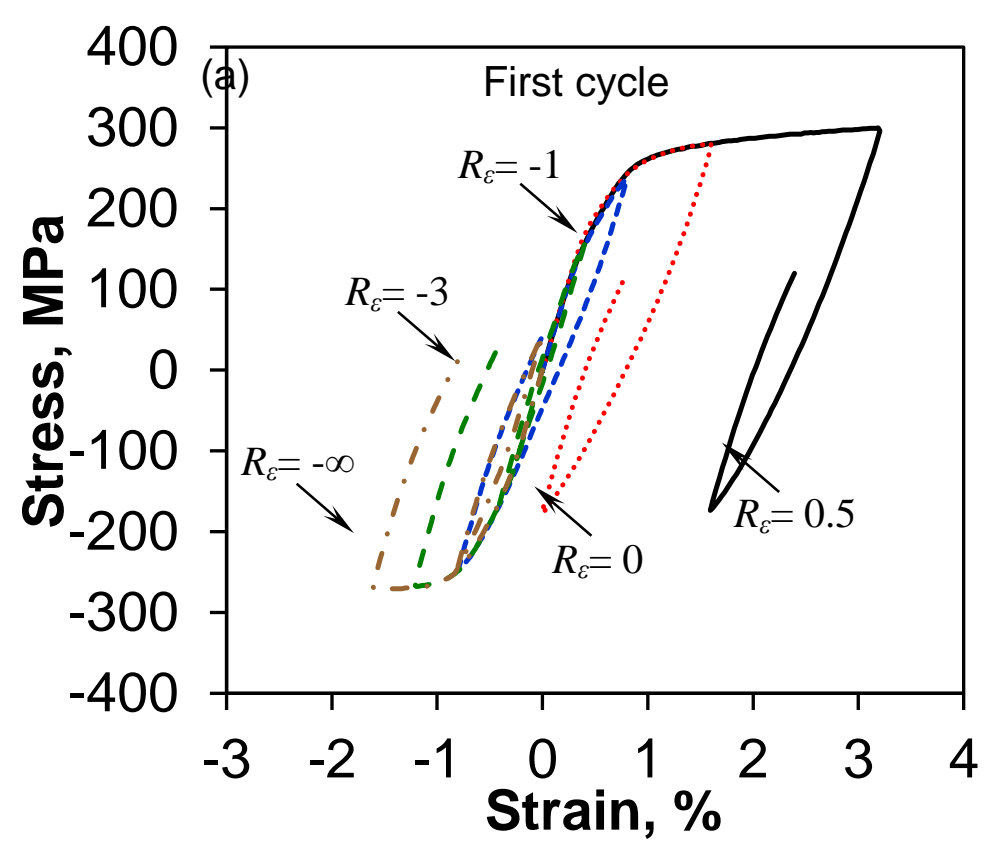



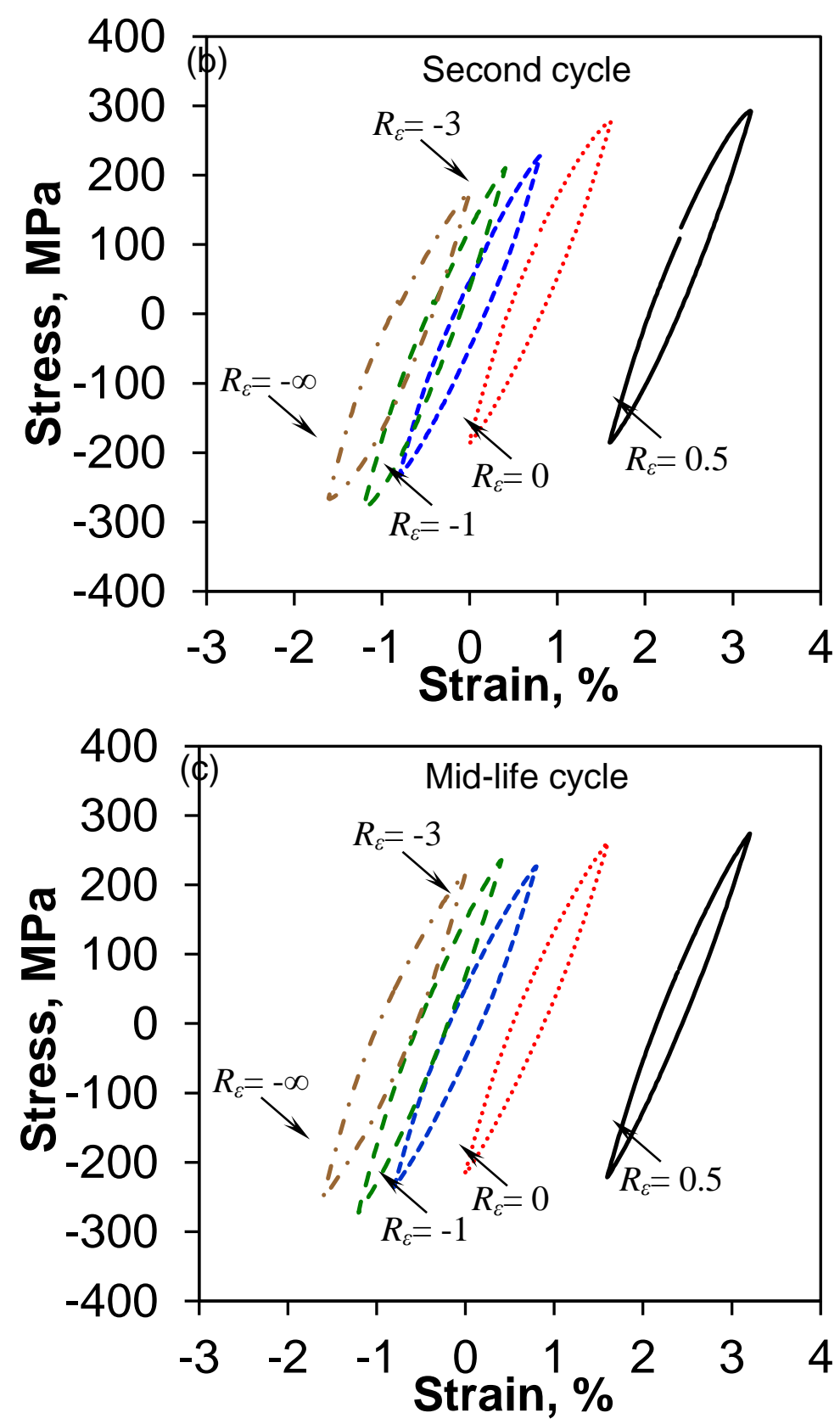

Figure 7.1: Typical stress-strain hysteresis loops of different cycles at different strain ratios at a given total strain amplitude of $0.8 \%$ of the extruded GW103K alloy, (a) First cycle, (b) Second cycle, and (c) Mid-life cycle. 


\subsection{Cyclic deformation response}

Fig. 7.2 shows the evolution of stress amplitudes with respect to the number of cycles at different applied strain ratios on a semi-log scale. It is seen that the GW103K alloy remained almost cyclically stable until failure at different strain ratios. However, the alloy showed initial abrupt increase in the stress amplitude from the first cycle to the second cycle at the strain ratio of $R_{\varepsilon}=-3$ and $-\infty$, then it remained almost constant until failure as with other strain ratios. It is noted that the rate of initial sudden change was higher at a strain ratio of $R_{\varepsilon}=-\infty$ than at a strain ratio of $R_{\varepsilon}=-$ 3 , since the compressive stress at $R_{\varepsilon}=-\infty$ was higher than that at $R_{\varepsilon}=-3$. The stress amplitude basically increased with increasing $R_{\varepsilon}$ value under the same strain amplitude of $0.8 \%$ and strain rate, except the case of $R_{\varepsilon}=-3$. This was attributed to the higher mean strain, which was in agreement with the results reported in refs. $[25,126,152]$ where the absolute value of high mean strain corresponded to the high stress amplitude. Unlike the RE-free extruded AM30 [28,35] and AZ31 [25-27] where cyclic stabilization occurred only at lower strain amplitudes of about $0.1 \%$ and $0.2 \%$, the GW103K alloy exhibited cyclic stabilization until failure at higher strain amplitude of $0.8 \%$. The variation of the plastic strain amplitude $\left(\Delta \varepsilon_{\mathrm{p}} / 2\right)$ during cyclic deformation is shown in Fig. 7.3 at different strain ratios. It is seen that at all strain ratios there existed a sudden drop of the plastic strain amplitude from the first cycle to the second cycle, except the fully reversed strain control (i.e., $R_{\varepsilon}=-1$ tests), then it remained almost constant until failure. Indeed, such an abrupt drop was associated with the existence of mean strain, and it increased with increasing absolute value of mean strain (Table 3.2) or with increasing deviation from zero mean strain $\left(\varepsilon_{\text {mean }}=0\right.$ or $\left.R_{\varepsilon}=-1\right)$. Similar sudden drops have been observed in an extruded AZ31 alloy [25]. It is further seen from Fig. 7.3 that as the $R_{\varepsilon}$ increased the plastic 
strain amplitude decreased. The cyclic stabilization exhibited in the present alloy at a fairly high strain amplitude of $0.8 \%$ indicated the beneficial effect of RE element additions.

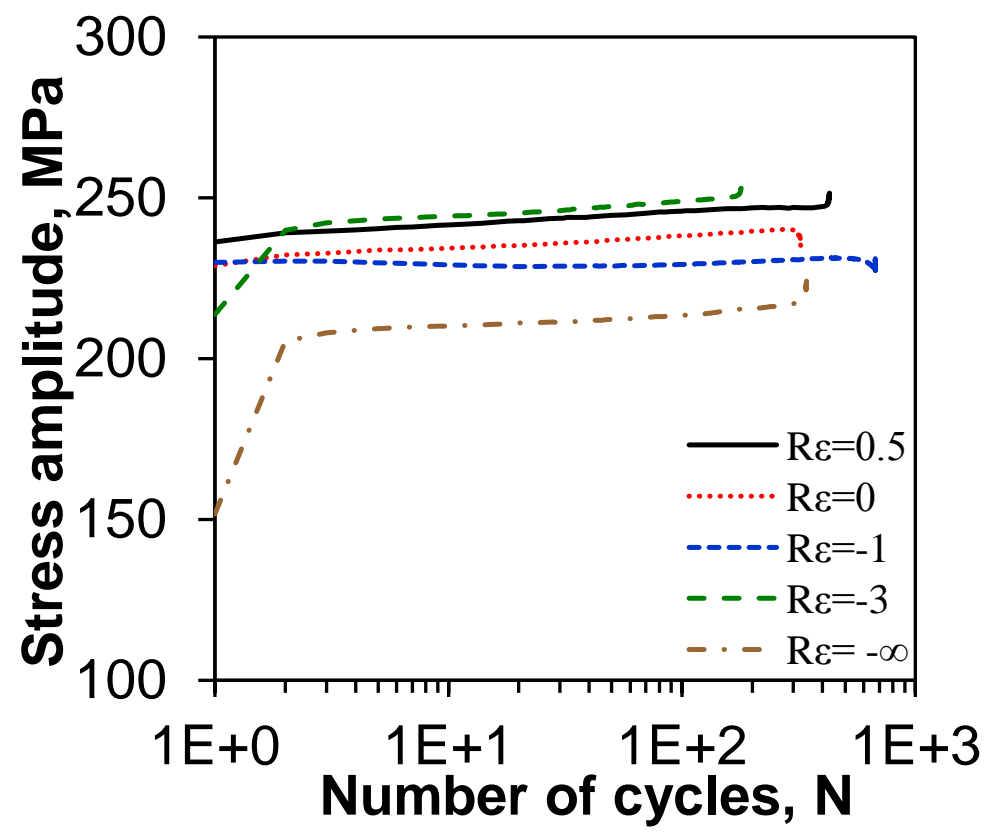

Figure 7.2: Stress amplitude vs. the number of cycles at different strain ratios at a given total strain amplitudes of $0.8 \%$ of the extruded GW103K alloy.

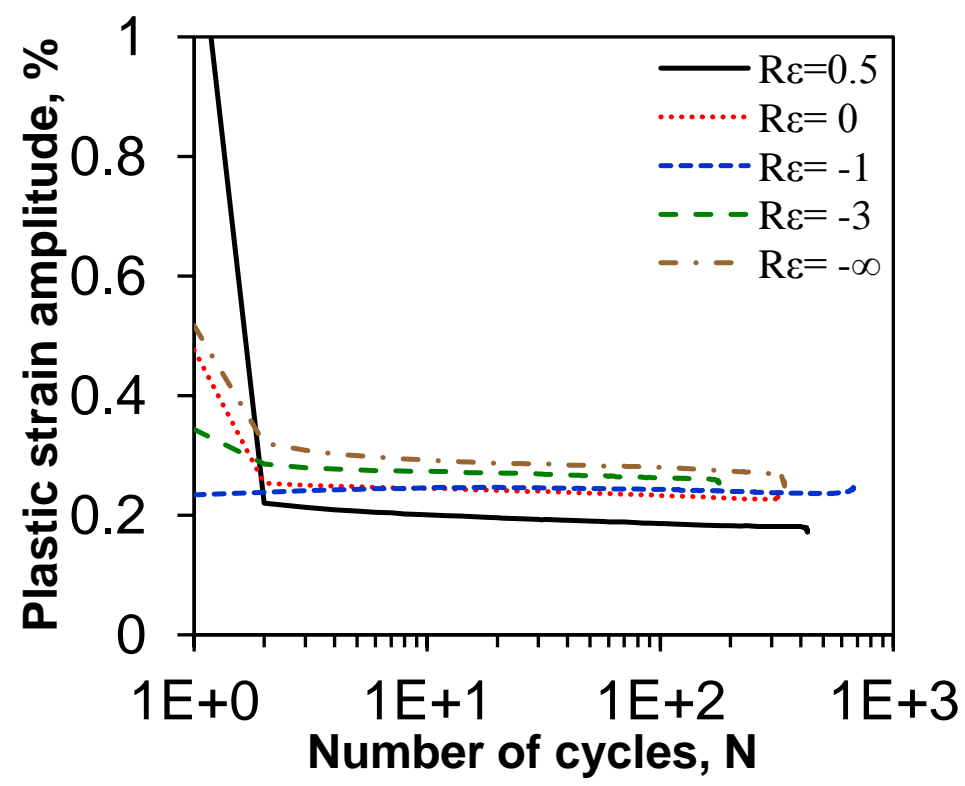

Figure 7.3: Plastic strain amplitude vs. the number of cycles at different strain ratios at a given total strain amplitudes of $0.8 \%$ of the extruded $G W 103 \mathrm{~K}$ alloy. 


\subsection{Fatigue life and fatigue parameters}

The fatigue life (i.e., the number of cycles to failure, $N_{f}$ ) as a function of strain ratio of the extruded GW103K alloy is plotted in Fig. 7.4. It is seen that the fatigue life increased with increasing strain ratio from $R_{\varepsilon}=-\infty$ to $R_{\varepsilon}=-1$, while it decreased from $R_{\varepsilon}=-1$ to $R_{\varepsilon}=0.5$, which seemed to be in agreement with the change of the plastic strain amplitude with the strain ratio (Fig. 7.3), as discussed above. It should be noted that the relatively low fatigue lives shown in Fig. 7.4 was due to the applied higher total strain amplitude of $0.8 \%$, giving rise to a higher cyclic stress amplitude (Fig. 7.2) and plastic strain amplitude. A lower total strain amplitude of $0.4 \%$ or $0.6 \%$ was used in $[25,152,155]$. The mean stresses at different strain ratios plotted as a function of a normalized cycle ratio $\left(N / N_{\mathrm{f}}\right)$ is shown in Fig. 7.5, where $N_{\mathrm{f}}$ is the fatigue life (the number of cycles to failure).

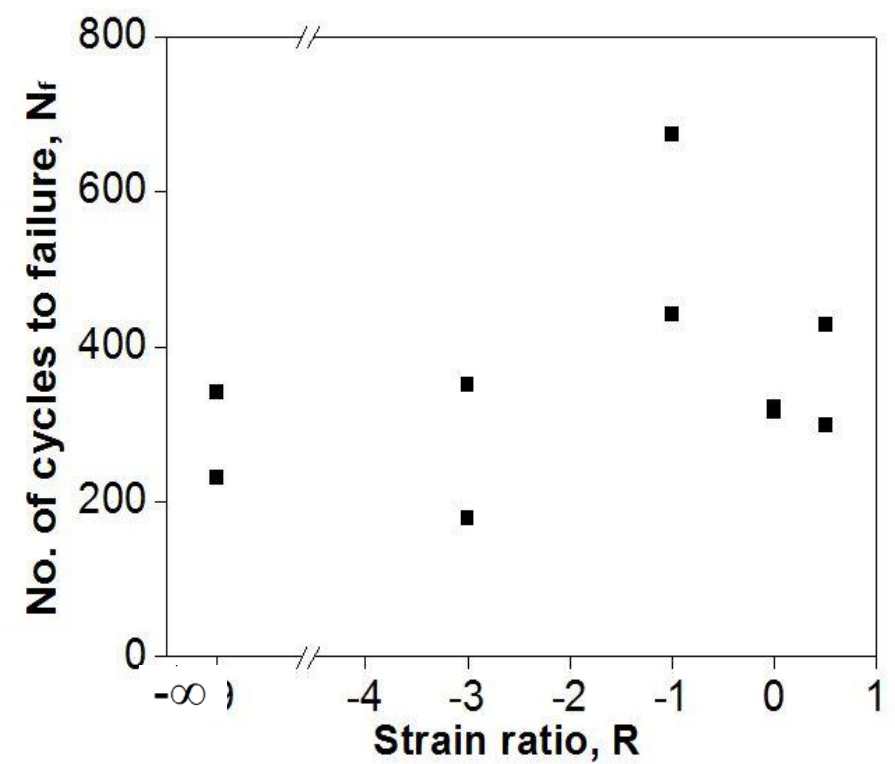

Figure 7.4: Number of cycles to failure vs. strain ratio for the extruded GW103K alloy tested at a given total strain amplitude of $0.8 \%$ and strain rate of $1 \times 10^{-2} \mathrm{~s}^{-1}$. 


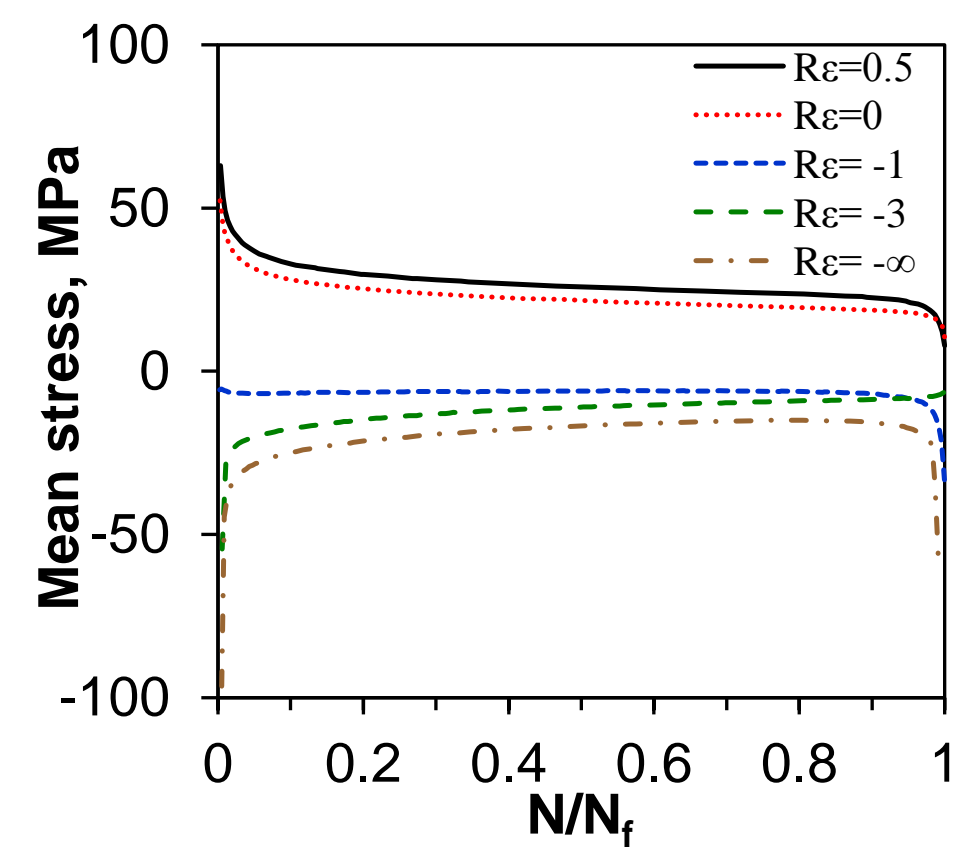

Figure 7.5: Mean stress vs. a normalized cycle ratio $\left(N / N_{f}\right)$ at different strain ratios in the GW103K alloy tested at a total strain amplitudes of $0.8 \%$.

It is seen that the initial mean stress decreased for $R_{\varepsilon}>-1$ and increased for $R_{\varepsilon}<-1$, and the stabilization basically occurred after approximately $10-20 \%$ of fatigue life for the applied strain ratios, except for the strain ratio of 0.5 and 0 where the mean stress decreased continuously. The mean stress at $R_{\varepsilon}=-1$ remained nearly zero or absent. Furthermore, the variation of the hysteresis loops with the strain ratio could also be expressed as a minimum-to-maximum stress ratio, $\sigma$ $\min / \sigma_{\max }$, versus the strain ratio (Fig. 7.6). The stress ratio in the first cycle increased drastically with increasing strain ratio, while the extent of change in the stress ratio in the mid-life cycle became much smaller. As expected, the stress ratio between the first cycle and the mid-life cycle was almost the same at $R_{\varepsilon}=-1$. However, the stress ratio in the mid-life cycle became lower when the strain ratio was smaller than -1 , and higher when the strain ratio was larger than -1 . Similar changes in the stress ratio have been reported in ref. [25]. 


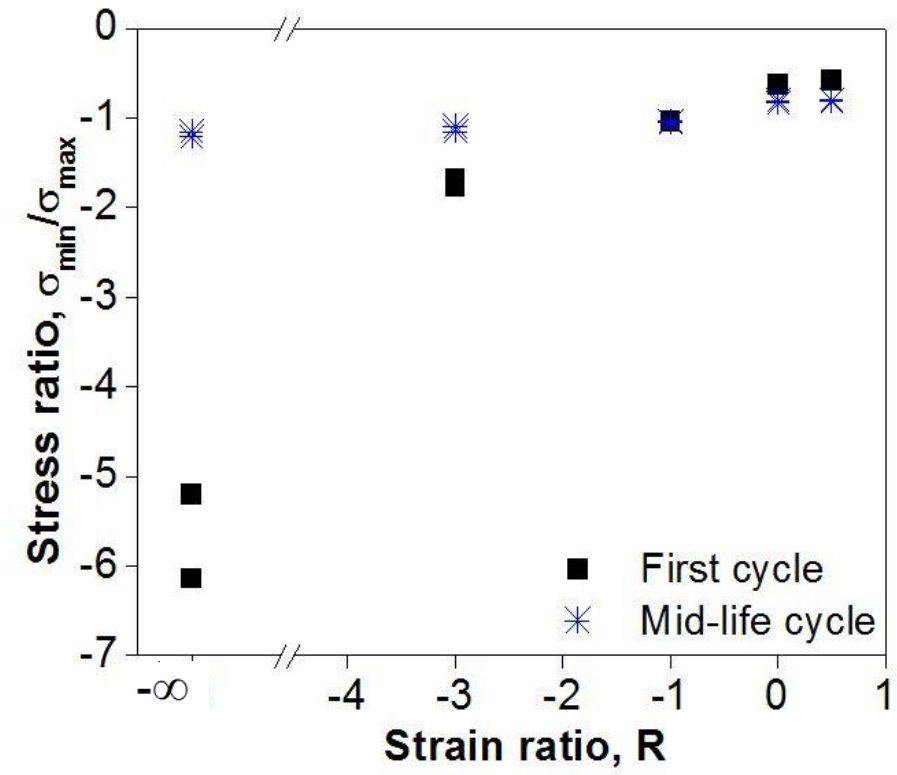

Figure 7.6: Stress ratio of the first cycle and the mid-life cycle as a function of the applied strain ratio.

\subsection{Fractography}

Fig. 7.7 shows an overall view of the fracture surfaces at a lower magnification for the specimens tested at a total strain amplitude of $0.8 \%$ and at a strain ratio of (a) 0.5 and (b) $-\infty$, respectively. It is seen that fatigue crack initiated basically from the specimen surface. The fracture surface near the crack initiation site at a larger magnification is shown in Fig. 7.8(a) and (b). The fracture surfaces at both strain ratios had similar features containing cleavage-like facets. Fatigue crack propagation was basically characterized by fatigue striations observed at higher magnifications (Fig. 7.9(a) and (b)), in conjunction with some secondary cracks. In addition, the fatigue striations were perpendicular to the crack propagation direction. 

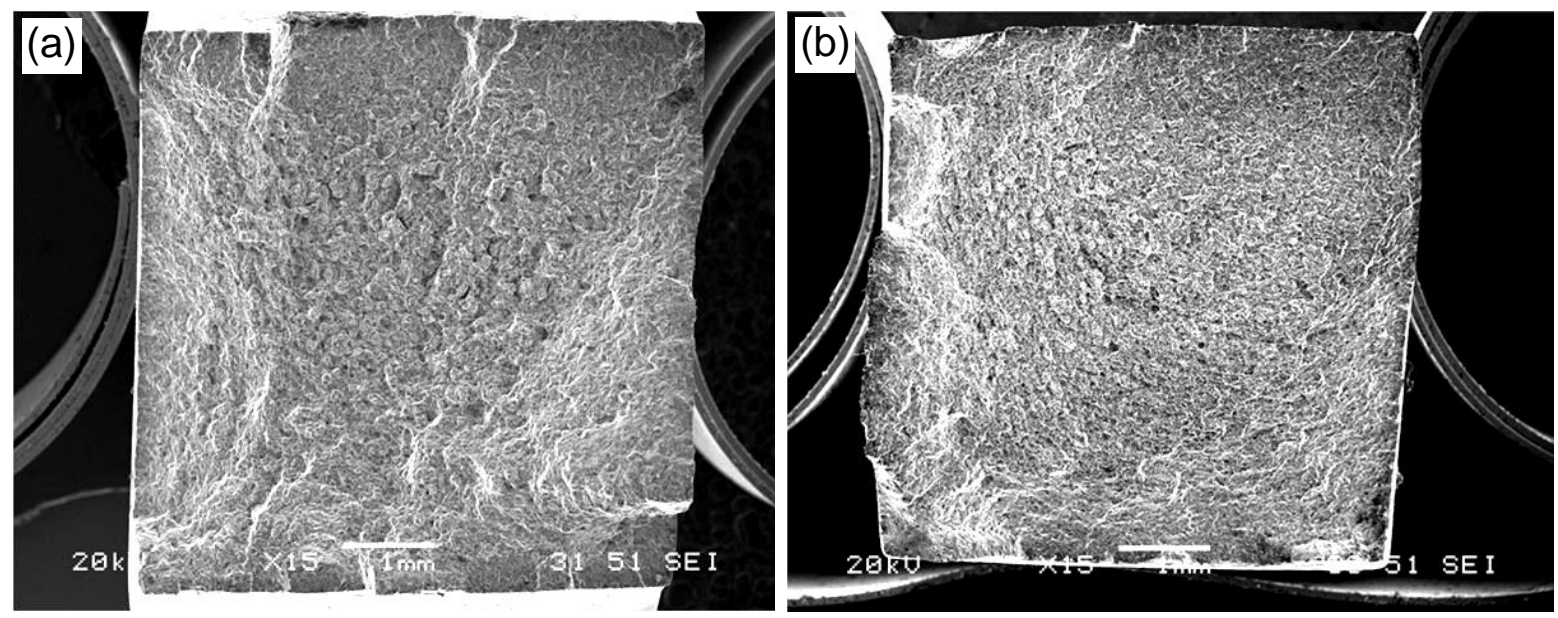

Figure 7.7: Typical SEM images of overall fracture surfaces of the extruded GW103K specimens fatigued at a total strain amplitude of $0.8 \%$ and at a strain ratio of (a) 0.5 and (b) $-\infty$.
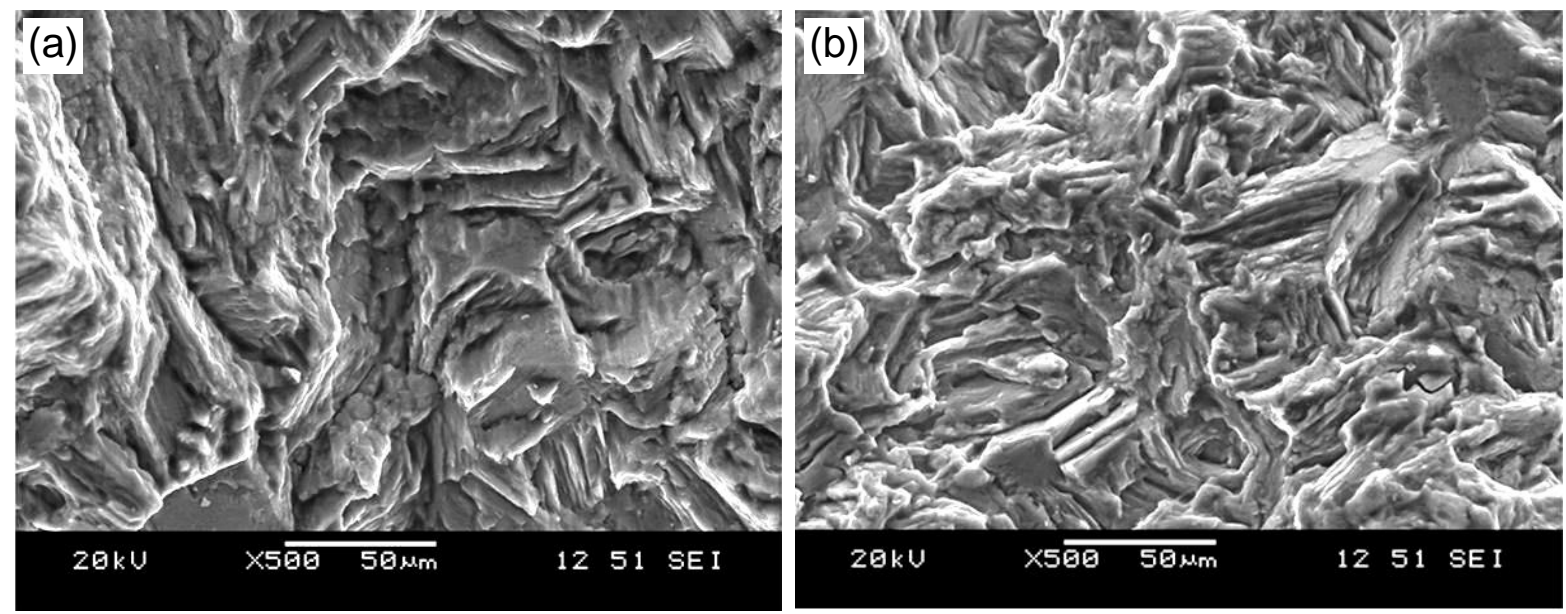

Figure 7.8: SEM micrographs of the fracture surface near crack initiation of the extruded GW103K specimens fatigued at a total strain amplitude of $0.8 \%$ and at a strain ratio of (a) 0.5 and $(b)-\infty$. 

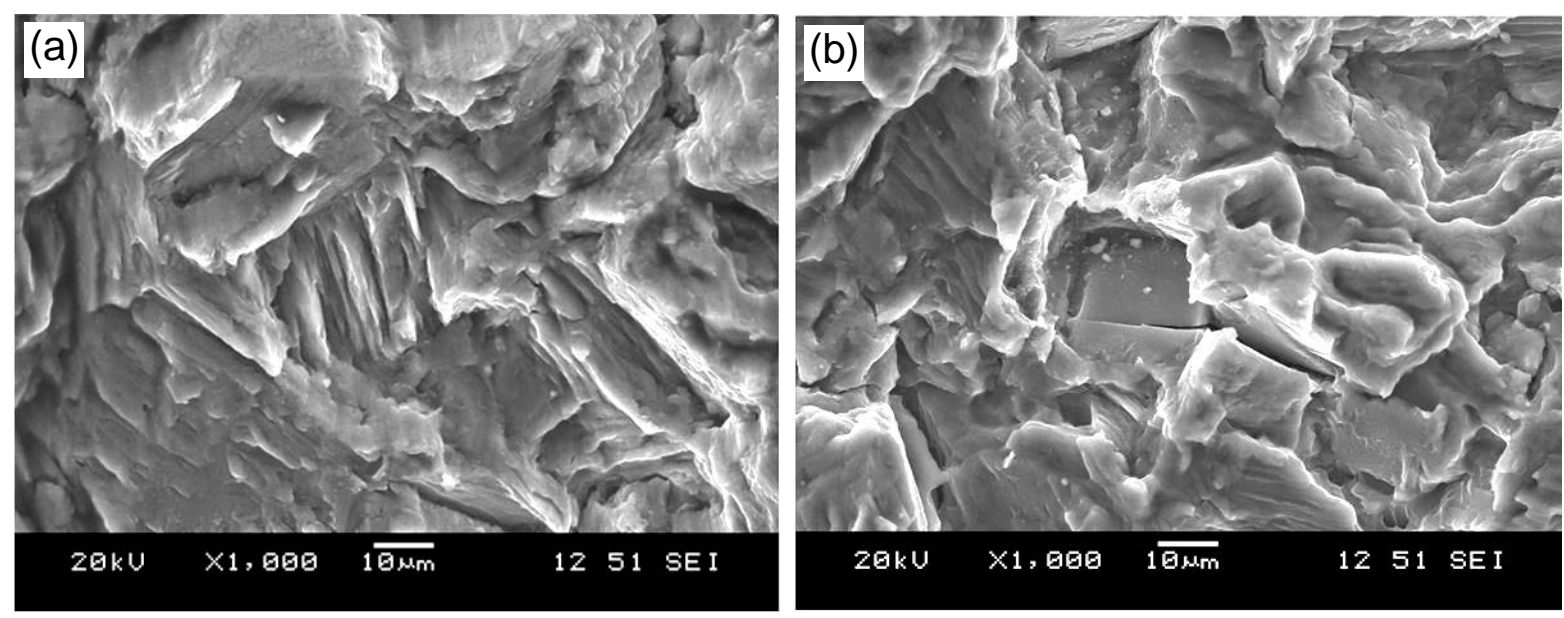

Figure 7.9: SEM micrographs of fatigue crack propagation region of the extruded GWIO3K specimens fatigued at a total strain amplitude of $0.8 \%$ and at a strain ratio of $(a) 0.5$ and $(b)-\infty$.

\subsection{Effect of strain ratio}

As mentioned above in section 7.2 , the strain ratio has a significant effect on the cyclic deformation characteristics of the GW103K alloy. For instance, due to the high positive mean strain values, a large amount of plastic deformation occurred in the tensile phase which can be observed from the hysteresis loops (Fig. 7.1(a)). In addition, with decreasing strain ratio, the hysteresis loops became wider and appeared more symmetric with respect to the zero stress (Fig. 7.1(b) and (c)), which was due to the occurrence of twinning-detwinning as reported in ref. [25]. The stress amplitude was also affected due to the high mean strain (Fig. 7.2) and it increased with increasing $R_{\varepsilon}$ values. The mean stress at $R_{\varepsilon}=-1$ was nearly absent, and the absolute value of the mean stress became larger when the strain ratio deviated more from $R_{\varepsilon}=-1$ (Fig. 7.5). As the cyclic deformation progressed, the mean stresses at different strain ratios shifted towards a reduced absolute value or exhibited a tendency of approaching zero mean stress (Fig. 7.5). This phenomenon is often referred to as the mean stress relaxation. It is clear that the mean stress 
relaxation occurred more drastically at the onset of cyclic deformation within the initial cycles of the fatigue life, beyond which (especially beyond the mid-life) it became increasingly stabilized. Also, the higher the initial mean stress (absolute value) was, the more significant the amount of mean stress relaxation. The non-stability in the mean stress (mean stress relaxation) at non-fully reversed strain control (i.e., $R_{\varepsilon} \neq-1$ tests) might be related to the initial big over-stretching (Fig. 7.1(a)). The nearly stable and positive/tensile mean stress over the majority of fatigue life decreased from about $+30 \mathrm{MPa}$ to about $-25 \mathrm{MPa}$ with decreasing strain ratio from $R_{\varepsilon}=0.5$ to $R_{\varepsilon}=-\infty$. This was in agreement with the results reported by Goodenberger and Stephens [154] on AZ91E-T6 cast magnesium alloy. It should be noted that the change in the stress ratio from the first cycle to the mid-life cycle in Fig. 7.6 indeed also indicated the mean stress relaxation or cycle-dependent relaxation.

\subsection{Comparison between GW103K alloy and RE-free extruded AM30 alloy}

Fig. 7.10 shows typical stress-strain hysteresis loops of the first and mid-life cycles at a total strain amplitude of $0.8 \%$ and at different strain ratios $\left(R_{\varepsilon}=0.5,-1\right.$, and $\left.-\infty\right)$ for the extruded GW103K alloy (solid lines) and AM30 alloy (dashed lines), respectively. The GW103K alloy exhibited hysteresis loops that were very different from those of the extruded RE-free AM30 alloy. Unlike AM30 alloy, the current RE-containing GW103K alloy exhibited nearly symmetrical hysteresis loops in shape with a higher stress amplitude (Fig. 7.10), which were somewhat similar to those of face-centered cubic (fcc) metals (e.g., $\mathrm{Al}, \mathrm{Cu}, \mathrm{Ni}$ ) as a result of the dislocation slip-dominated deformation in most materials [33]. This was due to the presence of 
RE-rich particles and finer grain sizes as presented in section 6.4.1. Moreover, the skewness of the hysteresis loops present in the extruded AM30 alloy decreased with increasing strain ratio, which was absent in the current GW103K alloy. Generally, the initial deformation asymmetry behavior of metals was associated with the Bauschinger effect [34]. From Fig. 7.10(b) it is clearly seen that the hysteresis loops of AM30 alloy showed a strong Bauschinger-like effect, which concurred with the results reported by other investigators $[26,28,34]$. The ratio of the compressive-to-tensile yield stress was $\sim 0.55$ for AM30 alloy, whereas it was almost 1 for GW103K alloy presented in section 6.5.2, indicating that there was nearly no difference in the tensile and compressive yielding (Fig. 7.10(b)). The difference between GW103K alloy and AM30 alloy could also be observed from the near fracture surface area in Fig. 7.11, where the distribution of residual twins in the fatigued samples of GW103K alloy (Fig. 7.11(a) $R_{\varepsilon}=0.5$, (c) $R_{\varepsilon}=-1$, and (e) $R_{\varepsilon}=-\infty$ ) and AM30 alloy (Fig. 7.11(b) $R_{\varepsilon}=0.5$, (d) $R_{\varepsilon}=-1$, and (f) $R_{\varepsilon}=-\infty$ ) is shown, respectively. With decreasing strain ratio from $R_{\varepsilon}=0.5$ to $R_{\varepsilon}=-\infty$, the amount of twin formation increased (Fig. 7.11) in both alloys.

However, compared to the GW103K alloy (Fig. 7.11(a), (c), and (e)), denser and more obvious residual twins were present near the fracture surface of AM30 alloy (Fig. 7.11(b), (d), and (f)). The formation of these twins was attributed to an insufficient number of slip systems in magnesium alloys deformed at room temperature arising from the HCP crystal structure with low crystal symmetry $[16,108]$. The absence of dense twins in the GW103K alloy suggested that twinning would not be a predominant deformation mode in the cyclic deformation of this alloy, and the basal slip of dislocations would become a predominant deformation mechanism as 
corroborated by the nearly symmetrical hysteresis loops (Fig. 7.1 (c) and Fig. 7.10(b)) like the common fcc metals [33].
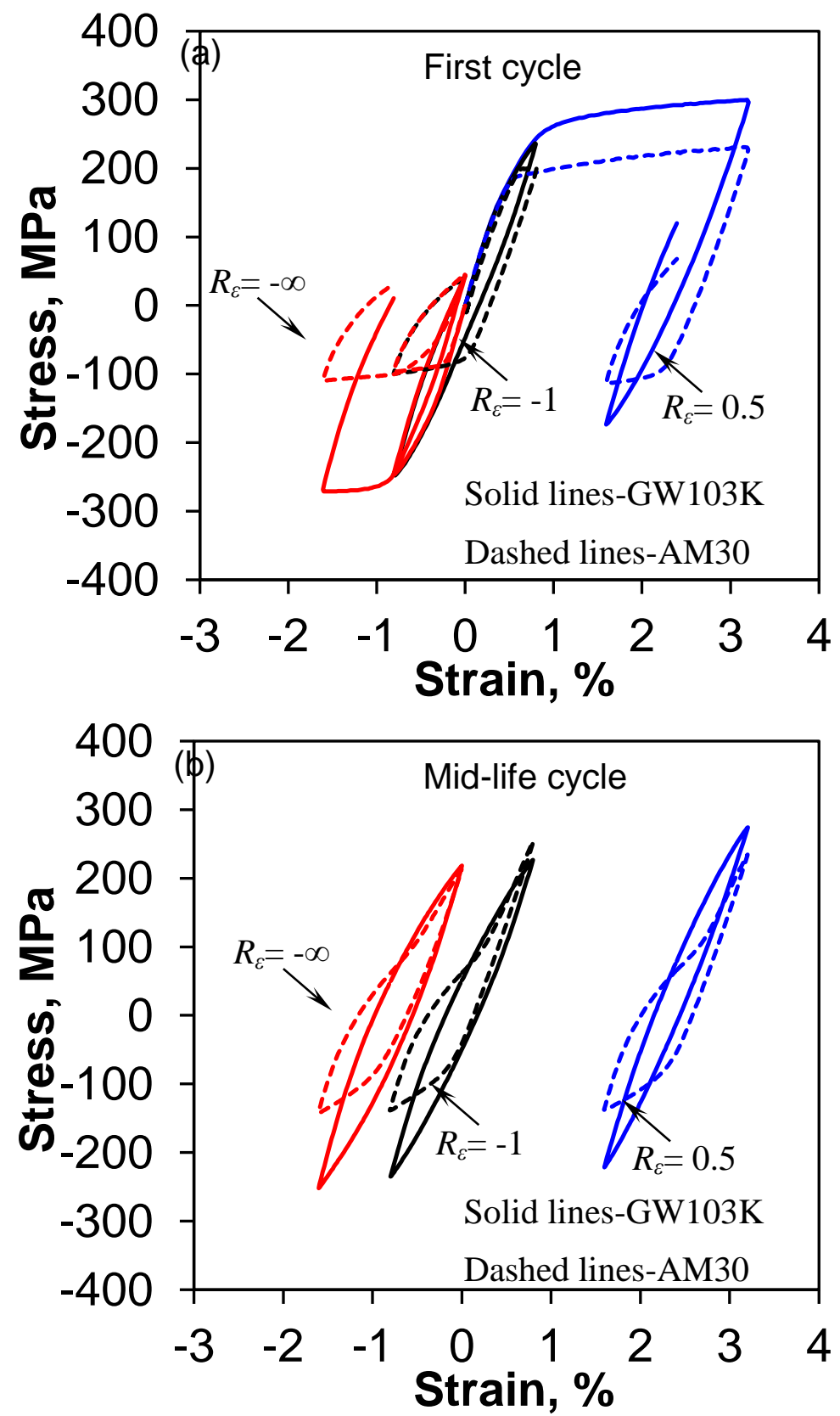

Figure 7.10: Typical stress-strain hysteresis loops of (a) first and (b) mid-life cycles at different strain ratios at a total strain amplitude of $0.8 \%$ in the GW103K alloy (solid lines) and AM30 alloy (dashed lines). 
This was mainly due to the microalloying with RE elements which led to significant changes in the texture and twinning activities arising from the fine grain size and especially RE-containing precipitates, which was discussed in section 6.5.2.
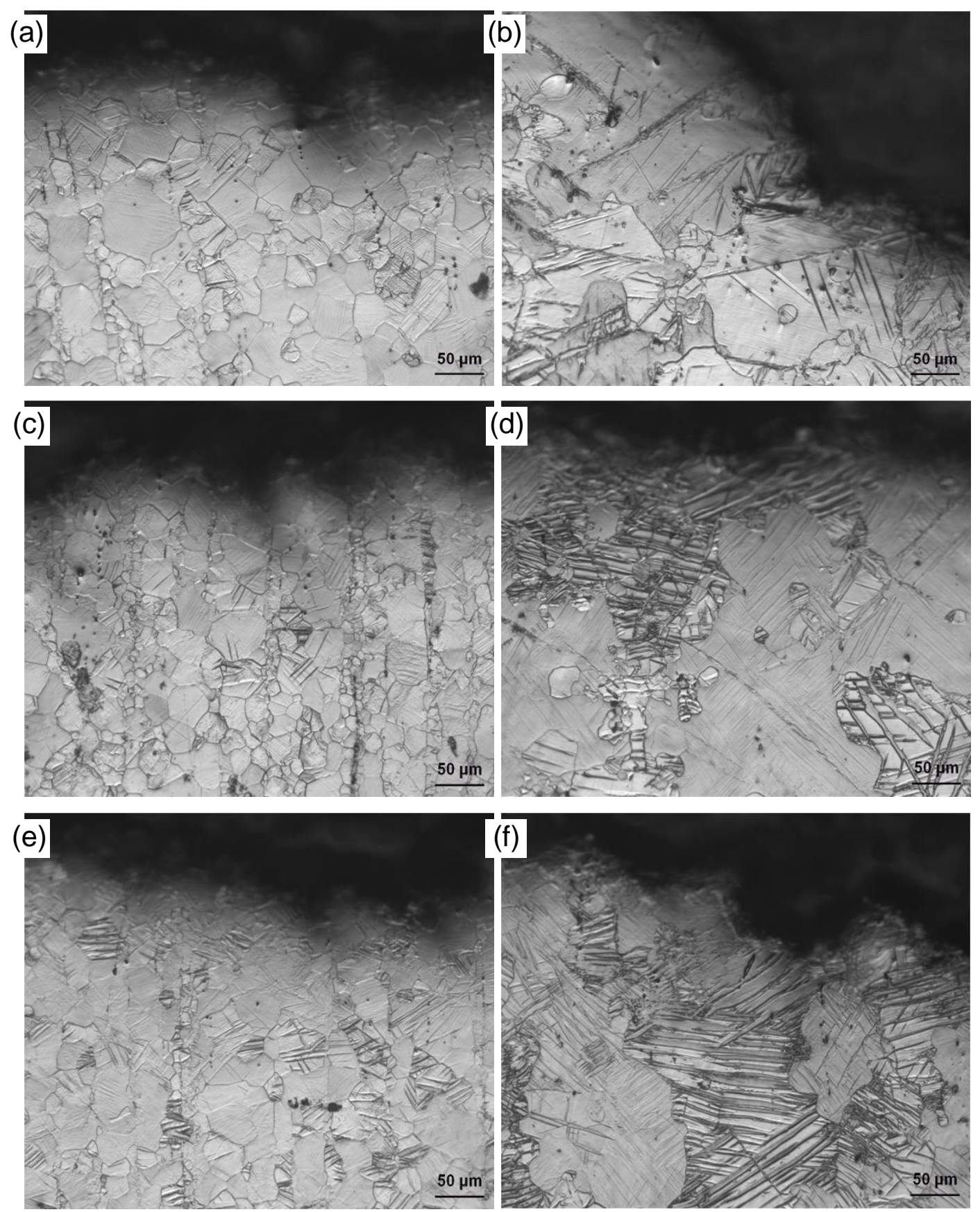

Figure 7.11: Optical micrographs in the areas near the fracture surface at a strain amplitude of 0.8\%, showing the distribution of residual twins in the fatigued samples of GW103K alloy ((a) 
$R_{\varepsilon}=0.5$, (c) $R_{\varepsilon}=-1$, and (e) $R_{\varepsilon}=-\infty$ ) and AM30 alloy (b) $R_{\varepsilon}=0.5$, (d) $R_{\varepsilon}=-1$, and (f) $\left.R_{\varepsilon}=-\infty\right)$, respectively.

\subsection{Summary}

The following conclusions can be drawn from this investigation:

1. At a strain ratio of $R_{\varepsilon}=0$ and 0.5 , a large amount of plastic deformation occurred in the tensile phase of the first cycle of hysteresis loops due to the high positive mean strain values. With decreasing strain ratio, the hysteresis loops became progressively wider.

2. The GW103K alloy exhibited essentially cyclic stabilization until failure at all strain ratios even at a fairly high strain amplitude of $0.8 \%$, indicating a beneficial effect of adding RE elements. Plastic strain amplitude was also stabilized until failure at all strain ratios, except a sudden drop from the first cycle to the second cycle. The plastic strain amplitude decreased with increasing strain ratio at a given strain amplitude and strain rate.

3. The fatigue life of the GW103K alloy was observed to be the longest in the fully reversed strain control at $R_{\varepsilon}=-1$, and it decreased as the strain ratio was deviated from $R_{\varepsilon}=-1$, due to the increasingly larger mean stress. As cyclic deformation progressed, a certain extent of mean stress relaxation occurred in the non-fully reversed strain control (i.e., $R_{\varepsilon} \neq-1$ tests). 


\title{
CHAPTER 8
}

\section{CYCLIC DEFORMATION BEHAVIOR OF AN EXTRUDED NZ30K}

\begin{abstract}
ALLOY
While the high performance of the GW103K alloy, especially in the aspect of suppressing the unwanted tension-compression asymmetry, was achieved (Chapter 6 and 7), this alloy was pretty expensive due to the addition of a fairly high amount (totally $\sim 13$ wt.\%) of RE elements. In the automotive applications cost is one of the major considerations. This has driven the development of relatively low $\mathrm{RE}$-containing $\mathrm{Mg}$ alloys, e.g., recently developed $\mathrm{Mg}-3 \mathrm{Nd}-0.2 \mathrm{Zn}-0.5 \mathrm{Zr}$ (NZ30K) alloy. This NZ30K alloy offers high strength and ductility as well as good creep resistance, it is thus very attractive to automotive powertrain and structural applications [233]. However, it is unclear to what extent the NZ30K $\mathrm{Mg}$ alloy would exhibit the tensioncompression asymmetry, whether cyclic hardening would occur, and what are the cyclic stress response and fatigue life. This part of the dissertation was, therefore, aimed at identifying cyclic deformation behavior of the low RE containing extruded NZ30K alloy, and evaluating the fatigue life under varying strain amplitudes.
\end{abstract}

\subsection{Microstructure}

Fig. 8.1 shows typical optical micrographs and SEM back-scattered electron images of NZ30K alloy in the as-extruded condition taken at different magnifications. The NZ30K alloy showed a bimodal microstructure due to the occurrence of dynamic recrystallization (DRX) in the hot extrusion process at $400^{\circ} \mathrm{C}$, which consisted of equiaxed recrystallized grains and 
unrecrystallized coarse grains. A significant number of larger intermetallic compounds were mainly present along the grain boundaries in the form of dashed lines, and a large portion of these coarse intermetallic phases were broken into small particles and moved from grain boundaries to grain interiors during the hot extrusion process, as indicated by arrows in Fig. 8.1(a). Similar types of microstructures were also reported in extruded $\mathrm{Mg}-2.4 \mathrm{Nd}-0.6 \mathrm{Zn}-0.6 \mathrm{Zr}$ alloy by Wen et al. [234] and extruded Mg-3Nd-0.2Zn-Zr alloy by Ma et al. [235]. The average grain size was approximately 5-7 $\mu \mathrm{m}$ which was fairly small in comparison with the common extruded Mg alloys, such as AZ31 and AM30 [25-28,35]. This was due to the role of added RE elements and zirconium ( $\mathrm{Zr}$ ) where $\mathrm{Zr}$ mainly restricted the grain growth [95]. A similar role of grain refinement by other RE elements, e.g., gadolinium [168], yttrium [169], cerium [170], neodymium [44,171], has also been reported. Besides, as shown in Fig. 8.1(a) no twins were present in the un-deformed samples. Fig. 8.1(b) shows a typical SEM back-scattered electron images of the as-extruded sample where a lot of larger particles along the grain boundaries in the form of dashed lines and smaller particles within the grains could be better seen. Furthermore, it can be seen more clearly from Fig. 8.1(c) and (d) taken at a higher magnification that there were some regions in the vicinity of grain boundaries which were free of precipitates - a phenomenon referred to as the "precipitate free zone" (PFZ). Similar types of PFZs were also reported in AlZn-Mg alloys [236] and Al-4Cu alloys [237]. The basic difference was that the PFZs in the present alloy could be seen at a microscopic level under optical microscope, whereas the previous literature showed PFZs at a nanoscopic level using transmission electron microscopy (TEM). To better see the intermetallic compounds in the as-extruded alloy, EDS line scan was performed as shown in Fig. 8.2(a) and (b), where it showed Nd-containing particles. X-ray diffraction pattern obtained from the as-extruded NZ30K alloy as shown in Fig. 8.3 confirmed 
the presence of two types of Nd-containing particles. Besides the $\alpha$-Mg peaks, metastable $\mathrm{Mg}_{12} \mathrm{Nd}$ peaks (which was simulated by the suppression of $\mathrm{Mg}_{41} \mathrm{Nd}_{5}$ peaks as reported by Zhang et al. [238]) and $\mathrm{Mg}_{3} \mathrm{Nd}$ peaks were detected in the XRD pattern. A similar type of second phase $\left(\mathrm{Mg}_{12} \mathrm{Nd}\right)$ was also reported in other $\mathrm{Mg}-\mathrm{Nd}$ alloys, e.g., in a gravity cast $\mathrm{Mg}-\mathrm{yNd}-\mathrm{XZn}-\mathrm{Zr}$ alloy by $\mathrm{Fu}$ et al. [239], cast $\mathrm{Mg}-3 \mathrm{Nd}-0.2 \mathrm{Zn}-04 \mathrm{Zr}$ alloy by $\mathrm{Fu}$ et al. [240], and an extruded $\mathrm{Mg}-\mathrm{Nd}-$ $\mathrm{Zn}-\mathrm{Zr}$ alloy by $\mathrm{Yu}$ et al. [241].
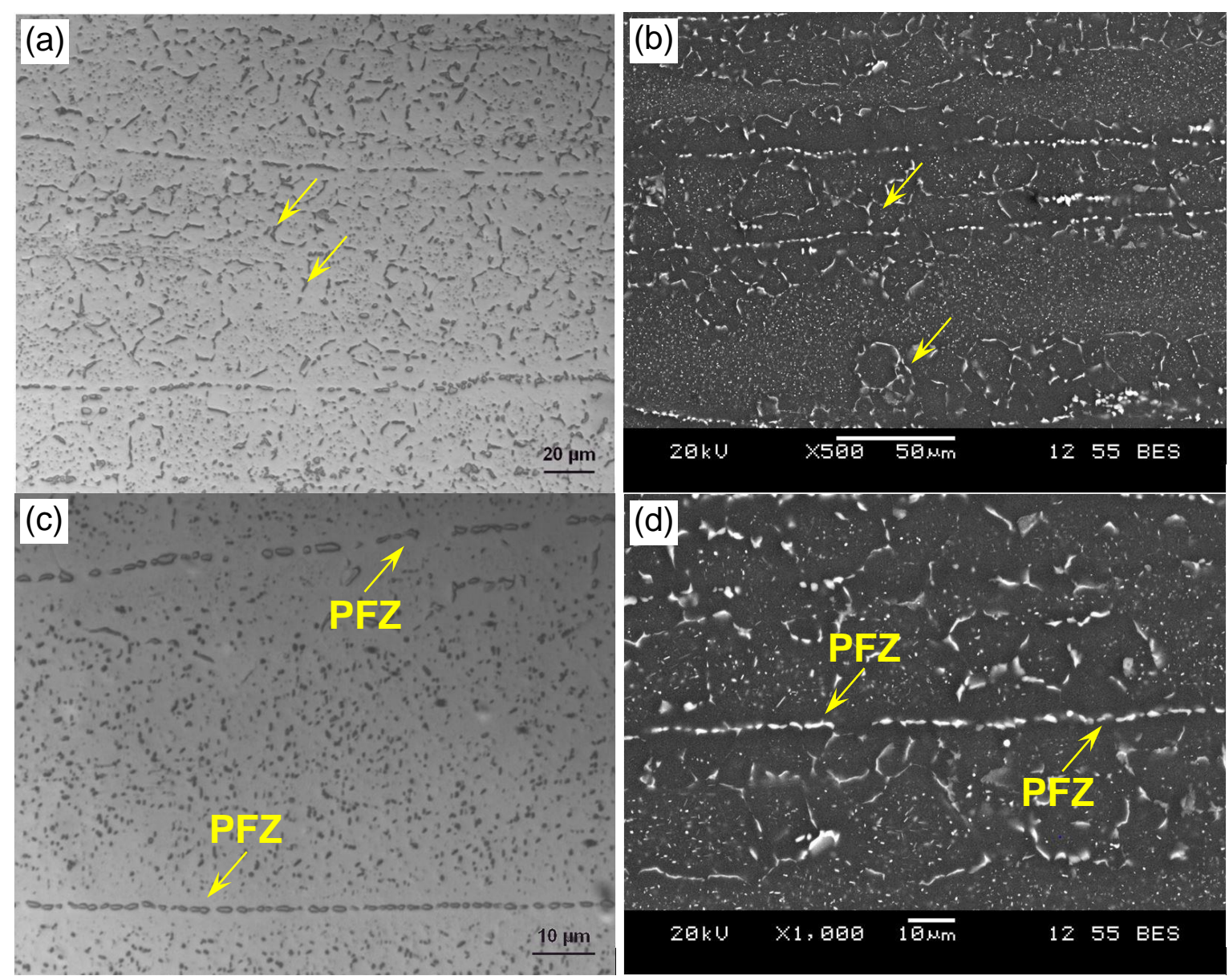

Figure 8.1: Microstructures of extruded NZ3OK alloy, (a) and (c) OM images, and (b) and (d) SEM back-scattered electron images taken at different magnifications. 
Furthermore, fine microstructures of as-extruded NZ30K alloy were also identified by three zone-axis electron microdiffraction patterns via TEM, as shown in Fig. 8.4. Electron microdiffration pattern in Fig. 8.4(b) shows the structure of the particle (A) (Fig. 8.4(a)) which was identified as cuboid-shaped (RE-rich) precipitates/particles (face-centered cubic (fcc) with $a=0.056 \mathrm{~nm}$ ). In addition, basal plane dislocation slip lines were also observed along $\langle 11-20\rangle_{\mathrm{Mg}}$ direction as indicated by an arrow in Fig. 8.4(a), which were formed during extrusion.
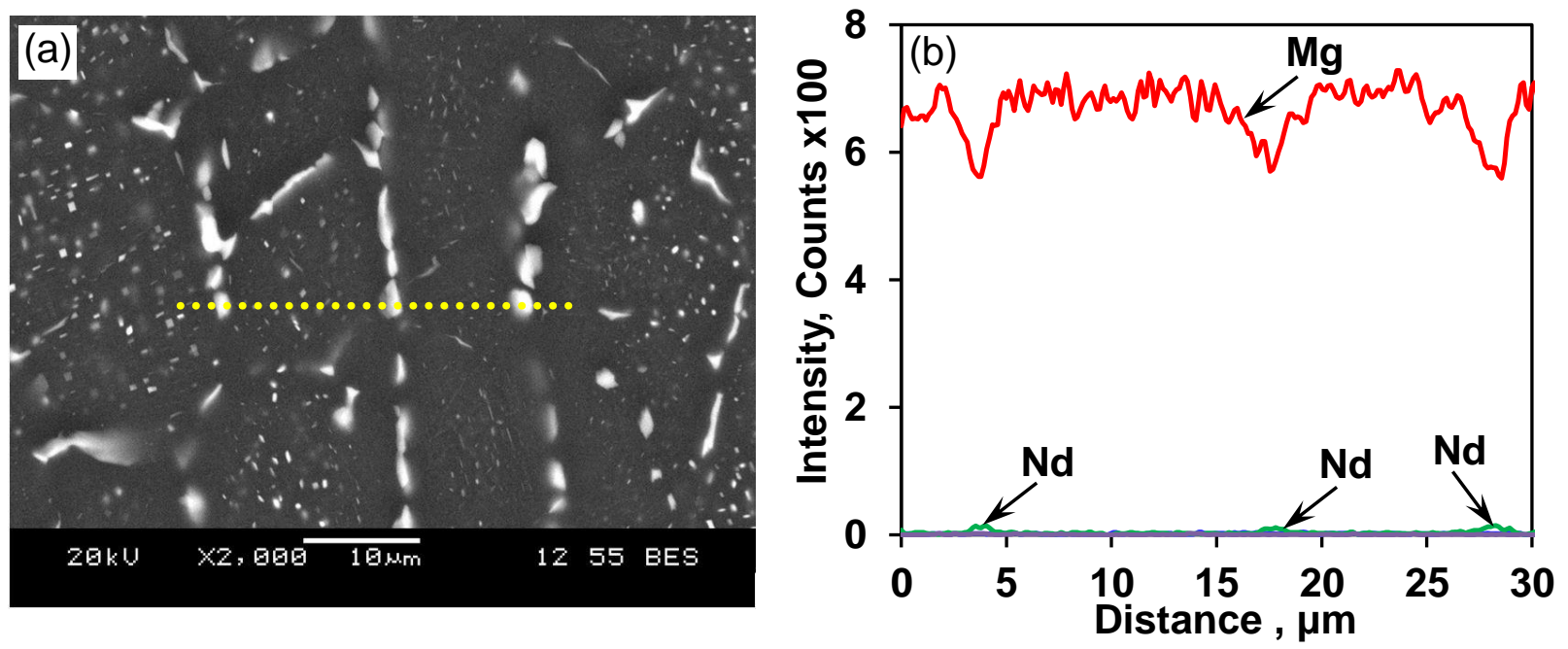

Figure 8.2: SEM back-scattered electron images indicating (a) EDS line scan position and (b) the corresponding EDS line scan results of NZ3OK alloy. 


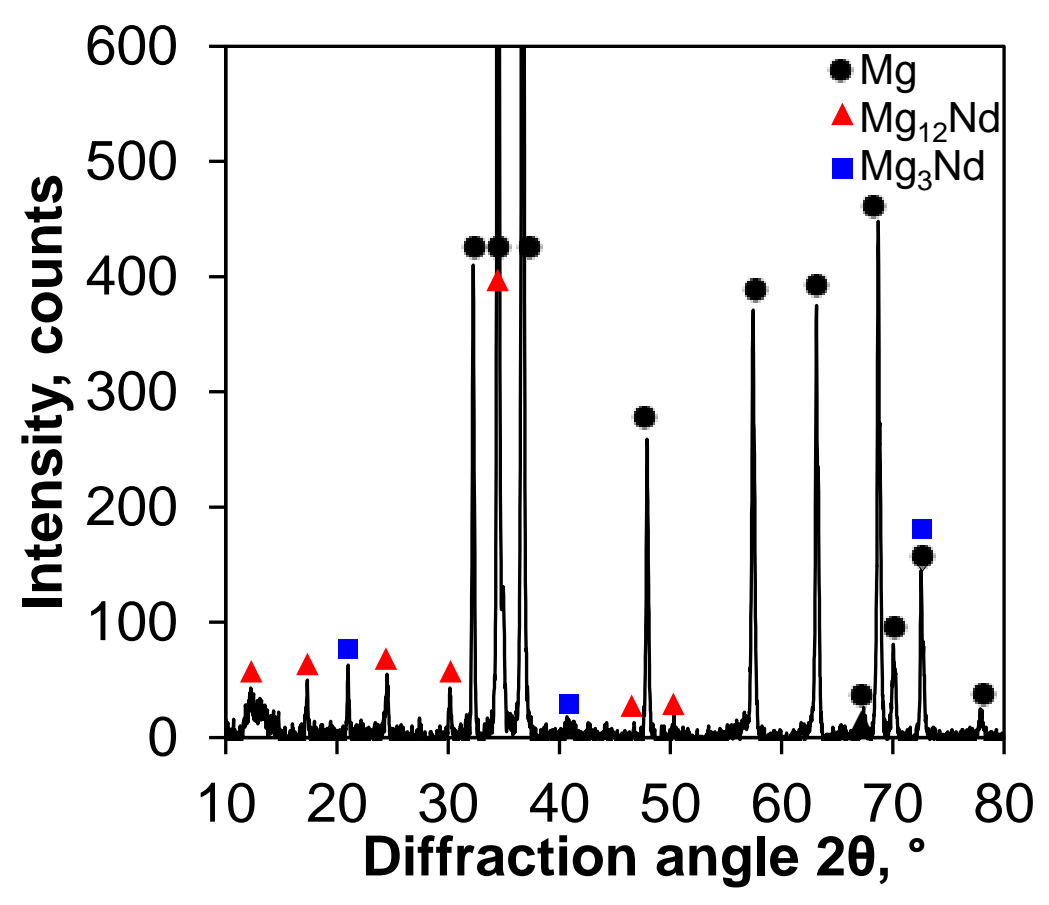

Figure 8.3: X-ray diffraction pattern of extruded NZ30K alloy.

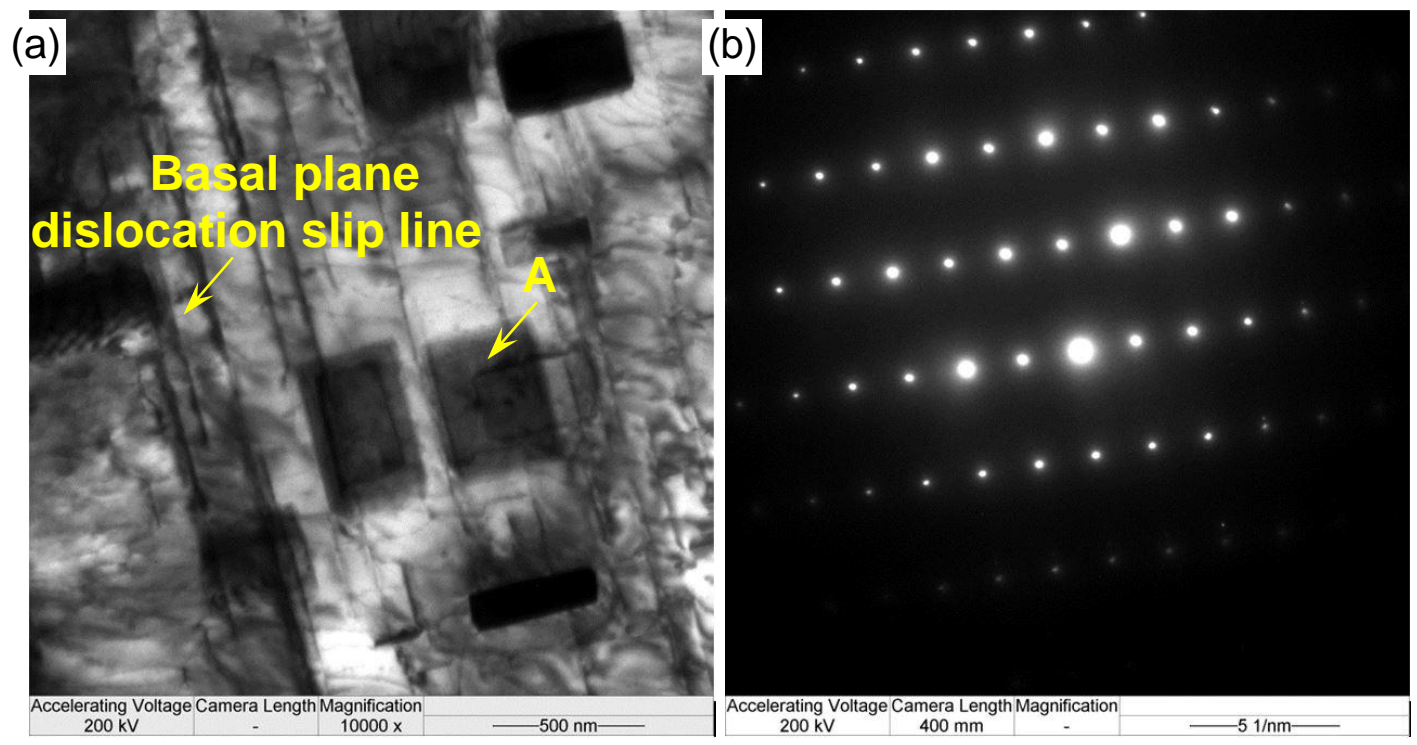

Figure 8.4: Transmission electron micrographs recorded from a sample of as-extruded NZ30K alloy: (a) bright-field image, and (b) corresponding SAED patterns, where beam line was parallel to $\langle 11-20\rangle_{M g}$. 


\subsection{Crystallographic texture}

Fig. 8.5 shows the initial crystallographic textures (pole figures of basal (0001) plane, prismatic

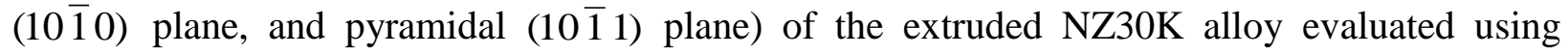
MTEX software where ED stands for the extrusion direction and RD indicates the radial direction. A relatively strong concentration (with a maximum intensity of 6.9 multiples of random distribution (MRD)) of basal (0001) pole mainly towards the $\mathrm{RD}$, along with prismatic $(10 \overline{1} 0)$ and pyramidal $(10 \overline{1} 1)$ poles towards the ED, was observed. This indicates that the $c$ axes of most grains were aligned perpendicular to the ED. While one benefit of the RE elements added into Mg alloys was to weaken the texture, e.g., Stanford and Barnett [60] reported that microalloying with RE elements could weaken texture in the forming process, it seemed that the texture in the extruded NZ30K alloy was not sufficiently weakened, in comparison with the reported results in other RE-Mg alloys [37, 42,46-50]. This could be related to the presence of PFZs (Fig. 8.1) and grainy $\mathrm{Mg}_{12} \mathrm{Nd}$ precipitates distributed in the intra-crystalline and along the grain boundaries as reported by Zheng et al. [242]. A similar higher texture intensity of NZ30K alloy has also been reported by Ma et al. [235].

\subsection{Tensile properties}

Fig. 8.6 shows the typical stress-strain curves obtained for the extruded NZ30K and GW103K alloys at a strain rate of $1 \times 10^{-4} \mathrm{~s}^{-1}$ at room temperature and the tensile properties obtained are listed in Table 8.1. As seen from Table 8.1, the present NZ30K alloy had a relatively lower strength and strain hardening exponent, but an equivalent or slightly higher ductility. In addition, 
the NZ30K alloy exhibited a certain degree of yield point phenomenon, in contrast to the smooth and continuous stress-strain curve of the GW103K alloy (Fig. 8.6). Similar yield point phenomenon on the tensile curve was also been reported for the NZ30K alloy by Ma et al. $[235,243]$. While being slightly inferior to the extruded GW103K alloy

due to a lower RE addition, the present NZ30K in the extruded state exhibited higher yield strength and ultimate tensile strength than the alloy in the cast state [239,240,244,245].

Figure 8.5: Pole figures of basal (0001) plane, prismatic (10 $\overline{1} 0)$ plane, and pyramidal (10 $\overline{1} 1)$

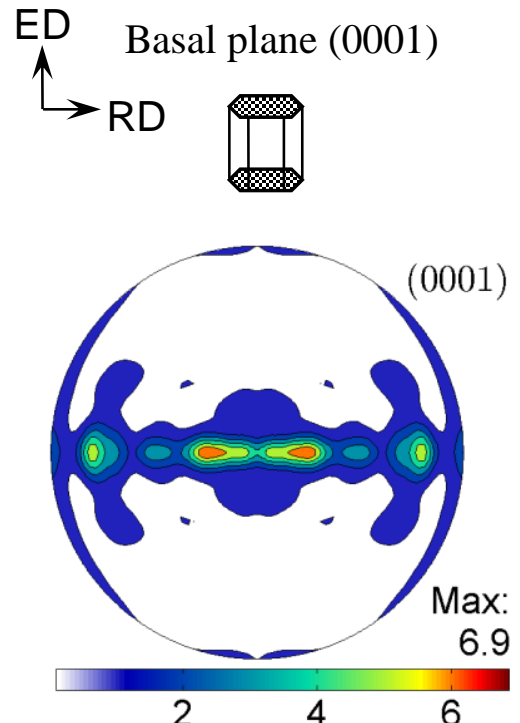

Prismatic plane $(10 \overline{1} 0)$

Pyramidal plane $(10 \overline{1} 1)$

2
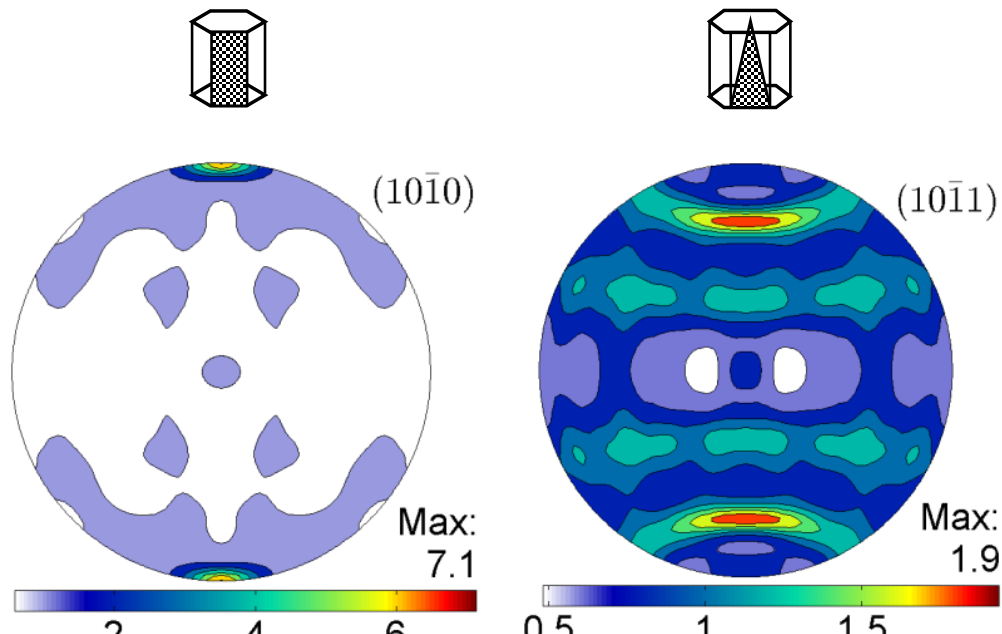

plane of extruded NZ3OK alloy, where ED stands for the extrusion direction and RD indicates the radial direction.

Table 8.1: Tensile properties of as-extruded NZ30K and GW103K alloys obtained at a strain rate of $1 \times 10^{-4} \mathrm{~s}^{-1}$.

\begin{tabular}{ccccc}
\hline Materials & $\sigma_{\mathrm{YS}, \mathrm{MPa}}$ & $\sigma_{\mathrm{UTS}}, \mathrm{MPa}$ & Elongation, \% & $n$ \\
\hline NZ30K & 180 & 225 & 9.1 & 0.12 \\
GW103K & 232 & 318 & 8.3 & 0.15 \\
\hline
\end{tabular}




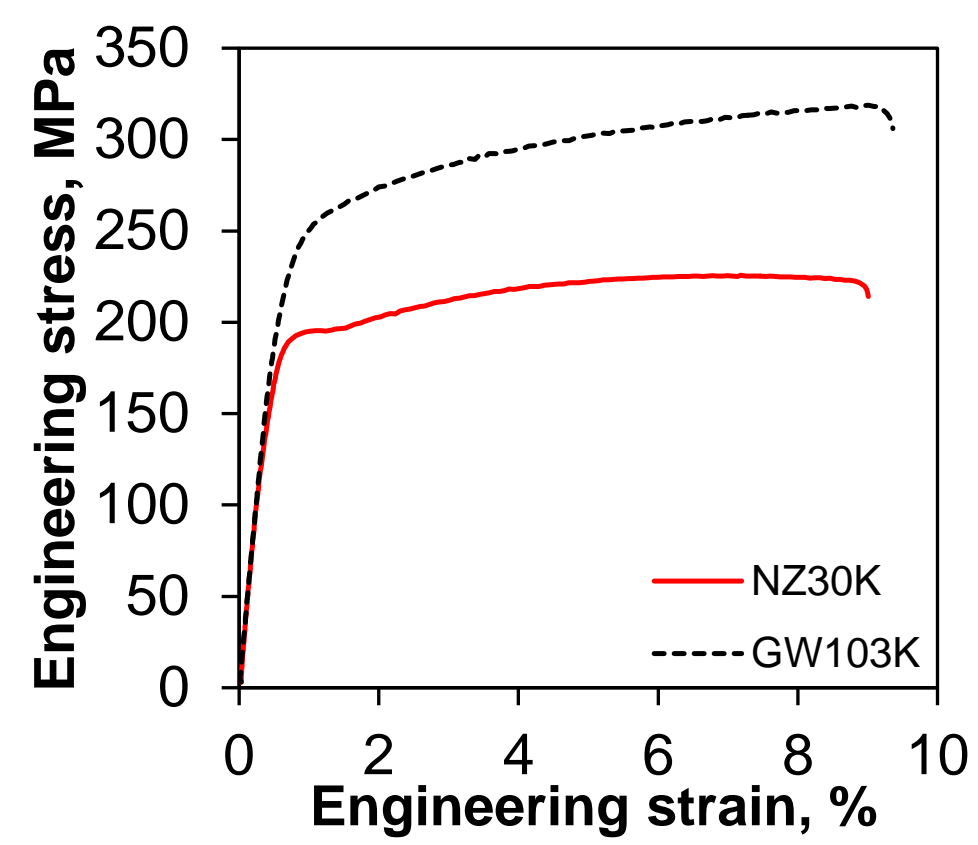

Figure 8.6: Typical tensile stress-strain curves of extruded NZ30K and GW103K alloys tested at a strain rate of $1 \times 10^{-4} \mathrm{~s}^{-1}$.

\subsection{Low cycle fatigue properties}

\subsubsection{Hysteresis loops}

Fig. 8.7 shows typical stress-strain hysteresis loops of the first, second, and mid-life cycles at a total strain amplitude of $1.2 \%$ and strain ratio of $R_{\varepsilon}=-1$ for the extruded NZ30K and AM30 alloys, respectively. It is seen from Fig. 8.7 that the initial slope in the ascending phase of the first cycle was the essentially same in both alloys, indicating that the 3\% RE addition did not significantly affect the Young's modulus. As seen from Fig. 8.7, unlike the extruded GW103K alloys (Fig. 6.6 and 6.14), the current NZ30K alloy exhibited asymmetrical hysteresis loops in shape, which were similar to those of RE-free Mg alloys [25-28,35,125,126]. This asymmetrical 
tensile and compressive yielding phenomenon was somewhat akin to the Bauschinger-like effect [34], which was predominantly associated with the twinning-detwinning process [25-28,129]. It is also seen from Fig. 8.7 that the very first cycle showed a stronger Bauschinger-like effect (i.e., a flatter curve in the compressive part in the descending phase) than the mid-life cycle. The possible reason behind this is that the formation of residual twins gradually became saturated near the mid-life cycle [28]. Such an obvious asymmetry or skewness of hysteresis loops in the present NZ30K alloy was mainly attributed to the existence of stronger crystallographic texture (Fig. 8.5), which caused the occurrence of extension twinning in compression and detwinning in tension during cyclic deformation along the ED. Similar twinning-detwinning activities in the extruded Mg alloys under cyclic loading were also reported in refs. [25-28,35]. In addition, unlike the fcc metals where the slope of hysteresis loops after the strain reversal either at the maximum or minimum stress was basically equal to the value of Young's modulus, the pseudoelastic or nonlinear elastic behavior in both descending and ascending phases in the NZ30K alloy was still present. It appeared reasonable to consider that the pseudoelastic behavior was an inherent characteristic of $\mathrm{Mg}$ alloys, which was in essence associated with the superior damping capacity.

\subsubsection{Cyclic deformation response}

Fig. 8.8 shows the evolution of stress amplitudes with respect to the number of cycles at different applied strain amplitudes on a semi-log scale. As the applied total strain amplitude decreased, the

stress amplitude decreased and the fatigue life of the alloy increased. Unlike the extruded GW103K alloy (Fig. 6.7) where microalloying with RE elements led to the cyclic stabilization at 
almost all the strain amplitudes, it seemed that cyclic stabilization was hardly achieved in the extruded NZ30K alloy as seen from Fig. 8.8. It exhibited a certain extent of cyclic softening at a low strain amplitude of $0.2 \%$. As the applied strain amplitude increased from $0.4 \%$ up to $1.0 \%$, the alloy showed basically cyclic stabilization within the first ten cycles and then cyclic hardening up to failure. At a higher strain amplitude of $1.2 \%$, an initial cyclic hardening within the first three cycles and then small cyclic softening could be seen from Fig. 8.8. Again, this would also be attributed to the twinning-detwinning activities of this NZ30K alloy, which will be presented later.

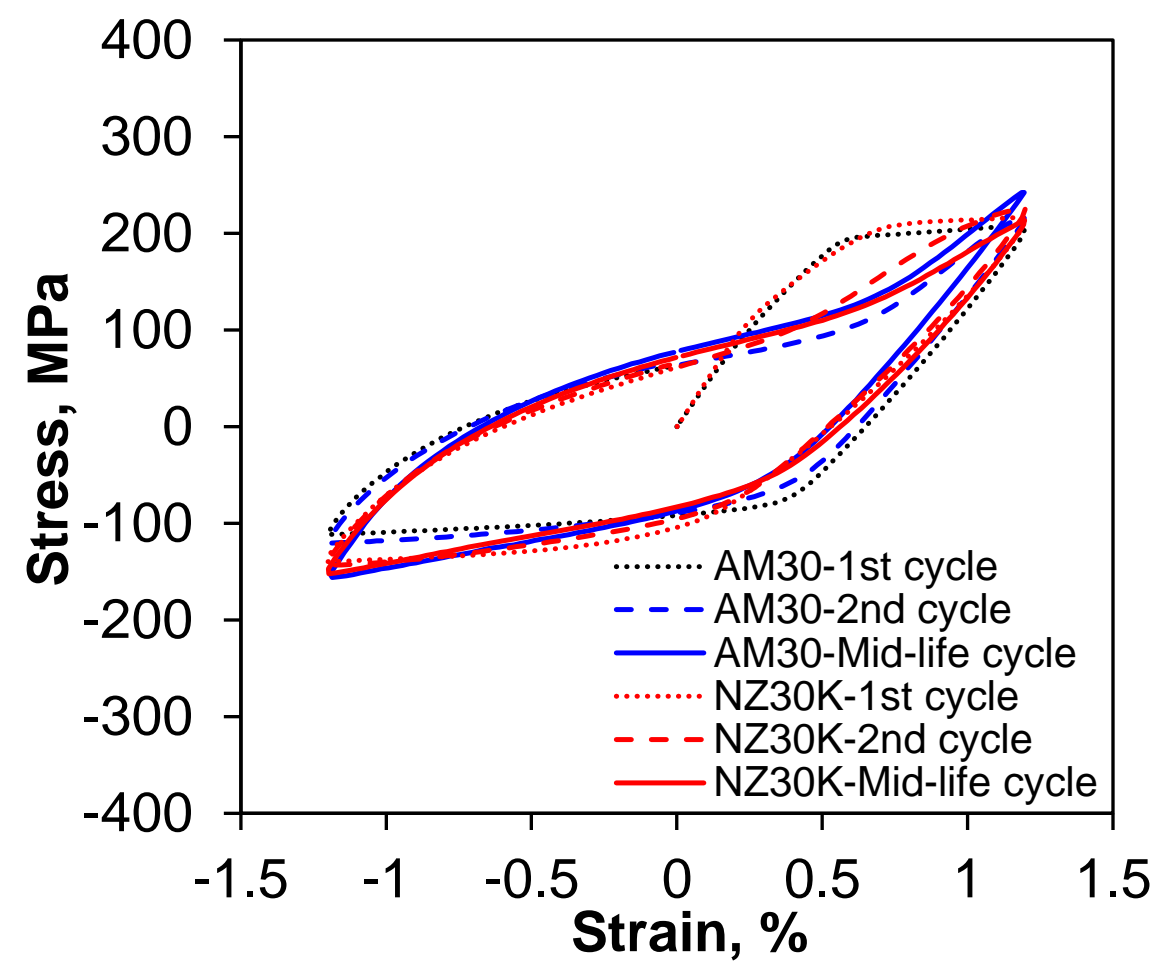

Figure 8.7: Typical stress-strain hysteresis loops of different cycles at a total strain amplitude of $1.2 \%$ and strain ratio of $R_{\varepsilon}=-1$ for the extruded AM30 and NZ3OK alloys, respectively. 


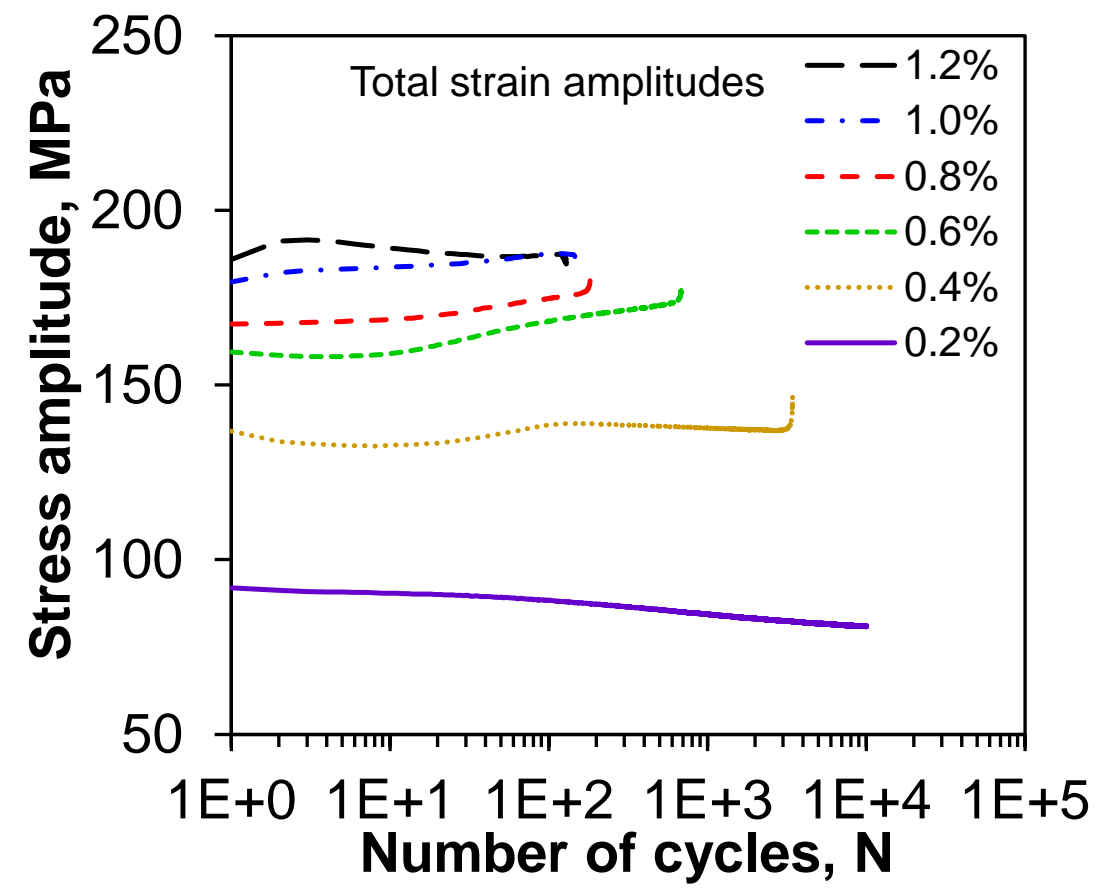

Figure 8.8: Stress amplitude vs. the number of cycles of the extruded NZ3OK alloy tested at different total strain amplitudes at a strain ratio of $R_{\varepsilon}=-1$.

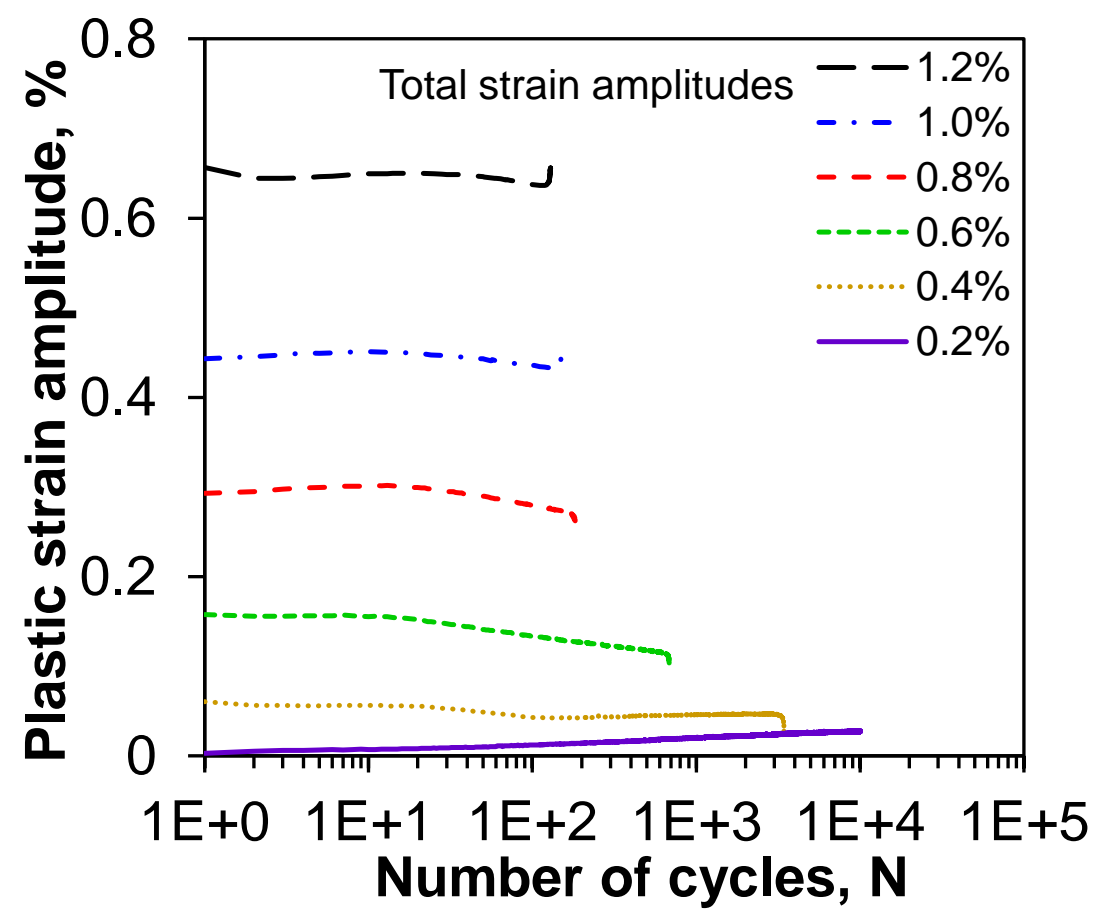

Figure 8.9: Plastic strain amplitude vs. the number of cycles of the extruded NZ30K alloy tested at different total strain amplitudes at a strain ratio of $R_{\varepsilon}=-1$. 
In general, cyclic response is governed by the cyclic stability of the microstructural features, dislocation glide and multiplication, twinning, and twin-dislocation interactions. The strain hardening phenomenon mainly arose from the interactions among the dislocations that impede the motion of dislocations [246]. As the basal texture in the $\mathrm{Mg}$ alloys facilitated $\{10 \overline{1} 2\}$ extension twinning, which caused a $86.3^{\circ}$ rotation of basal planes [16,247]. Such a change in the orientation would act as a barrier to the movement of dislocations on the original slip plane and caused the formation of dislocations pile-ups, which initiated the back stress and thus resulted in the strain hardening [28]. In the low cycle fatigue tests, plastic strain amplitude has been considered as a physical quantity that results in several damaging processes and influences the internal microstructure which is closely related to the strain resistance and eventually the fatigue life [26]. The change of the plastic strain amplitude $\left(\Delta \varepsilon_{\mathrm{p}} / 2\right)$ during cyclic deformation is shown in Fig. 8.9, which corresponded well to the change of the stress amplitude during cyclic deformation as shown in Fig. 8.8 at different total strain amplitudes.

\subsubsection{Fatigue life and fatigue parameters}

The fatigue life (i.e., the number of cycles to failure, $N_{f}$ ) as a function of the applied total strain amplitudes $\left(\Delta \varepsilon_{\mathrm{t}} / 2\right)$ of the extruded NZ30K alloy is plotted in Fig. 8.10, along with the experimental data reported in the literature for a variety of extruded $\mathrm{Mg}$ alloys [26,28,156-159] for comparison. Run-out data points were denoted by arrows pointing horizontally at or more than $10^{7}$ cycles. The alloy showed basically a similar fatigue life to that of the RE-free extruded Mg alloys within the experimental scatter [26,28,156-159]. 


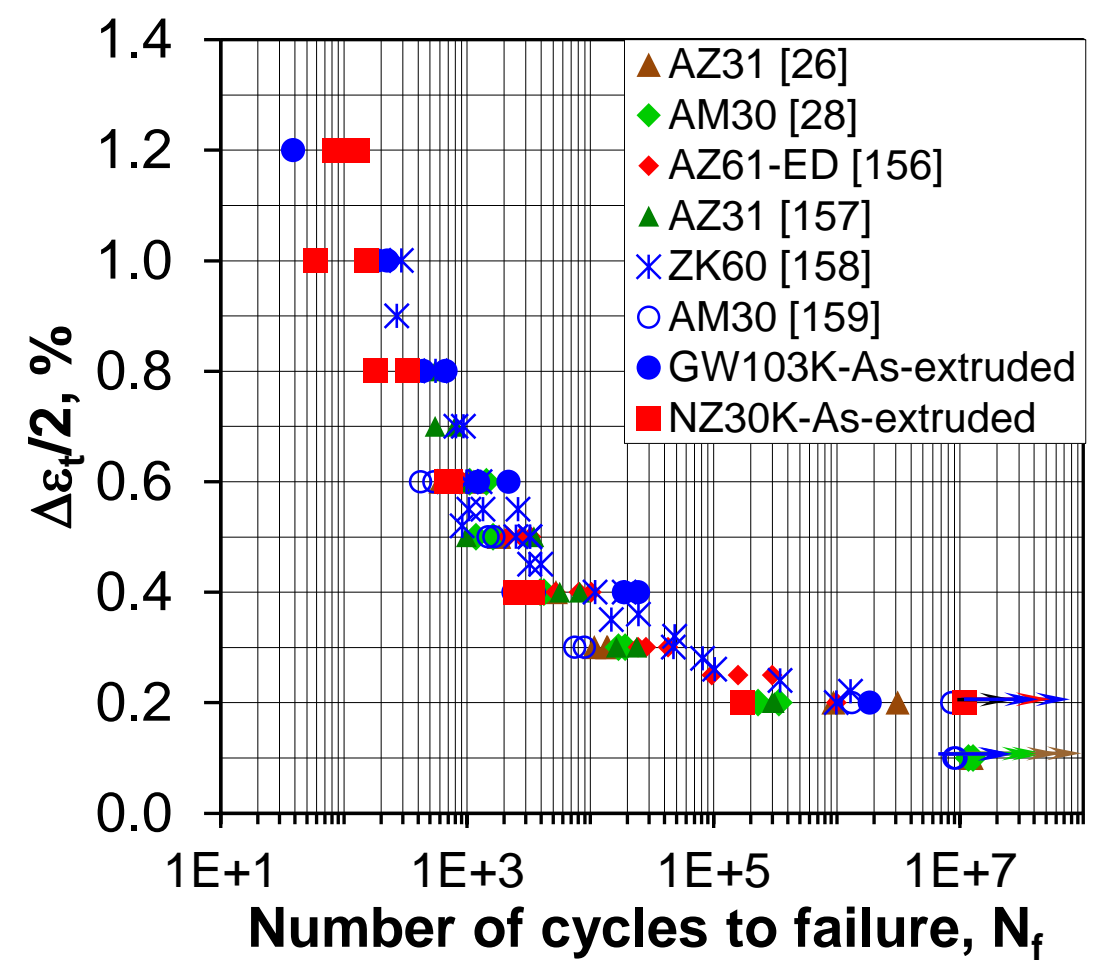

Figure 8.10: Total strain amplitude versus the number of cycles to failure for the extruded NZ3OK alloy, in comparison with the data reported in the literature for various extruded Mg alloys [26,28,156-159].

The fatigue life parameters evaluated on the basis of equs (6.1) and (6.2) are summarized in Table 8.2. It is seen that while the obtained fatigue parameters were well within the range in other fatigued $\mathrm{Mg}$ alloys reported in the literature [26-28,125,152,155], the cyclic strain hardening exponent $n^{\prime}$ of NZ30K alloy was lower than that of GW103K alloy (Table 6.2). This corresponded well to the lower monotonic strain hardening exponent $n$ (Table 8.1) and cyclic softening characteristics of this NZ30K alloy (Figs 8.8 and 8.9). Fig. 8.11 shows the elastic, plastic, and total strain amplitudes plotted as a function of the number of reversals to failure $\left(2 N_{f}\right)$ in a double-log scale. It should be noted that in evaluating the above fatigue life parameters, the run-out data for the fatigue samples without failure at or above $10^{7}$ cycles were not included. 
Furthermore, the obtained cyclic stress-strain curve corresponding to the mid-lives of the NZ30K alloy tested at varying strain amplitudes is shown in Fig. 8.12 along with the monotonic stressstrain curve. It is seen that the cyclic stress-strain curve was positioned slightly lower than the monotonic one of the extruded NZ30K alloy. The obtained cyclic yield strength $\left(\sigma_{y}^{\prime}\right)$ and cyclic strain hardening exponent $\left(n^{\prime}\right)$ of the NZ30K alloy were also slightly lower than the corresponding monotonic yield strength and strain hardening exponent, as seen from Tables 8.1 and 8.2. This could be the reason why the NZ30K alloy exhibited a certain extent of cyclic softening at a low strain amplitude of $0.2 \%$.

Table 8.2: Low cycle fatigue parameters obtained for the as-extruded NZ3OK alloy.

\begin{tabular}{cc}
\hline Low cycle fatigue parameters & Extruded NZ30K \\
\hline Cyclic yield strength, $\sigma_{y}^{\prime}, \mathrm{MPa}$ & 165 \\
Cyclic strain hardening exponent, $n^{\prime}$ & 0.10 \\
Cyclic strength coefficient, $K^{\prime}, \mathrm{MPa}$ & 313 \\
Fatigue strength coefficient, $\sigma_{f}^{\prime}, \mathrm{MPa}$ & 431 \\
Fatigue strength exponent, $b$ & -0.10 \\
Fatigue ductility coefficient, $\varepsilon_{f}^{\prime}$ & 0.24 \\
Fatigue ductility exponent, $c$ & -0.72 \\
\hline
\end{tabular}




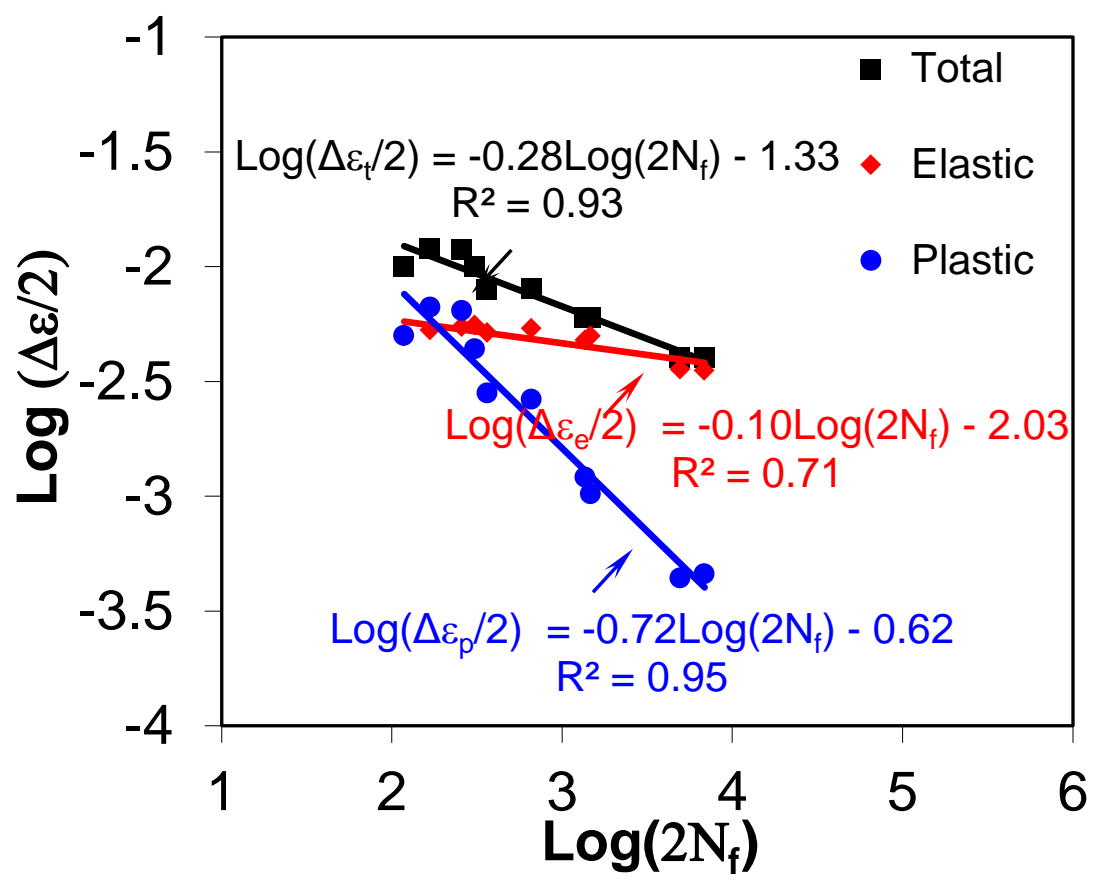

Figure 8.11: Cyclic total, elastic, and plastic strain amplitudes vs. number of reversals to failure of the extruded NZ3OK alloy.

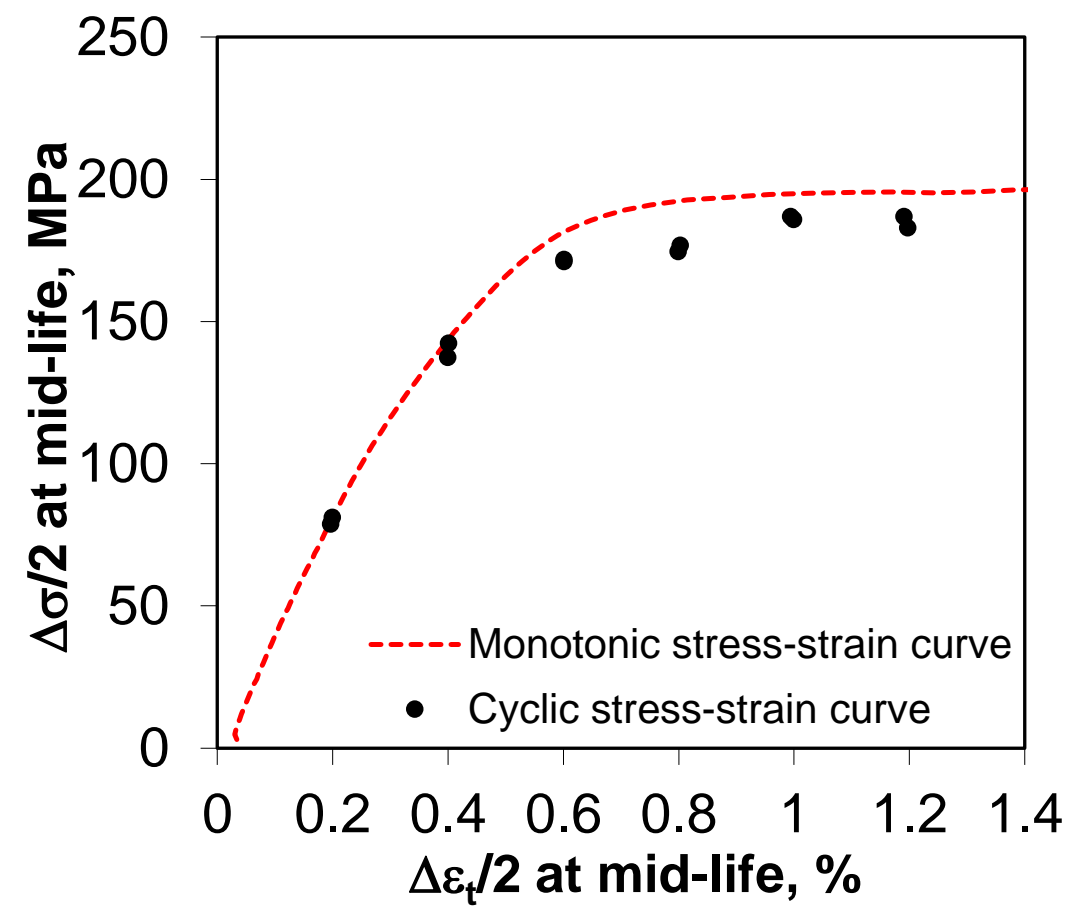

Figure 8.12: Cyclic stress-strain curve for extruded NZ3OK alloy, where the corresponding monotonic stress-strain curve is plotted for comparison. 


\subsubsection{Fractography}

Fig. 8.13 shows an overall view of typical fracture surfaces at a lower magnification for the specimens tested at a strain amplitude of $0.4 \%$ and $1.0 \%$, respectively. It is seen that fatigue crack initiated basically from the specimen surface. On a close examination at a higher magnification (Fig. 8.14), fatigue crack indeed initiated from near-surface defects and the fracture surfaces at both strain amplitudes had similar features, i.e., isolated facet of the cleavage-like planes near the initiation site. However, the fatigue crack propagation characteristics of extruded NZ30K alloy in both strain amplitudes were quite different from those of GW103K alloy (Figs 6.11 and 6.12), which were basically characterized by serrated fatigue striations observed at higher magnifications (Fig. 8.15). It is known that the occurrence of fatigue striations was due to a repeated plastic blunting-sharpening process in face-centered cubic (fcc) materials stemming from the slip of dislocations in the plastic zone ahead of the fatigue crack tip [224]. The formation of fatigue striations in the HCP alloy was anticipated to be related to both dislocation slip and twinning in the plastic zone during fatigue crack propagation [25-28]. It should be noted that the fatigue striations were perpendicular to the crack propagation direction. A similar type of fracture surfaces was reported in a cast $\mathrm{Mg}-3 \mathrm{Nd}-0.2 \mathrm{Zn}-0.5 \mathrm{Zr}$ alloy by $\mathrm{Li}$ et al. [233]. Furthermore, a visible PFZ was seen from the back-scattered electron images (Figs 8.15(b), (d) and (f)). 

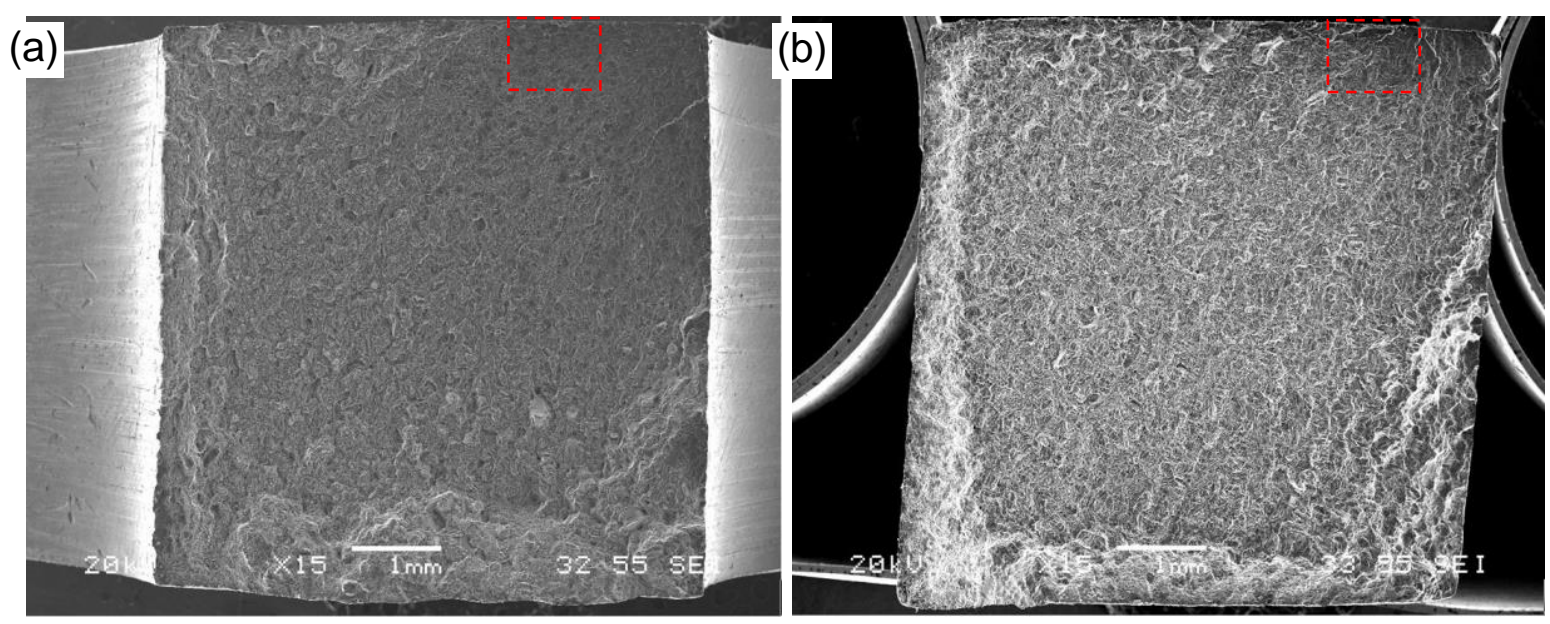

Figure 8.13: SEM images of overall fracture surfaces of the extruded NZ3OK specimens fatigued at a total strain amplitude of (a) $0.4 \%$ and $(b) 1.0 \%$.
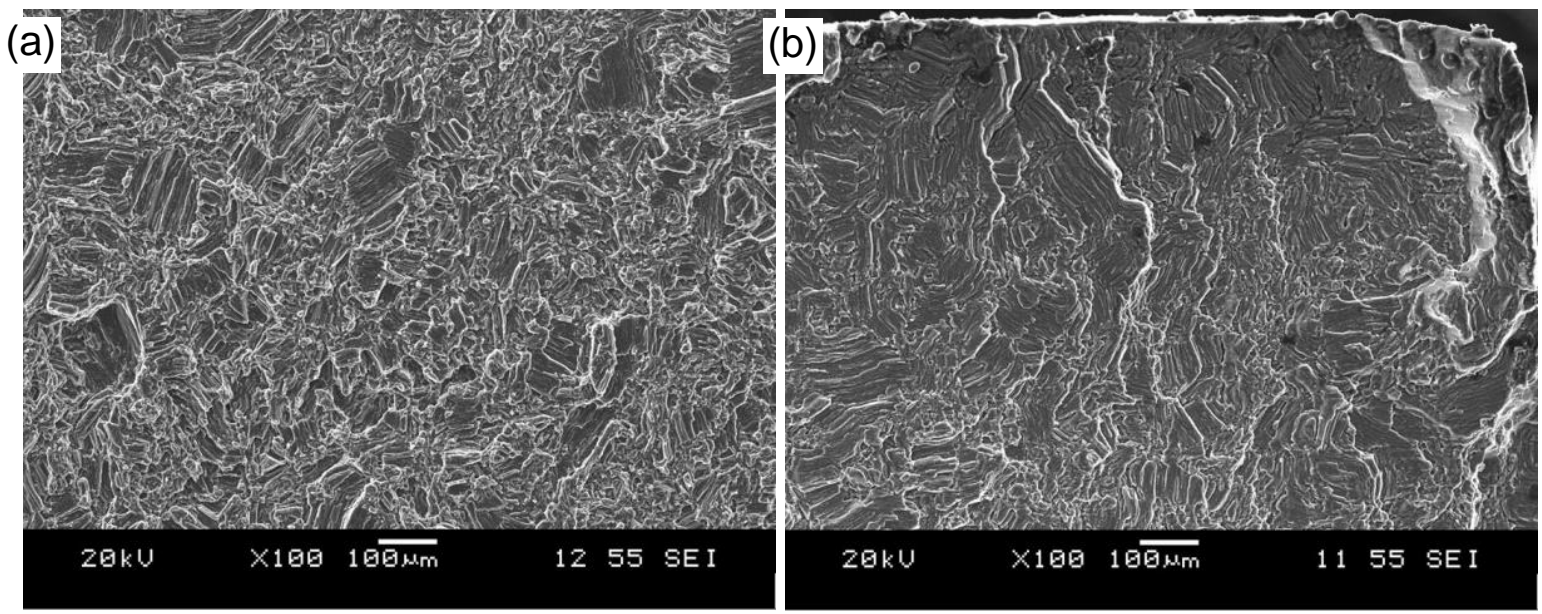

Figure 8.14: SEM micrographs of the fracture surface near crack initiation of the extruded NZ30K specimens fatigued at a total strain amplitude of (a) $0.4 \%$, and (b) $1.0 \%$. 

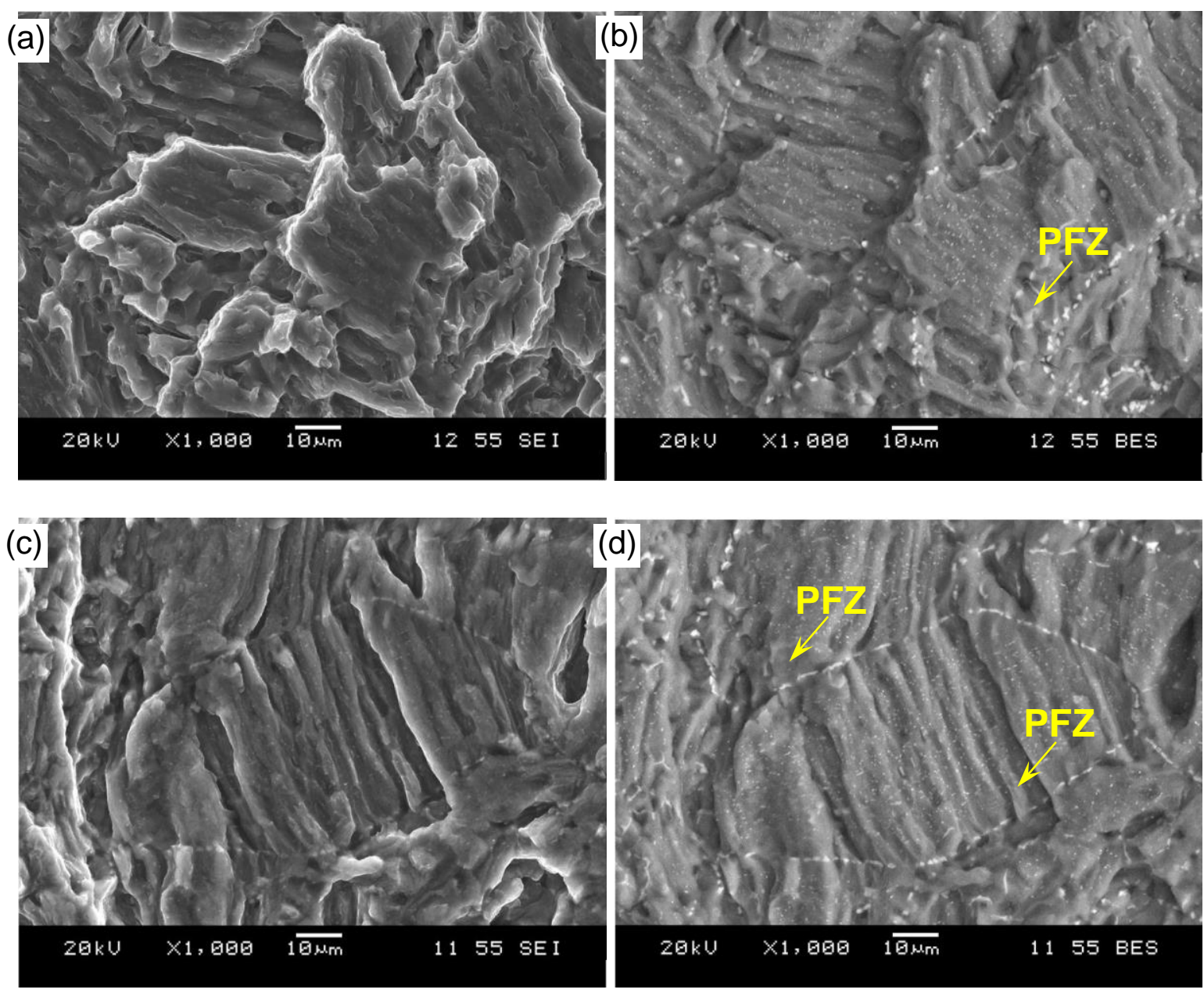

Figure 8.15: SEM micrographs (left: SEM images and right: SEM back-scattered electron images) of the fatigue crack propagation region of the extruded NZ3OK specimens fatigued at a total strain amplitude of (a) and (b) $0.4 \%$ and $(c)$ and $(d) 1.0 \%$.

\subsection{Discussion}

In polycrystalline alloys, the mechanical properties are directly related to the presence of crystallographic textures which dominate the orientation of slip or twinning planes and directions relative to the externally applied stress, thus affect the yield asymmetry between tension and 
compression $[16,182,229]$. Since $\{10 \overline{1} 2\}$ extension twinning is a key deformation mechanism in Mg alloys [25-28,248], the combination of twinning and crystallographic textures in wrought $\mathrm{Mg}$ alloys is mainly responsible for the tension-compression yield asymmetry which is commonly observed in nearly all RE-free wrought Mg alloys [16,25-28,38-40]. It was also reported by Jain et al. [229] that the yield asymmetry is sensitive to textures. As presented earlier in section 8.2, the texture of the extruded NZ30K alloy was not sufficiently weakened by the addition of RE elements (Fig. 8.5) as compared to the GW103K alloy (Fig. 6.4), along with the presence of PFZs (Fig. 8.1) and grainy $\mathrm{Mg}_{12} \mathrm{Nd}$ precipitates distributed in the intra-crystalline and along the grain boundaries. The basic difference between the NZ30K alloy and GW103K was the amount of RE elements, i.e., only $\sim 3$ wt. $\%$ Nd vs. $\sim 10$ wt.\% Gd and $\sim 3$ wt.\% Y. As the RE content increased, the texture sharpness generally decreased [115]. The differences in both the type and amount of RE elements were thus expected to have a strong influence on the texture formation which plays an important role in the yield asymmetry of the NZ30K alloy (Fig. 8.7). To better see the difference, the stress-strain hysteresis loop of the mid-life cycle of the extruded NZ30K alloy is re-plotted in Fig. 8.16, in conjunction with that of both RE-free Mg alloy (AM30) and RE-containing GW103K alloy. It can be clearly seen that the hysteresis loop of NZ30K alloy exhibited a strong skewed asymmetrical shape similar to that of the RE-free AM30 alloy, unlike GW103K alloy (Fig. 8.16). The ratio of the compressive-to-tensile yield stress was $\sim 0.55$ for both AM30 and NZ30K alloys, as compared to the GW103K alloy where the ratio was almost 1. This can again be attributed to the lower amount of RE element in NZ30K alloy. On the other hand, as seen from Fig. 8.17 which shows the distribution of deformation twins in the area near the fracture surface in the fatigued samples of AM30, GW103K and NZ30K alloys, respectively, the grain size of the RE-containing alloys (NZ30K and GW103K) was smaller than that of the 
RE-free AM30 alloy (Fig. 8.17(b), (c) vs. (a)). However, in the extruded NZ30K alloy the smaller grain size did not seem to affect the hysteresis loops considerably, as compared with the GW103K alloy (Fig. 6.1). The most striking feature of the NZ30K alloy was the presence of PFZs along the grain boundaries (as shown in Figs 8.1, 8.15, and 8.17). This, in conjunction with the absence of a high density of nano-sized and bamboo-leaf/plate-shaped $\beta^{\prime}\left(\operatorname{Mg}_{7}(\mathrm{Gd}, \mathrm{Y})\right)$ precipitates (Fig. 6.3), increased the tendency of twinning in the NZ30K alloy as indicated by the dense residual twins near the fracture surface area (Fig. 8.17(c)), which subsequently led to the asymmetric tension-compression hysteresis loops (Figs 8.7 and 8.16).

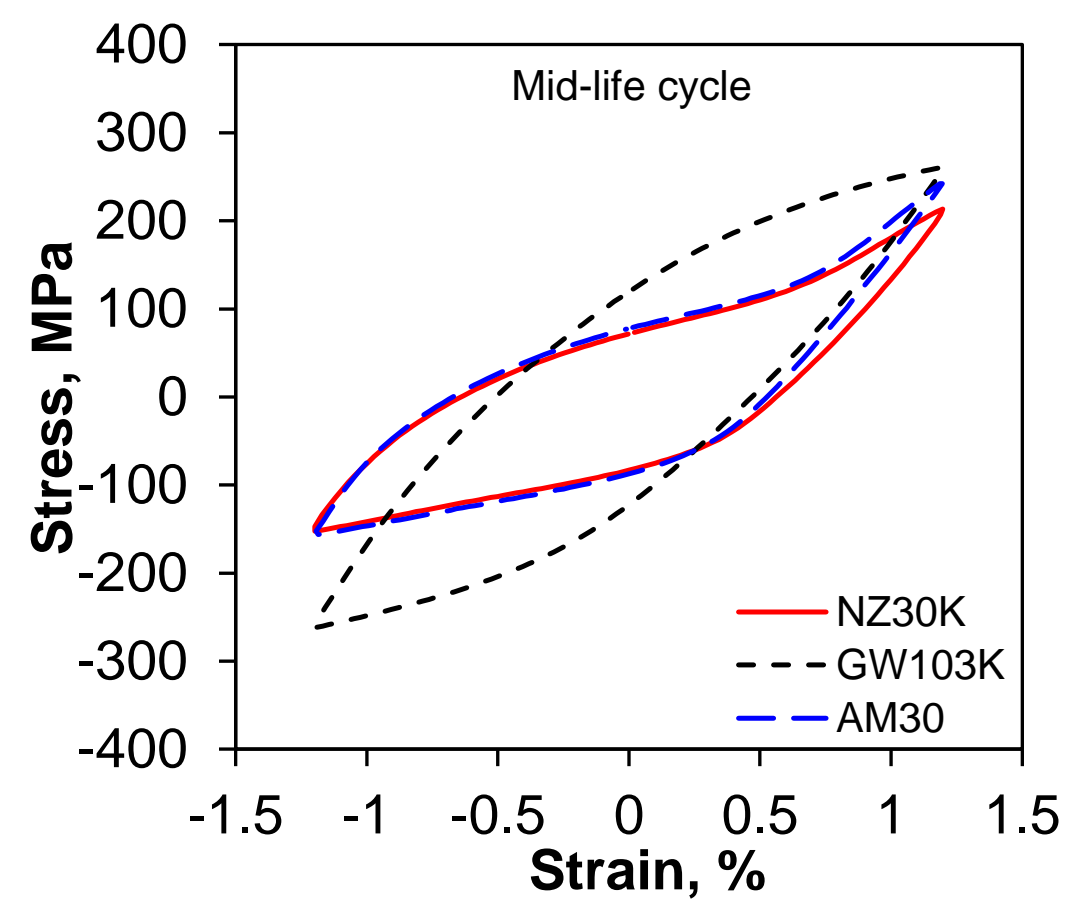

Figure 8.16: Typical stress-strain hysteresis loops of the mid-life cycle at a given total strain amplitude of $1.2 \%$ and strain ratio of $R_{\varepsilon}=-1$ for the extruded NZ30K, GW103K, and AM30 alloys, respectively. 

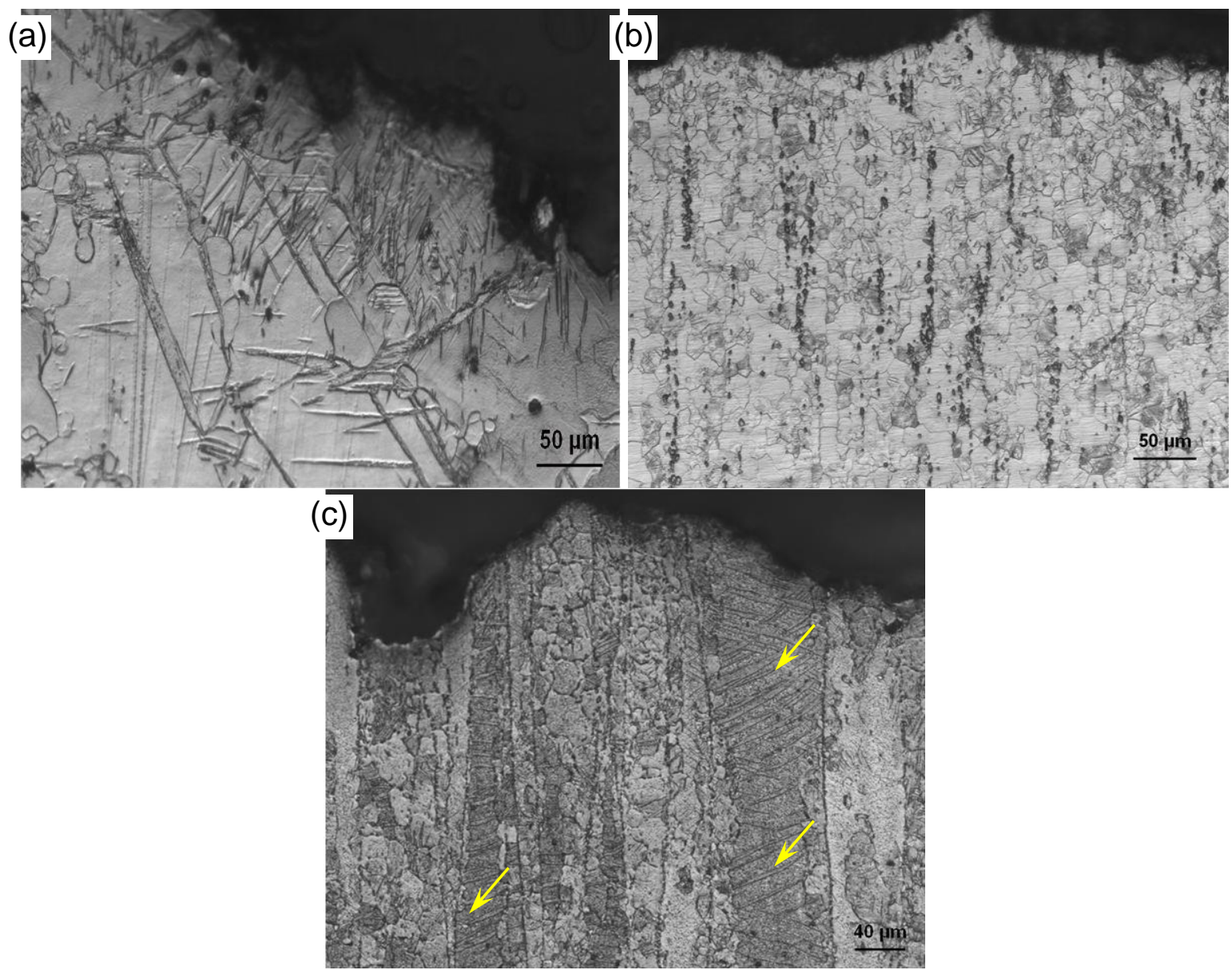

Figure 8.17: Optical micrographs in the area near the fracture surface at a strain amplitude of $1.2 \%$, showing the distribution of deformation twins in the fatigued samples of (a) AM30, (b) asextruded $G W 103 K$, and (c) as-extruded NZ30K alloys, respectively.

The above results indicated that the effect of $3 \mathrm{wt} \% \mathrm{Nd}$ on the overall cyclic deformation behavior of the NZ30K alloy was not so strong. The change in the deformation behavior, as compared with the high RE-containing GW103K alloy, was mainly related to (i) the presence of PFZs (Fig. 8.1), (ii) grainy $\mathrm{Mg}_{12} \mathrm{Nd}$ precipitates distributed in the intra-crystalline and along the grain boundaries (Fig. 8.1), and (iii) the stronger texture (Fig. 8.5). The coupled role of these factors was responsible for the hysteresis loop asymmetry (Figs 8.7 and 8.16), the decrease in the 
stress amplitude (Fig. 8.8) and the increase in the plastic strain amplitude at a given total strain amplitude (Fig. 8.9), while a similar fatigue life was obtained among the NZ30K, and AM30 alloys within the experimental scatter.

\subsection{Summary}

Strain-controlled low cycle fatigue tests were conducted on an extruded NZ30K alloy with different total strain amplitudes at room temperature. The following key points can be drawn from this investigation:

1. The microstructure of the NZ30K alloy consisted of a bimodal microstructure with equiaxed recrystallized grains and unrecrystallized coarse grains with an average grain size of about approximately 5-7 $\mu \mathrm{m}$. A large number of smaller second-phase particles were present inside the grains and larger particles along the grain boundaries accompanied by the presence of characteristic precipitate free zone (PFZ).

2. Unlike the higher RE-containing GW103K alloy, the NZ30K alloy exhibited basically asymmetrical hysteresis loops. This was predominantly due to the presence of the relatively stronger crystallographic texture with $c$-axes perpendicular to the extrusion direction, which promoted heavily the twinning-detwinning activities during cyclic deformation.

3. The cyclic stabilization was barely achieved even at the lower strain amplitudes. The extruded NZ30K alloy exhibited cyclic softening at low strain amplitudes and cyclic hardening at high strain amplitudes. However, the fatigue life of this alloy was observed 
to be equivalent to that of other RE-free extruded Mg alloys, which could be described by the Coffin-Manson law and Basquin's equation.

4. SEM examinations revealed that fatigue cracks initiated from the near-surface defects and the initiation site contained isolated facet of the cleavage-like planes. The propagation was basically characterized by serrated fatigue striations. The PFZ was further confirmed from the back-scattered electron images. 


\section{CHAPTER 9}

\section{SUMMARY, CONCLUSIONS AND FUTURE WORK}

\subsection{Summary}

In this dissertation, monotonic and cyclic deformation behavior of rare-earth containing extruded $\mathrm{Mg}$ alloys were investigated, with special attention to the effect of the RE element addition on the twin formation. First of all, in Chapter 4, the deformation behavior of an extruded GW103K alloy in compression along the extrusion direction at different strain levels was investigated, and the experimental results regarding the extent of twinning during compression and the effects of twinning or detwinning on the strain hardening were presented and discussed. To understand better the deformation behavior of RE-Mg alloys, i.e., to describe the flow behavior of RE-Mg alloys based on the more commonly-used tensile deformation tests, a modified constitutive equation was proposed for the GW103K alloy based on the well-known and highly-cited ${ }^{3}$ Johnson-Cook model and a recently-proposed strain hardening equation in Chapter 5. Comparisons between the predicted and experimental results demonstrated that the modified Johnson-Cook constitutive equation was able to estimate the flow stresses for the GW103K alloy fairly accurately with a standard deviation of about $1.83 \%$.

As the principal goal of this dissertation was to explore the cyclic deformation behavior of RE$\mathrm{Mg}$ alloys, strain-controlled low cyclic fatigue tests (LCF) were conducted at different strain

\footnotetext{
${ }^{3}$ References [199] and [201] about the Johnson-Cook model have been cited 2684 and 1844 times, respectively, based on a Google search on August 14, 2014.
} 
amplitudes and at different strain ratios (Chapters 6-8). In Chapter 6, the results from the fully reversed strain-controlled fatigue tests of extruded GW103K alloy in different heat treatment states under varying strain amplitudes were reported and a significant decrease of twinningdetwinning activities was observed which directly led to a reduction of the unfavorable or unwanted tension-compression yield asymmetry of stress-strain hysteresis loops, and an improved fatigue life as compared to the conventional extruded wrought $\mathrm{Mg}$ alloys. Also, the cyclic stress response was much more stable in the RE-containing GW103K alloy. In Chapter 7, the low cycle fatigue behavior of extruded GW103K alloy with emphasis on the effect of strain ratio was demonstrated. To achieve the aim of this chapter the low cycle fatigue tests were performed using different strain ratios at a constant amplitude of $0.8 \%$ and a strain rate of $1 \times 10^{-2}$ $\mathrm{s}^{-1}$. The results obtained confirmed that the strain ratio had a significant effect on the cyclic deformation characteristics of the extruded GW103K alloy. For instance, due to the high positive mean strain values, a large amount of plastic deformation occurred in the tensile phase which could be observed from the hysteresis loops. The fatigue life of the GW103K alloy was observed to be the longest in the fully reversed strain control at $R_{\varepsilon}=-1$, and it decreased as the strain ratio was deviated from $R_{\varepsilon}=-1$, due to the increasingly larger mean stress.

In Chapter 8, to identify the effect of the amount (e.g., lower than 10wt.\%) and type of RE elements (Nd, rather than $\mathrm{Gd}$ or $\mathrm{Y}$ ) on the cyclic deformation and fatigue life, fully reversed strain-controlled fatigue tests of a recently-developed $\mathrm{Mg}-3 \mathrm{Nd}-0.2 \mathrm{Zn}-0.5 \mathrm{Zr}$ (NZ30K) alloy under varying strain amplitudes were reported. The results obtained indicated that the cyclic deformation behavior of NZ30K alloy was fairly different from that of the GW103K alloy. The alloy exhibited basically asymmetrical hysteresis loops due to the presence of the relatively 
strong crystallographic texture which promoted heavily the twinning-detwinning activities during cyclic deformation. The fatigue life of the NZ30K alloy was observed to be similar to the RE-free extruded Mg alloys.

\subsection{Conclusions}

The main conclusions of this dissertation could be summarized as follows:

I. A significant beneficial role of grain refinement has been observed in both RE-Mg GW103K and NZ30K alloys. The microstructure of GW103K alloy consisted of uniform equiaxed grains due to the occurrence of dynamic recrystallization (DRX) in the hot extrusion process.

On the other hand, the NZ30K alloy consisted of a bimodal microstructure with equiaxed recrystallized grains and unrecrystallized coarse grains. A large number of smaller particles were present inside the grains and larger particles along the grain boundaries alongside the precipitate-free zone in the NZ30K alloy.

II. RE-containing particles were present in the GW103K alloy in all the three conditions (i.e., as-extruded, T5, and T6 heat treated). After T6 heat treatment, the RE-containing particles contained more $\mathrm{Zr}$ and had a more rounded shape. In particular, a high density of nano-sized and bamboo-leaf/plate-shaped $\beta^{\prime}\left(\mathrm{Mg}_{7}(\mathrm{Gd}, \mathrm{Y})\right)$ precipitates were observed to distribute uniformly in the $\alpha-\mathrm{Mg}$ matrix.

III. Microalloying of Mg with RE elements can weaken the basal texture. Relatively weak basal fiber textures were formed in GW103K alloys regardless of the heat treatment states, where the $c$-axes of most grains were aligned perpendicular to the ED. However, depending on the 
amount of RE elements added, the texture sharpness was different, since the NZ30K alloy exhibited a fairly strong concentration of basal pole density.

IV. Three stages of strain hardening were observed in the as-extruded GW103K alloy during compression: (1) A rapidly decreasing strain hardening rate up to a strain level of $\sim 4 \%$ (stage A), (2) a slightly decreasing and almost linear strain hardening rate over an extended strain range from $\sim 4 \%$ to $\sim 18 \%$ (stage B), and (3) a rapidly decreasing strain hardening rate (stage C) just prior to failure.

V. The amount of twins observed in the as-extruded GW103K alloy was much less extensive than that in the RE-free extruded Mg alloys due to the weaker crystallographic texture, refined grain size, and RE-containing second-phase particles.

VI. A modified Johnson-Cook constitutive equation was proposed, which was able to estimate the flow stresses for the GW103K alloy fairly accurately with a standard deviation of about $1.83 \%$.

VII. Unlike the RE-free Mg alloys, GW103K alloy exhibited basically symmetrical hysteresis loops in tension and compression, regardless of alloy states (i.e., in the extruded, T5 and T6 heat-treated conditions), suggesting the absence of the tension-compression yield asymmetry. However, NZ30K alloy exhibited basically asymmetrical hysteresis loops which were predominantly due to the twinning-detwinning activities during cyclic deformation.

VIII. The GW103K alloy in different states exhibited cyclic stabilization until failure, whereas the cyclic stabilization was barely achieved even in the lower strain amplitudes in the extruded NZ30K alloy. This was related to the amount of RE elements addition. 
IX. The fatigue lives of GW103K alloy were longer than that of the RE-free Mg alloys, and could be well described by the Coffin-Manson law and Basquin's equation. However, the fatigue life of NZ30K was observed to be equivalent to that of the RE-free Mg alloys.

X. SEM examinations revealed that fatigue crack initiated from the specimen surface or nearsurface defects in both alloys, and the initiation site contained some cleavage-like facets. The propagation was basically characterized by fatigue striations, in conjunction with some secondary cracks.

XI. The strain ratio had a significant effect on the cyclic deformation characteristics of the GW103K alloy. The GW103K alloy exhibited essentially cyclic stabilization until failure at all strain ratios even at a fairly high strain amplitude of $0.8 \%$, indicating a beneficial effect of adding RE elements.

XII. The fatigue life of the GW103K alloy was observed to be the longest in the fully reversed strain control at $R_{\varepsilon}=-1$, and it decreased as the strain ratio was deviated from $R_{\varepsilon}=-1$, due to the increasingly larger mean stress. As cyclic deformation progressed, a certain extent of mean stress relaxation occurred in the non-fully reversed strain control (i.e., $R_{\varepsilon} \neq-1$ tests).

\subsection{Recommendations for Future Work}

Traditionally, the yielding, work hardening and fracture behavior of metals are primarily characterized using uniaxial testing. The anisotropic behavior of wrought $\mathrm{Mg}$ alloys requires that 
a much wider range of loading paths be examined to provide adequate information for predicting the behavior of $\mathrm{Mg}$ alloys so as to guarantee the safety, reliability and durability of structural components. The present investigation was aimed at studying the mechanical properties and fatigue behavior of different RE-Mg alloys. While a number of results have been obtained in the present study, there still remained a lot of work that needs to be further done. The following points are the recommendations for the future work:

I. Bi-axial or multi-axial fatigue tests for the RE-Mg alloys would be an interesting aspect to study the anisotropic behavior of these alloys both in tensile and fatigue modes, since no such results have been reported in the literature for these alloys yet.

II. In the LCF, once the crack initiates, it would propagate quickly due to the high stress level, while in the $\mathrm{HCF}$, the crack propagation would consume most of the fatigue life. It is thus of interest to study the fatigue crack growth behavior and the related propagation mechanisms for these RE-Mg alloys.

III. Further detailed microstructural characterization of the RE-Mg alloys with or without heat treatment, e.g., twin boundary structures, twin-dislocation and twin-precipitate interactions at an atomic level, needs to be done using high-resolution TEM, high-temperature XRD, and if possible using neutron diffraction as well.

IV. There were a number of reports describing the appearance and disappearance of twins (also known as twinning-detwinning phenomenon) by observing in-situ fatigue deformation in some wrought $\mathrm{Mg}$ alloys via neutron diffraction. It would be of great interest to carry out insitu examinations of the RE-Mg alloys to study the texture formation, twinning-detwinning behavior and also internal stresses redistribution during cyclic tests. 
V. It would be very helpful to predict the cyclic deformation behavior of RE-Mg alloys using finite element analysis (FEA) method, along with a quantitative correlation between the strain hardening, twinning, and texture of RE-Mg alloys. 


\section{REFERENCES}

[1] Gillett, N. P., Arora, V. K., Zickfeld, K., Marshall, S. J., and Merryfield, W. J. (2011) Ongoing climate change following a complete cessation of carbon dioxide emissions. Nature Geoscience, 4, 83-87.

[2] Schiermeier, Q. (2014) Water risk as world warms. Nature, 505, 10-11.

[3] McNutt, M. (2013) Climate change impacts. Science, 341, 435.

[4] Ash, C., Culotta, E., Fahrenkamp-Uppenbrink, J., Malakoff, D., Smith, J., Sugden, A., and Vignieri, S. (2013) Once and future climate change. Science, 341, 473.

[5] Shakun, J. D., Clark, P. U., He, F., Marcott, S. A., Mix, A. C., Liu, Z. Y., Otto-Bliesner, B., Schmittner, A., and Bard, E. (2012) Global warming preceded by increasing carbon dioxide concentrations during the last deglaciation. Nature, 484, 49-54.

[6] Reichstein, M., Bahn, M., Ciais, P., Frank, D., Mahecha, M. D., Seneviratne, S. I., Zscheischler, J., Beer, C., Buchmann, N., Frank, D. C., Papale, D., Rammig, A., Smith, P., Thonicke, K., van der Velde, M., Vicca, S., Walz, A., and Wattenbach, M. (2013) Climate extremes and the carbon cycle. Nature, 500, 287-295.

[7] Min, S. K., Zhang, X. B., Zwiers, F. W., and Hegerl, G. C. (2011) Human contribution to more-intense precipitation extremes. Nature, 470 378-381.

[8] Schiermeier, Q. (2011) Increased flood risk linked to global warming. Nature, 470, 316.

[9] Pall, P., Aina, T., Stone, D. A., Scott, P. A., Nozawa, T., Hilberts, A. G. J., Lohmann, D., and Allen, M. R. (2007) Anthropogenic greenhouse gas contribution to flood risk in England and Wales in autumn 2000. Nature, 470 (7334), 382-385.

[10] Tang, Q. H., Zhang, X. J., and Francis, J. A. (2014) Extreme summer weather in northern mid-latitudes linked to a vanishing cryosphere. Nature Climate Change, 4, 45-50. 
[11] Pollock, T. M. (2010) Weight loss with magnesium alloys. Science, 328, 986-987.

[12] Nie, J. F., Zhu, Y. M., Liu, J. Z., and Fang, X. Y. (2013) Periodic segregation of solute atoms in fully coherent twin boundaries. Science, 340, 957-960.

[13] Murray, J. and King, D. (2012) Oil's tipping point has passed. Nature, 481(7382), 433435.

[14] Wise, M., Calvin, K., Thomson, A., Clarke, L., Lamberty, B. B., Sands, R., Smith, S. J., Janetos, A., and Edmonds, J. (2009) Implications of limiting $\mathrm{CO}_{2}$ concentrations for land use and energy. Science, 324, 1183-1186.

[15] Joost, W. J. (2012) Reducing vehicle weight and improving U.S. energy efficiency using integrated computational materials engineering. JOM, 64, 1032-1038.

[16] Mirza, F. A. and Chen, D. L. in: S. Zhang, D.L. Zhao (Eds.). (2013) Fatigue of lightweight magnesium alloys. Aerospace Materials Handbook, CRC Press, Taylor \& Francis, New York, 647-698.

[17] Liu, C., Chen, D. L., Bhole, S. D., Cao, X., and Jahazi, M. (2009) Polishing-assisted galvanic corrosion in the dissimilar friction stir welded joint of AZ31 magnesium alloy to 2024 aluminum alloy. Mater. Charact., 60(5), 370-376.

[18] Kainer, K. U. (2003) Magnesium - Alloys and Technology. Wiley-VCH, Cambridge.

[19] Mordike B. L. and Ebert, T. (2001) Magnesium: properties applications potential. Mater. Sci. Eng. A, 302(1), 37-45.

[20] Luo, A. A (2002) Magnesium: Current and potential automotive applications. JOM, 54, 42-48.

[21] Nyberg, E. A., Luo, A. A., Sadayappan, K., and Shi, W. F. (2008) Magnesium for future autos. Adv. Mater. Process., 166, 35-37. 
[22] Luo, A. A. (2004) Recent magnesium alloy development for elevated temperature applications. Int. Mater. Rev., 49(1), 13-30.

[23] Pantelakis, Sp. G., Alexopoulos, N. D., and Chamos, A. N. (2007) Mechanical performance evaluation of cast magnesium alloys for automotive and aeronautical applications. J. Eng. Mater. Tech., 129,2422-2430.

[24] Wang, Q., Zhang, Z. M., Zhang, X., and Li, G. J. (2010) New extrusion process of Mg alloy automobile wheels. Trans. Nonfer. Metal. Soc. China, 20(2), 599-603.

[25] Begum, S., Chen, D. L., Xu, S., and Luo, A. A. (2009) Effect of strain ratio and strain rate on low cycle fatigue behavior of AZ31 wrought magnesium alloy. Mater. Sci. Eng. A, 517, 334-343.

[26] Begum, S., Chen, D. L., Xu, S., and Luo, A. A. (2009) Low cycle fatigue properties of an extruded AZ31 magnesium alloy. Int. J. Fatigue, 31, 726-735.

[27] Lin, X. Z. and Chen, D. L. (2008) Strain controlled cyclic deformation behavior of an extruded magnesium alloy. Mater. Sci. Eng. A, 496, 106-113.

[28] Begum, S., Chen, D. L., Xu, S., and Luo, A. A. (2008) Strain-controlled low-cycle fatigue properties of a newly developed extruded magnesium alloy. Metall. Mater. Trans. A, 39, 3014-3026.

[29] Ogarevic, V. V. and Stephens, R. I. (1990) Fatigue of magnesium alloys. Annu. Rev. Mater. Sci., 20, 141-177.

[30] Lorenzo, M., Bravo, P. M., Alegre, J. M., and Cuesta, I. I. (2013) Magnesium alloy defectology AZ91D high-pressure die cast and influence on the fatigue behaviour. Fat. Frac. Eng. Mater. Struct., 36(10), 1017-1026. 
[31] Fouad, Y., Mhaede, M., and Wagner, L. (2011) Effects of mechanical surface treatments on fatigue performance of extruded ZK60 alloy. Fat. Frac. Eng. Mater. Struct., 34(6), 403-407.

[32] Hariharan, K. and Prakash, R. V. (2012) Modification of fatigue strain-life equation for sheet metals considering anisotropy due to crystallographic texture. Fat. Frac. Eng. Mater. Struct., 35(5), 458-465.

[33] Suresh, S. (1998) Fatigue of materials, second ed., Cambridge University Press, Cambridge.

[34] Dieter, G. E. (1986) Mechanical Metallurgy, SI metric ed., McGraw-Hill, Boston, USA.

[35] Fan, C. L., Chen, D. L., and Luo, A. A. (2009) Dependence of the distribution of deformation twins on strain amplitudes in an extruded magnesium alloy after cyclic deformation. Mater. Sci. Eng. A, 519, 38-45.

[36] Sarker, D. and Chen, D. L. (2012) Detwinning and strain hardening of an extruded magnesium alloy during compression. Scr. Mater., 67, 165-168.

[37] Yang, F., Lv, F., Yang, X. M., Li, S. X., Zhang, Z. F., and Wang, Q. D. (2011) Enhanced very high cycle fatigue performance of extruded Mg-12Gd-3Y-0.5Zr magnesium alloy. Mater. Sci. Eng. A, 528, 2231-2238.

[38] Kleiner, S. and Uggowitzer, P. J. (2004) Mechanical anisotropy of extruded Mg-6\%Al1\%Zn alloy. Mater. Sci. Eng. A, 379, 258-263.

[39] Yin, S. M., Yang, H. J., Li, S. X., Wu, S. D., and Yang, F. (2008) Cyclic deformation behavior of as-extruded Mg-3\%Al-1\%Zn. Scr. Mater., 58, 751-754.

[40] Safi-Naqvi, S. H., Hutchinson, W. B., and Barnett, M. R. (2008) Texture and mechanical anisotropy in three extruded magnesium alloys. Mater. Sci. Technol., 24, 1283-1292. 
[41] Ball, E. A. and Prangnell, P. B. (1994) Tensile-compressive yield asymmetries in high strength wrought magnesium alloys. Scr. Metall. Mater., 31, 111-116.

[42] Hirsch, J. and Al-Samman, T. (2013) Superior light metals by texture engineering: optimized aluminum and magnesium alloys for automotive applications. Acta Mater., 61, 818-843.

[43] Hadorn, J. P., Hantzsche, K., Yi, S., Bohlen, J., Letzig, D., and Agnew, S. R. (2012) Effects of solute and second-phase particles on the texture of $\mathrm{Nd}$-containing $\mathrm{Mg}$ alloys. Metall. Mater. Trans. A, 43A(4), 1363-1375.

[44] Li, M. Z., Wang, Y. Q., Li, C., Liu, X. G., and Xu, B. S. (2011) Effects of neodymium rich rare earth elements on microstructure and mechanical properties of as cast AZ31 magnesium alloy. Mater. Sci. Technol., 27, 1138-1142.

[45] Robson, J. D., Twier, A. M., Lorimer, G. W., and Rogers, P. (2011) Effect of extrusion conditions on microstructure, texture, and yield asymmetry in Mg-6Y-7Gd-0.5\%Zr alloy. Mater. Sci. Eng. A, 528, 7247-7256.

[46] Al-Samman, T. (2013) Modification of texture and microstructure of magnesium alloy extrusions by particle-stimulated recrystallization. Mater. Sci. Eng. A, 560, 561-566.

[47] Stanford, N. and Barnett, M.R. (2008) The origin of "Rare Earth" texture development in extruded Mg-based alloys and its effect on tensile ductility. Mater. Sci. Eng. A, 496, 399408 .

[48] Li, X., Al-Samman, T., Mu, S., and Gottstein, G. (2011) Texture and microstructure development during hot deformation of ME20 magnesium alloy: Experiments and simulations. Mater. Sci. Eng. A, 528, 7915-7925. 
[49] Laser, T., Hartig, Ch., Nurnberg, M. R., Letzig, D., and Bormann, R. (2008) The influence of calcium and cerium mischmetal on the microstructural evolution of $\mathrm{Mg}-3 \mathrm{Al}-$ 1Zn during extrusion and resulting mechanical properties. Acta Mater., 56, 2791-2798.

[50] Bohlen, J., Yi, S. B., Letzig, D., and Kainer, K. U. (2010) Effect of rare earth elements on the microstructure and texture development in magnesium-manganese alloys during extrusion. Mater. Sci. Eng. A, 527, 7092-7098.

[51] Cottam, R., Robson, J., Lorimer, G., and Davis, B. (2008) Dynamic recrystallization of Mg and Mg-Y alloys: crystallographic texture development. Mater. Sci. Eng. A, 485, 375382

[52] Li, J., Mishra, R. K., and Sachdev, A. K. (2012) Texture modification during extrusion of some Mg alloys. Metall. Mater. Trans. A, 43A(6), 2148-2157.

[53] Hadorn, J. P., Hantzsche, K., Yi, S. B., Bohlen, J., Letzig, D., Wollmershauser, J. A., and Agnew, S. R. (2012) Role of solute in the texture modification during hot deformation of Mg-rare earth alloys. Metall. Mater. Trans. A, 43A(4), 1347-1362.

[54] Robson, J. D. (2013) Effect of rare-earth additions on the texture of wrought magnesium alloys: the role of grain boundary segregation. Metall. Mater. Trans. A, in press.

[55] Hidalgo-Manrique, P., Yi, S. B., Bohlen, J., Letzig, D., and Pe'rez-Prado, M. T. (2013) Effect of $\mathrm{Nd}$ additions on extrusion texture development and on slip activity in a $\mathrm{Mg}-\mathrm{Mn}$ alloy. Metall. Mater. Trans. A, 44A, 4819-4829.

[56] Hadorn, J. P., Mulay, R. P., Hantzsche, K., Yi, S. B., Bohlen, J., Letzig, D., and Agnew, S. R. (2013) Texture weakening effects in Ce-containing Mg alloys. Metall. Mater. Trans. A, 44A(3), 1566-1576. 
[57] He, S. M., Zeng, X. Q., Peng, L. M., Gao, X., Nie, J. F., and Ding, W. J. (2007) Microstructure and strengthening mechanism of high strength Mg-10Gd-2Y-0.5Zr alloy. $J$ Alloys Comp., 427, 316-323.

[58] Wang, F. H., Dong, J., Jiang, Y. Y., and Ding, W. J. (2013) Cyclic deformation and fatigue of extruded Mg-Gd-Y magnesium alloy. Mater. Sci. Eng. A, 561, 403-410.

[59] Stanford, N., Sha, G., Xia, J. H., Ringer, S. P., and Barnett, M. R. (2011) Solute segregation and texture modification in an extruded magnesium alloy containing gadolinium. Scr. Mater., 65, 919-921.

[60] Stanford, N. and Barnett, M. R. (2008) Effect of composition on the texture and deformation behaviour of wrought Mg alloys. Scr. Mater., 58, 179-182.

[61] Stanford, N., Atwell, D., Beer, A., Davies, C. H., and Barnett, M. R. (2008) Effect of microalloying with rare-earth elements on the texture of extruded magnesium-based alloys. Scr. Mater., 59, 772-775.

[62] Unsworth, W. (1989) The role of rare earth elements in the development of magnesium base alloys. Int. J. Mater. Prod. Technol., 4(4), 359-378.

[63] Eliezer, D., Aghion, E., and Froes, F. H. (1998) Magnesium science, technology and applications. Adv. Perfor. Mater., 5, 201-212.

[64] Agnew, W. G. (1974) Reducing automotive emissions. Science, 183(4122), 254-256.

[65] Ke, W., Han, E. H., Han, Y. F., Kainer K., and Luo, A. A. (2005) Mechanical properties and creep behavior of Mg-Al-Ca alloys. Proceedings of International Conference on Magnesium - Science, Technology and Applications. Beijing, China. Mater. Sci. Forum., 488-489. 
[66] Friedrich, H. E. and Mordike, B. L. (2006) Magnesium Technology-Metallurgy, Design Data, Applications, Springer-Verlag Berlin Heidelberg, Germany.

[67] Blawert, C., Hort, N., and Kainer, K. U. (2004) Automotive applications of magnesium and its alloys. Trans. Indian Inst. Met., 57(4), 397-408.

[68] United States Automotive Materials Partnership (USAMP). 2006. Magnesium vision 2020: A North American automotive strategic vision for magnesium.

[69] Hort, N., Huang, Y., Fechner, D., Stormer, M., Blawert, C., Witte, F., Vogt, C., Drucker, H., Willumeit, R., Kainer, K. U., and Feyerabend, F. (2010) Magnesium alloys as implant materials-Principles of property design for Mg-RE alloys. Acta Biomater., 6, 1714-1725.

[70] Peng, Q., Wang, J., Wu, Y., and Wang, L. (2006) Microstructures and tensile properties of Mg-8Gd-0.6Zr-xNd-yA (x+y=3, mass\%) alloys. Mater. Sci. Eng. A, 433, 133-138.

[71] Apps, P. J., Karimzadeh, H., King, J. F., and Lorimer, G. W. (2003) Phase composition in magnesium-rare earth alloys containing yttrium, gadolinium or dysprosium. Scr. Mater., 48, $475-481$.

[72] Apps, P. J., Karimzadeh, H., King, J. F., and Lorimer, G. W. (2003) Precipitation reactions in magnesium-rare earth alloys containing yttrium, gadolinium or dysprosium. Scr. Mater., 48, 1023-1028.

[73] Chang, J., Guo, X., He, S., Fu, P., Peng, L., and Ding, W. (2008) Investigation of the corrosion for Mg-xGd-3Y-0.4Zr ( $\mathrm{x}=6,8,10,12$ wt.\%) alloys in a peak-aged condition. Corrosion, 50, 166-177.

[74] Cizek, J., Prochazka, I., Smola, B., Stulikova, I., and Ocenasek, V. (2007) Influence of deformation on precipitation process in Mg-15 wt.\% Gd alloy. J. Alloys Comp., 430, 9296. 
[75] Gao, L., Chen, R. S., and Han, E. H. (2009) Effects of rare-earth elements Gd and Y on the solid solution strengthening of Mg alloys. J. Alloys Comp., 481, 379-384.

[76] Gao, X., He, S. M., Zeng, X. Q., Teng, L. M., Ding, W. J., and Nie, J. F. (2006) Microstructure evolution in a $\mathrm{Mg}-15 \mathrm{Gd}-0.5 \mathrm{Zr}$ (wt.\%) alloy during isothermal aging at 250 ${ }^{\circ}$ C. Mater. Sci. Eng. A, 431, 322-327.

[77] Gao, Y., Wang, Q., Gu, J., Zhao, Y., and Tong, Y. (2007) Behavior of Mg-15Gd-5Y-0.5Zr alloy during solution heat treatment from 500 to $540^{\circ}$ C. Mater. Sci. Eng. A, 45, 117-123.

[78] Liu, K., Zhang, J., Rokhlin, L. L., Elkin, F. M., Tang, D., and Meng, J. (2009) Microstructures and mechanical properties of extruded Mg-8Gd- $0.4 \mathrm{Zr}$ alloys containing Zn. Mater. Sci. Eng. A, 505, 13-19.

[79] Peng, Q., Wang, L., Wu, Y., and Wang, L. (2009) Structure stability and strengthening mechanism of die-cast Mg-Gd-Dy based alloy. J. Alloys Comp., 469, 587-592.

[80] Peng, Q., Hou, X., Wang, L., Wu, Y., Cao, Z., and Wang, L. (2009) Microstructure and mechanical properties of high performance Mg-Gd based alloys. Mater. Des., 30, 292-296.

[81] Peng, Q., Dong, H., Wang, L., Wu, Y., and Wang, L. (2008) Aging behavior and mechanical properties of Mg-Gd-Ho alloys. Mater. Charact., 59, 983-986.

[82] Peng, Q., Dong, H., Wu, Y., and Wang, L. (2008) Age hardening and mechanical properties of Mg-Gd-Er alloy. J. Alloys Comp., 456, 395-399.

[83] Peng, Q., Wu, Y., Fang, D., Meng, J., and Wang, L. (2006) Microstructures and properties of melt-spun and as-cast Mg-20Gd binary alloy. J. Rare Earths, 24, 466-470.

[84] Rokhlin, L. L. (2003) Magnesium alloys containing rare earth elements-structure and properties. Taylor \& Francis, London. 
[85] Yang, Z., Li, J. P., Zhang, J. X., Lorimer, G. W., and Robson, J. (2008) Review on research and development of magnesium alloys. Acta Metall. Sin. (Engl. Lett.), 21(5), 313328.

[86] Yang, Y., Liu, Y. B., Qin, S. Y., and Fang, Y. (2006) High cycle fatigue properties of diecast magnesium alloy AZ91D addition of different concentrations of cerium. J. Rare Earths, 24, 591-595.

[87] Liu, X. B., Chen, R. S., and Han, E. H. (2008) Effects of ageing treatment on microstructures and properties of $\mathrm{Mg}-\mathrm{Gd}-\mathrm{Y}-\mathrm{Zr}$ alloys with and without $\mathrm{Zn}$ additions. $J$. Alloys Comp., 465, 232-238.

[88] Xu, Y. L., Zhang, K., Li, X. G., Lei, J., Yang, Y. S., and Luo, T. J. (2008) High cycle fatigue properties of die-cast magnesium alloy AZ91D-1\%MM. Trans. Nonferr. Met. Soc. China, 18, 306-311.

[89] Yang, Y. and Liu, Y. B. (2008) High cycle fatigue characterization of two die-cast magnesium alloys. Mater. Charact., 59, 567-570.

[90] Yang, Y. and Li, X. S. (2010) Influence of neodymium on high cycle fatigue behavior of die cast AZ91D magnesium alloy. J. Rare Earths, 28(3), 456-460.

[91] Mokhtarishirazabad, M., Boutorabi, S. M. A., Azadi, M., and Nikravan, M. (2013) Effect of rare earth elements on high cycle fatigue behavior of AZ91 alloy. Mater. Sci. Eng. A, 587, 179-184.

[92] Bayani, H. and Saebnoori, E. (2009) Effect of rare earth elements addition on thermal fatigue behaviors of AZ31 magnesium alloy J. Rare Earths, 27(2), 255-258. 
[93] Honma, T., Ohkubo, T., Hono, K., and Kamado, S. (2005) Chemistry of nanoscale precipitates in $\mathrm{Mg}-2.1 \mathrm{Gd}-0.6 \mathrm{Y}-0.2 \mathrm{Zr}$ (at.\%) alloy investigated by the atom probe technique. Mater. Sci. Eng. A, 395, 301-306.

[94] Gorsse, S., Hutchinson, C. R., Chevalier, B., and Nie, J. F. (2005) A thermodynamic assessment of the $\mathrm{Mg}-\mathrm{Nd}$ binary system using random solution and associate models for the liquid phase. J. Alloys Comp., 39, 253-262.

[95] Peng, Z. -K., Zhang, X. -M., Chen, J. -M., Xiao, Y., and Jiang, H. (2005) Grain refining mechanism in Mg-9Gd-4Y alloys by zirconium. Mater. Sci. Technol., 21, 722-726.

[96] Chen, L., Wang, C., Wu, W., Liu, Z., Stoica, G. M., Wu, L., and Liaw, P. K. (2007) Lowcycle fatigue behavior of an as-extruded AM50 magnesium alloy. Metall. Mater. Trans. A, 38, 2235-2241.

[97] Lin X. Z. and Chen, D. L. (2008) Strain hardening and strain-rate sensitivity of an extruded magnesium alloy. J. Mater. Eng. Perform., 17(6), 894-901.

[98] Fairman, M., Afrin, N., Chen, D. L., Cao, X. J., and Jahazi, M. (2007) Microstructural evaluation of friction stir processed AZ31B-H24 magnesium alloy. Canadian Metall. Quarterly, 46(4), 425-432.

[99] Afrin, N., Chen, D. L., Cao, X., and Jahazi, M. (2007) Strain hardening behavior of a friction stir welded magnesium alloy. Scr. Mater., 57(11), 1004-1007.

[100] Afrin, N., Chen, D. L., Cao, X., and Jahazi, M. (2008) Microstructure and tensile properties of friction stir welded AZ31B magnesium alloy. Mater. Sci. Eng. A, 472(1-2), $179-186$. 
[101] Bettles, C. J. and Gibson, M. A. (2003) Microstructural design for enhanced elevated temperature properties in sand-castable magnesium alloys. Adv. Eng. Mater., 12, pp. 859865.

[102] Dong, J., Liu, W. C., Song, X., Zhang, P., Ding, W. J., and Korsunsky, A.M. (2010) Influence of heat treatment on fatigue behavior of high-strength Mg-10Gd-3Y alloy. Mater. Sci. Eng. A, 725, 6053-6063.

[103] Liu, W. C., Dong, J., Song, X., Belnoue, J. P., Hofmann, F., Ding, W. J., and Korsunsky, A. M. (2011) Effect of microstructures and texture development on tensile properties of Mg-10Gd-3Y alloy. Mater. Sci. Eng. A, 528, 2250-2258.

[104] He, S. M., Zeng, X. Q., Peng, L. M., Gao, X., Nie, J. F., and Ding, W. J. (2006) Precipitation in a Mg-10Gd-3Y-0.4Zr (wt.\%) alloy during isothermal ageing at $250^{\circ} \mathrm{C}$. $J$. Alloys Comp., 421, 309-313.

[105] Nie, J. F. (2012) Precipitation and hardening in magnesium alloys. Metall. Mater. Trans. A, 43A, 3891-3939.

[106] Kawabata, T., Fukuda, Y., Matsuda, K., Kamado, S., Kojima, Y., and Ikeno, S. (2007) HRTEM Observation of the precipitates in heat-resistant Mg-Gd-Y-Zr alloy. Mater. Sci. Forum, 539-543, 1769-1774.

[107] Liu, Z. J., Wu, G. H., Liu, W. C., Pang, S., and Ding, W. J. (2012) Effect of heat treatment on microstructures and mechanical properties of sand-cast Mg-4Y-2Nd-1Gd-0.4Zr magnesium alloy. Trans. Nonferr. Met. Soc. China, 22, 1540-1548.

[108] Sarker, D. and Chen, D. L. (2013) Texture transformation in an extruded magnesium alloy under pressure. Mater. Sci. Eng. A, 582, 63-67. 
[109] Senn, J. W. and Agnew, S. R. Proc. Magnesium Technology in the Global Age, Pekguleryuz, M. O. and Mackenzie, L. W. F. eds., Montreal, PQ, Canada, 2006, pp. 115130.

[110] Senn, J. W. and Agnew, S. R. Magnesium Technology 2008: Proc. TMS, M.O. Pekguleryuz, N.R. Neelameggham, R.S. Beals, and E.A. Nyberg, eds., TMS, Warrendale, PA, 153-158.

[111] Liang, S. Q., Guan, D. K., Chen, L., Gao, Z. H., Tang, H. X., and Tong, X. T. (2011) Precipitation and its effect on age-hardening behavior of as-cast Mg-Gd-Y alloy. Mater. Des., 32, 361-364.

[112] Wang, Q., Wu, G., Zheng, H. Q., Chen, B., Zheng, Y., and Ding, W. (2010) A comparative study of Mg-Gd-Y-Zr alloy cast by metal mould and sand. China Found. J., 7, $6-12$.

[113] Zhang, F., Zhang, K. X., Tan, C. W., Yu, X. D., Ma, H. L., Wang, F. C., and Cai, H. N. (2011) Microstructure and mechanical properties of Mg-Gd-Y-Zr alloys processed by equal channel angular pressing. Trans. Nonferr. Met. Soc. China, 21, 2140-2146.

[114] Zhu, R., Wu, Y. J., Wang, J. T., and Lu, K. C. (2011) Mechanical anisotropy of extruded Mg-10Gd-2Y-0.5Zr alloy. Adv. Mater. Res., 320, 222-227.

[115] Stanford, N., Atwell, D., and Barnett, M. R. (2010) The effect of Gd on the recrystallisation, texture and deformation behaviour of magnesium-based alloys. Acta Mater., 58, 6773-6783.

[116] Stanford, N. (2010) Micro-alloying Mg with Y, Ce, Gd and La for texture modification-a comparative study. Mater. Sci. Eng. A, 527, 2669-2677. 
[117] Bohlen, J., Nurnberg, M. R., Senn, J. W., Letzig, D., and Agnew, S. R. (2007) The texture and anisotropy of magnesium-zinc-rare earth alloy sheets. Acta Mater., 55, 2101-2112.

[118] Antion, C., Donnadieu, P., Perrard, F., Deschamps, A., Tassin, C., and Pisch, A. (2003) Hardening precipitation in a Mg-4Y-3RE alloy. Acta Mater., 51, 5335-5348.

[119] Nie, J. F. and Muddle, B. C. (2000) Characterisation of strengthening precipitate phases in a Mg-Y-Nd alloy. Acta Mater., 48, 1691-1703.

[120] Hantzsche, K., Bohlen, J., Wendt, J., Kainer, K. U., Yi, S. B., and Letzig, D. (2010) Effect of rare earth additions on microstructure and texture development of magnesium alloy sheets. Scr. Mater., 63, 725-730.

[121] Nie, J. F., Gao, X., and Zhu, S.M. (2005) Enhanced age hardening response and creep resistance of Mg-Gd alloys containing Zn. Scr. Mater., 53, 1049-1053.

[122] Agnew, S. R., Yoo, M. H., and Tome, C.N. (2001) Application of texture simulation to understanding mechanical behavior of $\mathrm{Mg}$ and solid solution alloys containing $\mathrm{Li}$ or Y. Acta Mater., 49, 4277-4289.

[123] Yi, S., Bohlen, J., Heinemann, F., and Letzig, D. (2010) Mechanical anisotropy and deep drawing behaviour of AZ31 and ZE10 magnesium alloy sheets. Acta Mater., 58, 592-605.

[124] Brown, D. W., Jain, A., Agnew, S. R., and Clausen, B. (2007) Twinning and detwinning during cyclic deformation of Mg alloy AZ31B. Mater. Sci. Forum, 539-543, 3407-3413.

[125] Noster, U. and Scholtes, B. (2003) Isothermal strain-controlled quasi-static and cyclic deformation behavior of magnesium wrought alloy AZ31. Mater. Res. Adv. Tech. (Zeitschrift fuer Metallkunde), 94, 559-563.

[126] Hasegawa, S., Tsuchida, Y., Yano, H., and Matsui, M. (2007) Evaluation of low cycle fatigue life in AZ31 magnesium alloy. Int. J. Fatigue, 29, 1839-1845. 
[127] Chamos, A. N., Pantelakis, Sp. G., Haidemenopoulos, G. N., and Kamoutsi, E. (2008) Tensile and fatigue behaviour of wrought magnesium alloys AZ31 and AZ61. Fat. Frac. Eng. Mater. Struct., 31(9), 812-821.

[128] Chamos, A. N., Charitidis, C. A., Skarmoutsou, A., and Pantelakis, Sp. G. (2010) An investigation on the high stress sensitivity of fatigue life of rolled AZ31 magnesium alloy under constant amplitude fatigue loading. Fat. Frac. Eng. Mater. Struct., 33(4), 252-265.

[129] Wu, L., Jain, A., Brown, D. W., Stoica, G. M., Agnew, S. R., Clausen, B., Fielden, D. E., and Liaw, P. K. (2008) Twinning-detwinning behavior during the strain-controlled lowcycle fatigue testing of a wrought magnesium alloy, ZK60A. Acta Mater., 56, 688-695.

[130] Wu, L., Agnew, S. R., Brown. D. W., Stoica, G. M., Clausen, B., Jain, A., Fielden, D. E., and Liaw, P. K. (2008) Internal stress relaxation and load redistribution during the twinning-detwinning-dominated cyclic deformation of a wrought magnesium alloy, ZK60A. Acta Mater., 56, 3699-3707.

[131] Eisenmeier, G., Holzwarth, B., Hoppel, H. W., and Mughrabi, H. (2001) Cyclic deformation and fatigue behaviour of the magnesium alloy AZ91. Mater. Sci. Eng. A, 319321, 578-582.

[132] Chen, L., Shen, J., Wu, W., Li, F., Wang, Y., and Liu, Z. (2005) Low-cycle fatigue behavior of magnesium alloy AZ91. Mater. Sci. Forum, 488-489, 725-728.

[133] Horstemeyer, M. F., Yang, N., Gall, K., McDowell, D. L., Fan, J., and Gullett, P. M. (2004) High cycle fatigue of a die cast AZ91E-T4 magnesium alloy. Acta Mater., 52, $1327-1336$. 
[134] Liu, Z., Xu, Y. Y., Wang, Z. G., Wang, Y., and Liu, Z. Y. (2000) Low cycle fatigue behavior of AZ91HP alloy in as high pressure die casting. Acta Metallurgica Sinica (English Letters), 13, 961-966.

[135] Islamgaliev, R. K., Kulyasova, O. B., Mingler, B., Zehetbauer, M., and Minkow, A. (2008) Structure and fatigue properties of the Mg alloy AM60 processed by ECAP. Mater. Sci. Forum, 584-586, 803-808.

[136] Kulyasova, O. B., Islamgaliev, R., Mingler, B., and Zehetbauer, M. (2009) Microstructure and fatigue properties of the ultrafine-grained AM60 magnesium alloy processed by equal-channel angular pressing. Mater. Sci. Eng. A, 503, 176-180.

[137] El Kadiri, H., Horstemeyer, M. F., Jordon, J. B., and Xue, Y. (2008) Fatigue crack growth mechanisms in high-pressure die-cast magnesium alloys. Metall. Mat. Trans. A, 39, 190205.

[138] Horstemeyer, M. F., Yang, N., Gall, K., McDowell, D., Fan, J., and Gullett, P. (2002) High cycle fatigue mechanisms in a cast AM60B magnesium alloy. Fat. Frac. Eng. Mater. Struct., 25, 1045-1056.

[139] Liu, Z., Ji, H., Lin, L, Chen, L., Wu, W., and Yang, L. (2007) Cyclic deformation behavior and potential automobile application of magnesium die casting alloys AZ91 and AM50. Mater. Sci. Forum, 539-543, 1626-1631.

[140] El Kadiri, H., Xue, Y., Horstemeyer, M. F., Jordon, J. B., and Wang, P. T. (2006) Identification and modeling of fatigue crack growth mechanisms in a die-cast AM50 magnesium alloy. Acta Mater., 54, 5061-5076.

[141] Liu, Z., Wang, Z. G., Wang, Y., and Liu, Z. Y. (1999) Cyclic deformation behavior of high pressure die casting alloy AM50. J. Mater. Sci. Lett., 18, 1567-1569. 
[142] Yang, Y. and Liu, Y. B. (2007) The effect of cerium on high-cycle fatigue properties of die-cast magnesium alloy. Fat. Frac. Eng. Mater. Struct., 30(12), 1149-1157.

[143] Nascimento, L., Yi, S., Bohlen, J., Fuskova, L., Letzig, D., and Kainer, K. U. (2010) High cycle fatigue behaviour of magnesium alloys. Procedia Eng., 2, 743-750.

[144] Yang, X. M., Yang, H. J., Yang, F., Yin, S. M., Wang, W., Li, S. X., and Wang, Q. D. (2009) Tensile and isothermal fatigue behaviors of Mg-12Gd-3Y-0.5Zr alloy at high temperature. J. Mater. Sci. Technol., 25(6), 731-737.

[145] Song, X., Liu, W. C., Belnoue, J. P., Dong, J., Wu, G. H., Ding, W. J, Kimber, S. A. J., Buslaps, T., Lunt, A. J. G., and Korsunsky, A.M. (2012) An eigenstrain-based finite element model and the evolution of shot peening residual stresses during fatigue of GW103 magnesium alloy. Int. J. Fatigue, 42, 284-295.

[146] Fu, Q. Q., Li, Y. S., Liu, G. W., and Li, H. (2012) Low cycle fatigue behavior of AZ91D magnesium alloy containing rare-earth Ce element. Procedia Eng., 27, 1794-1800.

[147] Wu, L., Yang, Z., Xia, W., Chen, Z., and Yang, L. (2012) The cyclic softening and evolution of microstructures for Mg-10Gd-2.0Y-0.46Zr alloy under low cycle fatigue at 573 K. Mater. Des., 36, 47-53.

[148] Yin, S. M. and Li, S. X. (2013) Low-cycle fatigue behaviors of an as-extruded Mg12\%Gd-3\%Y-0.5\%Zr alloy. J. Mater. Sci. Technol., 29(8), 775-780.

[149] Zhu, R., Cai, X. T., Wu, Y. J., Liu, L. G., Ji, W. Q., and Hua, B. (2014) Low-cycle fatigue behavior of extruded Mg-10Gd-2Y-0.5Zr alloys. Mater. Des., 53, 992-997.

[150] Potzies, C. and Kainer, K. U. (2004) Fatigue of magnesium alloys. Adv. Eng. Mater, 6(5), 281-289. 
[151] Yang, F., Yin, S. M., Li, S. X., and Zhang, Z. F. (2008) Crack initiation mechanism of extruded AZ31 magnesium alloy in the very high cycle fatigue regime. Mater. Sci. Eng. A, 491, 131-136.

[152] Patel, H. A., Chen, D. L., Bhole, S. D., and Sadayappan, K. (2013) Low cycle fatigue behavior of a semi-solid processed AM60B magnesium alloy. Mater. Des., 49, 456-464.

[153] Patel, H. A., Rashidi, N., Chen, D. L., Bhole, S. D., and Luo, A. A. (2012) Cyclic deformation behavior of a super-vacuum die cast magnesium alloy. Mater. Sci. Eng. A, 546, 72-81.

[154] Goodenberger, D. L. and Stephens, R. I. (1993) Fatigue of AZ91E-T6 Cast Magnesium Alloy. J. Eng. Mater. Tech., 115, 391-397.

[155] Patel, H. A., Chen, D. L., Bhole, S. D., and Sadayappan, K. (2010) Cyclic deformation and twinning in a semi-solid processed AZ91D magnesium alloy. Mater. Sci. Eng. A, 528, 208-219.

[156] Jordon, J. B., Gibson, J. B., Horstemeyer, M. F., El Kadiri, H., Barid, J. C., and Luo, A. A. (2011) Effect of twinning, slip, and inclusions on the fatigue anisotropy of extrusiontextured AZ61 magnesium alloy. Mater. Sci. Eng. A, 528, 6860-6871.

[157] Lv, F., Yang, F., Duan, Q. Q., Yang, Y. S., Wu, S. D., Li, S. X., and Zhang, Z. F. (2011) Fatigue properties of rolled magnesium alloy (AZ31) sheet: Influence of specimen orientation. Int. J. Fatigue, 33, 672-682.

[158] Yu, Q., Zhang, J., Jiang, Y., and Li, Q. (2012) An experimental study on cyclic deformation and fatigue of extruded ZK60 magnesium alloy. Int. J. Fatigue, 36, 47-58. 
[159] Luo, T. J., Yang, Y. S., Tong, W. H., Duan, Q. Q., and Dong, X. G. (2010) Fatigue deformation characteristic of as-extruded AM30 magnesium alloy. Mater. Des., 31, 16171621.

[160] Lee, S. G., Patel, G. R., and Gokhale, A. M. (2005). Inverse surface macro-segregation in high-pressure die-cast AM60 magnesium alloy and its effects on fatigue behavior. Scr. Mater., 52, 1063-1068.

[161] Vinogradov, A., Washikita, A., Kitagawa, K., and Kopylov, V. I. (2003) Fatigue life of fine-grain Al-Mg-Sc alloys produced by equal-channel angular pressing. Mater. Sci. Eng. A, 349, 318-326.

[162] Xiong, Y., Yu, Q., and Jiang, Y. (2012) Multiaxial fatigue of extruded AZ31B magnesium alloy. Mater. Sci. Eng. A, 546, 119-128.

[163] Bachmann, F., Hielscher, R., and Schaeben, H. (2010) Texture analysis with MTEX free and open source software toolbox. Solid State Phenom., 160, 63-68.

[164] Standard test methods of tension testing of metallic materials, ASTM E8 / E8M-13a.

[165] Standard test methods of compression testing of metallic materials at room temperature, ASTM E9-09.

[166] Standard test method for determining volume fraction by systematic manual point count, ASTM E562-11.

[167] Standard test method for strain-controlled fatigue testing, ASTM E606 / E606M-12.

[168] Li, W. P., Zhou, H., and Li, Z. F. (2009) Effect of gadolinium on microstructure and rolling capability of AZ31 alloy. J. Alloys Comp., 475(1-2), 227-232. 
[169] Fang, X. Y., Yi, D. Q., Wang, B., Luo, W. H., and Gu, W. (2006) Effect of yttrium on microstructures and mechanical properties of hot rolled AZ61 wrought magnesium alloy. Trans. Nonfer. Met. Soc. China, 16(5), 1053-1058.

[170] Yu, K., Rui, S. T., Song, J. M., Li, W. X., and Guo, L. (2008) Effects of grain refinement on mechanical properties and microstructures of AZ31 alloy. Trans. Nonfer. Met. Soc. China, 18, s39-s43.

[171] Jia, S. G., Liu, P., Dong, Q. M., Xia, C. Q., Tian, B. H., and Ren, F. Z. (2010) Effect of rare earth neodymium on microstructure and properties of AZ31B magnesium alloy. Trans. Mater. Heat. Treat., 31(7), 99-103.

[172] Zhang, J., Zhang, X. F., Pan, F. S., Liu, C. P., and He, Q. B. (2009) Microstructure and mechanical properties of an $\mathrm{Mg}-\mathrm{Zn}-\mathrm{Zr}$ alloy modified by rare earth of Erbium. Mater. Sci. Forum, 610-613, 810-814.

[173] Humphreys, F. J. (1997) Unified theory of recovery, recrystallization and grain growth, based on the stability and growth of cellular microstructures - II. The effect of secondphase particles. Acta. Mater., 45(12), 5031-5039.

[174] Xin, R. L., Song, B., Zeng, K., Huang, G. J., and Liu, Q. (2012) Effect of aging precipitation on mechanical anisotropy of an extruded Mg-Y-Nd alloy. Mater. Des., 34, 384-388.

[175] Chen, X. H., Pan, F. S., Mao, J. J., Wang, J. F., Zhang, D. F., Tang, A. T., and Peng, J. (2011) Effect of heat treatment on strain hardening of ZK60 Mg alloy. Mater. Des., 32, $1526-1530$.

[176] Rollett, A. D., Kocks, U. F., and Doherty, R. D. (1987) Stage IV work hardening in cubic metals. Symp on Formability Metall Struct (The Metallurgical Society, AIME), 211-225. 
[177] Haasen, P. (1989) A cell theory for stage IV work hardening of metals and semiconductors. J. Phys. France, 50, 2445-2454.

[178] Agnew, S.R. (2002) Plastic anisotropy of magnesium alloy AZ31B sheet. In: Kaplan, H. I. (Ed.), TMS Annual Meeting, Magnesium Technology 2002. TMS, Seattle, Washington, USA, pp. 169-174.

[179] Partridge, P. G. (1967) Crystallography and deformation modes of hexagonal close packed metals. Met. Rev., 12, 169-194.

[180] Koike, J., Kobayashi, T., Mukai, T., Watanabe, H., Suzuki, M., Maruyama, K., and Higashi, K. (2003) The activity of non-basal slip systems and dynamic recovery at room temperature in fine-grained AZ31B magnesium alloys. Acta. Mater., 51, 2055-2065.

[181] Dobron, P., Chmelik, F., Yi, S., Parfenenko, K., Letzig, D., and Bohlen, J. (2011) Grain size effects on deformation twinning in an extruded magnesium alloy tested in compression. Scr. Mater., 65, 424-427.

[182] Yin, S. M., Wang, C. H., Diao, Y. D., Wu, S. D., and Li, S. X. (2011) Influence of grain size and texture on the yield asymmetry of Mg-3Al-1Zn Alloy. J. Mater. Sci. Tech., 27(1), $29-34$.

[183] Stanford, N. and Barnett, M. R. (2009) Effect of particles on the formation of deformation twins in a magnesium-based alloy. Mater. Sci. Eng. A, 516, 226-234.

[184] Reed-Hill R. E. and Robertson, W. D. (1957) Additional modes of deformation twinning in magnesium. Acta Metall., 5, 717-727.

[185] Yoshinaga, H. and Horiuchi, R. (1963) On flow stress of $\alpha$-solid solution Mg-Li alloy single crystals. Trans. JIM, 4, 1. 
[186] Wonsiewicz, B. C. and Backofen, W. A. (1967) Plasticity of magnesium crystals. Trans. TMS-AIME, 239, 1422.

[187] Barnett, M. R., Keshavarz, Z., Beer, A. G., and Atwell, D. (2004) Influence of grain size on the compressive deformation of wrought Mg-3Al-1Zn. Acta Metall., 52 (17), 5093-5103.

[188] Schmid, E. (1931) Beitrage zur Physik und Metallographie des Magnesiums. Z. Elektrochem, 37, 447.

[189] Gharghouri, M. A., Weatherly, G. C., Embury, J. D., and Root, J. (1999) Study of the mechanical properties of Mg-7.7at.\% Al by in-situ neutron diffraction. Philos. Mag. A, 79 (7), 1671-1695.

[190] Yakubtsov, I. A., Diak, B. J., Sager, C. A., Bhattacharya, B., MacDonald, W. D., and Niewczas, M. (2008) Effects of heat treatment on microstructure and tensile deformation of Mg AZ80 alloy at room temperature. Mater. Sci. Eng. A, 496, 247-255.

[191] Kocks, U. F. and Mecking, H. (2003) Physics and phenomenology of strain hardening: The FCC case. Prog. Mater. Sci., 48(3), 171-273.

[192] Argon, A. S. and Haasen, P. (1993) A new mechanism of work hardening in the late stages of large strain plastic flow in f.c.c and diamond cubic crystals. Acta. Metall. Mater., 41(1), 3289-3306.

[193] Nix, W., Gibeling, J., and Hughes, D. (1985) Time-dependent deformation of metals. Met. Trans., 16A, 2215-2226.

[194] Slooff, F. A., Zhou, J., Duszczyk, J., and Katgerman, L. (2007) Constitutive analysis of wrought magnesium alloy Mg-Al4-Zn1. Scr. Mater., 57(8), 759-762. 
[195] Wright, R. N. and Paulson, M. S. (1998) Constitutive equation development for high strain deformation processing of aluminum alloys. J. Mater. Process. Technol., 80-81, 556559.

[196] Hou, Q. Y. and Wang, J. T. (2010) A modified Johnson-Cook constitutive model for MgGd-Y alloy extended to a wide range of temperatures. Comput. Mater. Sci., 50, 147-152.

[197] Lin, Y. C. and Chen, X. M. (2011) A critical review of experimental results and constitutive descriptions for metals and alloys in hot working. Mater. Des., 32, 1733-1759.

[198] Bodner, S. R. and Partom, Y. (1975) Constitutive equation, for elastic-viscoplastic strain hardening materials. J. Appl. Mech., 42, 385-389.

[199] Johnson, G. R. and Cook, W. H. (1983) A constitutive model and data for metals subjected to large strains, high strain rates and high temperatures. Proceedings of the 7th International Symposium on Ballistics, Netherlands.

[200] Zerilli, F. J. and Armstrong, R. W. (1987) Dislocation-mechanics based constitutive relations for material dynamics calculations. J. Appl. Phys., 61(5), 1816.

[201] Johnson, G. R. and Cook, W. H. (1985) Fracture characteristics of three metals subjected to various strains, strain rates, temperatures and pressures. Eng. Fract. Mech., 21(1), 31-48.

[202] Lin, Y. C. and Liu, Ge. (2010) A new mathematical model for predicting flow stress of typical high-strength alloy steel at elevated high temperature. Comput. Mater. Sci., 48, 5458.

[203] Meyers, M. A., Chen, Y. -J., Marquis, F. D. S., and Kim, D. S. (1995) High-strain, highstrain-rate behavior of tantalum. Metall. Mater. Trans. A, 26, 2493-2501.

[204] Wang, Y., Zhou, Y. X., and Xia, Y. M. (2004) A constitutive description of tensile behavior for brass over a wide range of strain rates. Mater. Sci. Eng. A, 372, 186-190. 
[205] Liu, Z., Mao, P., and Wang, C. (2011) High strain rate compression behavior and constitutive relation of as-extruded Mg-Gd-Y magnesium alloy. Mater. Sci. Forum, 686, $162-167$.

[206] Sivakesavam, O., Rao, I. S., and Prasad, Y. V. (1993) Processing map for hot working of as cast magnesium. Mater. Sci. Technol., 9, 805-810.

[207] Mwembela, A., Konopleva, E. B., and McQueen, H. J. (1997) Microstructural development in Mg alloy AZ31 during hot working. Scr. Mater., 37(11), 1789-1795.

[208] Lin, Y. C., Li, L. T., and Jiang, Y. Q. (2013) Hot deformation and processing map of a typical Al-Zn-Mg-Cu alloy. J. Alloys Comp., 550, 438-445.

[209] Lin, Y. C., Chen, X. M., and Liu, G. (2010) A modified Johnson-Cook model for tensile behaviors of typical high-strength alloy steel. Mater. Sci. Eng. A, 527, 6980-6986.

[210] Hollomon, J. H. (1945) Tensile deformation. Trans. AIME, 162, 268-290.

[211] Ludwik, P. (1909) Elemente der Technologischen Mechanik. Springer-Verlag, Berlin.

[212] Liu, W., Wu, G., Zhai, C., Ding, W., and Korsunsky, A. M. (2013) Grain refinement and fatigue strengthening mechanisms in as-extruded $\mathrm{Mg}-6 \mathrm{Zn}-0.5 \mathrm{Zr}$ and $\mathrm{Mg}-10 \mathrm{Gd}-3 \mathrm{Y}-0.5 \mathrm{Zr}$ magnesium alloys by shot peening. Int. J. Plast., 49, 16-35.

[213] Wang, L., Qin, Q. Y., Tan, C. W., Zhang, F., and Li, S. K. (2011) Effect of aging treatment on dynamic behavior of Mg-Gd-Y alloy. Conf. Proc. Soc. Exp. Mech. Series 1, 311-317.

[214] Okamoto, H. (1993) Gd-Mg (Gadolinium-Magnesium). J. Phase Equilib., 14(4), 534-535.

[215] Okamoto, H. (2010) Mg-Y (Magnesium-Yttrium). J. Phase Equilib. Diffus., 31(2), 199.

[216] Arroyave, R., Shin, D., and Liu, Z. K. (2005) Modification of the thermodynamic model for the Mg-Zr system. Comput. Coupling Phase Diagrams Thermochem., 29, 230-238. 
[217] Wang, Z. H., Du, W. B., Wang, X. D., Liu, K., and Li, S. B. (2013) Microstructure evolution of Mg-9Gd-2Er-0.4Zr alloy during solid solution treatment. Trans. Nonferr. Met. Soc. China, 23(3), 593-598.

[218] Gao, L., Zhou, J., Sun, Z. M., Chen, R. S., and Han, E. H. (2011) First-principles calculations of the $\beta^{\prime}-\mathrm{Mg}_{7} \mathrm{Gd}$ precipitate in $\mathrm{Mg}-\mathrm{Gd}$ binary alloys. Chin. Sci. Bull., 56(11), $1142-1146$.

[219] Nishijima, M., Hiraga, K., Yamasaki, M., and Kawamura, Y. (2006) Characterization of $\beta^{\prime}$ phase precipitates in an Mg-5at\%Gd alloy aged in a peak hardness condition, studied by high-angle annular detector dark-field scanning transmission electron microscopy. Mater. Trans., 47(8), 2109-2112.

[220] Mackenzie, L. W. F. and Pekguleryuz, M. O. (2008) The recrystallization and texture of magnesium-zinc-cerium alloys. Scr. Mater., 59, 665-668.

[221] Perez-Prado, M. T. and Ruano, O. A. (2002) Texture evolution during annealing of magnesium AZ31 alloy. Scr. Mater., 46, 149-155.

[222] Yang, W. G. and Koo, C. H. (2003) Capacity for deformation and the evaluation of flow stress of hot extruded Mg-8Al-xRE alloys at elevated temperatures. Mater. Trans., 44, 1198-1203.

[223] Wang, S. Q., Liu, J. H., and Chen, D. L. (2013) Strain-controlled fatigue properties of dissimilar welded joints between Ti-6Al-4V and Ti17 alloys. Mater. Des., 49, 716-727.

[224] Laird, C. (1967) Fatigue Crack Propagation, ASTM STP 415, ASTM International, West Conshohocken: PA.

[225] Beaber, A. and Gerberich, W. (2010) Alloys: strength from modelling. Nature Mater., 9, 698-699. 
[226] Matsuda, M., Ando, S., and Nishida, M. (2005) Dislocation structure in rapidly solidified Mg97Zn 1Y2 alloy with long period stacking order phase Mater. Trans., 46, 361-364.

[227] Yin, D. D., Wang, Q. D., Gao, Y., Chen, C. J., and Zheng, J. (2011) Effects of heat treatments on microstructure and mechanical properties of $\mathrm{Mg}-11 \mathrm{Y}-5 \mathrm{Gd}-2 \mathrm{Zn}-0.5 \mathrm{Zr}$ (wt.\%) alloy J. Alloys Compd., 509, 1696-1704.

[228] Nie, J. F. (2003) Effects of precipitate shape and orientation on dispersion strengthening in magnesium alloys. Scr. Mater., 48, 1009-1015.

[229] Jain, J., Poole, W. J., Sinclair, C. W., and Gharghouri, M. A. (2010) Reducing the tension-compression yield asymmetry in a $\mathrm{Mg}-8 \mathrm{Al}-0.5 \mathrm{Zn}$ alloy via precipitation. Scr. Mater., 62, 301-304.

[230] Bohlen, J., Dobron, P., Swiostek, J., Letzig, D., Chmelik, F., Lukac, P., and Kainer, K. U. (2007) On the influence of the grain size and solute content on the AE response of magnesium alloys tested in tension and compression. Mater. Sci. Eng. A, 462, 302-306.

[231] Wang, Y. N. and Huang, J. C. (2007) The role of twinning and untwinning in yielding behavior in hot-extruded Mg-Al-Zn alloy. Acta Mater., 55, 897-905.

[232] Christ, H. -J. and Mughrabi, H. (1996) Cyclic stress-strain response and microstructure under variable amplitude loading. Fat. Frac. Eng. Mater. Struct., 19(2-3), 335-348.

[233] Li, Z. M., Wang, Q. G., Luo, A. A., Fu, P. H., Peng, L. M., Wang, Y. G., and Wu, G. H. (2013) High cycle fatigue of cast Mg-3Nd-0.2Zn magnesium alloys. Metall. Mater. Trans. $A, \mathbf{4 4 A}, 5202-5215$.

[234] Wen, L. H., Ji, Z. S., and Li, X. L. (2008) Effect of extrusion ratio on microstructure and mechanical properties of $\mathrm{Mg}-\mathrm{Nd}-\mathrm{Zn}-\mathrm{Zr}$ alloys prepared by a solid recycling process. Mater. Charact., 59, 1655-1660. 
[235] Ma, L., Peng, L. M., Jiang, H. Y., and Ding, W. J. (2012) Development of high strength and ductility Mg-Nd-Zn based wrought alloy. Mg2012: 9th International Conference on Magnesium Alloys and their Applications, Edited by W.J. Poole and K.U. Kainer, 9991005.

[236] Chaudhuri, B. and Das, P. P. Effect of different Variables on the Precipitate free Zones Adjacent to Grain Boundry Region in Conventionally Quenched and Aged AI-Zn-Mg Alloys. In: Symposium on Non-ferrous Metals Technology: National Metallurgical Laboratory (CSIR), Jamshedpur, 1968.

[237] Rateick, R. G., Griffith, R. J., Hall, D. A., and Thompson, K. A. (2005) Relationship of microstructure to fatigue strength loss in anodised aluminium-copper alloys. Mater. Sci. Technol., 21, 1227-1235.

[238] Zhang, C., Luo, A. A., Peng, L. M., Stone, D. S., and Chang, Y. A. (2011) Thermodynamic modeling and experimental investigation of the magnesium-neodymiumzinc alloys. Intermetallics, 19(11), 1720-1726.

[239] Fu, P. H., Peng, L. M., Jiang, H. Y., Ma, L., and Zhai, C. Q. (2008) Chemical composition optimization of gravity cast $\mathrm{Mg}-\mathrm{yNd}-\mathrm{XZn}-\mathrm{Zr}$ alloy. Mater. Sci. Eng. A, 496, 177-188.

[240] Fu, P. H., Peng, L. M., Jiang, H. Y., Chang, J. W., and Zhai, C. Q. (2008) Effects of heat treatments on the microstructures and mechanical properties of $\mathrm{Mg}-3 \mathrm{Nd}-0.2 \mathrm{Zn}-0.4 \mathrm{Zr}$ (wt.\%) alloy. Mater. Sci. Eng. A, 486, 183-192.

[241] Yu, K., Li, W. X., and Wang, R. C. (2005) Mechanical properties and microstructure of as-cast and extruded Mg-(Ce,Nd)-Zn-Zr alloys. J. Cent. South Univ. Technol., 12(5), 499502. 
[242] Zheng, F. Y., Wu, Y. J., Peng, L. M., Li, X. W., Fu, P. H., and Ding, W. J. (2013) Microstructures and mechanical properties of friction stir processed $\mathrm{Mg} 2.0 \mathrm{Nd} 0.3 \mathrm{Zn} 1.0 \mathrm{Zr}$ magnesium alloy. J. Magnesium alloys, 1(2), 122-127.

[243] Ma, L., Mishra, R. K., Peng, L. M., Luo, A. A., Ding, W. J., and Sachdev, A. K. (2011) Texture and mechanical behavior evolution of age-hardenable $\mathrm{Mg}-\mathrm{Nd}-\mathrm{Zn}$ extrusions during aging treatment. Mater. Sci. Eng. A, 529, 151-155.

[244] Dong, J., Zheng, X. W., Zhang, Z. Y., Wang, Y. X., Jin, L., and Ding, W. J. (2011) Plastic formability and auto demonstration parts of $\mathrm{Mg}-\mathrm{Nd}-\mathrm{Zn}-\mathrm{Zr}$ magnesium alloy. Mater. Sci. Forum, 690, 455-458.

[245] Li, Z. M., Luo, A. A., Wang, Q. G., Peng, L. M., Fu, P. H., and Wu, G. H. (2013) Effects of grain size and heat treatment on the tensile properties of $\mathrm{Mg}-3 \mathrm{Nd}-0.2 \mathrm{Zn}$ (wt.\%) magnesium alloys. Mater. Sci. Eng. A, 564, 450-460.

[246] Yoo, M. H., Morris, J. R., Ho, K. M., and Agnew, S. R. (2001) Non-basal slip systems in HCP metals and alloys: Source mechanisms. Mater. Sci. Eng. A, A319-A321, pp. 87-92.

[247] Liu, B. Y., Wang, J., Li, B., Lu, L., Zhang, X. Y., Shan, Z. W., Li J., Jia, C. L., Sun, J., and Ma, E. (2014) Twinning-like lattice reorientation without a crystallographic twinning plane. Nature Comm, 5, Article number 3297.

[248] Xin, Y. C., Wang, M. Y., Zeng, Z., Nie, M. G., and Liu, Q. (2012) Strengthening and toughening of magnesium alloy by $\{10-12\}$ extension twins. Scr. Mater.,66, 25-28. 